\title{
Research on Carbon Products from Coal \\ Using an Extractive Process
}

Final Report

Report Period Start Date: $\quad$ September 27, 2000

Report Period End Date: $\quad$ December 31, 2001

Principal Authors: $\quad$ Peter G. Stansberry

Alfred H. Stiller

John W. Zondlo

Chong Chen

Brian Bland

David Fenton

Report Issued: $\quad$ March 31, 2002

DOE Award Number: $\quad$ DE-FC26-00NT41008

Submitting Organization: Department of Chemical Engineering

West Virginia University

PO Box 6102

Morgantown, WV 26506-6102

USA 


\section{DISCLAIMER}

"This report was prepared as an account of work sponsored by an agency of the United State Government. Neither the United State Government nor any agency thereof, nor any of their employees, makes any warranty, express or implied, or assumes any real liability or responsibility for the accuracy, completeness, or usefulness of any information, apparatus, product, or process disclosed, or represents that its use would not infringe privately owned rights. Reference herein to any specific commercial product, process, or service by trade name, trademark, manufacturer, or otherwise does not necessarily constitute or imply its endorsement, recommendation, or favoring by the United States Government or any agency thereof. The view and opinions of authors expressed herein do not necessarily state or reflect those of the United States Government or any agency thereof." 


\section{1. $\underline{\text { Abstract }}$}

This report presents the results of a one-year effort directed at the exploration of the use of coal as a feedstock for a variety of industrially-relevant carbon products. The work was basically divided into three focus areas. The first area dealt with the acquisition of laboratory equipment to aid in the analysis and characterization of both the raw coal and the coal-derived feedstocks. Improvements were also made on the coal-extraction pilot plant which will now allow larger quantities of feedstock to be produced. Mass and energy balances were also performed on the pilot plant in an attempt to evaluate the scale-up potential of the process.

The second focus area dealt with exploring hydrogenation conditions specifically aimed at testing several less-expensive candidate hydrogen-donor solvents. Through a process of filtration and vacuum distillation, viable pitch products were produced and evaluated. Moreover, a recycle solvent was also isolated so that the overall solvent balance in the system could be maintained. The effect of variables such as gas pressure and gas atmosphere were evaluated. The pitch product was analyzed and showed low ash content, reasonable yield, good coking value and a coke with anisotropic optical texture. A unique plot of coke yield vs. pitch softening point was discovered to be independent of reaction conditions or hydrogen-donor solvent.

The third area of research centered on the investigation of alternate extraction solvents and processing conditions for the solvent extraction step. A wide variety of solvents, co-solvents and enhancement additives were tested with varying degrees of success. For the extraction of raw coal, the efficacy of the alternate solvents when compared to the benchmark solvent, N-methyl pyrrolidone, was not good. However when the same coal was partially hydrogenated prior to solvent extraction, all solvents showed excellent results even for extractions performed at room temperature. Standard analyses of the extraction products indicated that they had the requisite properties of viable carbon-product precursors. 


\section{Table of Contents}

1. Abstract

2. Table of Contents

3. List of Figures

4. List of Tables

5. Introduction

6. Task 1.0 Facilities and Improvements and Installation and Pilot Scale Testing $1.1 \quad$ Introduction 1.1.1 WVU NMP Extraction History

1.2 Background

1.2.1 Upper Powelton (WVGS13421)

1.2.2 What is Solvent Extraction of Coal?

1.2.3 Coal and Solvent Properties Effecting Extraction

1.2.4 Coal Properties Effecting Extraction

1.2.5 Solvent Nature and Physical Properties Effecting Extraction

1.2.6 Supersolvents

1.2.7 Studies of Supersolvents on Coal

1.2.8 N-methyl-2-pyrrolidone (NMP, The Solvent of Choice

1.2.9 Parametric Study of Solvent Extraction

1.2.9.1 Effect of Time and Temperature (Kinetics of Solvent Extraction)

1.2.9.2 Effect of Particle Size on Extraction Yield

1.2.9.3 Effect of Oxygen and Moisture on Extraction

1.2.9.4 Solvent Swelling of Coal

1.2.10 What is the Product of Solvent Extraction of Coal?

1.3 Experimental

1.3.1 Comparison of Laboratory Procedure and Pilot Scale Operation

1.3.1.1 Laboratory Scale Pitch Production

1.3.1.2 Pitch Production in the coal Extraction Pilot Unit (CEPU)

1.3.2 Construction of the Coal Extraction Pilot Unit

1.3.3 Pilot Plant Construction Timeline

1.3.4 CEPU Equipment Design

1.3.5 Safety Concerns and Chemical Hygiene Plan

1.3.6 Mezzanine Preparations and Component Manufacturing

1.4 CEPU Startup

1.4.1 First and Second Run with NMP

1.4.2 First Run with NMP and coal

1.4.3 TFE Startup

1.4.4 Drying Ovens and Rotovap First Runs

1.4.5 Continuously Stirred Tank Reactor (R-101)

1.4.6 Centrifuge (Ct-101) 
1.4.7 Wiped Thin Film Evaporator (TFE-101)

1.4.8 Rotovap (RV-101)

1.4.9 Drying Ovens (DO-101-3)

1.5 Results

1.5.1 Material Balance

1.5.2 Overall Material Balance

1.5.3 Material Balance Over Individual Units

1.5.3.1 Material Balance Over Reactor (CSTR) (R-101)

1.5.3.2 Material Balance Over Centrifuge (Ct-101)

1.5.3.3 Material Balance Over TFE (TFE-101)

1.5.3.4 Material Balance Over Rotary Evaporator (RV-101)

1.5.3.5 Material Balance Over Drying Ovens (DO-101-3)

1.6 Energy Balance

1.6.1 Thermal Properties of NMP

1.6.2 Thermal Properties of Coal

1.6.3 Energy Balance on Reactor (CSTR) (R-101)

1.6.4 Reactor $\mathrm{NMP}_{2} \mathrm{O}$ Vapor Condenser (HE-101)

1.6.5 Reactor Slurry Cooler Before Centrifuge (HE-104)

1.6.6 Energy Balance on Wiped Thin Film Evaporator (TFE-101)

1.6.7 Energy Balance on Rotary Evaporator (Rotovap) (RV-101)

1.6.8 Energy Balance on Drying Ovens (DO-101-3)

1.7 Conclusions

1.8 Alterations to the Pilot Plant

1.9 References

7. Task 2.0 Investigation of Hydrogenation Conditions

2.1 Introduction

2.2 Experimental Procedure for Hydrogenation Reactions

2.2.1 Overview of Hydrogenation Reaction

2.2.2 Fluidized Sand Bath Preparation

2.2.3 Reactor Preparation

2.2.4 Reactor Charging

2.2.5 Gas Charging

2.2.6 Reaction Procedure

2.2.7 Product Collection

2.2.8 THF Extraction

2.2.9 Product Isolation

2.3 Coal Extract Testing

2.3.1 Softening Point

2.3.2 Coking Value

2.3.3 Ash Content

2.3.4 Optical Texture

2.3.5 Elemental Analysis

2.3.6 Error Calculation

2.4 Results and Discussion

2.4.1 Mass Balances 
2.4.2 Ash Balance

2.4.3 Solvent Evaluation

2.4.4 Reaction Pressure

2.4.5 Product Distillation

2.4.6 Hydrogenation Products

2.4.6.1 Ash Content

2.4.6.2 Coke Yield

2.4.6.3 Optical Texture

2.4.6.4 Elemental Analysis

2.4.7 Variation of Hydrogenation Parameters

2.4.7.1 Effect of Pressure and Atmosphere

2.4.7.2 Effect of Solvent-to-Coal Ratio

2.5 Conclusions

2.6 References

8. Task 3.0 Laboratory Scale Testing of Alternate solvents

3.1 Introduction

3.2 Experimental

3.2.1 Coal Samples

3.2.2 Solvents and Chemicals

3.2.3 Solvent Extraction

3.2.4 Hydrogenation of Coal

3.2.5 Thermal Dissolution of Coal

3.2.5.1 NMP

3.2.5.2 Creosote Oil or Coal-Derived Pitch

3.2.6 Swelling Kinetics of Coal

3.2.7 Characterization of Coal and Coal Extracts

3.3 Results and Discussion

3.3.1 Extraction of Raw Coal

3.3.2 Extraction of Hydrogenated Coal

3.3.3 Synergistic Effect of $\mathrm{CS}_{2}$

3.3.4 Effect of Ultrasonic Radiation on Extraction

3.3.5 Effect of Additives

3.3.5.1 Effect of Additives on the Extraction Behavior

3.3.5.2 Effect of Additives on Pitch Properties

3.3.6 Thermal Dissolution of Coal

3.3.7 Swelling Kinetics of Coal in Solvents

3.4 Conclusions

3.5 References 


\section{List of Figures}

Figure 1.1 Block flow diagram for laboratory production of coal-based carbon pitch.

Figure 1.2 Block flow diagram for pilot plant production of coal-based carbon pitch

Figure 1.3 CEPU construction time task deliverable chart.

Figure 1.4 Process flow diagram for production of coal-based carbon pitch.

Figure 1.5 Process flow diagram for production of coal-based carbon pitch

Figure 1.6 Continuous stirred tank reactor with heat exchanger, pump, condenser, and absorber.

Figure 1.7 CSTR lid showing motor, cap, and condenser.

Figure 1.8 Sharples Penwalt BM-PF 290 centrifuge.

Figure 1.9 Pfaudler $1.2 \mathrm{ft}^{2}$ wiped thin film evaporator.

Figure 1.10 Thickened extract leaving wiped thin film evaporator.

Figure 1.11 Modified Buchi R-135 rotary evaporator with steel tank.

Figure 1.12 Processed coal and pitch drying ovens and heat exchanger.

Figure 1.13 Drying oven condenser (HE-105) and collection tank (T-106).

Figure 1.14 Block flow diagram with stream compositions for production of coal-based carbon pitch.

Figure 1.15 Typical values of the specific heats of coal, coke, and graphite (Bolborth 1987).

Figure 1.16 CSTR (R-101) temperature profile for heating.

Figure 1.17 CSTR (R-101) temperature profile for cooling the reactor without supporting heat exchanger HE-104.

Figure 1.18 CSTR (R-101) slurry cooling before centrifuge (CT-101) with supporting heat exchanger (HE-104).

Figure 1.19 Log of NMP saturation pressure vs. temperature

Figure 1.20 Charles Ross \& Son (HDM 40) at test site in NY. 
Figure 1.21 HDM 40 with condenser at New York.

Figure 1.22 Double planetary mixing blades with wall scraper

Figure 1.23 Double planetary mixing blades with product

Figure 1.24 Ross installed on CEPU

Figure 2.1 Experimental procedure flow sheet for production of carbon product precursors for coal hydrogenation reactions.

Figure 2.2 Diagramof tubing bomb microreactor (TBMR)

Figure 2.3 The effect of the hydrogenation solvent on the overall conversion to THF solubles at the reaction conditions of $400{ }^{\circ} \mathrm{C}, 1000 \mathrm{psig}_{2}$ (cold), 3/1 solvent-to-coal ratio, and 1 hour.

Figure 2.4 The effect of the hydrogenation solvent on the coal-alone conversion (daf basis) of coal to THF solubles at the reaction conditions of $400{ }^{\circ} \mathrm{C}, 1000 \mathrm{psig}_{2}$ (cold), 3/1 solvent-to-coal ratio, and 1 hour.

Figure 2.5 Reactor pressures during coal hydrogenation reactions with coal-derived solvents at reaction conditions of $400{ }^{\circ} \mathrm{C}, 1000 \mathrm{psig}_{2}$ (cold), 3/1 solvent-to-coal ratio, and 1 hour.

Figure 2.6 Effect of distillation on the softening point of the pitch resulting from hydrogenation reactions with coal-derived solvents at reaction conditions of $400{ }^{\circ} \mathrm{C}, 1000 \mathrm{psig}_{2}$ (cold), 3/1 solvent-to-coal ratio, and 1 hour.

Figure 2.7 Effect of solvent choice on the hydrogenation product distribution with reaction conditions of $400{ }^{\circ} \mathrm{C}, 1000 \mathrm{psig}_{2}$ (cold), 3/1 solvent-to-coal ratio, and 1 hour for a pitch with approximately $120^{\circ} \mathrm{C}$ softening point.

Figure 2.8 Effect of hydrogenation solvent on the coke yield of the pitches resulting from hydrogenation reactions with the conditions of $400{ }^{\circ} \mathrm{C}, 1000 \mathrm{psig}_{2}$ (cold), 3/1 solvent-to-coal ratio, and 1 hour. Pitch softening point is around $120^{\circ} \mathrm{C}$.

Figure 2.9 Effect of softening point on the coke yield of pitches made from hydrogenation reactions using the five different coal-derived solvents under reaction conditions of $400{ }^{\circ} \mathrm{C}, 1000 \mathrm{psig}_{2}$ (cold), 3/1 solvent-to-coal ratio, and 1 hour.

Figure 2.10 Optical micrographs of cokes produced from pitches (with softening points around $120^{\circ} \mathrm{C}$ ) using different solvents during hydrogenation reactions with conditions of 
$400{ }^{\circ} \mathrm{C}, 1000 \mathrm{psig} \mathrm{H}_{2}$ (cold), $3 / 1$ solvent-to-coal ratio, and 1 hour and solvents a) DACL-L, B) DACL-H, C) RACL, D) HCO, and E) CBB.

Figure 2.11 Effect of initial reactor pressure and gaseous atmosphere on the overall conversion of hydrogenation reactions with $\mathrm{CBB}$ and reaction conditions of $400{ }^{\circ} \mathrm{C}, 3 / 1$ solventto-coal ratio, and 1 hour.

Figure 2.12 Effect of initial reactor pressure and gaseous atmosphere on the coal-alone conversion of hydrogenation reactions with $\mathrm{CBB}$ and reaction conditions of $400{ }^{\circ} \mathrm{C}, 3 / 1$ solventto-coal ratio, and 1 hour.

Figure 2.13 Average reactor pressures over time of hydrogenation reactions using CBB, 1000 psig initial hydrogen or argon pressure, $400{ }^{\circ} \mathrm{C}, 3 / 1$ solvent-to-coal ratio, and 1 hour.

Figure 2.14 Average reactor pressures over time of hydrogenation reactions using CBB, 500 psig initial hydrogen or argon pressure, $400^{\circ} \mathrm{C}, 3 / 1$ solvent-to-coal ratio, and 1 hour.

Figure 2.15 Average reactor pressures over time of hydrogenation reactions using CBB, 100 psig initial hydrogen or argon pressure, $400^{\circ} \mathrm{C}, 3 / 1$ solvent-to-coal ratio, and 1 hour.

Figure 2.16 Effect of initial reactor pressure and atmosphere on the product distribution of reactions run to produce a pitch with a softening point of $120^{\circ} \mathrm{C}$ under reaction conditions of $\mathrm{CBB}$ solvent, $400^{\circ} \mathrm{C}, 3 / 1$ solvent-to-coal ratio, and 1 hour.

Figure 2.17 Effect of raising the softening point of pitches produced under different atmospheres and initial reaction pressures on the coke yield with reaction conditions of CBB solvent, $400^{\circ} \mathrm{C}, 3 / 1$ solvent-to-coal ratio, and 1 hour.

Figure 2.18 Optical micrographs of cokes produced from select pitches derived from hydrogenation products from reactions $\left(\mathrm{CBB}\right.$ solvent, $400^{\circ} \mathrm{C}, 3 / 1$ solvent-to-coal ratio, 1 hour) with varying initial pressures and atmosphers, A) 1000 psig Hydrogen, B) 1000 psig Argon, C) 500 psig Hydrogen, D) 500 psig Argon, E) 100 psig Hydrogen, and F) 100 psig Argon.

Figure 2.19 Effect of solvent-to-coal ratio on the overall and coal-alone conversions of hydrogenation reactions performed with the $\mathrm{HCO}$ solvent under conditions of $400^{\circ} \mathrm{C}$, 1000 psig Hydrogen, and 1 hour.

Figure 2.20 Effect of solvent-to-coal ratio on the amount of distillation required to achieve a specific softening point from hydrogenation reactions with conditions of $\mathrm{HCO}$ solvent, $400^{\circ} \mathrm{C}, 1000$ psig Hydrogen, and 1 hour.

Figure 2.21 Effect of lowering the solvent-to-coal ratio on the pitch coke yield and various softening points from hydrogenation reactions with conditions of $\mathrm{HCO}$ solvent, $400^{\circ} \mathrm{C}, 1000$ psig Hydrogen, and 1 hour. 
Figure 2.22 Effect of pitch softening point on the coke yield for all pitch fractions produced under varying reaction conditions.

Figure 3.1 Change of coal conversion yield to NMP solubles as the heat treatment temperature increase. Heat treatment medium/extractant: NMP. Heat treatment/extraction crried out at same time for 1 hour with agitation. Coal: WVCS001.

Figure 3.2 FTIR spectra of NMP solubles from WVSC001 coal heat treated at $400^{\circ} \mathrm{C}$ in NMP. $\mathrm{a}$ - solubles collected by water precipitation; $\mathrm{b}$ - solubles collected by vacuum evaporation.

Figure 3.3 Swelling curve of WVSC-001 in DMF, top; and the kinetic curve from equation 3.1, bottom.

Figure 3.4 Swelling curve of WVSC-001 in DMAC, top; and the kinetic curve from equation 3.1 , bottom.

Figure 3.5 Swelling curve of WVSC-001 in NMP, top; and the kinetic curve from equation 3.1, bottom. 


\section{List of Tables}

Table 1.1 Key Physical and Chemical Properties of N-methyl-2-pyrrolidone.

Table 1.2 Potential Uses of Coal Extracts

Table 1.3 Coal Extraction Pilot Unit Stream Table.

Table 1.4 Equipment List for coal Extraction Pilot Unit

Table 1.5 Material Balance Results

Table 1.6 TFE Normal Operating Parameters

Table 2.1 Characteristics of WVGS 13421.

Table 2.2 Elemental Compositions of WVGS 13421 and Coal-Derived Solvents.

Table 2.3 Hydrogenation Reaction Conditions.

Table 2.4 Overall Mass Balances of the Coal Hydrogenation Reactions.

Table 2.5 Ash Balance of Hydrogenation Reactants and Products.

Table 2.6 Softening Point of Pitch and Ash Content of Pitch and THF Insoluble Fractions of Hydrogenation Products.

Table 2.7 Elemental Composition of Select Hydrogenation Products.

Table 2.8 Elemental Carbon Balance for Hydrogenation Reactants and Products.

Table 2.9 Elemental Hydrogen Balance for Hydrogenation Reactants and Product.

Table 2.10 Ash Content of Pitch and THF Insoluble Fractions of Pitch Produced While Varying the Initial Reactor Pressure and Gaseous Atmosphere.

Table 2.11 Elemental Composition of Select Pitches Produced from Hydrogenation Reactions (CBB Solvent, $400^{\circ} \mathrm{C}, 3 / 1$ solvent-to-coal ratio, 1 hour) with different initial pressure and atmospheres: 5B) 1000 psig Hydrogen, 6D) 1000 psig Argon, 7A) 500 psig Hydrogen, 8D) 500 psig Argon, 9B) 100 psig Hydrogen, and 10D) 100 psig Argon.

Table 2.12 Carbon Balance of Select Pitches Produced From Hydrogenation Reactions with 
Differential Initial Pressures and Atmospheres: 5B) 1000 psig Hydrogen, 6D) 1000 psig Argon, 7A) 500 psig Hydrogen, 8D) 500 psig Argon, 9B) 100 psig Hydrogen, and 10D) 100 psig Argon.

Table 2.13 Hydrogen Balance of Select Pitches Produced from Hydrogenation Reactions with Different Initial Pressure and Atmospheres: 5B) 1000 psig Hydrogen, 6D) 1000 psig Argon, 7A) 500 psig Hydrogen, 8D) 500 psig Argon, 9B) 100 psig Hydrogen, and 10D) 100 psig Argon.

Table 2.14 Ash content of Pitch and THF Insoluble Fractions of Selected Hydogenation Runs with Decreasing Solvent-to-Coal Ratio with Reaction Conditions of HCO Solvent, $400^{\circ} \mathrm{C}, 1000$ psig Hydrogen, and 1 Hour.

Table 2.15 Elemental Analysis of Selected Hydrogenation Trials as the Solvent-to-Coal Ratio was Lowered but all Other Reaction Conditions were kept Constant at HCO Solvent, $400^{\circ} \mathrm{C}, 1000$ psig Hydrogn, and 1 Hour.

Table 3.1 Proximate and Ultimate Analysis of Coals.

Table 3.2 Extraction Yield of Raw Coal Using Various Solvents.

Table 3.3 Effect of Hydrogenation on the Solubility of Coal (WVGS13421) in the Solvents. Extraction is at the Boiling Point of the Solvent for 1 Hour.

Table 3.4 Comparison of the Extraction Yield of Hydrogenated Coal Using Different Solvents.

Table 3.5 Elemental Analysis of Raw Coal, Hydrogenated Coal and Their NMP Extracts.

Table 3.6 Extraction Yield of NMP/CS 2 Mixed Solvent for Selected West Virginia and Pennsylvania Bituminous Coal. Extraction Carried out at Room Temperature.

Table 3.7 Comparison of the Extraction Yield Using Pure Solvent and $\mathrm{CS}_{2}$ Mixed Solvent Extraction Carried Out at Room Temperature for 1 Hour.

Table 3.8 Comparison of the Effect of Ultrasonic Radiation and Magnetic Stirring on the Extraction Yield. Coal, WVGS13421. Extraction at Room Temperature for 1 Hour.

Table 3.9 Effect of Additives on the Extraction Yield (wt\%) of WVGS13421 Coal in Various Solvents (Extraction at the Boiling Point of the Solvent).

Table 3.10 Elemental Analysis of Coal and its Extracts and Residues from NMP Extraction.

Table 3.11 Effect of Additive on the Solvent Extraction Yield of Hydrogenated Coal (WVGS13421). Extraction Carried Out at Room Temperature.

Table 3.12 Effect of Some Additives on Coal Tar Pitch's Properties. 
Table 3.13 Coal Conversion Yield of Thermal Dissolution of Coal, wt $\%$.

Table 3.14 Comparison of the Elemental Composition of Raw Coal and the Corresponding NMP Solubles. [Thermal Dissolution at $400^{\circ} \mathrm{C}$ Using NMP as Heat Treatment Medium and Extraction Solvent.]

Table 3.15 Swelling Ratio of Coal in Solvents at Room Temperature. 


\section{Introduction}

Currently, the primary source of carbon materials is as a by-product of the petroleum refinery or the metallurgical coke oven. Strategic, technical and environmental constraints make it imperative that a new source of this feedstock be obtained. Coal, though attractive in terms of domestic availability and supply, is disadvantageous in that it contains ash and other environmentally sensitive components such as sulfur. However, engineers and scientists of the Carbon Products Program in the Department of Chemical Engineering at West Virginia University (WVU) have developed a coal pitch extract which is virtually free of ash and other inorganic material; this extract can be used as a precursor for a variety of carbon-based materials. The extract is obtained by using a class of organic solvents known generically as dipolar aprotic compounds. The solvent extraction (of "raw" coal or hydrogenated coal) is performed at relatively mild conditions of approximately $200 \mathrm{C}$ and atmospheric pressure. The undissolved solid material is separated by simple filtration and the dissolved organic material is recovered by evaporating the solvent. This leaves a friable, glass-like solid. By blending the extract from raw coal and the extract from hydrogenated coal, and/or by changing the conditions of hydrogenation of the coal prior to extraction, pitches of various properties have been created. Materials which have been made using these pitches as a starting point include coke, suitable for anodes in the aluminum industry; graphite, potentially suitable for steel processing and use in the nuclear industry; binder pitch; mesophase pitch; and carbon foam. Other possible novel products under development include carbon beads, wool, fibers, nanotubes and carbon-carbon composites.

The above-described process has formed the cornerstone of the fundamental research for the WVU program on coal-derived carbon materials. The program has successfully demonstrated that many of the industrially-relevant carbon products currently made from petroleum feedstocks can be produced from coal. However, to be of use to the State of West Virginia and the carbon industry in general, progress must be made toward commercialization of one or more of these processes. To this end, a small pilot plant has been constructed at WVU, capable of producing approximately ten pounds of material per run. Further, significant industrial contacts have been established, both through membership in two consortia as well as interaction with groups intent on commercializing the technology. Moreover, three patents have already been issued as an outgrowth of this program and several more are pending. Currently, two are under negotiation for licensing.

One important aspect of the success of this program lies in the establishment of the process economics and ultimate product cost. To this end, much of the work presented in this report is focused on improving the process to yield more attractive economics and to gather pertinent data necessary for a cogent economic analysis.

Hence, the ultimate vision of the program is two-pronged. First, emphasis will be placed on process development and improvement in order to enhance the economics. Secondly, product focus areas will be targeted such that new and exciting applications of the coal as feedstock can be attained.

The results of the work presented here are focused on three key areas of research: 
Work concentrated on both the installation of laboratory/analytical equipment as well as improvement of the pilot plant facility. For the latter, new equipment was added and tested for more efficient drying of the extract which will lead to improved recovery of the solvent. In the laboratory, a new elemental $(\mathrm{C}, \mathrm{H}, \mathrm{N}, \mathrm{S}, \mathrm{O})$ analyzer was purchased to aid in analysis of coal and coal-based carbon precursors. In addition, a 5-gallon autoclave reactor was purchased so that larger quantities of hydrogenated coal could be produced for testing and evaluation of scale-up. Moreover, the processing steps were carefully assessed in the pilot plant. This allowed a more thorough understanding of each unit operation as well as the acquisition of valuable scale-up data such as mass and energy balances. In the following report, these aspects (originally labeled Task 1 and 4 in the proposal) will be covered under a single section - Task I.

\section{B) Investigation of Hydrogenation Conditions:}

Hydrogenation of the raw coal is one of the key steps in converting coal to viable carbon products. By adjusting the hydrogenation conditions, the molecular weight and chemical character of the resulting product can be controlled. This in turn dictates the properties and uses of the carbon products made from the hydrogenated coal. In this task, Task 2, a wide range of hydrogenations were performed in a variety of different H-donor solvents. The effort focused on the nature of the solvent, the conversion yield of the coal, the properties of the resultant coal-derived pitch and the recovery of the solvent for recycle. Various properties of the pitch were tested and included ash concentration, coke yield, elemental analysis and the optical texture of the cokes. Serious emphasis was placed on solvent recovery and recycle such that a sustained process could be realized. The results of this work are reported under Task 2 which follows.

\section{C) Laboratory-Scale Testing of Alternative Solvents:}

Two of the primary considerations of the economics of the process are with cost of the extraction solvent and the yield of coal extract product. Typically the solvent N-methyl pyrrolidone (NMP) has been used by the WVU group in the past. However, this solvent is costly. Hence the search for some cheaper solvents was undertaken. Due to close industrial ties, two alternate solvent, dimethyl formamide (DMF) and dimethyl acetamide (DMAC) were tested. In addition a variety of co-solvents and enhancement additives were evaluated. All tests were performed on both raw and hydrogenated coal. The resultant extracts were characterized by many techniques, namely: softening point temperature, elemental analysis, ash concentration, coke yield, swelling and optical texture of the coke. The objective here was to reduce the overall process costs by a combination of less-severe operating conditions, cheaper solvents, improved yields and good product characteristics. The results of this work are presented under Task 3 which follows.

For the sake of continuity, each of the three tasks will be presented separately as a complete unit. Hence the experiments, results and discussion, and conclusions will be specific to the particular task under discussion. 


\section{Task 1.0 Facilities and Improvements and Installation and Pilot Scale Testing}

\subsection{Introduction}

\subsubsection{WVU NMP Extraction History}

For the past several years, the Department of Chemical Engineering at West Virginia University (WVU) College of Engineering and Mineral Resources (CEMR) has been conducting research into the use of coal as a raw material for producing feedstocks for carbon products. The focus of these studies has been the dissolution of upper Powelton and other bituminous coals in an aprotic dipolar solvent. This research has led to the development of a solvent extraction technique that uses N-methyl-2-pyrrolidone (NMP) to dissolve large fractions of organic materials from coal. The mineral matter (referred to as ash) and inorganic sulfur remain undissolved in the solvent. This solvent has been shown to dissolve over $50 \%$ of the total carbonaceous carbon material in coal. In sequential steps the insoluble materials including the ash and some organic material are separated from the soluble materials by using a centrifuge or pressure filter. The filtrate, that contains all the soluble coal materials, is evaporated to dryness and a dry "solvent free" solid, carbonaceous material that contains less than 1 percent ash and no detectable pyritic or inorganic sulfur results (Gerstner [1989]). This solid, referred to as "reconstituted coal", coal based carbon pitch, extract, or just pitch has unique properties.

1) The heating value has increased to over $14,500 \mathrm{BTU} / \mathrm{lb}$, as compared to the original Powelton coal, which averages at $12,500 \mathrm{BTU} / \mathrm{lb}$. This increase in heating value is because the ash, which has little BTU value, has been removed.

2) The pitch produces an ash-free isotropic coke. When graphitized the isotopic coke produces graphite that has very small randomly orientated crystallite domains

3) The carbon yield (amount of fixed carbon after coking) of the reconstituted coal is about $63 \%$, which is fairly high.

This newly-developed coal extraction technique of de-ashing and extraction of coal using $\mathrm{N}$-methyl-2-pyrrolidone (NMP) at atmospheric pressure and moderate temperature $\left(202^{\circ} \mathrm{C}\right)$ has been carefully studied at WVU by the Chemical Engineering Department but only at the bench scale. These studies were done by K. Renganathan (1986), G. R. Phillips (1988), J. A. Gerstner (1989), S. D. Gadam (1990), V. P. Bonifera Jr. (1997), and W.R. Stewart (1992). Cost evaluations of possible industrial scale systems of any kind were empirically developed to estimate cost per ton of this product.

One of the goals of this research explores the scale-up of production by constructing a pilot plant that will permit more complete material and energy balances. Until now, no pilot plant existed to address the issue of actual production of any appreciable quantity of the coalbased carbon extract. Limited data existed to upscale the process toward industrial scale production. For the most part, the issues of chemical safety, quantity, solvent recovery, 
quality of the product, and material handling and processing had not been satisfactorily addressed. The majority of the data collection on the extraction technique of coal based carbon product was preformed in a laboratory using closely controlled conditions and is inappropriate for industrial scale up. The primary goal of this research is to develop an actual flow sheet, and complete design and construction for a pilot plant at WVU to collect appropriate data for scale up.

This report discusses the design, construction, operation, and preliminary evaluations of a pilot scale operation for a coal based Carbon Extraction Pilot Unit (CEPU). The specific objectives for this research include:

1. Design and construction of the CEPU;

2. Perform a material balance on the CEPU

3. Perform an energy balance on the CEPU

4. Implement process improvements for production.

\subsection{Background}

\subsubsection{Upper Powelton Coal (West Virginia Geological Survey \# 13421)}

Upper Powelton (WVGS 13421) coal was chosen for this research based on its availability and solubility in NMP. Leckie Smokless Coal Co. donated all the coal for this research to West Virginia University. Data from lab scale tests show the average dissolution for this coal in NMP has a yield of $38 \%$.

\subsubsection{What is Solvent Extraction of Coal?}

Solvent extraction is the method of using a solvent to dissolve a desired portion of coal and extract or separate it from the undesired portion of coal. This can be explained by comparing it to the separation of sugar and rice with water. Imagine that the coal is actually made of a solid mixture of sugar and rice. If this mixture is placed into water, the sugar will dissolve, but the rice will remain unaffected. You can then separate the sugar that dissolved from the rice that didn't by simple filtration. In a similar manner a certain portion of the coal will dissolve if placed in the proper solvent. The dissolved portion of the coal can be separated from the undissolved portion by filtration or centrifugation. The water can be driven off and the dry sugar recovered in a manner similar to driving off the solvent and recovering the dissolved portion of coal.

The solvent extraction of coal has been employed for many years as a means of studying the constitution of coal and as a coal upgrading technique. Unlike many other coal upgrading processes, solvent extraction utilizes relatively less severe treatment conditions and leaves the greater part of its products similar to the original coal. Attempts have been made in the past to explain various coal properties, coking behavior, rank of coal, degree of oxidation, and so forth, on the basis of solvent action on coals (Gadam 1990). 


\subsubsection{Coal and Solvent Properties Effecting Extraction}

Several attempts have been made to correlate coal solubility with some physical parameter of either the solvent and/or the coal. Illingworth (1922) observed that when certain coals were heated to 200 to $400^{\circ} \mathrm{C}$ in an inert atmosphere, cooled subsequently and extracted with solvents, the yield was higher than that from untreated coals. Asbury (1934) studied the influence of time on extraction. Asbury found that after the initial extraction period of 32 hours, the relation between time and time-yield was linear and its slope gave the ultimate yield. Keppler and Brochers (1934) found that an increase in temperature increased the extent of extraction. Curless (1963) showed that a smaller size of coal particle produced a higher yield of extraction.

Dryden $(1950,1951)$ reviewed the voluminous literature on solvent extraction of coal. Compiling this review with his own research (1951), Dryden concluded that the coal solubility in the solvent had very little correlation with the internal pressure, surface tension, dielectric constant, or dipole moment of solvent. He distinguished solvents capable of breaking the intermolecular bonds in coal from those that can stabilize coal fragments in solution. Dryden proposed that a family of solvents containing a nitrogen or oxygen atom possessing an unshared pair of electrons were desirable solvents.

Dryden proposed a model in 1955 to explain the process of dissolution based on the assumption that coal consisted of units called micelles. These micelles are a smaller unit of the macerals, which can vary in size over a range of 1 to 300 microns and are assumed to be indestructible. The desired family of solvents mentioned above act by swelling the micellar network so that the smaller units of coal could be extracted out of the swelled structure. Dryden substantiated this micellar-sieve extraction mechanism by showing that the extent of extraction was proportional to the extent of swelling. He then stated that a solvent was suitable if it promoted swelling of the coal structure while simultaneously stabilizing the coal micelles in solution. The swelling promotes loosening. The availability of an unshared pair of electrons was considered to be an important factor (Renganathan 1986).

Dryden (1951) has shown that physical properties like surface tension, dielectric constant and dipole moment are of little assistance in classifying solvents for coal extractions. Moreover, the approach of relating physical properties of the solvent to the dissolvability of the solvent is of little value in scientific understanding of the extraction process (Gadam 1990).

\subsubsection{Coal Properties Effecting Extraction}

The dissolvability of coal decreases rapidly as the carbon composition of coal increases from 85 to 89 -weight \% (dry ash free basis). The dissolvability then becomes almost negligible at 92 to $93 \% \mathrm{wt} \%$ carbon. This statement applies broadly to all temperatures and types of solvents. Of the petrological constituents, vitrain yields the most extract and fusain the least (Gadam 1990). 


\subsubsection{Solvent Nature and Physical Properties Effecting Extraction}

Years of research have been devoted to determining which solvent dissolves what rank, how much, and what proportions of coals. Kiebler put forth a detailed discussion of the effect of the solvent nature (Lowry, 1945). Kiebler's results and can be summed up as follows: "Aromatic solvents are more effective than neutral aliphatic solvents". Extractions with benzene and similar solvents do not peptize the coal, while pyridine extractions do. The acidic or basic nature of aromatic solvents is unimportant although it may affect the yield owing to its contribution to internal pressure (Gadam 1990).

Different solvents can have different types of interactions with the same coal resulting from the specific chemical structure of the solvent. The high solvent power of amines and related solvents for lower rank bituminous coals can be accredited to the presence of an unshared pair of electrons on the nitrogen atom (Gadam 1990). Evidence has been obtained showing that the solvent power is closely related to the presence of hydroxyl groups. A two-ring system containing a hydroxyl group attached to an aromatic ring and a hydroaromatic ring was found to be very effective (Dryden, 1950).

The point of whether the solvent action on coal is physical or chemical in nature has always been disputed. It is suspected that certain organic liquids are performing more than a solvent action on coal. An indication of chemical interaction is obtained from the observation that the total weight of the products sometimes exceeds that of the original coal (Lowry, 1945).

\subsubsection{Supersolvents}

The term "supersolvents" is used to denote dipolar aprotic solvents that are capable of dissolving a large fraction of organic material in coal (Stiller et al., 1981). As Stiller states "Supersolvents have the general formula,

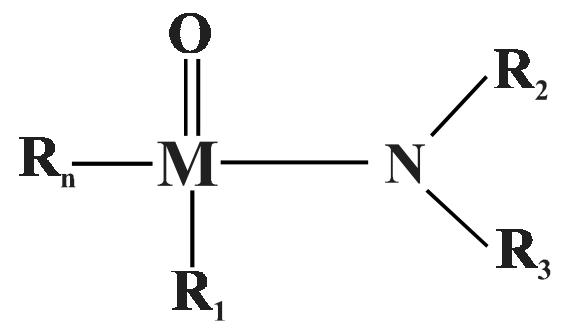

where:

$\mathrm{M}$ is a carbon, sulfur, or a phosphorous atom;

$\mathrm{R}_{2}$ and $\mathrm{R}_{3}$, are each a hydrogen atom or a lower alkyl group;

$R_{1}$ and $R_{n}$ are either each a lower alkyl group, another 

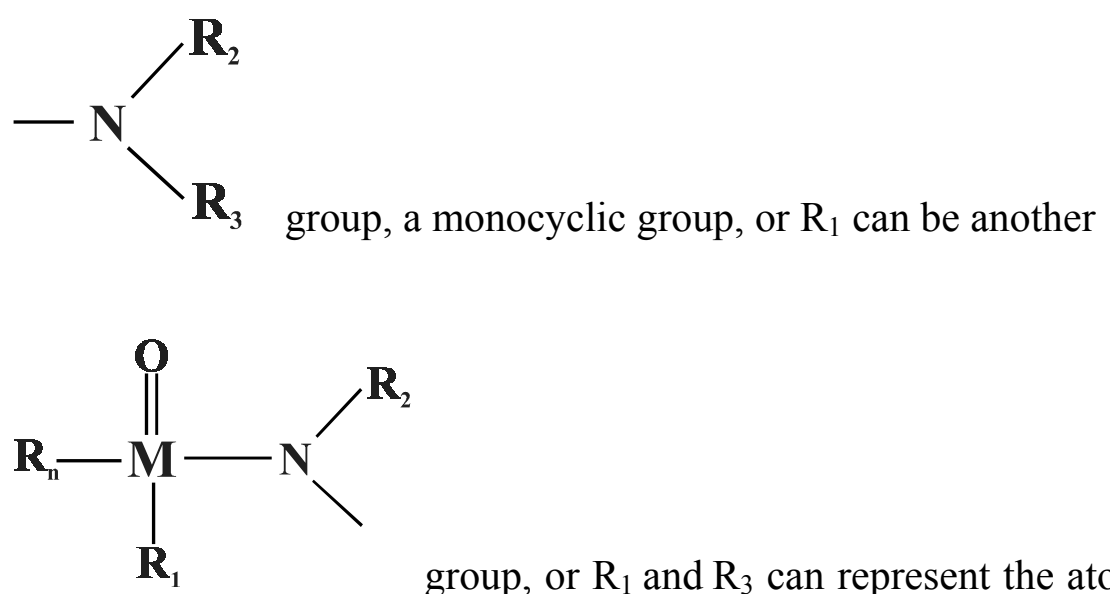

group, or $R_{1}$ and $R_{3}$ can represent the atoms necessary to close a heterocylic ring, and $n=1$ where $M=$ phosphorus and is otherwise 0 . Where $R_{n}$ and $R_{1}$ are either or both lower alkyl groups in this formula alkyl can apparently have a carbon content in the range of $\mathrm{C}_{1}-\mathrm{C}_{4}$ or possibly $\mathrm{C}_{5}$, of which $\mathrm{C}_{1}$ and $\mathrm{C}_{2}$ are considered preferable. Preferred substituents for $\mathrm{R}_{2}$ and $\mathrm{R}_{3}$ are methyl and ethyl groups, although it is presumed that homologs up to about $\mathrm{C}_{4}$ or possibly higher would produce more or less useful solvent compounds, and the replacement of such groups with one or more hydrogen atoms also appears to be an acceptable alternative. Monocyclic aromatic groups such as benzyl radical might also prove as a benzyl radical might also prove useful as the substituent $R_{n}$ and $R_{1}$, because the structure of this group is favorable to the resonance stabilizing function of the solvent. Either or both of $\mathrm{R}_{\mathrm{n}}$ and $\mathrm{R}_{1}$ can be another amino group" (Stiller et al., 1981).

Some examples of supersolvents are tetramethyl urea (TMU), dimethyl sulfoxide (DMSO), hexamethyl phsphoramide (HMPA), N-, N-dimethyl acetamide (DMAA), and N-methyl-2pyrrolidone (NMP) (Stiller et al., 1981).

\subsubsection{Studies of Supersolvents on Coal}

Studies of the effects of supersolvents on coal have shown that they are capable of extracting significant proportions of coal's organic matter at relatively mild extraction conditions.

Roy et al. (1978) studied the extraction of coal using DMSO as the solvent and found that its solvent capacity is comparable to that of any other good solvent such as pyridine or ethylenediamine.

Leong (1985) and Renganathan (1986) preformed one-hour extraction studies using TMU $\left(166^{\circ} \mathrm{C}\right)$ and NMP $\left(202^{\circ} \mathrm{C}\right)$ at a $10: 1$ solvent-to-coal ratio and obtained extraction yields as high as 50\% (MAF) for TMU and 70\% (MAF) for NMP. NMP has a higher boiling point $\left(202^{\circ} \mathrm{C}\right)$ than either TMU or DMSO. In addition NMP has been shown to swell coal much more than other solvents (Marzec et al. 1979). 
Renganathan (1986) investigated NMP's ability to reduce the ash and pyritic sulfur content of coal. Renganathan found that the ash content of various coals could be reduced to as low as $0.1 \%$ by simple extraction with NMP. Renganathan also observed that the pyritic sulfur content of the coal could be significantly reduced by extraction with NMP. The ability of NMP to remove both inorganic material and pyritic sulfur is due to its inability to dissolve appreciably these inorganic species under the mild extraction conditions utilized.

Phillips (1988) investigated the extraction yields of 10 different coals using NMP. Phillips concluded that the extraction is essentially complete after twenty minutes when operating above the temperature of $150^{\circ} \mathrm{C}$.

Gadam's (1990) preliminary experiments using NMP as a solvent proved that NMP is a superior solvent to DMSO. Gadam attributed the solvent power of NMP to the presence of the electron pairs on both the nitrogen and oxygen atoms.

\subsubsection{N-methyl-2-pyrrolidone (NMP), The Solvent of Choice.}

Stiller et al. (1985) and Renganathan (1988) used NMP as the solvent of choice for coal extraction experiments at West Virginia University. Through their experiments they concluded that NMP gives higher extraction yields than HMPA, TMU and DMAA. The research proposed here was based upon the results from previous research results. The advantages of using NMP as a solvent for coal extraction include: [Leong (1985), Stiller et al. (1985), Renganathan (1986), and Phillips (1988)]

1) High extraction yields $(65+\%)$ are possible at relatively mild conditions $\left(202^{\circ} \mathrm{C}\right.$ and atmospheric pressure) depending on the coal.

2) NMP extraction removes almost all the mineral matter and pyritic sulfur and part of the organic sulfur during the extraction.

3) Solvent and extract can be easily separated from the coal solution by precipitation with water or by solvent evaporation.

4) The solvent can be reused repeatedly.

5) NMP is a relatively safe solvent for use in coal extractions compared to the other classic coal solvents like: dimethyl sulfoxide (DMSO), hexamethyl phsphoramide (HMPA), N-, N-dimethyl acetamide (DMAA).

The important chemical and physical properties of NMP are shown in Table1.1 (GAF 1972). Material safety data sheet for NMP, are supplied by the manufacturer BASF.

Marzec et al. (1979) used the electron donor-acceptor theory to explain the effectiveness of NMP as a solvent for coal extraction. Marzec et al. modeled solvent extraction of coal as a series of replacement reactions of the coal-coal electron donor- acceptor bonds by the coalsolvent electron donor-acceptor bonds (Gadam 1990). This theory suggest that solvents with electron donor number $\left(\mathrm{D}_{\mathrm{N}}\right)$ greater than 19 and electron acceptor number $\left(\mathrm{A}_{\mathrm{N}}\right)$ less than 21 would be effective solvents for extraction. NMP has $\left(D_{N}\right)=27.3$ and $\left(A_{N}\right)=13.3$. This model can explain why NMP is an effective solvent. The strong solvating characteristic of NMP on various polar and nonpolar species is also believed to be due to the presence of lone 
electron pairs on the nitrogen and the oxygen atom (Gadam 1990). Furthermore, the NMP molecule is configured so that it changes its conformation depending upon the relative polarity of the surroundings. This change in configuration also changes the position of the lone pair electrons, which allows the solvent molecule to adapt its polarity to the polarity of its surroundings. This ability of the NMP molecule to adapt itself to its surroundings makes it an extremely powerful solvent for coal extraction where both polar and nonpolar species are encountered (Gadam 1990).

Table 1.1 Key Physical and Chemical Properties of N-Methyl-2-Pyrrolidone

\section{Structure}

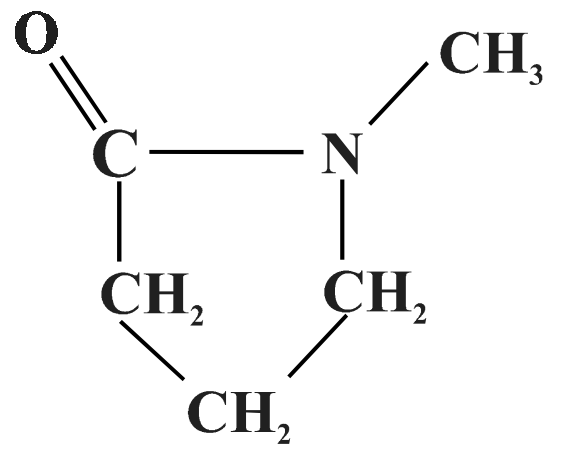

Empirical Formula:

Molecular Weight:

$\mathrm{C}_{5} \mathrm{H}_{9} \mathrm{NO}$

Physical form:

99.13

Color (APHA):

Liquid with mild amine-like odor.

Melting Point:

50

Boiling Point:

$-24^{\circ} \mathrm{C}\left(-11.9^{\circ} \mathrm{F}\right)$

$202^{\circ} \mathrm{C}\left(395^{\circ} \mathrm{F}\right) @ 760 \mathrm{mmHg}$

$150^{\circ} \mathrm{C}\left(302^{\circ} \mathrm{F}\right) @ 162 \mathrm{mmHg}$

$100^{\circ} \mathrm{C}\left(212^{\circ} \mathrm{F}\right) @ 24 \mathrm{mmHg}$

$\mathrm{T}^{\mathrm{sat}}=\left\{\ln \left(\mathrm{P}^{\mathrm{sat}}\right)+0.2349\right\} / 0.0156$

Viscosity $\left(25^{\circ} \mathrm{C}\right)$

$1.65 \mathrm{cp}$

$\left(\mathrm{T}^{\mathrm{sat}}\left[{ }^{\mathrm{o}} \mathrm{C}\right], \mathrm{P}^{\mathrm{sat}}[\mathrm{mmHg}]\right)$

Specific Gravity:

$1.027 @ 25^{\circ} \mathrm{C}$

$.987 @ 75^{\circ} \mathrm{C}$

$.969 @ 100^{\circ} \mathrm{C}$

Specific Heat (Cp):

$0.40 \mathrm{Kcal} / \mathrm{kg}$ at $20^{\circ} \mathrm{C}$

$\mathrm{Cp}_{\mathrm{NMP}}=8.04 * 10^{\wedge}-4 *(\mathrm{~T})+.38$

Thermal Conductivity (kNMP):

$\mathrm{kNMP}=-1 * 10^{\wedge}-4(\mathrm{~T})+0.1954$

(T $\left.\left[{ }^{\circ} \mathrm{C}\right], \mathrm{knmp}\left[\mathrm{W} / \mathrm{M} /{ }^{\circ} \mathrm{C}\right]\right)$

Heat of Vaporization

$127.3 \mathrm{~K} \mathrm{cal} / \mathrm{kg}$ at $20^{\circ} \mathrm{C}$

Interfacial Surface Tension $\left(25^{\circ} \mathrm{C}\right): 40.7$ dynes $/ \mathrm{cm}$

Flash Point (open cup):

$95^{\circ} \mathrm{C}\left(204^{\circ} \mathrm{F}\right)$ 
Dipole Moment

Dielectric Constant $\left(25^{\circ} \mathrm{C}\right)$ :

Solubility parameter ( ):

Miscibility with Other Solvents:
$4.09 \pm 0.04$ Debye

32.2

11.0

completely miscible with water and most organic solvents including alcohols, esters, ketones, aromatic and chlorinated hydrocarbons and vegetable oil.

\subsubsection{Parametric Study of Solvent Extraction}

The most important parameters that affect the amount of solubility of the coal are time and temperature of extraction. In addition to these two parameters, the particle size of the coal, the presence of moisture and oxygen in the coal, and pretreatment of the coal also affect the solvent extraction of coal to a certain extent. Oxygen degrades most solvents; consequently coal with high oxygen content is not commonly used. The most undesired component of coal is moisture (water). The presence of water in the solvent greatly reduces it solubility. Water can be a real problem, especially in a continuous process where the solvent is continuously recycled causing the water composition to rise decreasing the solubility of coal.

Some of the important aspects of the parametric study of solvent extraction are discussed below:

\subsubsection{Effect of Time and Temperature (Kinetics of Solvent Extraction)}

Several scientists have studied the kinetics of solvent extraction using several different solvents and many different coals. This research focuses on the extraction of the bituminous Upper Powleton coal (WVGS 13421) using N-methyl-2-pyrrolidone (NMP) as the solvent.

Phillips (1988) proposed first order irreversible kinetics for the extraction of Upper Eagle coal using NMP as the solvent and obtained a good fit of the initial rate data with this kinetic model. Philips also investigated NMP extraction of ten different coals and concluded that:

- NMP extraction yields for the ten coals range from 22.8\% (MAF) to $57.9 \%$ (MAF). Coals obtained from the Northern West Virginia coalfield tended to have a higher NMP extraction yields than those obtained from the southern West Virginia coalfield.

- The NMP extraction mechanism is believed to be limited by mass transfer and for temperatures less than $135^{\circ} \mathrm{C}$ due to decreased pore dimensions and availability of active sites for extraction. At temperatures greater than $135^{\circ} \mathrm{C}$ the extraction is limited by mass transfer effects only due to increased swelling of the coal in this temperature region. The increased swelling of the coal at these temperatures exposes more active extraction sites to the solvent resulting in increased extraction rates and yields. 
- NMP solvent extraction tends to decrease the BTU value, carbon, and hydrogen content of the residue while it increases the BTU value, carbon, and hydrogen content of the extract.

- NMP solvent extraction tends to increase the total sulfur and ash in the residue and decrease the total sulfur and ash of the extract.

- The activation energy obtained for the high-temperature extraction region (T > $135^{\circ} \mathrm{C}$ ) was $1.3 \mathrm{Kcal} / \mathrm{mol}$ and indicates that the extraction mechanism is controlled by diffusion alone in this temperature region. This value is in good agreement with predicted activation energies for diffusion in liquids.

- The initial rate of the NMP extraction and the ultimate extraction yield increases with increasing treatment temperature. At temperatures greater than $150{ }^{\circ} \mathrm{C}$ the extraction is essentially complete after twenty minutes.

Phillips observed that with increasing temperature the extraction yield leveled off at a higher value after a period of time. A similar observation was made by Renganathan (1986). Renganathan studied the NMP extraction of Bakerstown coal and found that after about 10 minutes of extraction time, the yield of extract leveled off. He observed a pseudo-first order kinetic behavior of Bakerstown coal and again fitted initial rate data. Gadam (1990) derived a $1^{\text {st }}$ order reversible kinetic rate model to explain the entire kinetics.

\subsubsection{Effect of Particle Size on Extraction Yield}

Researchers have noticed a significant increase in the extraction yield with the decrease in coal particle size to a certain extent. However, Kiebler considered that the rate of increase in yield with decrease in coal particle size is much too small to be accounted for on the assumption that a surface layer of constant thickness is completely extracted (Lowry, 1945). Renganathan (1986) studied the effect of coal particle size from 60 to 200-mesh on the extraction yield and found the results to be insignificant. This finding is very important from the process economics point of view, since with the conventional crushers coals can be crushed down to 60 mesh size and no grinding of coal (an expensive unit operation) would be required for this solvent extraction process (Gadam 1990).

\subsubsection{Effect of Oxygen and Moisture on Extraction}

The presence of oxygen during extraction also decreases the yield with most of the solvents (Dryden, 1950). The presence of moisture in coal has an unfavorable effect on extraction yield for solvents that are miscible with water. This is probably because water impedes the 
power of the solvent since water alone is not a good solvent for coal (Gadam 1990). Water greatly reduces coal's solubility in NMP. As little as 3-mole $\%(0.6 \mathrm{wt} \%)$ water will cut the extraction yield more than $50 \%$. This makes the issue of moisture content in coal extremely important, especially for a continuous process where the solvent would be recycled.

\subsubsection{Solvent Swelling of Coal}

Researchers have argued that the ability of a solvent to effectively take the organic matter from the coal into solution largely depends on the degree of penetration of the solvent into the organic matrix pore system. This basically states that a solvent's extraction ability is diffusion controlled. When contacted by a solvent, all but the most mature coals imbibe fluid, swell, and depending on the solvent and extraction conditions, will disperse and dissolve (Phillips 1988).

\subsubsection{What is the Product of Solvent Extraction of Coal?}

Solvent extraction of coal produces two materials - the extract and residue that can be generally characterized as:

$\begin{array}{llll}\text { Organic Material } & \text { Extract } & & \text { Residue } \\ \text { Mineral Sulfur } & \text { None } & & \text { Some } \\ \text { Inorganic Sulfur } & \text { None } & & \text { All pyrites \& sulfates } \\ \text { Organic Sulfur } & \text { Some } & & \text { Some }\end{array}$

Source: Zondlo 2000

The goal of this research task was to scale up the production of an ash-free coal-based carbon pitch (extract) from the laboratory scale to pilot scale using NMP as the extraction solvent. The product of this solvent extraction is a coal based carbon pitch referred to as "reconstituted coal" or extract. This coal-based product is essentially free of inorganic matter and pyritic sulfur.

The use of different types of solvents and coals produces a variety of coal based carbon pitches. However, supersolvents extraction on bituminous coals produce a relatively consistent coal extract. When the extraction process is complete, and the extracted coal materials are processed to dryness, a "solvent free" solid is produced. Several uses have been identified for this product. Some of the important ones are listed below (Gadam 1990):

1) As an environmentally "clean" fuel, since the ash and sulfur are removed.

2) As a feedstock for further hydrogenation and upgrading to higher quality fuels.

3) As a source of electrode carbon used in the aluminum industry.

4) As a base for varnish manufacture.

5) As a filler in rubber compounding.

6) Addition of coal extracts in noncaking coals to induce caking.

7) Briquette binding.

8) Production of pitch containing little "free carbon". 
9) Production of colloidal fuel.

10) Production of low ash carbon.

11) Production of special grades of jet fuels for supersonic aircrafts (Wise,1971).

12) Precursor for the production of Carbon Foam.

13) Ingredient to make graphite, coke, binder pitch, and impregnation pitch.

Zondlo (2000) presented a few uses for the extract and its related values at a presentation to Dupont and New Carbon at Morgantown, West Virginia. These uses and values can be seen in Table 1.2.

Table 1.2 Potential Uses of Coal Extracts

\begin{tabular}{|c|c|c|}
\hline Products & Customers & $\begin{array}{c}\text { Current Market Price } \\
\text { (approx.) }\end{array}$ \\
\hline \multicolumn{3}{|l|}{ Fuel } \\
\hline Low-ash, Low-sulfur & Power Generation & $\$_{3-6 / 10^{6} \mathrm{BTU}}$ \\
\hline \multirow{2}{*}{ Solid Fuels } & Transportation & \\
\hline & \& Domestic Heating & \\
\hline \multicolumn{3}{|c|}{ High-grade Coke } \\
\hline Electrodes & Steel Industry & $\$_{1 / 1 b}$ \\
\hline Anodes & Aluminum Industry & ${ }^{\$} 0.18 / \mathrm{lb}$ \\
\hline Graphite & Nuclear Reactors & $\$ 10 / \mathrm{lb}$ \\
\hline \multicolumn{3}{|l|}{ Pitch } \\
\hline Binder & Aluminum \& Steel Industry & ${ }^{s_{0}} .15 / \mathrm{lb}$ \\
\hline Impregnation & Graphite Industry & ${ }^{{ }_{0}} 0.15 / \mathrm{lb}$ \\
\hline \multirow[t]{2}{*}{ Mesophase (fiber) } & Nuclear Reactor & $\$_{1-5 / l b}$ \\
\hline & Military \& Aerospace & \\
\hline
\end{tabular}

\subsection{Experimental}

\subsubsection{Comparison of Laboratory Procedure and Pilot Scale Operation}

As described earlier, the major effort in this research was the design and construction of a larger pitch production facility to manufacture significant amounts of reconstituted coal. The change in dimension can only be appreciated when consideration is given to the previous laboratory procedure. The following section details the previous (laboratory scale) production method, and provides an overview of the scaled-up process (CEPU) with its major steps and equipment. Also the design and construction of the pilot unit is laid out, and the startup is discussed.

\subsubsection{Laboratory Scale Pitch Production}

In the laboratory procedure (shown as a Block Flow Diagram in Figure 1.1), samples of coal are ground to $\leq 60$ mesh $(0.25 \mathrm{~mm})$. After grinding, the coal is dried under a 


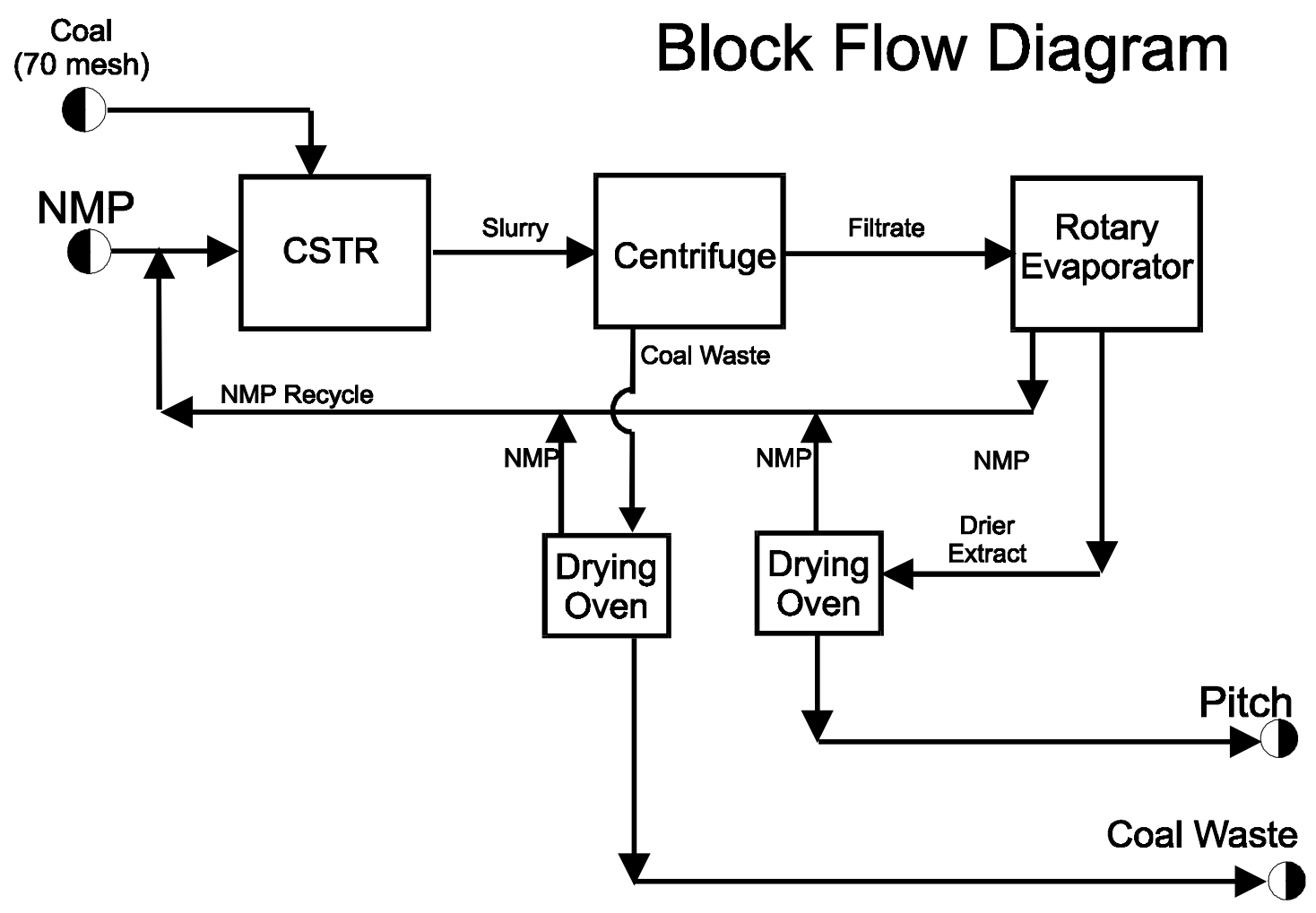

Figure 1.1 Block Flow Diagram for Laboratory Production of Coal-Based Carbon Pitch

Nitrogen atmosphere (drying in the presence of air oxidizes the coal and decreases its solubility). The coal is dried because water diminishes the ability of NMP to dissolve coal. The coal is then mixed with the aprotic dipolar solvent, N-Methyl-2-pyrrolidone (NMP), at a ratio of 1 part coal to 10 parts NMP by weight. The coal/NMP slurry is then heated to $202^{\circ} \mathrm{C}$, the boiling point of the solvent. The mixture is kept at this temperature for 1 hour. Prior experimentation has shown that in this time, the maximum portion of the coal is dissolved in the solvent. In a laboratory setting, the dissolution step is done in a 5 gallon stirred tank reactor (CSTR) under a nitrogen atmosphere to prevent any degradation of the solvent. The slurry is then permitted to cool to room temperature. The slurry is then centrifuged to separate the dissolved coal/NMP liquor from the undissolved solid coal/ash. In the laboratory, this centrifugation is done in four symmetrically arranged $750 \mathrm{ml}$ centrifuge containers. It requires 25 minutes at $2000 \mathrm{~g}$ to separate all the ash. After centrifugation, the supernatant containing all the soluble coal material and the NMP is decanted off. The residue that contains all the ash and insoluble coal is removed from the centrifuge flasks and the process is repeated until the 5 gallons of slurry are centrifuged.

The supernatant is then transferred to a rotary evaporator (Rotovap) flask. The rotary evaporator is essentially a batch thin film evaporation unit. While the capacity of the flask 
is 20 liters, only 8 -liter batches of solution can be run at a time. The pressure in the Rotovap is reduced to less than $1-\mathrm{mmHg}$ using a vacuum pump. The flask is rotated in a hot $\left(110^{\circ} \mathrm{C}\right)$ oil bath to heat the mixture. The vapor pressure of the solvent is high enough at this temperature to evaporate off the NMP, which is then condensed using a water-cooled heat exchanger. The condensed NMP is collected for recycle. The evaporation procedure is done until no more condensate (NMP) is given off for $1 / 2$ hour. The required time for evaporation depends upon the dissolved coal-to-solvent ratio. As the solvent is removed, the dissolved coal coats the inside surface of the rotating flask creating a thick skin. This skin reduces the heat transfer rate, thus lengthening the batch time.

After the procedure is completed, the NMP is recovered and recycled back to the reactor for another run. The flask containing the dried coal extract is removed from the Rotovap, filled with a nitrogen atmosphere, and sealed. The sealed flask is put in a freezer. At low temperatures the solid coal extract becomes friable and can be "chipped" from the internal surface of the flask. However, at this condition the coal extract is still not completely free of NMP and an additional drying step is needed.

The additional drying step is required to lower the solvent concentration in the coal extract below the level of detection. Once removed, the chipped coal extract is crushed to $\leq 40$ mesh $(0.425 \mathrm{~mm})$ and placed in trays in a vacuum drying oven. The drying oven temperature is set at $205^{\circ} \mathrm{C}$ and the system is evacuated to about $50 \mathrm{mmHg}$. In addition, a low flow rate of preheated nitrogen is permitted to flow over the drying chipped coal extract. The nitrogen carries the NMP out of the oven and into a cold trap installed just before the vacuum pump. This drying process takes over 18 hours. The NMP is again recovered for recycle. The product of this step is the dry coal extract, or pitch, that will be used for subsequent experimentation.

Since NMP is a very expensive solvent $(2.12 \$ / 1 b)$, it is imperative that as much as possible be recovered and recycled. The residue (insoluble coal and ash) from the centrifuge is rich in NMP - nominally it contains about 55\% by weight NMP! NMP is recovered by vacuum drying the residue in the same oven as the extract (described above). This operation also requires about 18 hours. The products of this separation are a high-surface-area coal and NMP. The NMP is again recycled for another run.

Limited by the size and multi-step sequential batch nature of the laboratory preparation process, only a limited supply of coal extract could be prepared. It would take one lab technician 40 hours to make 600 grams of pitch. Therefore, the time required to make 10 $\mathrm{kg}$ of pitch would be greater than 6 months - providing that no equipment failed! The most fragile piece of equipment is the Rotovap. The Rotovap is mostly glass. It is also the most fragile and expensive piece of equipment to fix. Because it is manufactured in Sweden, replacement parts require about 6 to 8 weeks to arrive.

With equipment and personnel limitations, it would be impracticable to proceed with the manufacturing of large quantities of coal extract using the laboratory procedure. A larger experimental operation needed to be designed and constructed to increase efficiency. This facility could also be used to evaluate the economics of the processing steps required for 
mass production of the coal-based carbon pitch. 


\subsubsection{Pitch Production in the Coal Extraction Pilot Unit (CEPU)}

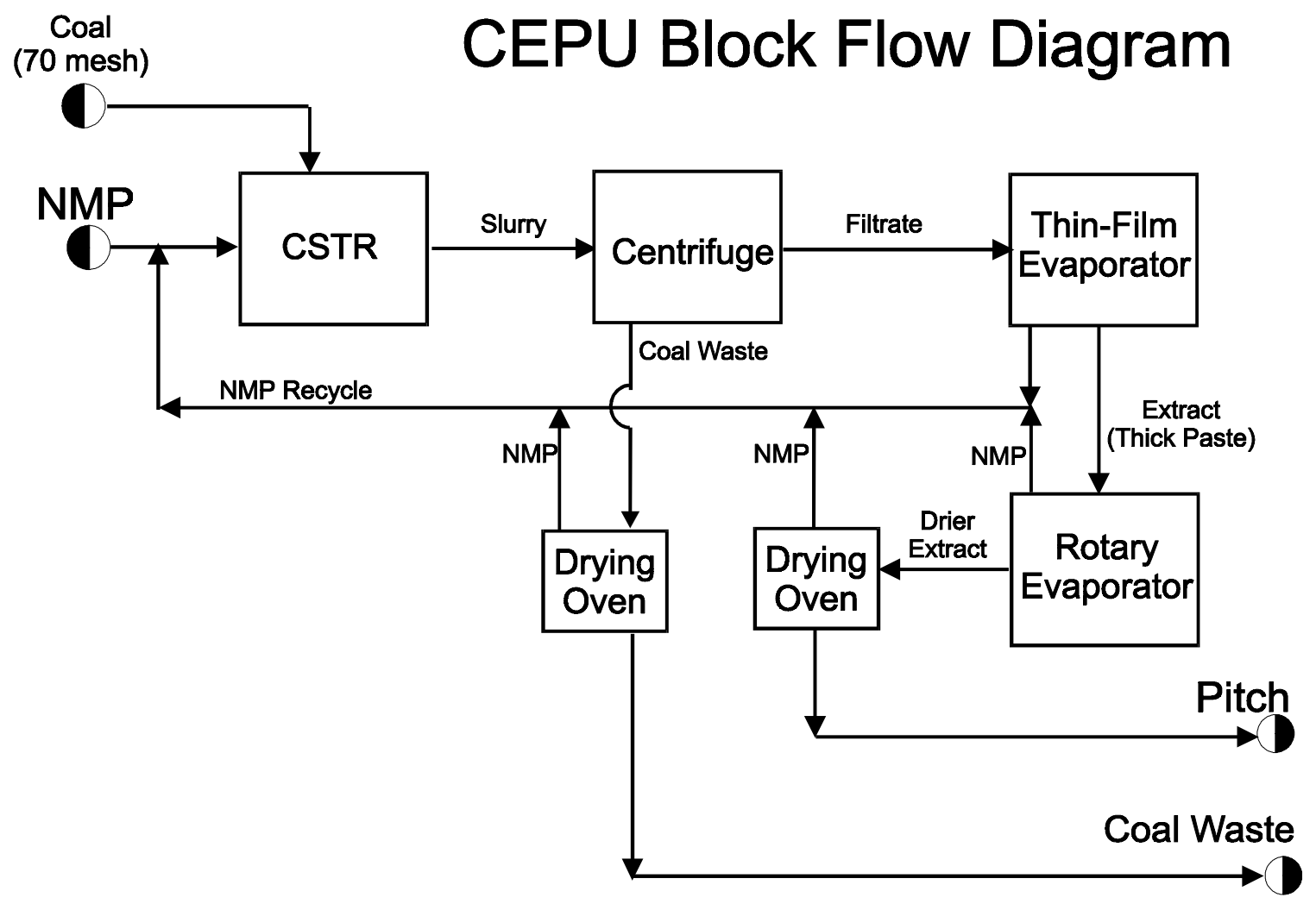

Figure 1.2 Block Flow Diagram for Pilot Plant Production of Coal-Based Carbon Pitch

Just prior to the beginning of research on this project, the feasibility of constructing a pilot plant was discussed. The size of the pilot plant equipment would be significantly larger than that used in the laboratory operation. Therefore, more substantial commercial type equipment was to be incorporated into the pilot plant design. A Block Flow Diagram of the pilot plant is shown in Figure 1.2. Instead of a 5-gallon CSTR, a 25-gallon CSTR was to be the extraction unit. Instead of a laboratory centrifuge that had a maximum capacity of four $750 \mathrm{ml}$ flasks and could only reach $2000 \mathrm{~g}$, a small commercial continuous centrifuge that had the capacity of 5-gallon per minute at $2170 \mathrm{~g}$ was utilized.

In the pilot unit the supernatant, which previously went to the Rotovap for solvent removal, is instead sent to a wiped thin film evaporator (TFE). The TFE removes about $70 \%$ of the NMP at a feed rate of about 0.5 -liter/minute. With a $70 \%$ reduction in solvent, the product leaves the TFE as a thick paste. The majority of the remaining NMP is removed from the thickened extract in a modified rotary evaporation unit. The Rotovap takes the remaining $30 \%$ of initial NMP and reduces it down to $5 \%$. The product leaving the Rotovap contains $\leq 5 \%$ by weight NMP, and is semi-solid. The Rotovap drying process is followed by a vacuum drying step (as described previously) to remove the remainder of the NMP. In each step, NMP is collected and recycled back to the reactor for another run.

This process is "essentially" the same as the one previously performed in the lab. The 
primary difference is that $70 \%$ of the solvent is now driven off before the Rotovap by the TFE. A few modifications were made. (1) The Rotovap was improved to increase its performance. In the pilot unit the flask is an old converted 20-liter low-pressure steel Freon tank. With a little machining the tank was made to fit the Rotovap. The larger metal flask facilitates better heat transfer and will permit the drying of larger volumes (14 liters) of thickened sample than the glass vessel. The metal tank also allows more "physical" means of removing the semi-dried extract than the glass. With these modifications, the time required per weight of product is significantly decreased. (2) The larger drying ovens used in the pilot unit have capacities 30 times greater than the laboratory models.

The pilot plant can produce up to $25 \mathrm{~kg}$ of pitch in a week under the supervision of two technicians. This is compared to the $0.6 \mathrm{~kg}$ pitch a week in the lab by one technician. This facility was also used to evaluate the economics of the processing steps required for mass production of the coal-based carbon pitch.

\subsubsection{Construction of the Coal Extraction Pilot Unit}

The construction of a pilot unit was not a simple operation. First, a suitable floor space was required. The mezzanine floor in the high-bay area in the National Research Center for Coal and Energy (NRCCE) was vacant and suitable. After the water, electrical, and safety components were in place, the pilot plant construction was started.

\subsubsection{Pilot Plant Construction Timeline}

Every construction operation needs a timeline so parallel steps can be planned and completed in a timely manner. The construction of the pilot plant was a major operation. Therefore, a timeline was made up to keep everything on schedule.

This project was divided into five main parts. The first part of construction was to design and manufacture the individual components of the CEPU. The second part of the project was to create a detailed Chemical Hygiene Plan to help design the CEPU into an inherently safe working system. The third part was to prepare the laboratory area for the CEPU, and assemble the components. The fourth part of the research project was to assemble, operate, and debug the system so as to permit convenient production of significant quantities of coalbased carbon pitch. The fifth part of the research project to generate data on mass and energy balances. Inherent in this part are the evaluation with respect to time constraints on the basic unit operations involved in pilot plant operations. These five main parts, listed below, are further broken down into tasks. The timeline for each task is shown in the Time Task Deliverable Chart in Figure 1.3.

1) CEPU Equipment Design

2) Safety Concerns and Chemical Hygiene Plan

3) Mezzanine Preparation and Component Manufacturing \&Assembly

4) CEPU Startup

5) Collect data necessary for Material and Energy Balance

6) Evaluate efficiencies of each Process Step and recommend changes
Task 1

Task 2

Task $3,4,5,6$

Task 7,8 ,

Task 9,10

Task 11, 
12

7) Implement Changes and evaluate Effectiveness

Task 13,14

8) Make Large Quantities of Extract

Task 15

Task 1: Design

Dec 1999 - June 2000

Task 2: Chemical Hygiene Plan

Feb 2000 - June 2000

Task 3: Electrical System Design \& Construction

Mar 2000 - July 2000

Task 4: Floor Drain and Containment System Construction Mar 2000 - July 2000

Task 5: Equipment Manufacturing

April 2000 - Aug 2000

Task 6: Equipment Assembly

June 2000 - Oct 2000

Task 7: $\quad$ NMP Run $1^{\text {st }}$

Aug 2000 - Oct 2000

Task 8: $\quad$ NMP Coal Run $1^{\text {st }}$

Aug 2000 - Oct 2000

Task 9: $\quad$ Mass Balance

Nov 2000 - Jan 2001

Task 10: Energy Balance

Nov 2000 - Jan 2001

Task 11: Evaluate Efficiencies

Feb 2001 - April 2001

Task 12: Recommend Changes

April 2001 - May 2001

Task 13: Implement Changes

May 2001 - July 2001

Task 14: Evaluate Effectiveness

July 2001 - Sep 2001

Task 15: Manufacture Materials

Aug 2001 - Nov 2001

Figure 1.3 CEPU Construction Time Task Deliverable Chart 


\subsubsection{CEPU Equipment Design}

The CEPU equipment can be divided into five main components listed below, as seen on the Block Flow diagram in Figure 1.2. The following five main units and their supporting components were scaled, designed and constructed to handle the $100 \mathrm{~kg}$ of NMP with $10 \mathrm{~kg}$ of coal per run.

1) Continuous Stirred Tank Reactor (CSTR)

$(\mathrm{R}-101)$

2) Centrifuge

(Ct-101)

3) Wiped Thin Film Evaporator (TFE)

(TFE-101)

4) Rotary Evaporator (Rotovap)

5) Drying Oven

(DO-101-3)

These main components and their supporting equipment are described in detail in the next section. The components are labeled with equipment numbers that relate to their position on the Process Flow Diagram (PFD). The PFD for this process can be seen in Figures 1.4 $\&$ 1.5. A stream table follows in Table 1.3, and an equipment list in Table 1.4. A Block Flow Diagram (BFD) with stream compositions is also shown in Figure 1.14.

\subsubsection{Safety Concerns and Chemical Hygiene Plan}

The designated location for the construction and operation of the CEPU is the mezzanine floor in the high bay of the National Research Center for Coal and Energy (NRCCE) building. In order to locate the CEPU at this site, a chemical hygiene plan (CHP) had to be prepared, reviewed by the safety management staff of the NRCCE and the West Virginia University Environmental Health and Safety office prior to any equipment being moved into the building. Only after an approved CHP was received did the construction of the CEPU begin. The CHP was written and approved before this work began.

\subsubsection{Mezzanine Preparations and Component Manufacturing Assembly}

The CEPU was constructed on the mezzanine in the following steps:

1. Designs of the water and electrical systems were drawn up. The specific electrical requirements for each of the components (pumps, heaters, motors, ...etc.) were needed, along with a detailed layout of the placement of the equipment. Contracts were prepared, sent out on bid, and awarded. WVU employees plumbed the water system. Tri County Electric was hired as the certified electrician to install the electrical wiring to meet all electrical codes.

2. The mezzanine was fitted for safety by installing a water supply system, eyewash, fire extinguishers, drainage system, and contained ventilation system.

3. The spill safety consisted of secondary and tertiary containment structures. 
The containment systems and additional components were designed, purchased, and installed.

4. Each piece of equipment had its own support structure(s) designed and manufactured. Detailed drawings were drafted and delivered to a qualified local machine shop to perform the work. The structures were designed to safely support each unit. The structures are easily disassembled and mobile.

5. Once the site preparation was complete, each unit was moved to the mezzanine by the overhead crane.

6. Finally, the wiring and plumbing for each piece was installed connecting the individual units into a safe operating system. This system was named the Coal Extraction Pilot Unit (CEPU). A complete Process Flow Diagram can be seen in Figures 1.4 and 1.5.

Most of the up grading of the mezzanine was done just prior to the start up of this work.

\subsection{CEPU Startup}

After the site construction was complete, and the equipment assembled, the CEPU was ready for startup. A large part of the time spent on this research was to operate and debug the system to allow convenient production of significant quantities $(3.5 \mathrm{~kg})$ of coal-based carbon pitch from the CEPU. For this step, each unit of the system was first batch tested using NMP alone. It was then tested using coal and NMP.

\subsubsection{First and Second Run With NMP}

The first two runs were done with just NMP to test for leaks, clean the units, develop pump curves, and rate test flow meters. This trial run helped to yield partial operating parameters for each component of the system up to the TFE. Since no coal was use, no separation in the TFE was needed. Experimentation with the TFE followed these tests when coal was used. The NMP from the initial trial runs was then sent to a Rotary Evaporator where the NMP was cleaned up by distillation to be reused.

\subsubsection{First Run With NMP and Coal}

After the NMP from the first two runs was cleaned, and all improvements and corrections were made to the system, a third run of coal and NMP (10kg coal $/ 100 \mathrm{~kg}$ NMP) was loaded into the CSTR. This run helped to develop more accurate operating procedures and parameters. Once the first coal/NMP run was completed, the system performance was evaluated.

\subsubsection{TFE Startup}


A Wiping Thin Film Evaporator (TFE) is used as the third stage of the CEPU process for manufacturing reconstituted coal. The solvent (NMP) in the CSTR (R-101) dissolves the coal. The dissolved portion of the coal is separated from the un-dissolved portion in the Centrifuge (Ct-101). The TFE is then used to evaporate the majority (72 $\pm 2 \%)$ of the solvent from the dissolved portion of the coal. Finally a Rotovap is used to drive off the most of the remaining solvent from the product before it is placed in a vacuum drying oven to complete the process.

A Pfaudler Wiping Thin Film Evaporator was purchased for this research project. Communication with the manufacture assured the group that the evaporator would be well suited to the extraction process. Experimental results showed that 10 gallon/hour could be processed through the unit. However, the unit was designed for liquid/liquid separation (separating a volatile liquid from a non or less volatile one). The TFE was not designed, and had not been used to separate a liquid from a solid. Therefore, the unit was designed and set up to only drive off a percentage of the solvent so that the product would still be a fluid and flow like a "thick liquid".

The TFE evaporator system came in two separate units: the first unit is the TFE itself (TFE101); and the second unit is a heater (HE-102), which provides the necessary heat to the TFE for the solvent evaporation. The $20-\mathrm{kW}$ heater unit came assembled and only needed to be fitted to the evaporation unit. The TFE evaporation system is also assisted by a vacuum pump (VP-101) to reduce the temperature at which the NMP is evaporated.

Again, the TFE evaporation system thickens the filtrate (solvent and dissolved coal) by separating a majority of the solvent through an evaporation process from the filtrate.

\subsubsection{Drying Ovens and Rotovap First Runs}

The CEPU has been described as being too big to be a laboratory-scale unit but too small to be a production plant. Certain pieces of equipment were not available in the size needed for the pilot plant. Therefore, the Rotovap and drying oven from the lab had to be used. Due to the large increase in material processed by each unit, some modifications had to be made.

The Rotovap was not modified significantly. The only major modification was the addition of a steel vessel instead of the glass one as explained in section 1.4.8. The drying ovens had never been used to dry any significant amount of material. Three vacuum drying ovens were utilized by the CEPU. As a result of the large amount of material being processed, a special condensing system (HE-105) was installed for the ovens as described in section 1.4.9. 


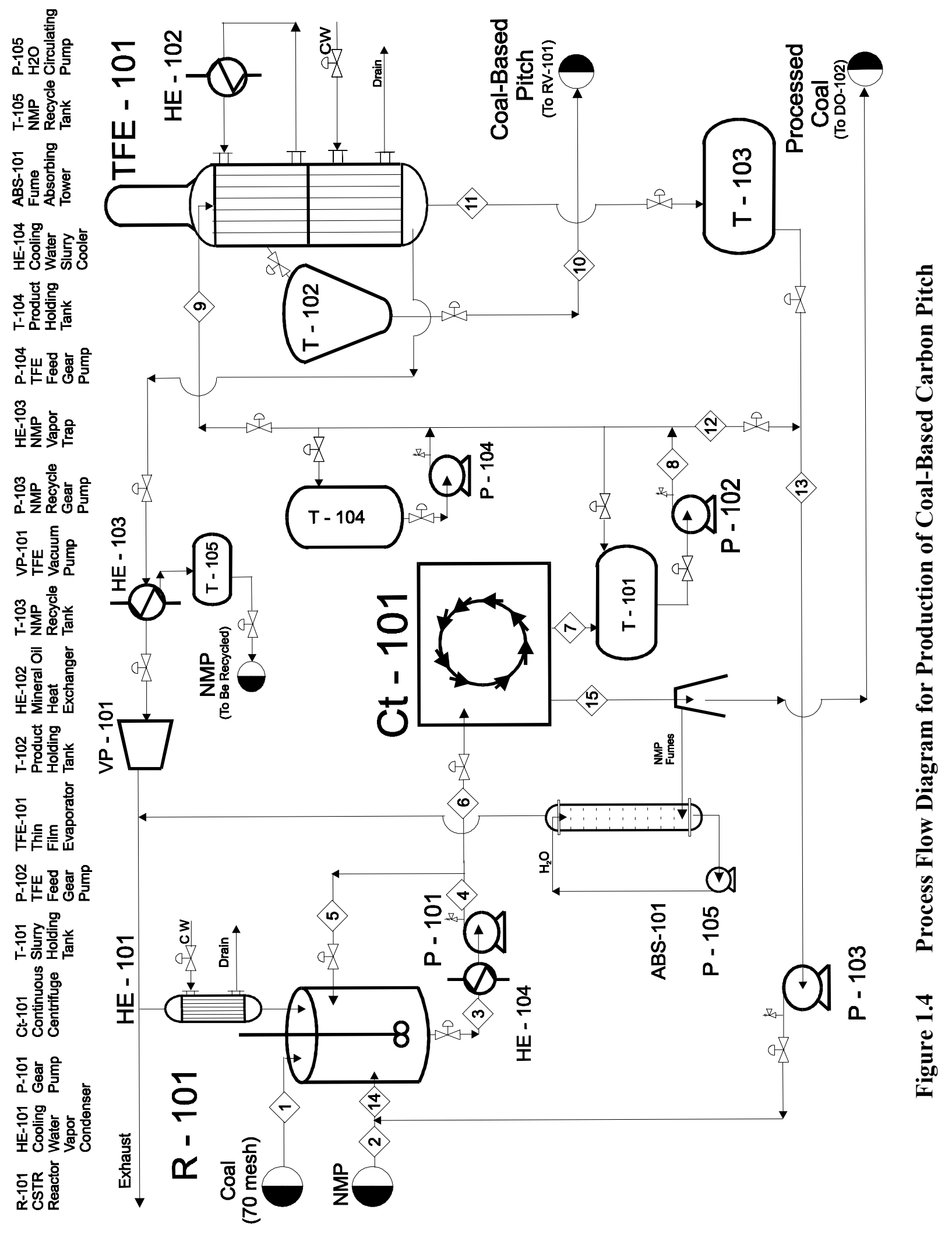




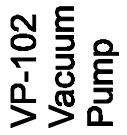

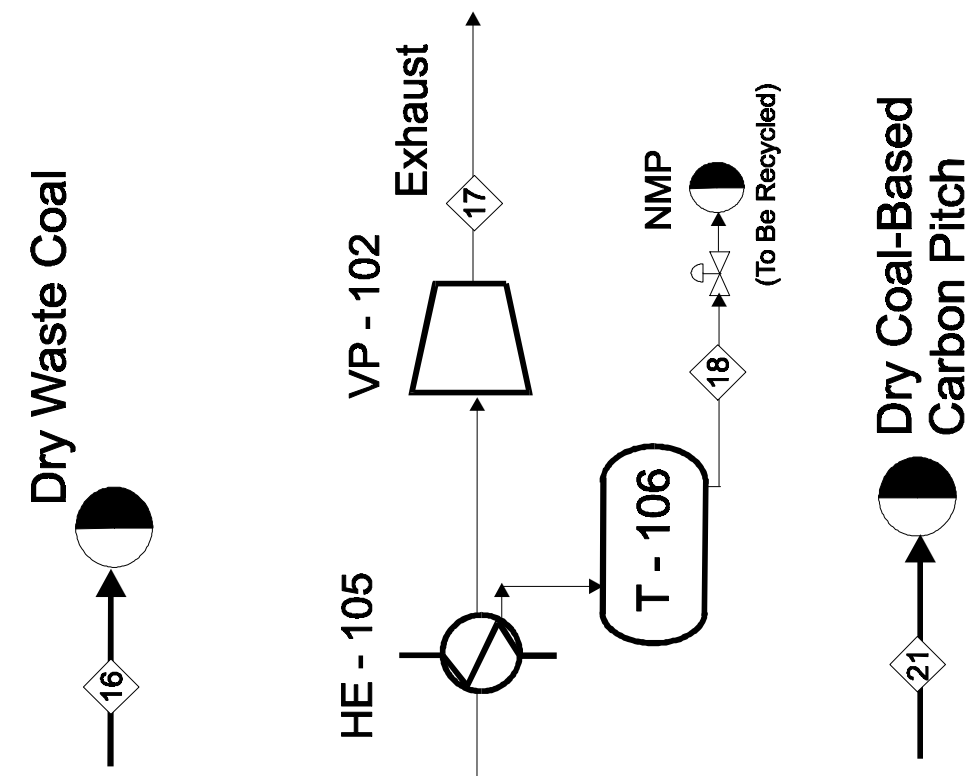

ํㅣㄴ

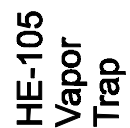

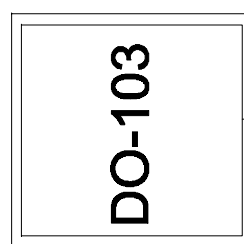

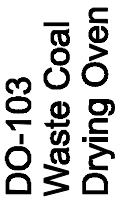

ㅇํㅇ

원

ํำ

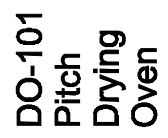

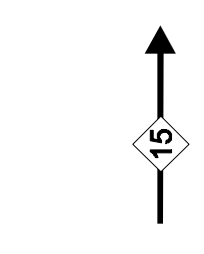

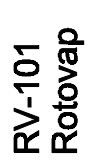
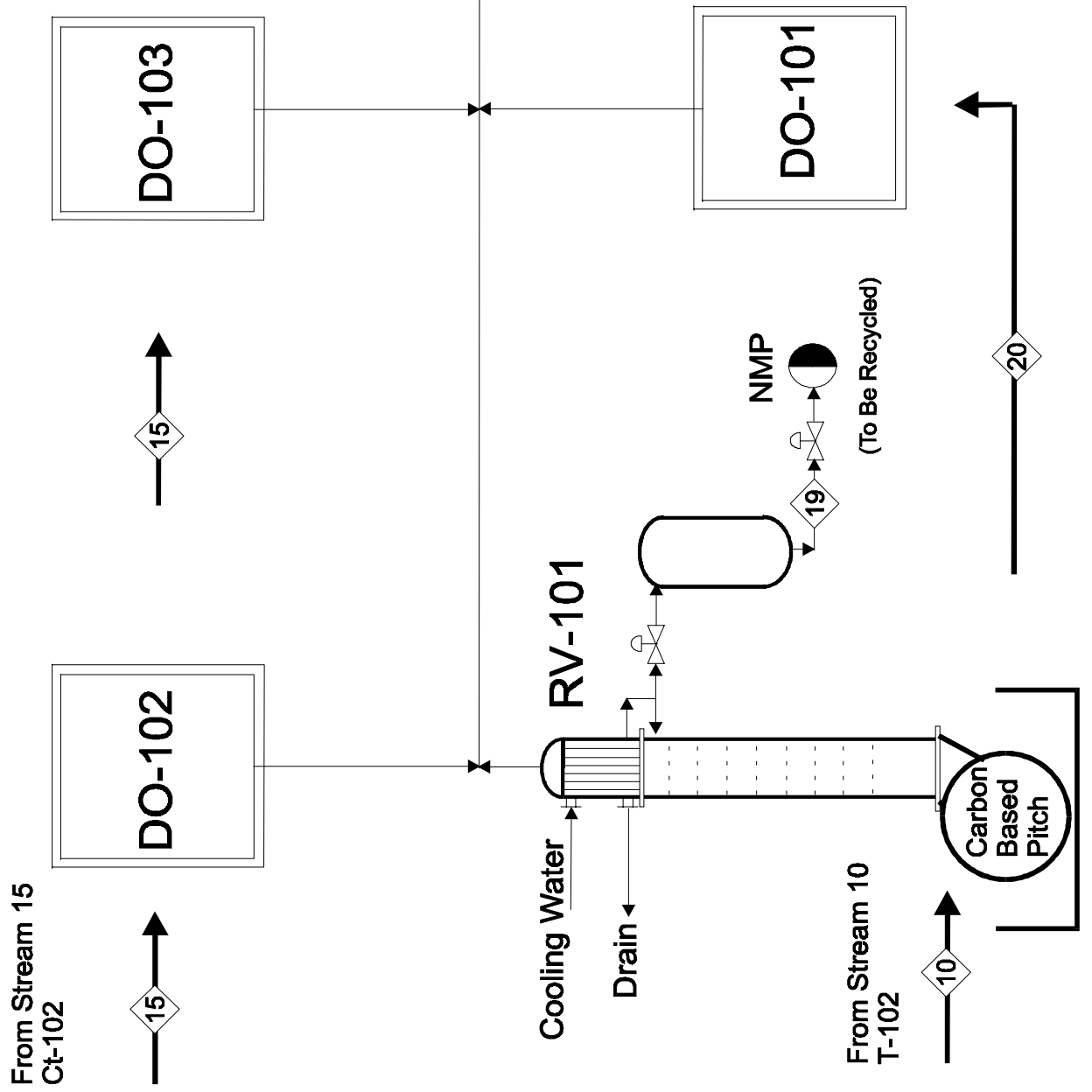

:

no 
Table 1.3 Coal Extraction Pilot Unit Stream Table

\begin{tabular}{|c|c|c|c|c|c|c|}
\hline \multirow[b]{3}{*}{ Stream } & \multicolumn{3}{|c|}{ Operating } & \multicolumn{3}{|c|}{ Stream Composition } \\
\hline & \multicolumn{2}{|c|}{ Temp } & \multirow{2}{*}{$\begin{array}{l}\text { Press } \\
\text { (psig) }\end{array}$} & \multirow{2}{*}{$\frac{\text { NMP }}{(w t \%)}$} & \multicolumn{2}{|c|}{$\underline{\text { Extract }} \underline{\text { Coal Waste }}$} \\
\hline & $\left({ }^{\circ} \mathbf{F}\right)$ & $\left({ }^{\circ} \mathrm{C}\right)$ & & & $(w t \%)$ & $(w t \%)$ \\
\hline 1 & 80 & 25 & & & Fresh & Coal ground to $\geq 60$ mesh \\
\hline $2 *$ & 80 & 25 & & & & \\
\hline 3 & 396 & 202 & & 90 & 3.5 & 6.5 \\
\hline 4 & 396 & 202 & $<60$ & 90 & 3.5 & 6.5 \\
\hline 5 & 396 & 202 & $<60$ & 90 & 3.5 & 6.5 \\
\hline 6 & 396 & 202 & $<60$ & 90 & 3.5 & 6.5 \\
\hline 7 & 396 & 202 & $<60$ & 96.3 & 3.7 & \\
\hline 8 & 396 & 202 & $<60$ & 96.3 & 3.7 & \\
\hline 9 & 396 & 202 & vac & 96.3 & 3.7 & \\
\hline $10 * *$ & 100 & 202 & vac & 88 & 12 & \\
\hline 11 & 70 & 25 & vac & 100 & & Recycle Stream \\
\hline 12 & & & $<60$ & 100 & & Recycle Stream \\
\hline 13 & 396 & 202 & & 100 & & Recycle Stream \\
\hline 14 & 396 & 202 & 20 & 100 & & Recycle Stream \\
\hline $15 * * *$ & 396 & 202 & & 55 & & 45 \\
\hline 16 & Waste & Coal & & $<0.1$ & & $>99.9$ \\
\hline 17 & Exhau & & & & & \\
\hline 18 & & & & 100 & & \\
\hline 19 & & & & 100 & & \\
\hline 20 & & & & 50 & 50 & \\
\hline 21 & Pitch & & & $<0.1$ & $>99.9$ & \\
\hline
\end{tabular}

* This stream table allows for a $0.1 \%$ loss of solvent (NMP) per pass.

** Stream 10 will be transferred to the Rotovap (RV-101) where the NMP will be extracted down to a concentration of less than $50 \mathrm{wt} \%$. Drying oven (DO-101) will bake off all of the remaining NMP. The black, powdery pitch will then be stored for future use, and the NMP recycled.

*** Stream 15 will be transferred to drying ovens (DO-102 \& 103) where the remaining NMP will be extracted down to a concentration of less than $1 \mathrm{ppm}$ NMP. The processed coal will then be in powdered form again. The only difference is the used coal will have a higher ash (mineral) content.

No clean up process shown on PFD for the $\mathrm{H}_{2} \mathrm{O}$ /NMP condensed by the reactor condenser (HE-101). 
Table 1,4 Equipment List for Coal Extraction Pilot Unit

\begin{tabular}{|c|c|c|c|c|c|c|c|c|c|}
\hline \multirow[b]{2}{*}{$\begin{array}{c}\text { Vol } \\
\text { e) (gal) } \\
\end{array}$} & \multicolumn{2}{|c|}{ SufaceArea } & \multicolumn{2}{|c|}{ Oper_Press } & \multicolumn{2}{|c|}{ Oper.Temp } & \multicolumn{3}{|c|}{ HowRate } \\
\hline & $\begin{array}{c}\text { Inside } \\
\text { (ft) }^{2}\end{array}$ & $\begin{array}{c}\text { Outside } \\
\left(\mathrm{ft}^{2}\right)\end{array}$ & $\begin{array}{c}\text { Mhx } \\
\text { (psia) }\end{array}$ & $\begin{array}{l}\text { Norm } \\
\text { (psia) }\end{array}$ & $\begin{array}{c}\text { Mhx } \\
\text { ('F) }\end{array}$ & $\begin{array}{l}\text { Norm } \\
\text { ('F) }\end{array}$ & $\begin{array}{c}\text { Mn } \\
\text { (gal } / \mathrm{min})\end{array}$ & $\begin{array}{l}\text { Max } \\
\text { (gal } / \text { min }\end{array}$ & $\begin{array}{l}\text { Norm } \\
\text { (gal } / / \mathrm{min})\end{array}$ \\
\hline 40 & & & 19.6 & 14.6 & 900 & 396 & & & \\
\hline & & & 14.6 & 14.6 & 210 & 200 & 0 & 5 & 1 \\
\hline & 1.2 & 1.3 & 295 & $<0.2$ & 650 & 408 & 0.2 & 2 & 0.5 \\
\hline 3 & & 6.5 & 14.6 & $<0.2$ & 356 & 230 & & & \\
\hline & & & 14.6 & $<0.2$ & 500 & 410 & & & \\
\hline & & & 14.6 & $<0.2$ & 500 & 410 & & & \\
\hline & & & 14.6 & $<0.2$ & 500 & 410 & & & \\
\hline & & & 14.6 & 14.6 & & 80 & 5 & 36 & 15 \\
\hline & 11.5 & 16.2 & 150 & 14.6 & 500 & 396 & & & \\
\hline & & & 50 & 35 & 600 & 408 & & & \\
\hline 5 & 24 & & 150 & $<0.2$ & 500 & -15 & & & \\
\hline & 7.3 & 7.85 & 60 & 20 & 600 & 396 & 0.38 & 4.9 & 3 \\
\hline & 15.5 & 19.6 & 75 & $<0.2$ & 400 & -15 & & 17 & 3 \\
\hline & & & 200 & 20 & 525 & 396 & 0.38 & 4.9 & 3 \\
\hline & & & 200 & 20 & 525 & 200 & 2.5 & 19.5 & 4 \\
\hline & & & 25 & 5 & & & & 17 & 10 \\
\hline & & & 200 & 20 & 525 & 200 & 2.5 & 19.5 & 4 \\
\hline & & & 40 & 30 & & 80 & & 20 & 8 \\
\hline & & & 14.6 & $<0.2$ & & & & & \\
\hline & & & 14.6 & $<0.2$ & & & & & \\
\hline 60 & & & 120 & 14.6 & & & & & \\
\hline 30 & & & 120 & $<0.2$ & & & & & \\
\hline 60 & & & 120 & $<0.2$ & & & & & \\
\hline 60 & & & 120 & 14.6 & & & & & \\
\hline 5 & & & 120 & $<0.2$ & & & & & \\
\hline 20 & & & 120 & $<0.2$ & & & & & \\
\hline
\end{tabular}




\subsubsection{Continuously Stirred Tank Reactor (R-101)}

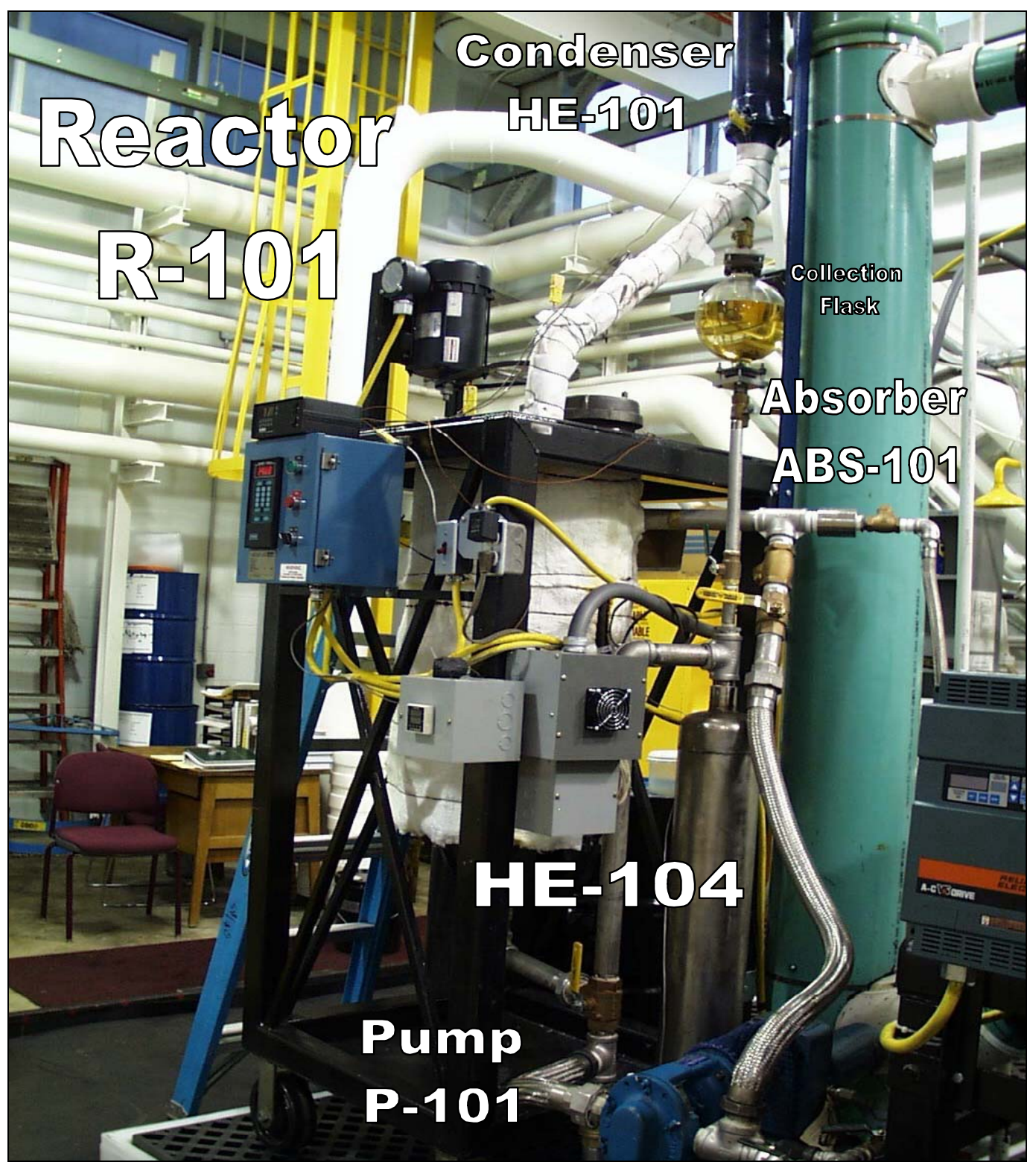

Figure 1.6 Continuous Stirred Tank Reactor with Heat Exchanger, Pump, Condenser, and Absorber

A suitable batch size for our unit is 100 liters (26 gallons). The cylindrically shaped reactor vessel is 36 inches high and 18 inches in diameter with a maximum capacity of 40 gallons. The bottom of the vessel slopes to a 2 -inch Nominal Pipe Thread (NPT) exit nipple. The vessel is supported in a frame 60 inches high with a 32 -inch square top and bottom. The frame is made from 3 -inch square tubing with a wall thickness of $1 / 8$ inch. The support structure and vessel are designed so that if more volume is desired, a larger vessel (350-liter maximum) can fit directly into the frame. The frame is supported on four 6-inch diameter 
composite wheels so the system can be rolled to a suitable position.

The reactor is equipped with several components and features to aid in its operation. Figure 1.7 shows the top view of the reactor vessel. The top of the vessel is sealed with a $1 / 4$ inch steel plate fitted as a flange. This top plate, or lid, is equipped with 6 ports. One of the ports is located in the center of the top plate and houses a shaft that extends down into the vessel. There are two impellers attached to the shaft. The top impeller directs the flow from the top of the vessel downward and the bottom impeller directs the flow upward from the bottom of the vessel. This facilitates turbulent flow and provides good mixing. The shaft is driven by a 1 horsepower electric motor.

Two of the six ports are used to insert thermocouples in the reactor for temperature control and readout. The top plate is also is equipped with 2 access ports. One of the

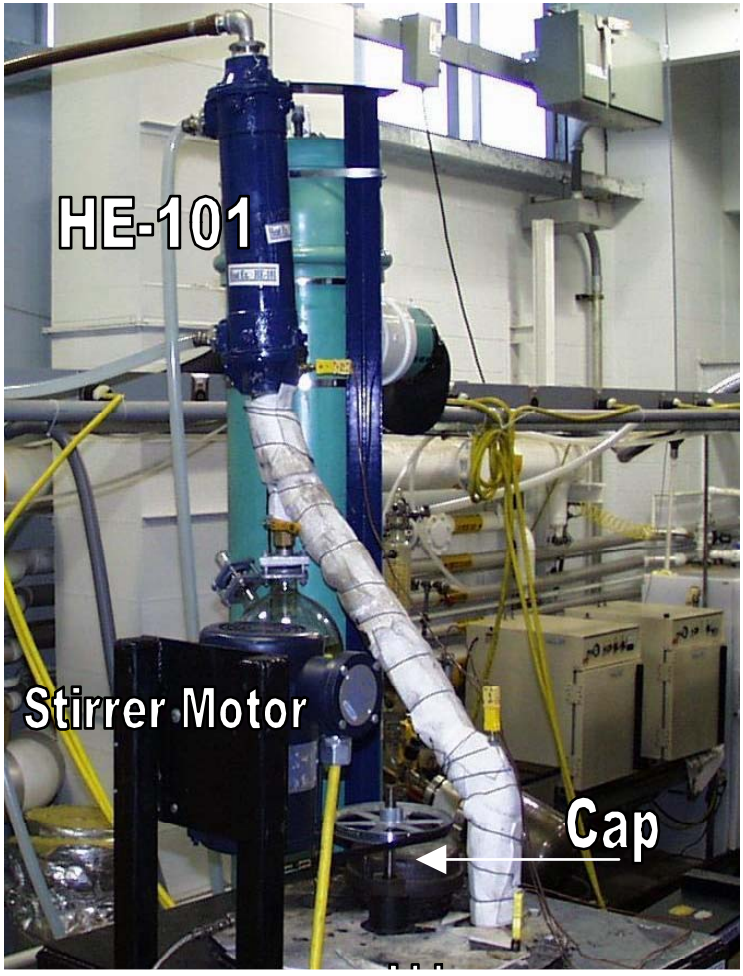

Figure 1.7 CSTR Lid Showing Motor, Cap, and Condenser access ports is a 6-inch threaded nipple welded into the lid. This nipple is equipped with a cap that can easily be removed to add fresh coal and solvent at the start of a run.

The other port is a 1-inch collar. Attached to the collar is a water-cooled heat exchanger (HE-101). This heat exchanger serves as a condensing unit to condense and collect the water that is driven off the NMP/coal slurry during the heating process. Water inhibits the solubility of coal in NMP and is accumulated in the system from the fresh coal or NMP coming into contact with air. As little as 3-mole \% water will significantly reduce the solubility of coal in NMP. The NMP/water vapors given off during the heating process are condensed and collected in a flask shown in Figure 1.6. The condensed vapors are collected until the coal/NMP slurry reaches its boiling point of $202^{\circ} \mathrm{C}$. At this point the mixture contains $\leq 0.4$ mole $\%$ water. Once the coal/NMP slurry reaches the boiling point, a valve is switched to allow the condensate from the condenser to flow back into the reactor. This solution in the overhead flask is stored until it can be distilled to remove the water from the NMP. The NMP is then recycled to the reactor for another run.

The reactor is kept under a nitrogen blanket to ensure that the NMP does not degrade under the elevated temperature. Opposite the side of the condenser is a $1 / 4$-inch compression fitting. Attached to this fitting is a gas line connected to a nitrogen cylinder. This enables the unit to be maintained under a nitrogen blanket. This will ensure that the solvent does not oxidize at the elevated operating temperature.

A normal run consists of 100 liters of NMP mixed with $10 \mathrm{~kg}$ of fresh coal ground to $\leq 60$ 
mesh $(0.25 \mathrm{~mm})$. The initial slurry fills about $70 \%$ of the total vessel volume; this allows for swelling of the coal particles and the thermal expansion of NMP. The reactor vessel is wrapped with 26 rope heaters. Each rope heater has a capacity of $500 \mathrm{~W}$ yielding a combined $13 \mathrm{~kW}$ heater that draws about $60 \mathrm{amps}$ at 220 volts. The coal/NMP takes approximately 2 hours to reach the boiling point of $202^{\circ} \mathrm{C}$. The mixture is kept at the boiling point for 1 hour.

The slurry must be cooled prior to centrifugation so as not to exceed equipment operational tolerances. The mixture is cooled to $100^{\circ} \mathrm{C}$ using a single pass shell and tube heat exchanger (HE-104). A gear pump (P-101) purchased from F.T. Thomas pumps is used to circulate the mixture through the tube side of the heat exchanger to cool the mixture to the maximum operating temperature of the centrifuge. The same gear pump is used to transfer the cooled slurry to the centrifuge where solids (undissolved material or waste coal) are separated from the liquid (NMP and the dissolved coal). 


\subsubsection{Centrifuge (CT-101)}

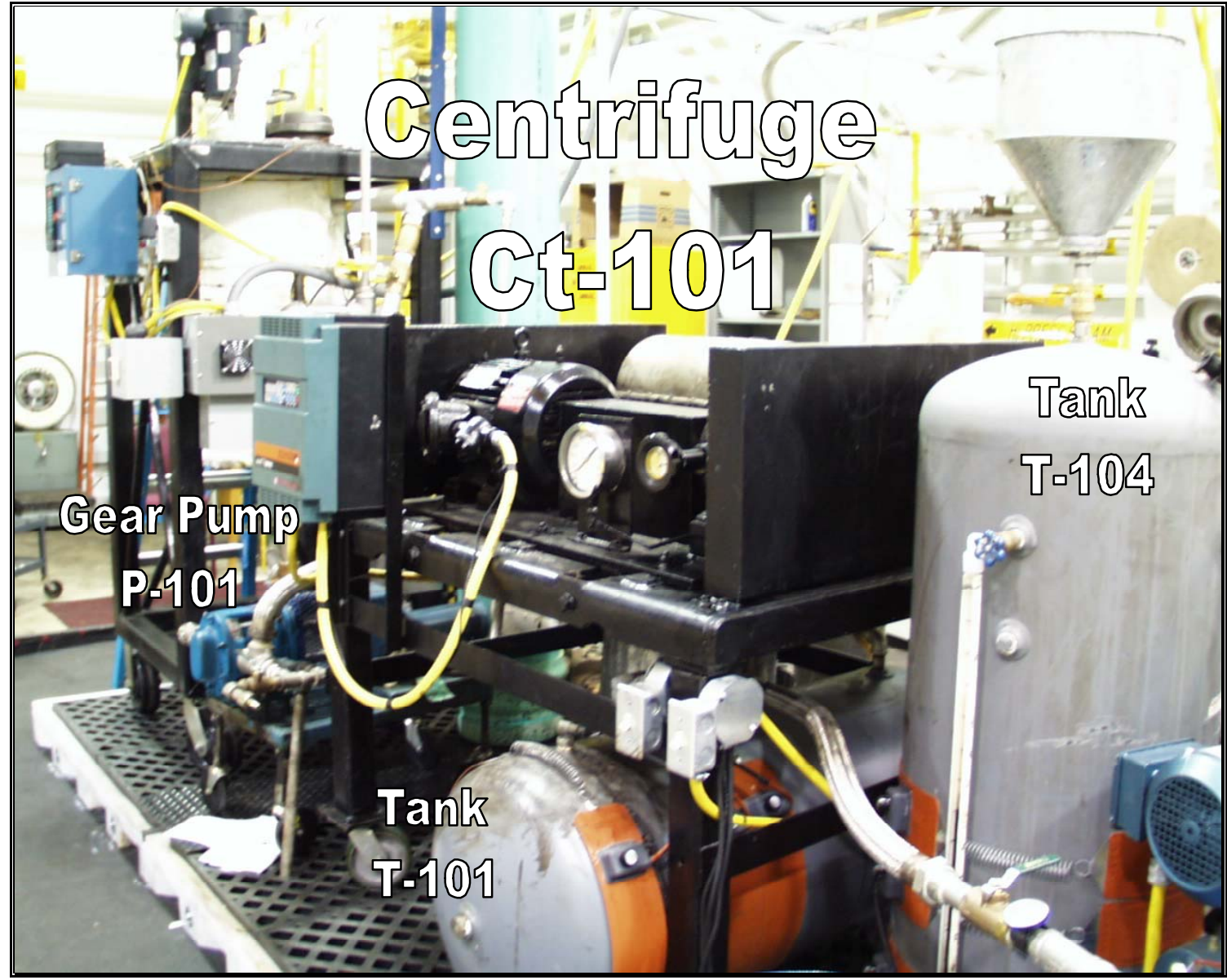

Figure 1.8 Sharples Penwalt BM-PF 290 Centrifuge

The centrifuge used in the pilot plant was donated to West Virginia University from Kopper's Corp. They previously used it to separate ash and carbon black from molten binder pitch. The unit is a Sharples Penwalt BM-PF 290 shown in Figure 1.8. The unit came without a controller. A suitable controller was purchased and integrated into the unit. Although the maximum RPM rating is 6000 , it was found that at 1458 RPM the unit achieved 2200 g's. Noting that this is $10 \%$ more than the previous centrifuge, it was felt that this was sufficient to guarantee the desired purity of the extract. The centrifuge has lowtemperature seals so the temperature of the input stream from the reactor must be cooled from $204^{\circ} \mathrm{C}$ to a maximum operating input temperature of $100^{\circ} \mathrm{C}$.

As shown in Figure 1.4, located between the CSTR and the centrifuge is a heat exchanger (HE-104). This heat exchanger was designed by us and made in our shop. It is a stainless steel shell and tube heat exchanger with an internal surface area of $7.3 \mathrm{ft}^{2}$ and an external surface area of $7.85 \mathrm{ft}^{2}$. A gear pump (P-101) is used to circulate the coal/NMP slurry from the reactor through the heat exchanger and back into the reactor until the temperature drops to $100^{\circ} \mathrm{C}$. It takes approximately $1 / 2$ hour of circulating the $\mathrm{NMP} /$ coal slurry through the heat exchanger to drop the reactor temperature from $204^{\circ} \mathrm{C}$ to $100^{\circ} \mathrm{C}$. The heat exchanger (HE- 
104) uses the exit water from the condenser (HE-101) -before it flows to the drain - to lower the temperature of the slurry.

Once the slurry has been cooled, it is fed to the centrifuge by the same pump (P-101). The flow rate into the centrifuge should not exceed 5 gallons per minute. This is a continuous centrifuge where the solid residue is extruded from one side and the filtrate flows from the other end. In this research, the solid residue contains the undissolved portion of the coal as well as ash material. The filtrate (dissolved coal and NMP) flows out the opposite end of the centrifuge and is collected in a 60-gallon tank (T-101). This tank is also equipped with heaters so the sample can be heated before it is sent to the wiped thin film evaporator. Tank T-101 also has an exit port so samples can be removed for analysis. If the filtrate contains too much ash, the sample can be pumped back into the CSTR and centrifuged again. This process could be repeated until the proper purity is obtained.

As mentioned previously, the solid residue or waste coal contains an appreciable quantity of NMP. NMP vapors are eliminated from the work area by placing a shroud over the residue collection bucket under the centrifuge. The shroud has an elephant trunk that draws the vapors toward a ventilation fan, exhausting the air from the building.

Between the shroud and the ventilation fan is an absorbing tower (ABS-101). The absorbing tower is 11 feet high. It is constructed from a piece of 12-inch SDR-35 PVC pipe. Jaeger Cascade Mini-Rings fill $6.5 \mathrm{ft}$ of the tower. The input vapor stream exits the shroud and enters the bottom of the absorber just below the ring support. The vapors are pulled up through 6.5 feet of water-saturated rings by the exhaust fan. At the bottom of the tower is a 15-gallon collection basin filled with water. A centrifugal pump (P-105) circulates water to the top of the absorber. Just below the gas exit port, the water is sprayed through a shower nozzle down through the rings. The water spray wets the plastic hoops and flows downward counter-current to the vapor stream. As the vapors leave the shroud, it passes over the wetted hoops and the NMP vapors are absorbed. Therefore, no NMP vapors are released to the work area or the environment.

Once the filtrate meets the desired purity (i.e. ash free) the filtrate is transferred to an upright tank (T-104) by a gear pump (P-102) purchased from F.T. Thomas Pump. This containment vessel is a 60-gallon compressed air tank that is vented to the scrubber. It is also equipped with a sight-glass so the level of the filtrate can be observed. Due to the fact that the tank (T-101) below the centrifuge it is in a horizontal position, accurate volume measurements were hard to achieve. The addition of the vertical tank (T-104) makes for more accurate evaluation of the fluid volume. This makes it possible to measure the filtrate volume and predict any solvent loss.

It is important to keep track of the solvent. The solvent can be four places after the centrifuge: (1) in the filtrate (NMP/dissolved coal) tank (T-101) under the centrifuge, (2) in the condenser collection flask above the reactor (HE-101), (3) in the waste coal, or (4) in the water of the absorber. The residue, or waste coal, leaving the centrifuge is wetted by the NMP so it is anticipated that some NMP would be lost with this residue. A good 
approximation of the solvent (NMP) losses could be made when the volume of the filtrate is measured in the vertical tank (T-104). Tank T-104 is calibrated using water and gradations are put on a sight glass. Filtrate from the horizontal vessel under the centrifuge is pumped into the vertical vessel and its volume is recorded. The vertical vessel also serves as a storage vessel so more than one run of the reactor can be done in a workday.

The NMP concentration in the absorber is only a trace. It would take several months to acquire appreciable amount. Once an appreciable amount of NMP is contained in the water, it will be removed and the water distilled out.

The NMP in the condenser above the reactor (containing water and NMP distilled off the slurry) will be distilled out and reused. The NMP entrained in the waste coal will be recovered by drying the residue in drying ovens (explained later). The NMP in the filtrate will be driven off, condensed, and recycled in the next three steps. Notice that the NMP is always recycled to be used again.

\subsubsection{Wiped Thin Film Evaporator}

The filtrate solution leaving the centrifuge is a mixture of a large quantity of NMP and a smaller quantity of dissolved coal. In order to process the coal, the volume must be significantly reduced. This is done through the use of a wiped thin film evaporator (TFE101) shown in Figure 1.9. The TFE is essentially a heated cylinder on to which the filtrate is sprayed. The TFE is used to remove most of the solvent by evaporation. The fluid filtrate (NMP and dissolved coal) is pumped into the head of the evaporation unit by use of a gear pump (P-104). A distribution plate sprays the fluid onto the heated walls of the cylindrical vessel. The heating unit (HE-102) uses Marlotherm HT as the heating media (fluid) to transfer heat to the cylinder wall at a specified temperature. The filtrate is wiped around the cylindrical wall by four blades attached to a rotor that turns on the central axis. The rotation rate of the wipers can be controlled. The blades are notched so that the less viscous fluid is lifted upward and the more viscous fluid is allowed to flow down to the exit port. The viscosity of the exiting fluid depends on the feed rate and filtrate temperature, wall temperature, operating pressure, and blade rotation rate. 

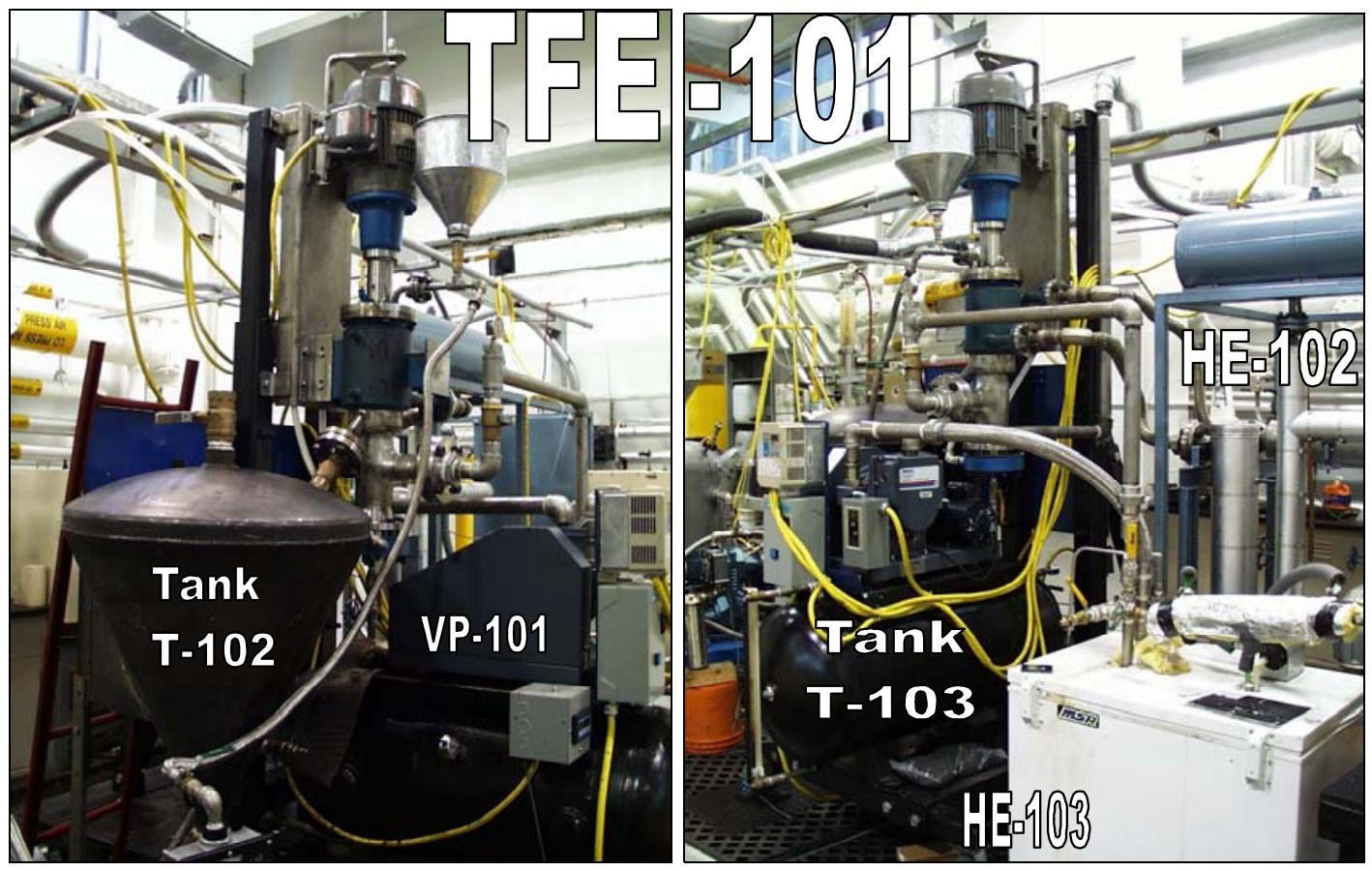

Figure 1.9 Pfaudler 1.2 $\mathrm{ft}^{2}$ Wiped Thin Film Evaporator

As the solvent evaporates from the heated surfaces, the wiper blades scrape the thickened residue away from the hot surface to an exit port. The exit port is affixed to a collection vessel (tank T-102) from which the sludge-like residue can be extracted and placed in the rotary evaporator $(\mathrm{RV}-101)$ for further drying. Tank $\mathrm{T}-102$ is cone shaped to facilitate the removal of the thickened extract.

The rotor in the TFE not only rotates the distribution plate and wiper blades, but also a "squirrel cage" fan. The rotation of the fan draws the vaporized solvent from the heated cylindrical surface to the center of the cylinder where a "cold finger" condenser condenses the solvent. An external water supply is used as the cooling source to chill the cold finger condenser. The solvent is condensed on the condenser and gravity fed into a containment vessel (tank T-103) below the TFE.

The entire evaporation system is under a vacuum and the pressure is externally controlled by vacuum pump VP-101. The system pressure controls the temperature at which the solvent evaporates. The solvent collection vessel (tank T-103) contains pure NMP and is equipped with a pump (P-103) that transports the NMP back to the CSTR for reuse. A large portion of the solvent is evaporated in the TFE and the concentration of the solids in the film is increased.

The TFE unit is actually three separate components working together: (1) the 
evaporator/wiper/separator (TFE-101), (2) the supportive heating unit (HE-103), and (3) the supportive vacuum pump (VP-101). The heating unit was purchased as a self-standing skid unit. Heat Exchanger HE-102 uses Marlotherm HT as the heat transfer oil with an upper operating temperature limit of $320^{\circ} \mathrm{C}$. HE-102 heats the oil to the desired operating temperature of the TFE. The hot oil is circulated over the external surface of the TFE cylinder wall supplying the heat needed to evaporate the NMP. The unit was sized properly and was equipped with a sensitive controller so the oil temperature can be kept essentially constant.

There are 5 operating conditions that control the TFE operation:

1. The flow rate of filtrate to the TFE $\left(\mathrm{F}_{\text {in }}\right)$.

2. The temperature of the filtrate feed to the TFE $\left(T_{\text {in }}\right)$.

3. The temperature of the heating fluid from the supporting heat exchanger $\left(T_{m}\right)$.

4. The film thickness on the wall—controlled by the blades rotation rate (RPM).

5. The internal pressure controlling the evaporation temperature $\left(\mathrm{P}^{\mathrm{sat}}\right)$.

TFE Operational parameters were initially approximated using pure water as a test fluid. Subsequent experimental testing led to the following optimal operating conditions. The fluid viscosity set the rotation rate of the blades at $350 \pm 20 \mathrm{RPM}$. It was found that the most favorable heating fluid temperature $\left(\mathrm{T}_{\mathrm{m}}\right)$ is $208^{\circ} \mathrm{C}$ with a filtrate flow rate $\left(\mathrm{F}_{\mathrm{in}}\right)$ of 0.5 $1 / \min$ and an input temperature $\left(\mathrm{T}_{\text {in }}\right)$ of $100{ }^{\circ} \mathrm{C}$. The Welch vacuum pump is sufficient to evacuate the 60-gallon vessel and all parts of the TFE to the desire operating pressure $\left(\mathrm{P}^{\text {sat }}\right)$ of below $10 \mathrm{~mm} \mathrm{Hg}$ pressure. Approximately $70 \%$ of the original volume of NMP is driven off at these operating parameters. This seemed to be the desired amount. If too much NMP is driven off from the purified coal extract the TFE will gum up. This does not happen with hydrogenated coal extract, which has a softening point below the boiling point of NMP. All of the NMP can be driven off of hydrogenated coal extract.

Located in line between the TFE and VP-101 is a cold trap/condenser (HE-103). This is a stainless steel trap of about 18 liters in volume. It is suspended in a bath of antifreeze kept in a small freezer unit. The freezer keeps the antifreeze at less than $-15^{\circ} \mathrm{C}$. This prevents any of the NMP from migrating to the pump. The pump oil is changed at regular intervals to ensure limited contamination and continued proper operation.

Once the NMP concentration in the filtrate has been reduced, the distilled clean NMP that is collected below the TFE is pumped back into the measuring vessel. This permits a determination of the efficiency of the TFE and also permits an approximation of the quantity of NMP that remains with the coal. The thickened pitch (Figure 1.10) is extracted from the pitch-receiving vessel and weighed. This also helps to approximate the amount of NMP remaining with the thickened extract. 


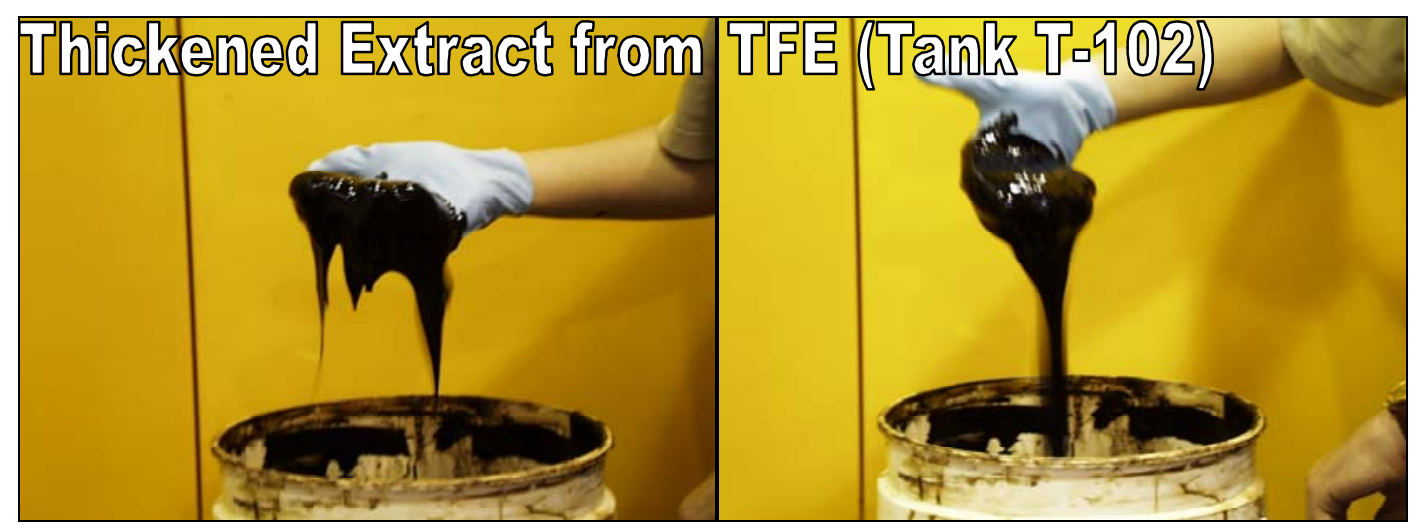

Figure 1.10 Thickened Extract Leaving Wiped Thin Film Evaporator

\subsubsection{Rotary Evaporator}
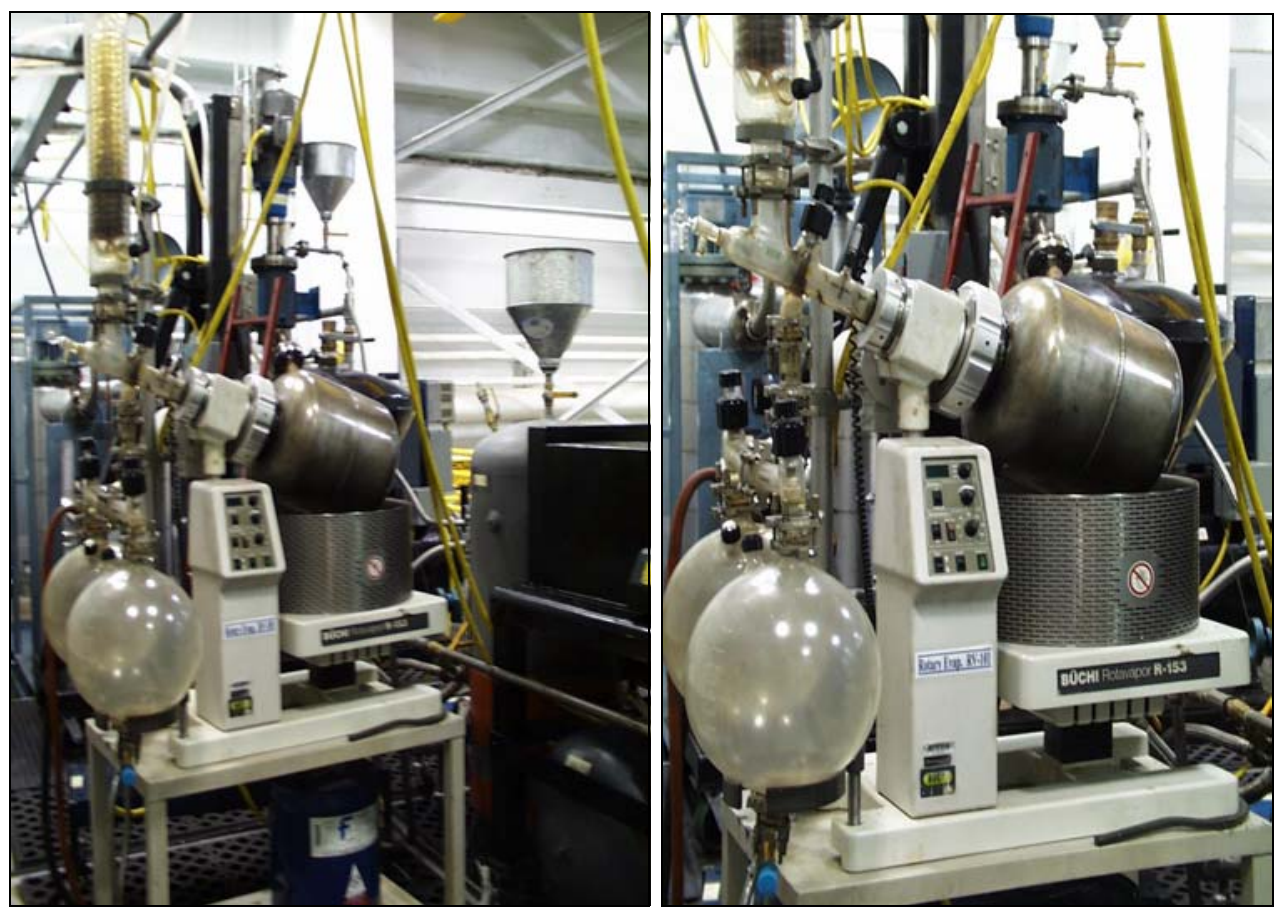

Figure 1.11 Modified Buchi R-135 Rotary Evaporator with Steel Tank

The next step in processing the extract is to further remove NMP from the thickened product. This is done using a rotary evaporation unit (Rotovap) shown in Figures 1.11. The Rotovap unit (RV-101) is essentially a thin film evaporator. It is composed of three parts: (1) the rotary vessel that contains the thickened extract from the TFE, (2) a condenser, and (3) a receiving vessel where the condensed NMP will be collected for recycle. The rotating vessel 
is a modified steel Freon bottle.

The steel bottle has a larger volume that the standard 10-liter glass, round bottom flask. Further, the steel bottle cannot be broken as easily. The steel bottle is partially filled with the thickened extract. About 3 gallons of material constitutes a batch. The steel bottle is attached to the Rotovap by a rotating flange. Once attached, the steel bottle is lowered into a hot oil bath and the system is evacuated to below $10 \mathrm{mmHg}$ using vacuum pump P-102. The condenser is then chilled using cold water. As the steel bottle is rotated, the thickened extract forms a thin film over the internal surface of the bottle. On the heated surface of the steel bottle the NMP is evaporated. The NMP vapors are then drawn by the Welch vacuum pump (VP-102) into the condenser where they are condensed to a liquid and collected in the glass bulbs for reuse.

As the solvent is removed from the thickened extract, the solid coalesces into large agglomerates. These have the appearance of small footballs or large softball. This unbalances the rotating vessel and the process is discontinued. This material is then removed, ground, and placed in trays and put in the drying ovens for the final drying.

\subsubsection{Vacuum Drying Ovens}

The Rotovap process removes the solvent to less than $50 \%$ by weight. The remaining $\mathrm{NMP} /$ dissolved coal mixture is a hard, solid mass. The remaining solvent can be removed to below the limits of detectability by vacuum drying. In this operation, the semi-dried, thickened extract is ground and spread out in stainless steel trays. The trays are then placed in vacuum drying ovens as shown in Figure 1.12. Each oven holds three trays and each tray holds about $3 \mathrm{~kg}$ of semi-dry, thickened extract. The purpose of the trays and grinding is to expose as much surface as possible.

The ovens are heated to $205^{\circ} \mathrm{C}$ under a $10 \mathrm{~mm} \mathrm{Hg}$ vacuum. The vapors are drawn through a condenser (HE-105), and chilled to below $-15^{\circ} \mathrm{C}$ by circulating antifreeze from the sump (reservoir) located below tank T-106 as seen in Figure 1.13. The NMP condensate is collected in tank T-106 and recycled for re-use. A small nitrogen flow is permitted to pass through the ovens to draw the NMP vapors to the condenser by the vacuum pump (VP-102). 


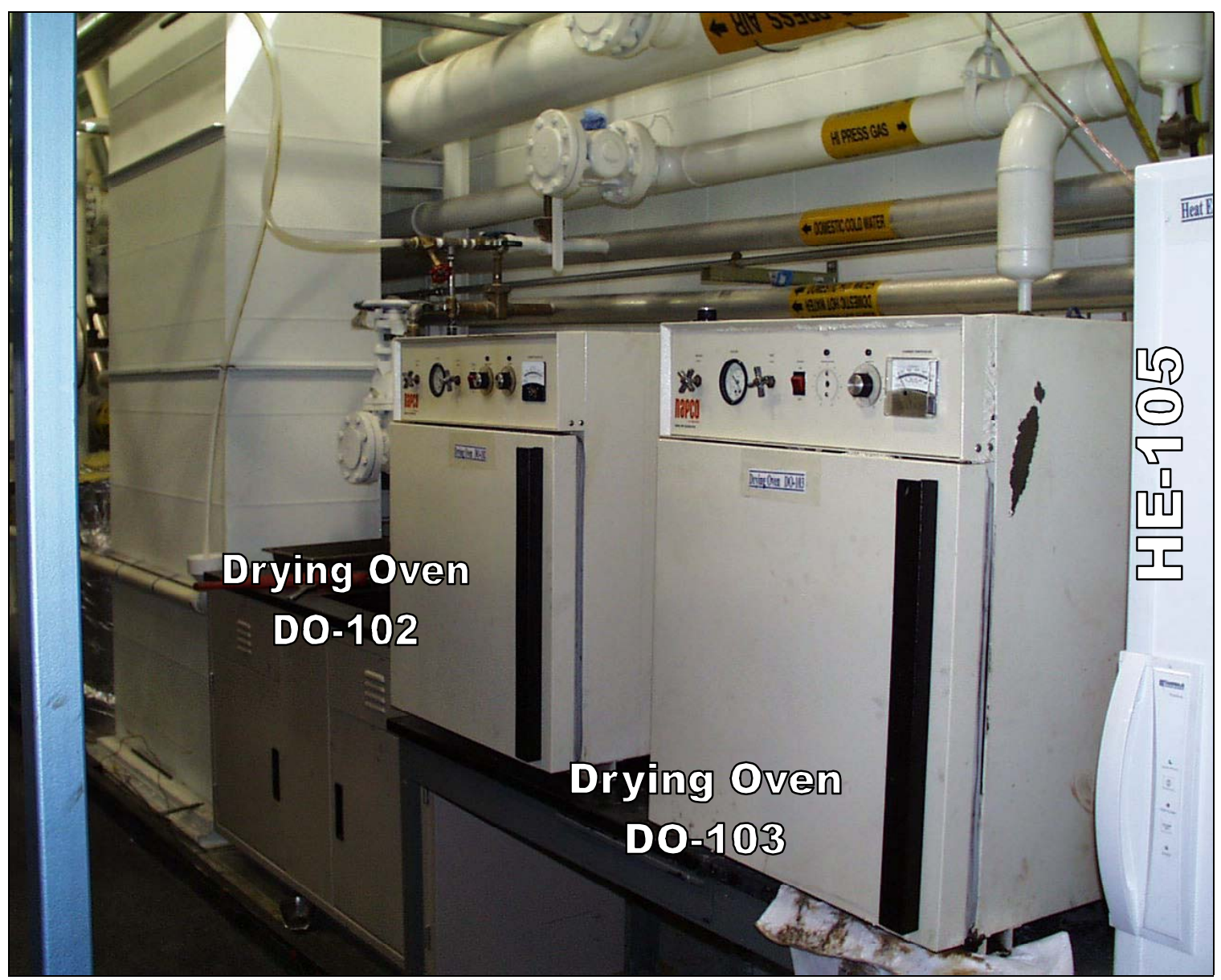

Figure 1.12 Processed Coal and Pitch Drying Ovens and Heat Exchanger

Figure 1.13 Drying Oven Condenser (HE105) and Collection Tank (T-106)

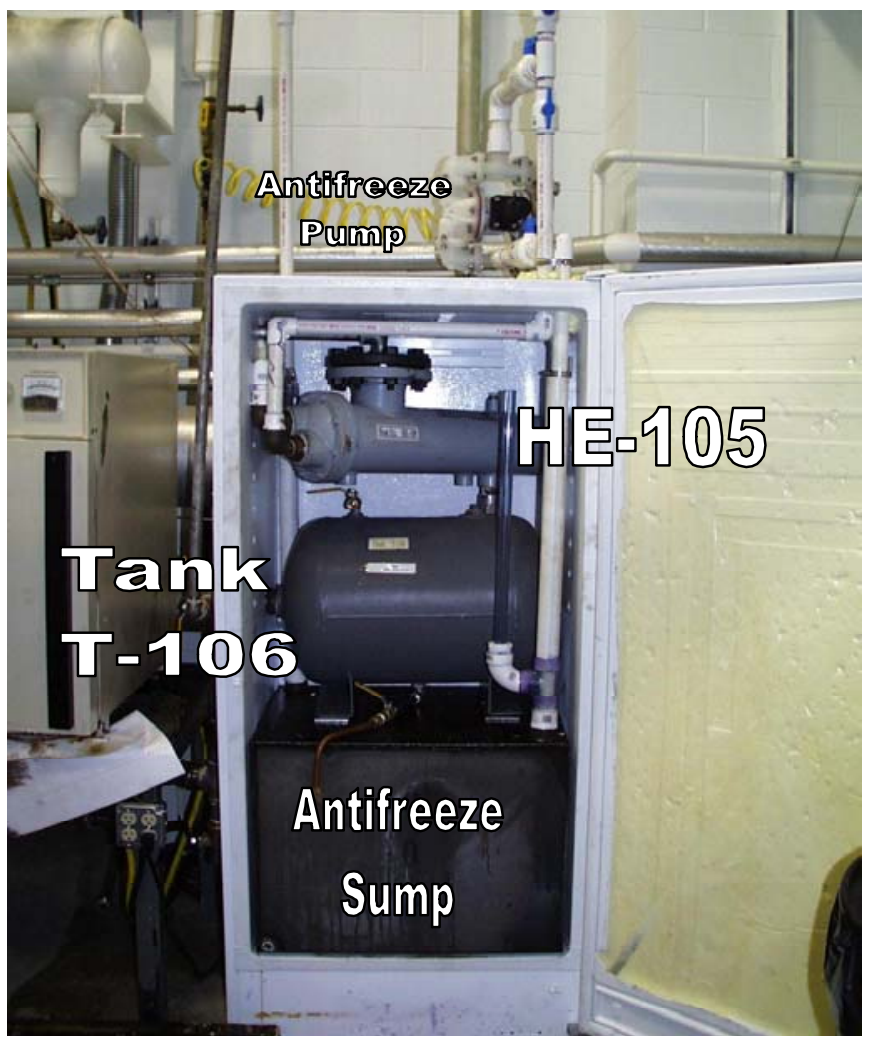


As the semi-dry thickened extract is dried, it is stirred so that more surface area is exposed. This process is repeated until no more fumes are detected at the condenser; at this time it is assumed that the coal extract is free of solvent. TGA analysis of the solid showed no weight loss at $205^{\circ} \mathrm{C}$. This indicated that indeed all the solvent has been removed

\section{$1.5 \quad$ Results}

\subsubsection{Material Balance}

One of the major goals of this research project was to perform a material balance over the CEPU to determine the actual extract yield, solvent loss, and percent mass balance closure (\%MBC) on the weight of the raw coal, extract and residue. The amount of solvent loss is a major concern. Loosing a small percentage of the original solvent would greatly affect the cost of the coal-based carbon pitch produced. Under normal operation conditions the solvent can leave the system in only three ways: as residual solvent in the dried pitch, as solvent not recovered in the dried process coal waste (residue), and solvent loss cleaning up the solvent/water mixture taken off of the reactor by condenser (HE-101) as explained earlier.

Using the ultimate analysis preformed on the coal, centrifuged processed coal, dried centrifuged processed coal, and the dried extract or "pitch", a material balance on the solvent can be done over the entire system. The overall solvent material balance for this research neglected the solvent that might be lost in cleaning up the solvent/water mixture from the reactor's condenser (HE-101). The amount of solvent recovered from the solvent/water mixture would be a function of how much water the coal contained, whether or not the coal was dried, and what type of clean up method was used.

The research presented here focused on the amount of residual or solvent retention in the extract and residue. Currently there is no quantitative measurement of the solvent retention, however, an apparent solvent retention level can be calculated using the ultimate analysis of the raw coal, extract and residue. The major assumption in this calculation is that all increased nitrogen in the extract and residue originates from the nitrogen in NMP. It may however, originate from the nitrogen in the air or the nitrogen that is used to purge the extraction vessel and the vacuum ovens (Gerstner 1989). Also, the absolute amount of nitrogen in the raw coal, extract, and residue is small. This smaller number can lead to large errors in the determination of the amount of increased nitrogen. Therefore, the results are considered to be an inflated value for the level of solvent retention in the extract and can be assumed to be the worst-case scenario. 


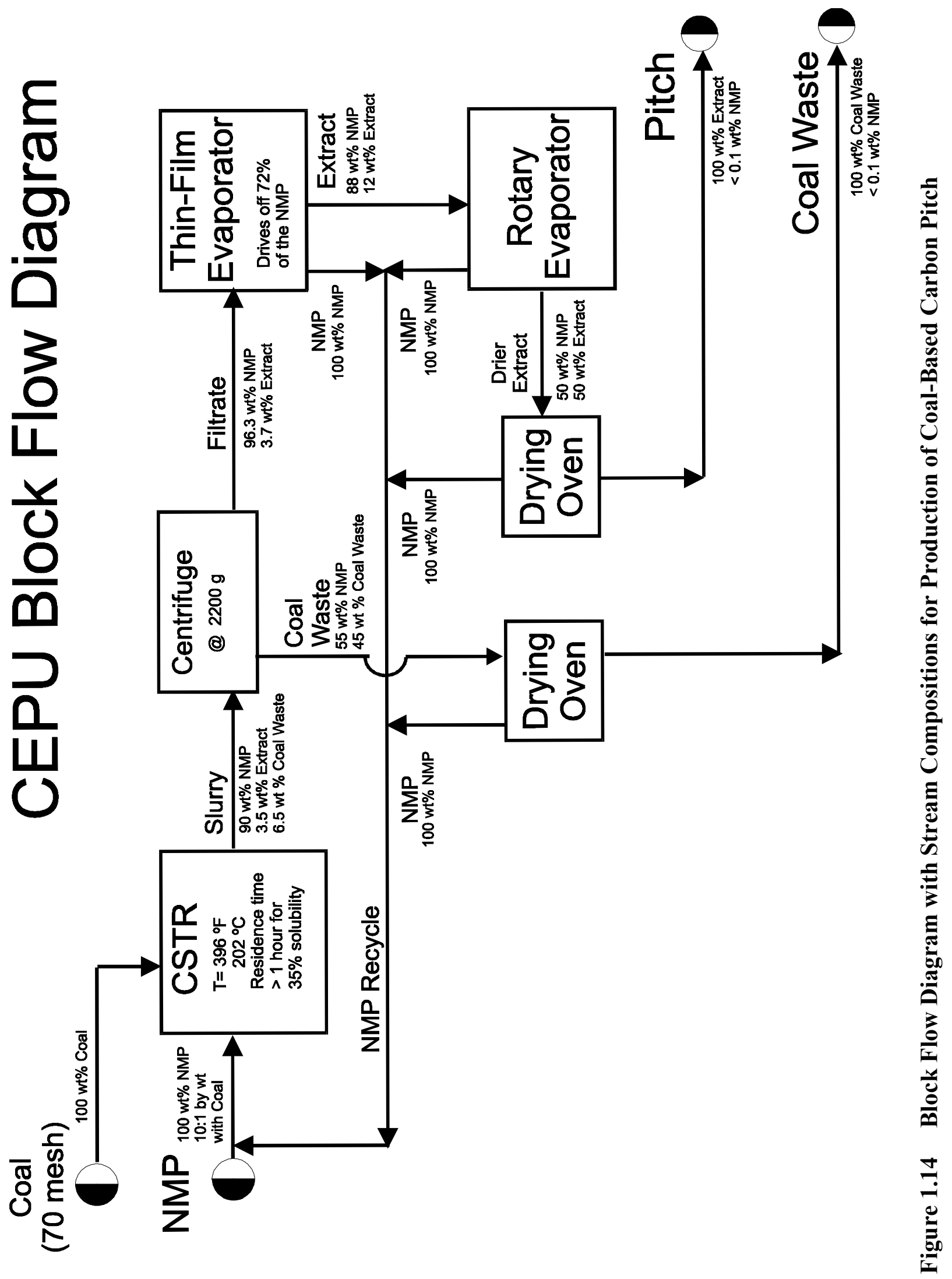




\subsubsection{Overall Material Balance}

A few terms need to be defined to calculate the overall material balance for the coal and solvent over the CEPU.

First, the $\%$ yield, or percent extraction is the percentage of the feed coal that is dissolved or extracted. The \% yield is calculated using the Equation 1.1 below.

$$
\% \text { yield }=\frac{\text { (wt. of raw coal }- \text { wt. or residue) }}{\text { wt. of raw coal }} * 100
$$

This is the most practical method, in determining how well the coal was extracted by the NMP. However, it is not the most accurate due to the fact that it considers the ash or moisture content of the raw coal that are not extractable.

The Moisture and Ash Free percent yield calculation (\% yield MAF) does not take into account the portion of the raw coal that is not extractable material, namely the water and ash. So the amount of water and ash is subtracted out of the weight of the coal to determine the amount of organic material that is extractable. This is a more accurate and consistent basis of which to compare the performance of various coals in extraction procedure (Gerstner 1989). For this calculation, the extract is assumed to be essentially dry and ash-free which is confirmed by the proximate analysis. The \% yield (MAF) is calculated using Equation 1.2 shown below.

$$
\begin{aligned}
& \% \text { yield }(\mathrm{MAF})=\frac{\mathrm{wt} . \text { of extract }}{(1-\mathrm{A} \%-\mathrm{M} \%) * \text { wt. of raw coal }} * 100 \\
& \text { where: } \\
& \quad \mathrm{A}=\text { Ash from proximate analysis of raw coal } \\
& \mathrm{M}=\text { Moisture from proximate analysis of raw coal }
\end{aligned}
$$

Percent Mass Balance Closure (\% MBC) is calculated using Equation 1.3. This is an indication of the operational performance of the CEPU by weight o raw coal. A \% MBC greater that $2 \%$ indicates that there is significant solvent retention. $\mathrm{A} \% \mathrm{MBC}$ less that -2 $\%$ indicates that there is a problem somewhere in the system.

$$
\% \mathrm{MBC}=\frac{\text { (wt. of extract }+ \text { wt. of residue) }- \text { wt. of raw coal }}{\text { wt. of raw coal }} * 100
$$


The apparent percent nitrogen retention is back calculated by determining the increase in nitrogen content of the extract and the residue as compared to the raw coal as follows:

$$
\begin{gathered}
\text { increased } \mathrm{N}=\left(\frac{\% \mathrm{~N}_{\mathrm{R}}}{100} * \text { wt. of residue }+\frac{\% \mathrm{~N}_{\mathrm{E}}}{100} * \text { wt. of extract }\right)- \\
\frac{\% \mathrm{~N}_{\text {Raw }}}{100} * \text { wt. of raw coal }
\end{gathered}
$$

$$
\begin{array}{ll}
\text { where: } & \mathrm{N}_{\mathrm{R}}=\% \text { Nitrogen of Residue }^{\mathrm{a}} \\
& \mathrm{N}_{\mathrm{E}}=\% \text { Nitrogen of Extract }^{\mathrm{a}} \\
& \mathrm{N}_{\text {Raw }}=\% \text { Nitrogen of Raw Coal }{ }^{\mathrm{a}} \\
& { }^{\mathrm{a}} \text { From Ultimate Analysis Wet Basis }
\end{array}
$$

The apparent level of solvent retention can then be calculated by relating the increased nitrogen content to the mass ratio of nitrogen in NMP in the following manner:

$$
\% \text { Solvent Retention }=\frac{\text { increased } \mathrm{N} * \frac{\mathrm{MW}_{\mathrm{NMP}}}{\mathrm{MW}_{\mathrm{N}}}}{\text { wt. or raw coal }} * 100
$$

where: $\quad \mathrm{MW}_{\mathrm{NMP}}=99.1$

$$
\mathrm{MW}_{\mathrm{N}}=14
$$

Using the Ultimate Analysis, an overall material balance can be done over the system. The results for the material balance are shown in Table 1.5.

\subsubsection{Material Balance Over Individual Units}

Now that the overall material balance for the system had been considered, the material balances over the five main components of the system as seen on the Block Flow Diagram in Figure 1.14 will be considered.

- Continuous Stirred Tank Reactor (CSTR)

- Centrifuge

- Wiped Thin Film Evaporator (TFE) 
- Rotary Evaporator (Rotovap)

(RV-101)

- Drying Ovens

(DO-101-3)

This material balance is important for calculating the energy balance over the system next.

\subsubsection{Material Balance Over Continuous Stirred Tank Reactor (CSTR)}

(R-101)

Again the CSTR (reactor) is loaded with a 10:1 ratio by weight of NMP to coal. It is heated to $202^{\circ} \mathrm{C}$ while stirring. The reactor is then kept at $202^{\circ} \mathrm{C}$ for one hour. NMP dissolves the Upper Powleton coal giving the yield of $36 \pm 2 \%$ and a yield (MAF) of $39 \pm 2 \%$ shown for the overall material balance. The slurry of dissolved coal in NMP and undissolved coal is then fed to the Centrifuge (Ct-101).

The fresh coal used in the CEPU is not dried prior to processing. Therefore the reactor is designed so that the water can be driven off, condensed, and cleaned up. Again, the overall material balance for this research neglected the solvent that might be lost in cleaning up the solvent/water mixture from the reactor's condenser (HE-101). The amount of solvent recovered from this would be a function of how much water the coal contained, whether or not the coal was dried, and what type of clean up method was used.

The average amount of solvent driven of by the reactor appeared to be 1 to 2 liters. This volume of solvent loss was not thought to be enough to affect the \% yield. The condensed NMP is collected until there was enough to load the reactor with an appreciable quantity. The reactor then serves as a distillation column by heating several batch loads to the boiling point $\left(202^{\circ} \mathrm{C}\right)$ of NMP. Once at $202^{\circ} \mathrm{C}$ the essentially pure NMP solution is cooled and pumped into the upright tank (T-104) to be used in the next run. The water and NMP vapors that are driven off are condensed and collected. The reactor is always operated at atmospheric pressure. Therefore, using the glass collection flask affixed to the bottom of the condenser (HE-101), the boiling temperature is noted. This boiling temperature is used to calculate the amount of water in mixture using the vapor/liquid equilibrium diagram. The procedure listed above is repeated until the mixture contains only 40 -mole $\%$ NMP.

\subsubsection{Material Balance Over Centrifuge $\quad($ Ct-101)}

Again, the centrifuge is fed the slurry of dissolved coal in NMP and undissolved coal from the reactor by pump P-101 at 2 liters/min. The feed is approximately $92 \%$ NMP $3 \%$ Dissolved coal (extract), and $5 \%$ undissolved coal by weight. The centrifuge, operating at $2200 \mathrm{~g}$, separates the solution of dissolved coal and NMP from the solid undissolved coal.

The dissolved coal in NMP leaves the centrifuge and is feed to the TFE where a major portion of the NMP is then driven off. The TFE feed stream contains approximately 96.3 weight $\%$ NMP and 3.7 weight $\%$ extract. 
The undissolved coal leaves as a moist granular solid containing $55 \pm 2$ weight $\%$ NMP. This solid resembles damp potting soil. NMP is still entrapped in the undissolved coal particles, yet the particles are not saturated. The undissolved coal is then placed in a vacuum drying oven where the NMP is driven off.

\subsubsection{Material Balance Over Wiped Thin Film Evaporator (TFE) (TFE-101)}

The TFE is utilized to drive off a major proportion of the NMP from the extract. The TFE drives off $72 \pm 2 \%$ of the NMP in the feed stream from the centrifuge using the $20 \mathrm{~kW}$ supporting heat exchanger. The evaporated NMP off is condensed and recycled for another run. The product of the TFE is a "thickened extract". This thickened extract, containing 89 $\%$ NMP by weight and $11 \%$ extract, is then feed to the Rotary Evaporator (Rotovap).

\subsubsection{Material Balance Over Rotary Evaporator (Rotovap) (RV-101)}

The thickened extract from the TFE is fed to the Rotovap in batch loads. The Rotovap uses a hot oil bath to drive off approximately $88 \%$ by weight of the NMP contained in the thickened extract. This is $24 \%$ by weight of the NMP in the filtrate from the centrifuge. The product leaving the rotovap is a semi-solid mass of $50 / 50 \%$ by weight extract/NMP. This solid is sent to a vacuum drying oven to complete the drying process.

Notice that the Rotovap drives off $1 / 3$ the amount of NMP in the filtrate as that evaporated in the TFE. Therefore, the addition of the TFE reduces the number of batches in the Rotovap by a factor 4 .

\subsubsection{Material Balance Over Drying Ovens $\quad$ (DO-101-3)}

The Drying Ovens are the final step in the process. The solid extract leaving the Rotovap and the residue leaving the centrifuge are sent to the Drying Ovens to remove the NMP down to less than $2 \mathrm{wt} \%$. Note that this is a calculated number from the Ultimate Analysis of the fresh coal, dried waste coal, and final dried coal-based carbon pitch.

Table 1.5 Material Balance Results

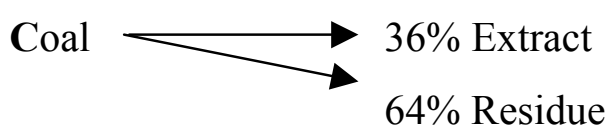




$\begin{array}{ll}\text { Yield } & 36 \pm 2 \% \\ \text { Yield (MAF) } & 39 \pm 2 \% \\ \text { MBC } & <1 \% \\ \text { Solvent Retention } & <0.7 \% \text { (by wt of Raw Coal) } \\ & <2 \% \quad \text { (by wt of Extract) }\end{array}$

$\begin{array}{llll} & \text { Fresh Coal } & \text { Extract } & \text { Residue } \\ & 100 \% & 36 \% & 64 \% \\ \text { \% Volatile Matter } & 26 & 30 & \\ \text { \% Fixed Carbon } & 64 & 68 & \\ \text { BTU/lb } & 14200 & 15000 & 13200 \\ \text { \% Carbon } & 84 & 87 & 77 \\ \text { \% Hydrogen } & 3.4 & 3.8 & 2.7 \\ \text { \% Nitrogen } & 0.6 & 1.2 & 0.47 \\ \text { \% Oxygen } & 4.1 & 5.2 & 3.0 \\ \text { \% Sulfur } & 0.8 & 0.7 & 0.7 \\ \text { \% Ash } & 6.8 \% & <0.6 \% & 8.5 \%\end{array}$

\subsection{Energy Balance}

An energy balance over the CEPU can be calculated once a detailed material balance for the CEPU is complete. The energy balance for this system focuses on the amount of thermal energy associated with the operation of four of the five main components and their supporting units (i.e. heat exchangers) of the CEPU. The Centrifuge is not mentioned in this section due to the fact that there is only mechanical work done by the centrifuge, no thermal. Thus the four main components are:

- Continuous Stirred Tank Reactor (CSTR)

$(\mathrm{R}-101)$

- Wiped Thin Film Evaporator (TFE)

(TFE-101)

- Rotary Evaporator (Rotovap)

(RV-101)

- Drying Ovens

(DO-101-3) 


\subsubsection{Thermal Properties of NMP}

The physical properties of NMP and coal are needed to calculate the energy balance. Most of NMP's physical properties can be found in Table 1.1. Listed below are the major thermal properties of NMP needed for the energy balance.

$$
\begin{aligned}
& \text { Specific Heat }\left(\mathrm{Cp}_{\mathrm{NMP}}\right): \quad \quad \quad 1.67 \mathrm{~J} / \mathrm{kg} /{ }^{\circ} \mathrm{C} \text { at } 20^{\circ} \mathrm{C} \\
& \mathrm{Cp}_{\mathrm{NMP}}=3.37 * 10^{\wedge}-3 *(\mathrm{~T})+1.59 \\
& \text { Thermal Conductivity }\left(\mathrm{k}_{\mathrm{NMP}}\right): \quad \quad \mathrm{k}_{\mathrm{NMP}}=-1^{*} 10^{\wedge}-4(\mathrm{~T})+0.1954 \\
& \left(\mathrm{~T}\left[{ }^{\circ} \mathrm{C}\right], \mathrm{k}_{\mathrm{NMP}}\left[\mathrm{W} / \mathrm{M}^{\circ} \mathrm{C}\right]\right)
\end{aligned}
$$

Even though NMP makes up the majority of the composition of the streams in the CEPU, its concentration drops as a batch progresses through the CEPU process. Therefore, some of the thermal properties of coal need to be defined.

\subsubsection{Thermal Properties of Coal}

The specific heat of coal is a thermal property needed to calculate the energy associated with heating and cooling the coal in the CEPU. As seen in Figure 1.15, the specific heat of coal increases with volatile matter and temperature (Volborth 1987). Figure 1.15 shows the specific heat of coal with $25 \%$ volatile matter at $200^{\circ} \mathrm{C}$ to be about $1.6 \mathrm{~kJ} / \mathrm{kg} \mathrm{K}$. The coal used in this research project contains less than $25 \%$ volatile matter. As a result, the research presented here assumes the energy need in heating and cooling of coal to be the same as that of NMP. NMP has a specific heat of $1.67 \mathrm{~J} / \mathrm{kg} /{ }^{\circ} \mathrm{C}$ at $20^{\circ} \mathrm{C}$ and increase with temperature. This is greater than that of coal at $202^{\circ} \mathrm{C}$ (the maximum operating temperature of the CEPU).

The assumption that coal has the same specific heat as NMP may lead to slightly inflated values for the energy balance. However, the numbers calculated here are theoretical values and do not take into account the equipment operating efficiency. Therefore, the inflated values are thought to be an order of magnitude less that the adjusted values for equipment efficiency. If one is so inclined, more detailed models for the specific heats of coals are presented by Kirov (1960) and van Krevelen (1961). 


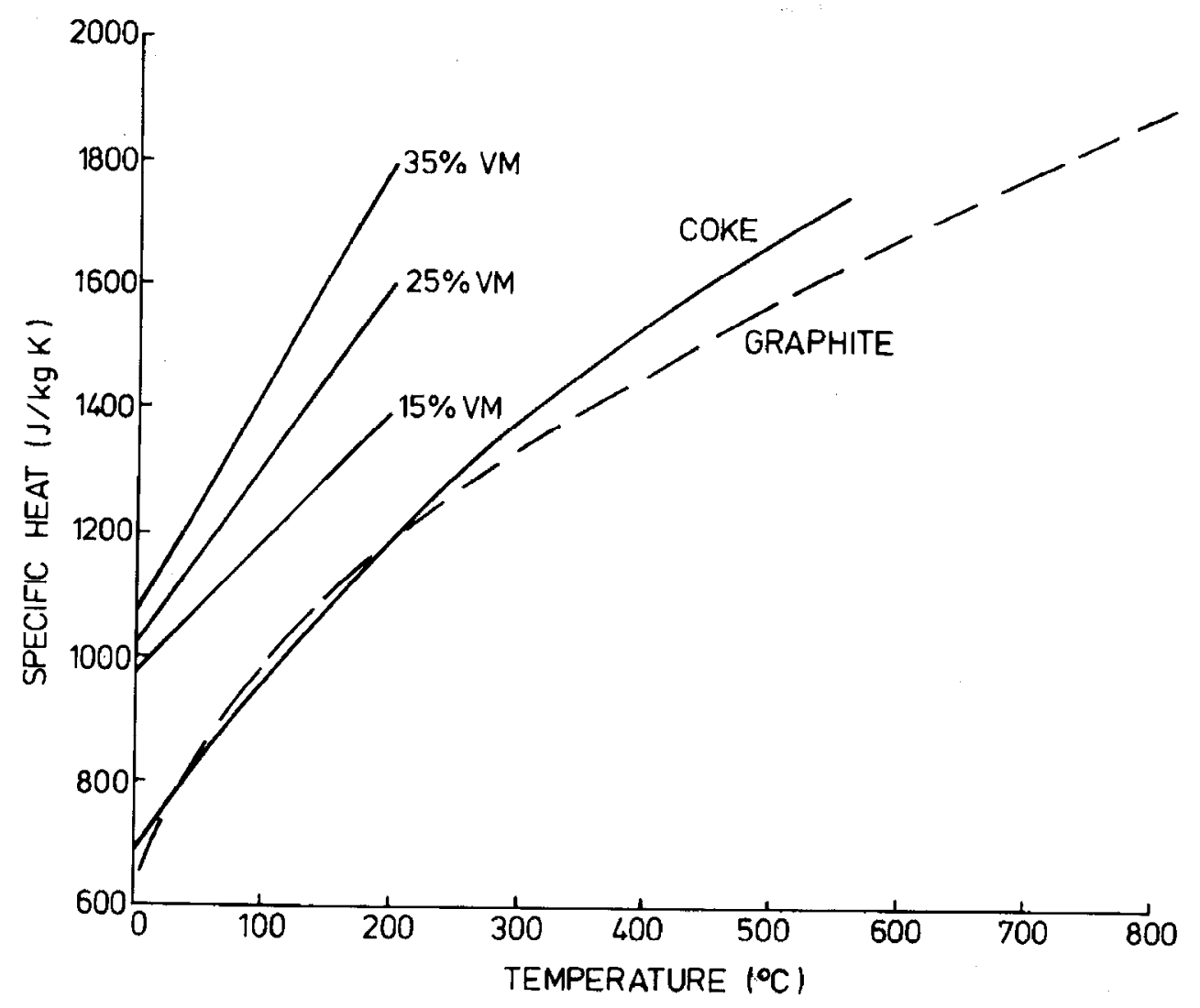

Figure 1.15 Typical Values of the Specific Heats of Coal, Coke and Graphite. (Volborth 1987)

The thermal conductivity of coal is the second property that is important for the energy balance on the Rotovap and drying ovens. The thermal conductivity of coal is calculated using Equation 1.8 (Volborth 1987).

$$
\frac{1}{\mathrm{k}_{\text {Coal }}}=\left(\frac{\mathrm{C}}{1.47}+\frac{\mathrm{H}}{.0118}\right)^{*}\left[\left(\frac{273}{\overline{\mathrm{T}}}\right)^{\wedge} 0.5\right]
$$

Where: $\mathrm{C}$ and $\mathrm{H}$ are mass fractions of Carbon and Hydrogen, and $\mathrm{T}$ is absolute temperature in $\mathrm{K}$.

At $0^{\circ} \mathrm{C}(\mathrm{T}=273 \mathrm{~K})$, the equation predicts the measured thermal conductivity of amorphous carbon $(1.47 \mathrm{~W} / \mathrm{m} \mathrm{K})$ for the limiting case of pure carbon $(\mathrm{C}=1)$. For a typical bituminous coal $(\mathrm{C}=0.85, \mathrm{H}=0.05)$ at $0^{\circ} \mathrm{C}$, the equation predicts a thermal conductivity of $0.21 \mathrm{~W} / \mathrm{m} \mathrm{K}$. This value lies within the range of literature data for monolithic specimens of bituminous coal (Volborth 1987). 


\subsubsection{Energy Balance on The Continuous Stirred Tank Reactor (CSTR) (R-101)}

Again, the CSTR (reactor) is used to heat the fresh coal/NMP solution from $25^{\circ} \mathrm{C}$ to $202^{\circ} \mathrm{C}$. Loaded with $100 \mathrm{~kg} \mathrm{NMP}$ and $10 \mathrm{~kg}$ coal, this temperature increase of $177^{\circ} \mathrm{C}$ requires 38.4$\mathrm{MJ}$ of thermal energy assuming the average specific heat of $1.97-\mathrm{kJ} / \mathrm{kg}{ }^{\circ} \mathrm{C}$ from Equation 1.6. Assuming that a max of 2 liters of NMP and water are driven off and condensed by condenser (HE-101), the extra energy needed is $1.1 \mathrm{MJ}$. This brings the total to $39.5 \mathrm{MJ}$ of energy needed to heat a batch.

The reactor was designed with a 26 rope heaters operating each at $500 \mathrm{~W}$, generating a total of $13 \mathrm{~kW}$. Experimental results have shown that it takes 2 hours to bring the mixture of NMP and coal to a boil. Figure 1.16 shows the heating rate for the CSTR (R-101) with $100 \mathrm{~kg}$ pure $\mathrm{H}_{2} \mathrm{O}, 100 \mathrm{~kg}$ pure NMP, and a batch of $100 \mathrm{~kg} \mathrm{NMP}$ and $10 \mathrm{~kg}$ of coal.

The heaters produce a total of 93.6 MJ of energy during the two hours of heating, assuming that the heaters all work at $100 \%$. This makes the reactor's heaters about $42 \%$ efficient in heating. This low efficiency may be due to some heaters not working, heating of the metal walls and piping of the reactor itself, heat loss by convection to the surroundings, to the room temperature $\mathrm{N}_{2}$ purge, and the vapors that are condensed and allowed to flow back into the reactor.

The reactor was cooled to calculate how much heat was actually lost by the reactor. The cooling temperature profile was plotted in Figure 1.17. From this figure the rate of heat loss by the reactor can be calculated. 
WVSC-001-DMF Swelling

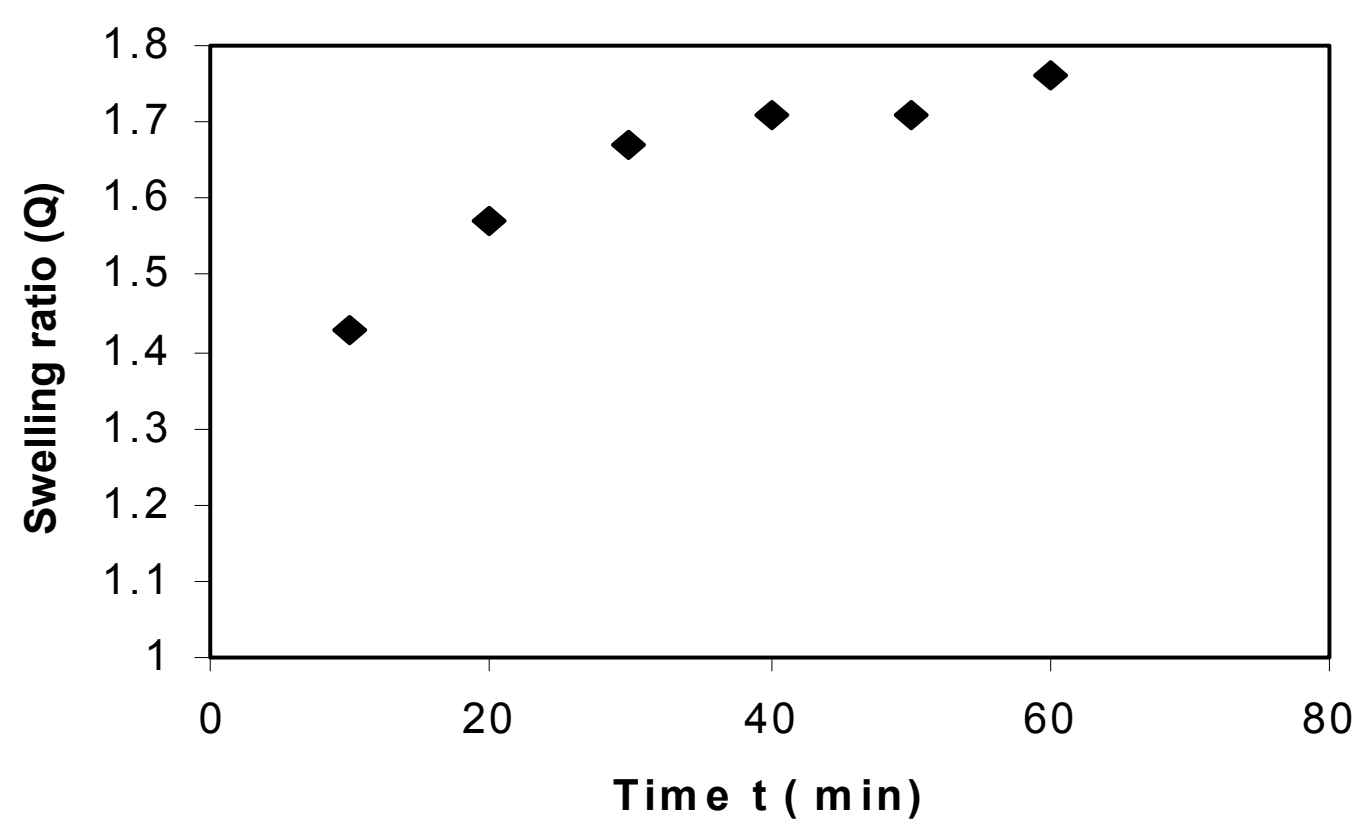

Figure 1.16 CSTR (R-101) Temperature Profile for Heating

Figure 1.17 shows the rate of heat loss of the reactor under three different conditions. Notice that the NMP solution cooled faster than the NMP/coal slurry even thought it was at a lower temperature. This is because the pure NMP solution was circulated through the un-insulated recycle pipes while it was cooling, and the NMP/coal slurry was not. The reactor cooled down $28^{\circ} \mathrm{C}$ in the time of 2 hours. Assuming that only the NMP/coal mixture was cooled, a calculated rate of $3.3 \mathrm{MJ}$ per hour was lost at an average rate off $0.92 \mathrm{~kW}$.

Along with the $13 \mathrm{~kW}$ rope heaters, the reactor has two supporting heat exchangers (HE-101 $\& 104)$. These heat exchangers use tap water as the cooling media. HE-101 condenses the vapors driven off from the reactor. HE-104 cools the NMP/coal slurry in the reactor before it is pumped to the centrifuge to stay within operational limits of the unit. 


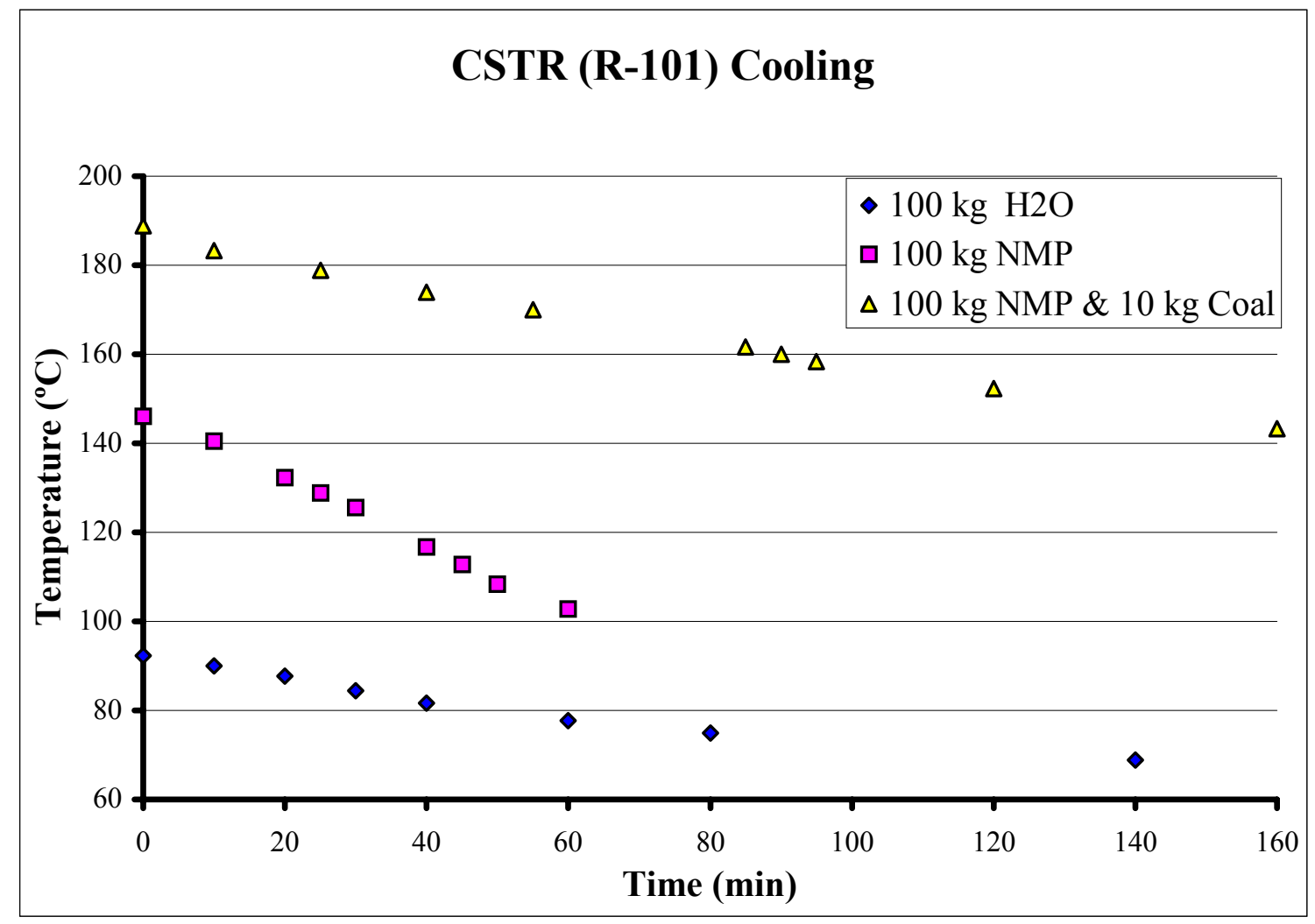

Figure 1.17 CSTR (R-101) Temperature Profile for Cooling the Reactor Without Supporting Heat Exchanger HE-104

\subsubsection{Reactor $\mathrm{NMP} / \mathrm{H}_{2} \mathrm{O}$ Vapor Condenser Heat Exchanger (HE-101)}

Heat Exchanger HE-101 uses tap water on the tube side as a cooling medium to condense NMP and water vapors boiled off of the NMP/coal slurry in the reactor on the shell side. This stainless steel heat exchanger was sized so that it could handle a thermal rate well above the maximum reactor output of $13 \mathrm{~kW}$. The reactor operating at $100 \%$ efficiency can vaporize a maximum rate $1.45 \mathrm{~kg} / \mathrm{min}$ of NMP.

The tube side of the stainless steel heat exchanger transfers heat by forced conduction of water, and the shell side of the heat transfer transfers heat by condensation of vapor. The temperate driving force from shell side to the tube side is more than $180^{\circ} \mathrm{C}$.

Using Equation 1.9 (Holman 1997), the overall heat-transfer coefficient $\left(\mathrm{U}_{\mathrm{o}}\right)$ for the shell side was calculated to be $1480 \mathrm{~W} / \mathrm{m}^{2}{ }^{\circ} \mathrm{C}$. The maximum rate of heat transfer $\left(\mathrm{Q}_{\max }\right)$ for this heat exchanger was calculated to be over $400 \mathrm{~kW}$ using Equation 1.0 (Holman 1997). 


$$
\mathrm{U}_{\mathrm{o}}=\frac{1}{\frac{\mathrm{r}_{\mathrm{o}}}{\mathrm{r}_{\mathrm{i}}} \frac{1}{\mathrm{~h}_{\mathrm{i}}}+\frac{\mathrm{r}_{\mathrm{o}}}{3.14} * \frac{\ln \frac{\mathrm{r}_{\mathrm{o}}}{\mathrm{r}_{\mathrm{i}}}}{\mathrm{k}_{\mathrm{ss}}}+\frac{1}{\mathrm{~h}_{\mathrm{o}}}}
$$

where: $r_{o}$ is the outside radius of the tubes,

$r_{i}$ is the inside radius of the tubes,

$\mathrm{h}_{\mathrm{o}}$ is the inside heat transfer coefficient,

$\mathrm{h}_{\mathrm{i}}$ is the outside heat transfer coefficient, and

$\mathrm{k}_{\mathrm{ss}}$ is the thermal conductivity of stainless steel.

$\mathrm{Q}=\mathrm{U}_{\mathrm{o}} \mathrm{A}_{\mathrm{o}}\left(\mathrm{T}_{\text {Vapor }}-\mathrm{T}_{\text {Fluid }}\right)$

where: $U_{\mathrm{o}}$ is the overall heat-transfer coefficient, $\mathrm{A}_{\mathrm{o}}$ is the outside surface area of the tubes, $\mathrm{T}_{\text {Vapor }}$ is the temperature of the vapor, and $\mathrm{T}_{\text {Fluid }}$ is the temperature of the fluid.

\subsubsection{Reactor Slurry Cooler Before The Centrifuge (HE-104)}

After the first run with coal and NMP, it was noticed that there needed to be a heat exchanger installed in between the reactor and the centrifuge. This heat exchanger was designed constructed and installed to reduce the temperature of the reactor's contents from $202^{\circ} \mathrm{C}$ to the maximum operational temperature of the centrifuge $\left(100^{\circ} \mathrm{C}\right)$ in less than 30 minutes. Figure 1.18 shows the operational temperature profile this heat exchanger.

Heat Exchanger HE-104 was mounted vertically to ensure that the solids flowed from the top to the bottom. The tap water flowed on the shell side and the NMP/coal slurry on the tube-side. The overall average overall heat-transfer coefficient $\left(\mathrm{U}_{\mathrm{o}}\right)$ can be calculated for heat exchanger HE-104 cooling the NMP/coal mixture from the reactor using tap water. From Figure 1.18 , the temperature is cooled from $392^{\circ} \mathrm{F}$ to $200^{\circ} \mathrm{F}\left(200^{\circ} \mathrm{C}\right.$ to $\left.93^{\circ} \mathrm{C}\right)$ in 23 minutes. Using Equation 1.6 to calculate the average specific heat of $2.1-\mathrm{kJ} / \mathrm{kg}{ }^{\circ} \mathrm{C}$, the true average rate of heat transfer $\left(Q_{\text {avg }}\right)$ is $17.9 \mathrm{~kW}$. Substituting this into Equation 1.10, the average overall heat-transfer coefficient $\left(\mathrm{U}_{\mathrm{o}}\right)$ is calculated to be $230 \mathrm{~W} / \mathrm{m}^{2}{ }^{\circ} \mathrm{C}$. 


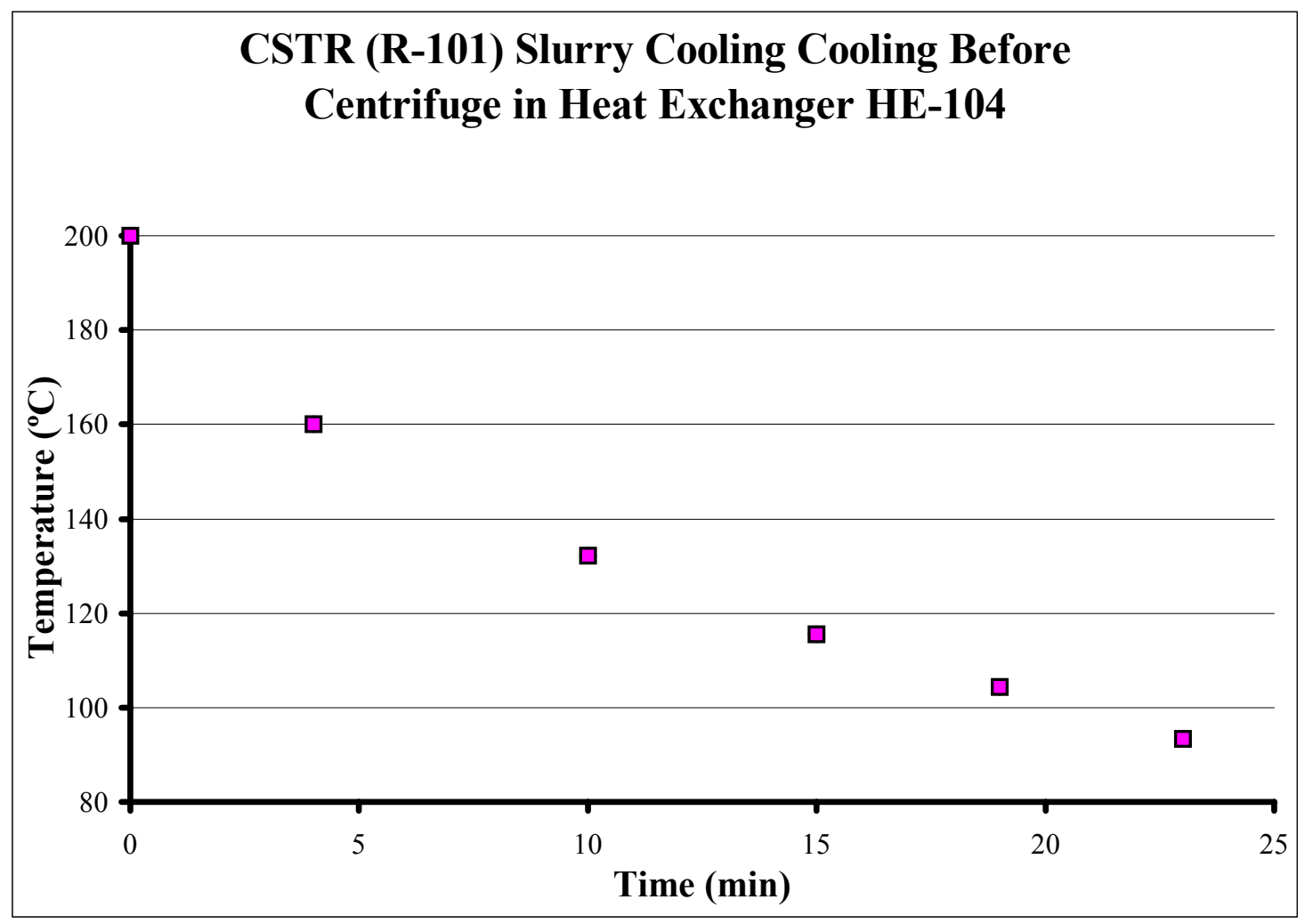

\section{Figure 1.18 CSTR (R-101) Slurry Cooling Before Centrifuge (Ct-101) With Supporting Heat Exchanger (HE-104)}

\subsubsection{Energy Balance on Wiped Thin Film Evaporator (TFE) (TFE-101)}

A Wiped Thin Film Evaporator (TFE) was used as the third stage of a continuous process for making coal-based carbon pitches. The solvent (NMP) in the CSTR (R-101) dissolves the coal. Then the dissolved portion of the coal is separated from the un-dissolved portion in the Centrifuge (Ct-101). The TFE is then used to drive off the majority $(72 \pm 2 \%)$ of the solvent from the dissolved portion of the coal. Then a Rotovap is used to drive off the most of the remaining solvent from the product before it is placed in a vacuum drying oven to complete the process.

Again, the TFE evaporation system thickens the filtrate (solvent and dissolved coal) by separating a majority of the solvent through an evaporation process from the filtrate.

Earlier, the five main operating parameters for the TFE that affect the quality of the product were detailed. Using the assumptions listed earlier, four of the five operating parameters are established. The temperature of the heating fluid $\left(T_{m}\right)$ from the supporting heat exchanger (HE-102) is the only unknown parameter. An energy balance over the TFE for pure water can approximate this temperature. 
The rate of heat transfer is needed to start the energy balance. Assuming that the inlet feed temperature $\left(T_{i n}\right)$ is less than that of the saturation temperature governed by the operating pressure of the vacuum pump, the required rate of heat transfer is $3.1 \mathrm{~kW}$. This is for a feed rate $\left(F_{\text {in }}\right)$ of 0.5 liters per minute while assuming $72 \%$ of NMP is evaporated off. Next, the temperature of the filtrate film is needed to perform an energy balance over the system. Figure 1.19 show the $\log$ of the saturation pressure $\left(\mathrm{P}^{\text {sat }}\right)$ of NMP vs. temperature $\left(\mathrm{T}^{\text {sat }}\right)$. The saturation temperature is the temperature at which the NMP is boiling for a set pressure. This temperature is the temperature of the filtrate film.

From Figure 1.19 the data are regressed to form the following equation for the saturation temperature $\left(\mathrm{T}^{\mathrm{sat}}\right)$.

$$
\mathrm{T}^{\mathrm{sat}}=\left\{\log _{10}\left(\mathrm{P}^{\mathrm{sat}}\right)+0.2349\right\} / 0.0156
$$

where: $\mathrm{T}^{\mathrm{sat}}\left[{ }^{\circ} \mathrm{C}\right]$ is the temperature of the filtrate film in the TFE, and $\mathrm{P}^{\mathrm{sat}}[\mathrm{mmHg}]$ is the operating pressure determined by the vacuum pump.

The maximum vacuum that can be drawn on the TFE by the vacuum pump (VP-101) is 5 $\mathrm{mmHg}$. With this vacuum, Equation 1.11 gives a filtrate film temperature $\left(\mathrm{T}_{\text {film }}\right)$ of $60^{\circ} \mathrm{C}$. Plugging the experimentally determined overall heat transfer coefficient for pure water and Marlotherm HT $\left(\mathrm{U}_{\mathrm{i} \text { water }}=150 \mathrm{~W} / \mathrm{m}^{2}{ }^{\circ} \mathrm{C}\right)$ into Equation 1.10 , the calculated set operating temperature for the heating fluid $\left(\mathrm{T}_{\mathrm{m}}\right)$ for the supporting heat exchanger (HE-102) is $245^{\circ} \mathrm{C}$.

The actual operating temperature was found to be $208^{\circ} \mathrm{C}$ leaving an $18 \%$ error. This error can be attributed to several sources: (1) the feed temperature for the NMP solution is at or above the film temperature in the TFE, while the feed temperature for the water case was atmospheric; (2) the feed rate for the NMP is about half that or plain water, (3) the higher operating temperature for the mineral oil could facilitate better heat transfer from the mineral oil, (4) the physical properties of water and NMP are very different, and/or (5) the supporting heat exchanger (HE-102) was larger than the one used in the water test, as a result it supplied the hot oil at a much higher rate. 


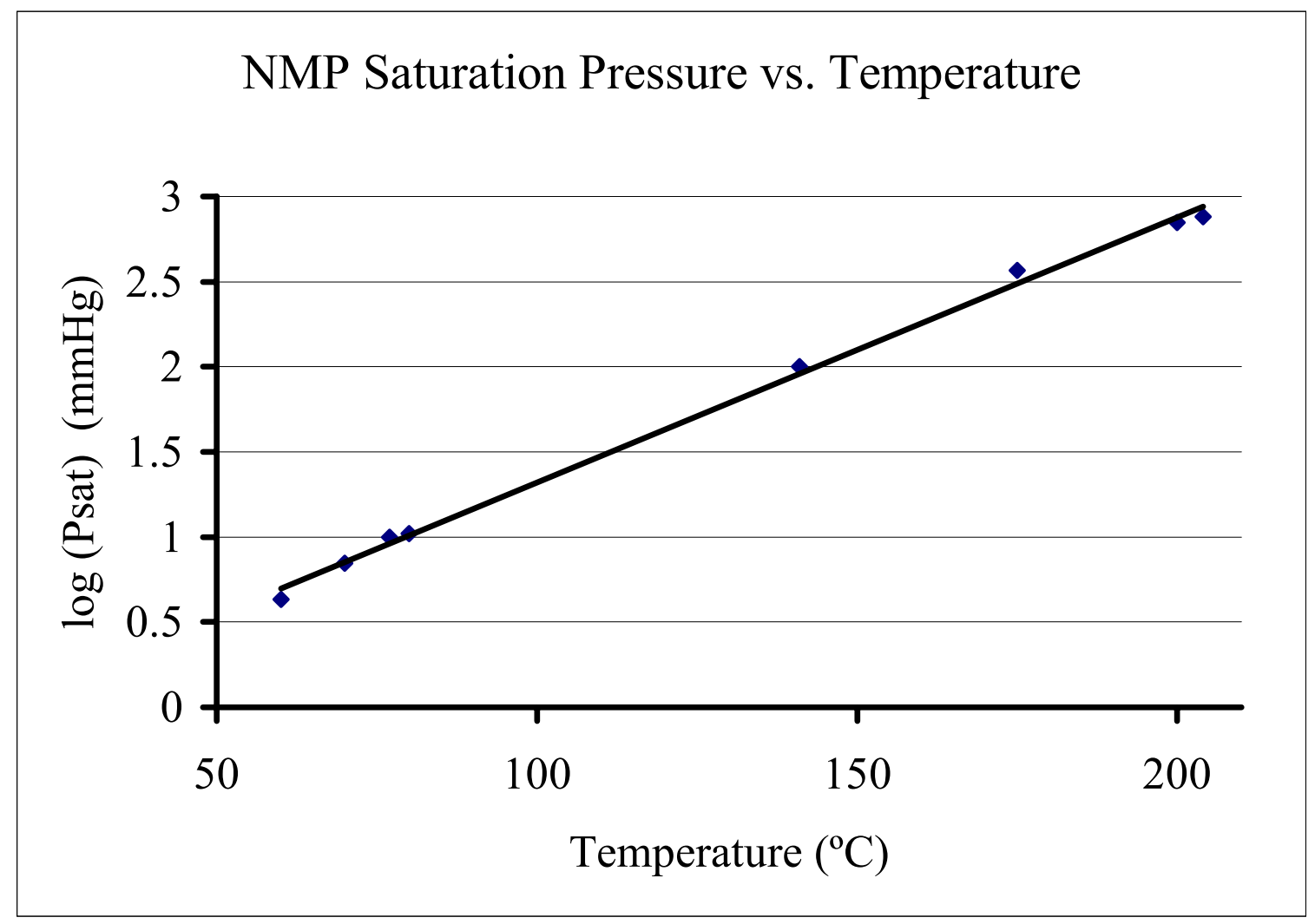

Figure 1.19 Log of NMP Saturation Pressure vs. Temperature

The five operating parameters for a normal run of the TFE are shown in Table 1.6. These operating parameters are from runs 12 and 13. These two runs were processed together through the TFE at one time. The TFE is a continuous unit, and is not easily shut down. The TFE takes about an hour to start up and about an hour to clean between runs and prepare for the next. Therefore running two or more runs through at once helps to reduce down time.

\subsubsection{Energy Balance on Rotary Evaporator (Rotovap)}

(RV-101)

The Rotovap operates much like a batch TFE. The Energy balance over this unit is fairly simple. The Rotovap takes the NMP concentration down from the 88 -weight $\%$ coming from the TFE to $50 \%$ by weight. The Rotovap processes 3 batches per run for a total of $3.5 \mathrm{~kg}$ extract and $25.5 \mathrm{~kg}$ of NMP. The total NMP driven off is $22 \mathrm{~kg}$ requiring a thermal load of approximately $12 \mathrm{MJ}$. The product, which is $50 \%$ product and $50 \%$ extract by weight, is then sent to the drying ovens.

The Rotovap has a condenser of $1.5 \mathrm{~m}^{2}$. This condenser is used to condense the majority of the vapors driven off. The Rotovap boils off NMP at a low temperature because of the low pressure pulled on the system by the vacuum pump (VP-101). 
Table 1.6 TFE Normal Operating Parameters

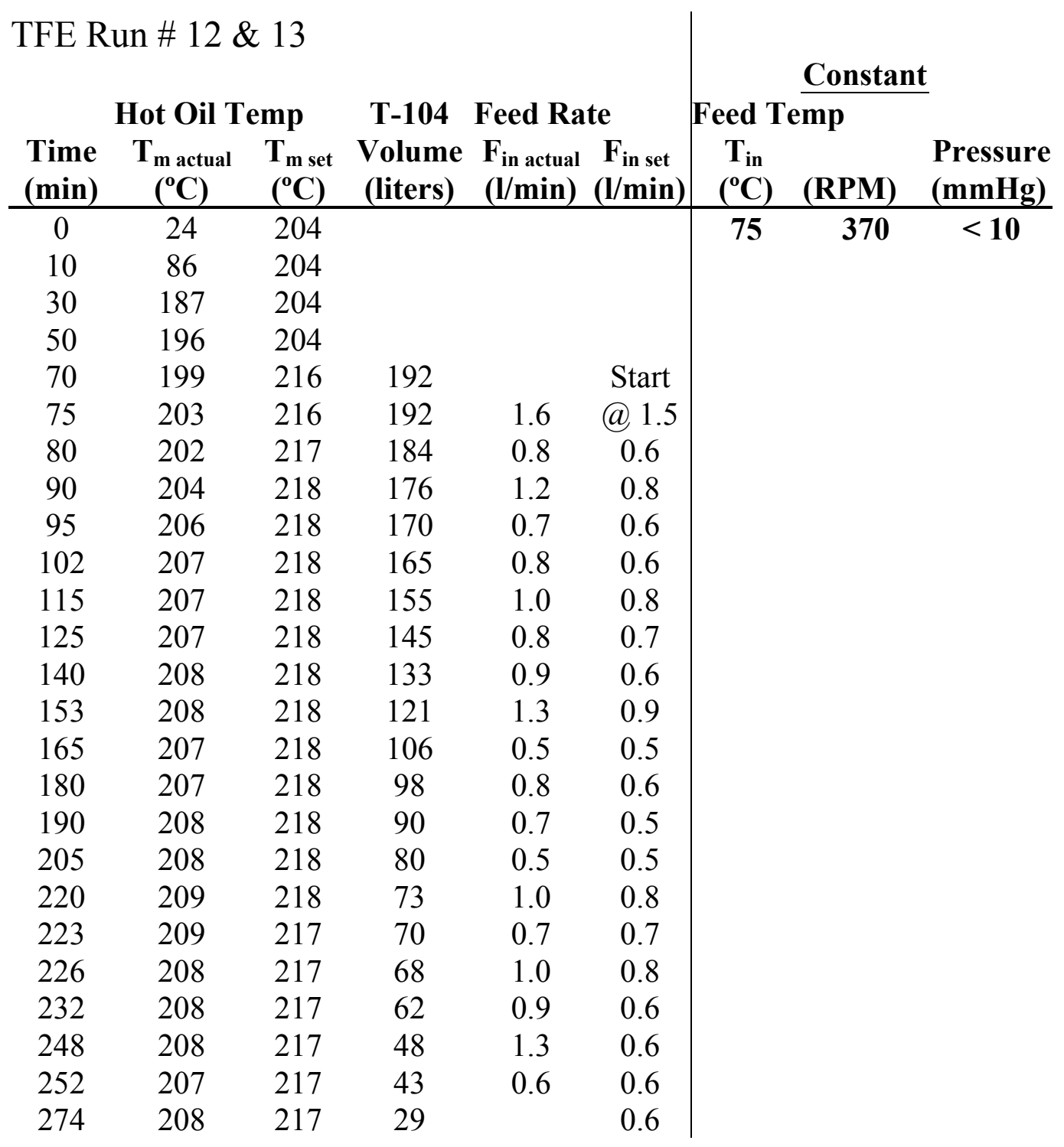

\subsubsection{Energy Balance on Drying Ovens (DO-101-3)}

Again, the drying ovens are used to vacuum dry the coal waste leaving the centrifuge, and the semisolid extract leaving the Rotovap. The drying ovens essentially bake off the $55 \%$ by weight NMP in the processed coal stream, and the remaining $50 \%$ NMP in the product stream from the Rotovap. The coal waste stream leaving the ovens resembles that of the fresh coal entering the reactor. It is essentially unchanged other than the ash content. The product stream from the ovens is the coal-based carbon pitch in the form a dry friable granular powder. 
The vacuum drying ovens use a nitrogen purge to carry the NMP vapors to the condenser (HE-105). Heat Exchanger HE-105 is the condenser for the drying ovens and vacuum trap for the vacuum pump (VP-102). This heat exchanger is made up of a 2-pass shell-and-tube heat exchanger mounted on top of a 20 -gallon NMP condensate collection tank (T-106). This tank sets on top of a 45 -gallon antifreeze sump (reservoir).

An air-operated diaphragm pump circulates the antifreeze from the 45-gallon sump through the tube side of the condenser (HE-105). This antifreeze is used as the cooling media to condense the NMP vapors that are driven off in the drying ovens. The NMP vapors leave the drying ovens and enter the freezer into the top left side of the collection tank. The vapor flows across the top of the collection tank and up into the condenser where it passes through a series of baffles. The vapor is condensed as it flows over the chilled tubes. The condensate flows counter currently down into the collection tank and is recycled to the reactor. The heat exchanger (HE-105), collection tank (T-105), and antifreeze sump are contained in an upright freezer.

\subsection{Conclusions}

The Coal Extraction Pilot Plant was designed and built to allow the preparation of significant quantities of coal extract and evaluate the mass and energy balance of the process. The Upper Powleton coal (WVGS 1342 in these experiments is about $36 \pm 2 \%$ soluble in NMP during a 60 -minute extraction period. As envisioned, $10 \mathrm{~kg}$ of coal is mixed with $100 \mathrm{~kg}$ of solvent in this experimental setup. Therefore, each run through the unit produces about 3.7 $\mathrm{kg}(8 \mathrm{lbs})$ of coal-based carbon extract.

Material and Energy balances have been calculated over the system. The question of solvent loss, or solvent retention, has been addressed. Using the result of the Ultimate Analysis, there is a solvent retention of about $0.7 \%$ by weight of processed coal in the extract and coal. Assuming the worst case, all the lost solvent is in the final product, the extract contains less than $2 \%$ NMP by weight.

The Coal Extraction Pilot Unit is now being utilized to mass produce coal-based carbon extract and evaluate different operating processes and economics.

In conclusion:

1. The Coal Extraction Pilot Unit was built and operated efficiently. This process scheme was developed for the extraction and recovery of large amounts of coal extract from coal.

2. The final product (coal based carbon pitch, reconstituted coal, or extract) has a very low ash content $(<0.6 \%$ calculated from the ultimate analysis).

3. Solvent recovery has been addressed, and was found that greater than $99.9 \%$ of the initial solvent was recovered. 
4. By using the Ultimate Analysis, an overall material balance can be done over the system. This material balance shows a yield of $36 \pm 2 \%$, a yield (MAF) of $39 \pm 2 \%$.

5. The CEPU has a MBC of less than $1 \%$, and a solvent retention of $0.69 \%$ by weight of raw coal processed.

6. The TFE is not the best piece of equipment to be utilized in this process. The TFE would work better where there is a liquid/liquid separation.

7. Water reduces NMP's ability to dissolve coal. As little as 3 mole $\%(0.6$ weight $\%$ ) will reduce the extraction yield to less than half.

8. The final reconstituted coal has an increase energy value of over 14,500 BTU/lb, as compared to the original Upper Powleton coal (WVGS13421), which has an energy value of $12,100 \mathrm{BTU} / \mathrm{lb}$.

9. The final reconstituted coal contains $30 \%$ volatile matter, as compared to the original Upper Powleton coal, which has $26 \%$ volatile matter.

10. The final reconstituted coal has $68 \%$ fixed carbon, as compared to the original Upper Powleton coal (WVGS13421), which has fixed carbon value of $65 \%$.

\subsection{Alterations to the Pilot Plant}

The most obvious question is: Why are all these steps required to remove the NMP from the coal extract? First, the TFE was designed to separate $70-75 \%$ volatile fluids from nonvolatile fluids. In this research, a volatile fluid was separated from a solid. In order for the TFE to operate continuously, enough NMP must be left in the "thickened coal extract" so it can flow. The effort is to minimize the amount of NMP in the coal extract and still have it flow. The rotary evaporator is designed to dry small quantities of material, which adhere to the walls of the container. When the coal extract dries, it no longer adheres to the container walls and a gum-like, thickened coal extract is produced. The material forms a large ball, limiting the effectiveness of drying with the Rotovap. The vacuum oven drying process is fairly slow - but very efficient.

It is possible that a single unit may be able to replace all three of the above drying units. Figure 1.20 and 1.21 show a double planetary mixer/vacuum dryer manufactured by Charles Ross and Son Company. This unit operates in a manner similar to a large Rotovap. However, instead of the vessel rotating in a hot oil bath, a set of blades (shown in Figures 1.22 and 1.23) in the vessel rotate and shear the thickened pitch. The extract continues to dry until it starts to break apart forming a granular like that solid (shown in Figure 1.23). This solid is then wiped around until essentially all the NMP is driven off. 
The walls of the planetary mixer are jacketed and heated by hot oil. The vessel is attached to a vacuum pump and condenser. The three drying steps in producing coal extract can be reduced into one. A single Ross double planetary mixer can perform the TFE, Rotovap, and drying oven steps. This use of this device greatly reduces process time and labor. Most significantly, it would minimize the need for physically handling the coal-based material.

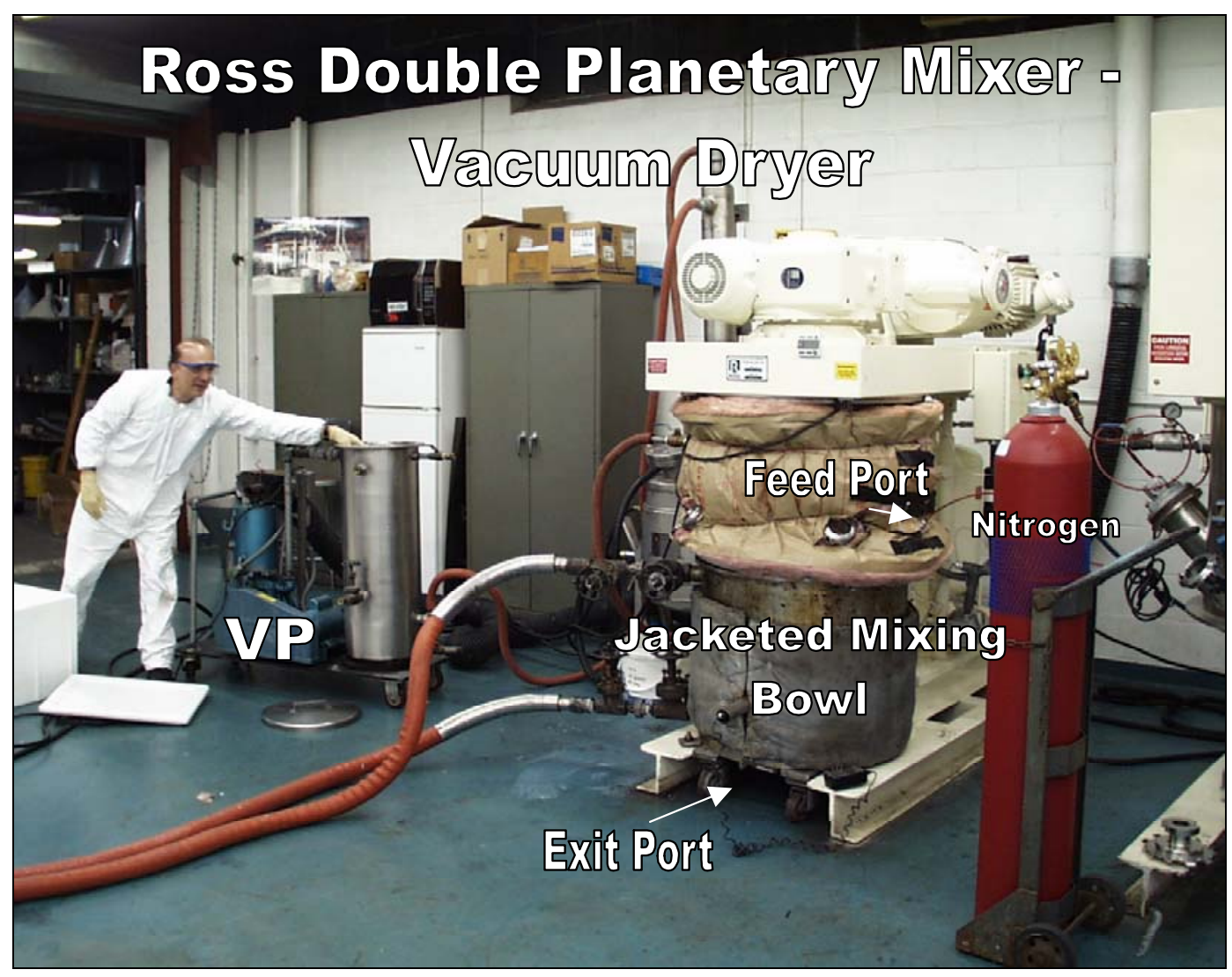

Figure 1.20 Charles Ross \& Son Co. (HDM-40) 40-gal Hydraulic Double Planetary Mixer 


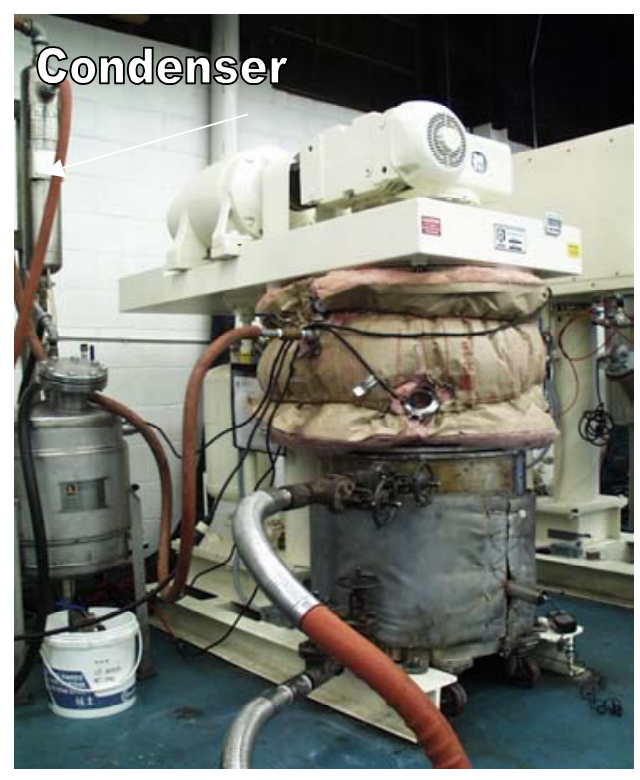

Figure 1. 21 Hydraulic Double Planetary Mixer with Supporting Condenser

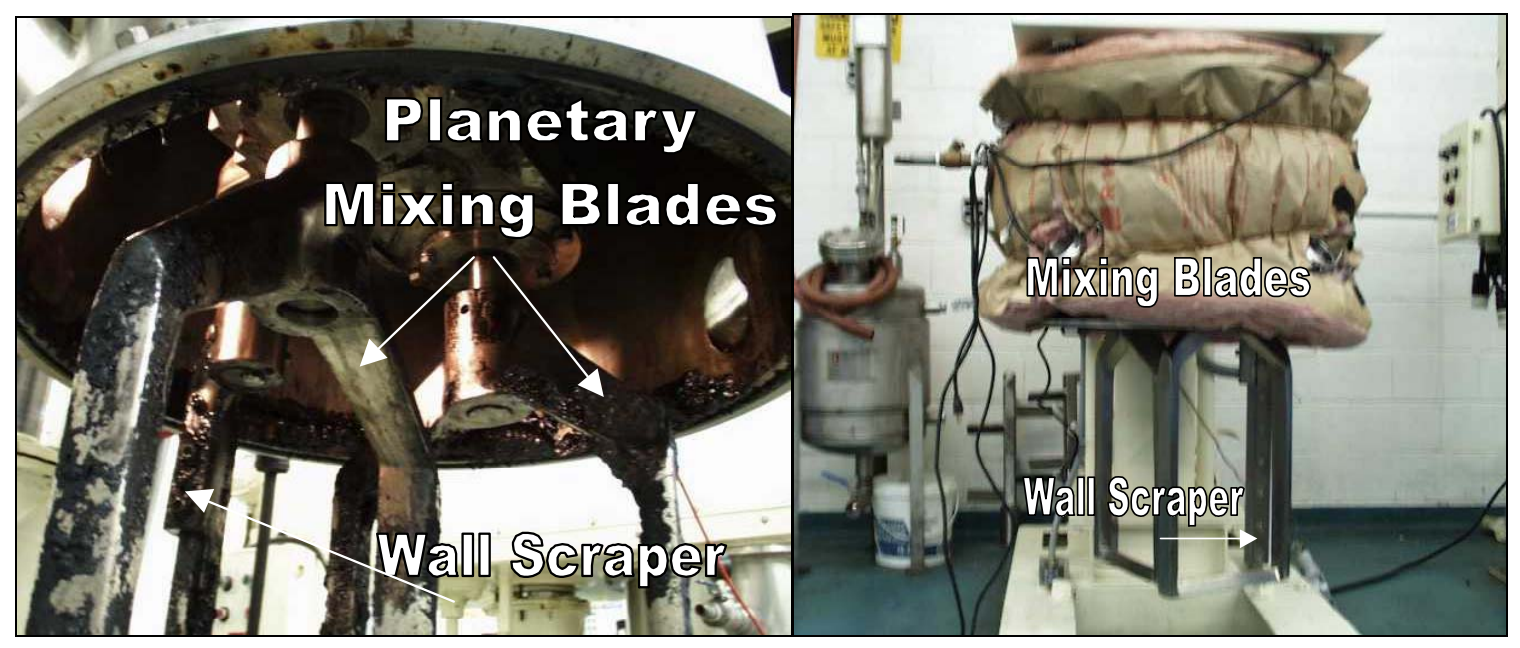

Figure 1. 22 Double Planetary Mixing Blades with Wall Scraper 


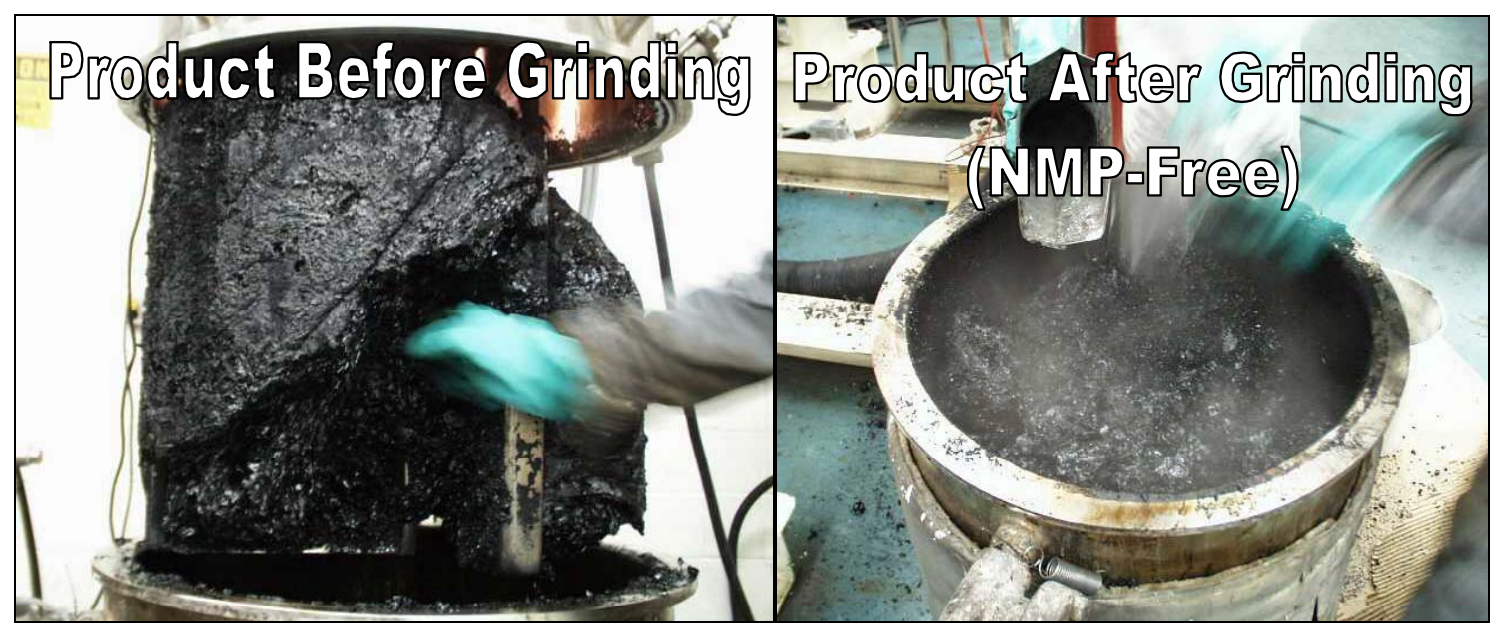

Figure 1.23 Double Planetary Mixing Blades With Product Before and After Grinding

A forty-gallon Ross blender was purchased and installed into the pilot plant. The unit was used to make over $200 \mathrm{lbs}$. of extract. The unit is clearly visible in Figure 1.24. A complete mass balance and energy balance for the system has not yet been completed. 


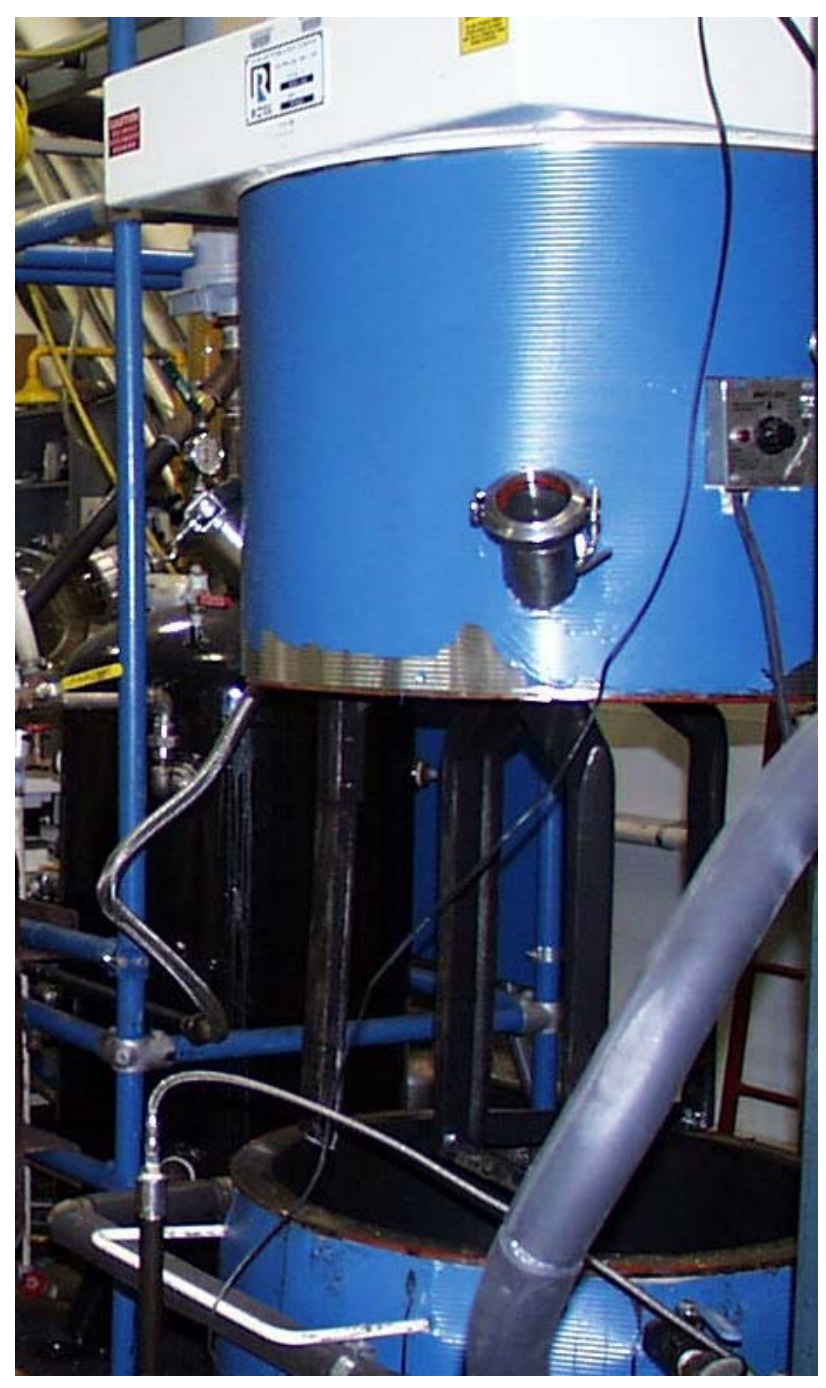

Figure 1.24 Ross HDM 40 Installed on CEPU 


\subsection{References}

1. American Society for Testing and Materials, Committee D-5 on Coal and Coke, ASTM Standards on Coal and Coke, American Chemical Society for testing and Materials, Philadelphia, PA 1984.

2. Asbury, R. S., Industrial Engineering Chemistry, Vol. 26, pg.1301, 1934.

3. Bland, Brian, Design Construction and Evaluation of Coal Extraction Pilot Plant to Manufacture Coal Based Carbon Pitch. Thesis, Department of Chemical Engineering, West Virginia University, Morgantown, WV 2000.

4. Bonifera, V.P. Jr., Economic Analysis of Integrated Process or The Production of Coal Derived Carbon Products. Thesis, Department of Chemical Engineering, West Virginia University, Morgantown, WV 1997.

5. Dryden, I. G. C., Fuel, Vol. 29, pg197, 1950.

6. Dryden, I. G. C., Fuel, Vol. 30, pg39, 1951.

7. Dryden, I. G. C., Fuel, Vol. 34, pg383, 1954.

8. Gadam, S.D., Coal Upgrading Under Mild Conditions Using Supersolvents, Graduate Thesis, West Virginia University, Department of Chemical Engineering, Morgantown, WV 1990.

9. GAF Corporation, Chemical Division, M-Pyrol-N-Methyl-2-Pyrrolidone, New York, NY 1972.

10. Gerstner, J.A., Laboratory investigation, Design and Economic Assessment of a low Temperature Coal De-ashing and Desulfurization process, Thesis, Department of Chemical Engineering, West Virginia University, Morgantown 1989.

11. Illingworth, S. R., Fuel, Vol. 34, pg.363, 1922.

12. Kirov, N.Y., BCURA Monthly Bulletin, 1960.

13. Leong, K., Coal Liquefaction at Mild Conditions Utilizing NMP and TMU Solvents, Undergraduate Thesis, West Virginia University, Department of Chemical Engineering, Morgantown, WV 1985.

14. Lowry, H. H., The Chemistry of Coal Utilization, Volume 1, John Whiley \& Sons, New York 1945.

15. Lowry, H. H., The Chemistry of Coal Utilization, Supplementary Volume 2, John 
Whiley \& Sons, New York 1962.

16. Marzec, A., Juwa, M., Betlej, K. an Sbokowwik, M., Fuel Processing Technology, Vol. 2, pp35-44, 1979.

17. Phillips, G.R., Solvent Extraction of West Virginia Coals in N-Methyl Pyrrolidone, Thesis, Department of Chemical Engineering, West Virginia University, Morgantown, WV 1988.

18. Renganathan, K., Beneficiation of Bituminous Coals Using Solvent Treatment At Mild Conditions, Thesis, Department of Chemical Engineering, West Virginia University, Morgantown, WV 1986.

19. Roy, J., Banerjee, P., and Singh, P. N., Indian Journal of Technology, 14, 1978.

20. Stewart, W.R., Design and Evaluation of a Batch Reactor System for Catalytic Coal Liquefaction, Thesis, Department of Chemical Engineering, West Virginia University, Morgantown, WV 1992.

21. Stiller, A. H., Sears, J. T., Hammack, R. W., United States Patent 4,272,356, June 9, 1981.

22. Stiller, A. H., Zondlo, J. W., Mintz, E. A. Jagodzinski, P. A. and Leong, C. W., "Interactions of solvents on Coal", Proceedings of the $2{ }^{\text {nd }}$ Annual Pittsburgh Coal Conference, p413, Sept. 1985.

23. Stoppes, M. C., Wheeler, R. V., Fuel, 3, pg63, 1924.

24. Volborth, A., Coal Science and Chemistry, Elsevier Science Publishers B.V., New York, NY, 1987.

25. Wise, W. S., Solvent Treatment of Coal, Crane Russak \& Co., New York, NY 1971.

26. Zondlo, J.W., Class notes ChE 224, Coal Processing, West Virginia University, Department of Chemical Engineering, Morgantown, WV Spring 1995.

27. Zondlo, J.W., Presentation to Dupont and New Car Carbon at West Virginia University about Coal Processing, West Virginia University, Department of Chemical Engineering, Morgantown, WV, July 2000. 


\section{Task 2.0 Investigations of Hydrogenation Conditions}

\subsection{Introduction}

Five different coal-derived liquids have been collected for testing as liquefaction solvents in order to produce precursors for carbon products. The first of these liquids is a processderived coal liquid from Antaeus Technical Services, Inc. in Alloy, West Virginia. This asreceived coal liquid is a mid-distillate by-product from the pyrolysis of an Eastern cakingtype bituminous coal to produce high quality char. The second and third liquids are distillate fractions of the original Antaeus coal liquid. The fourth and fifth coal liquids are heavy creosote oil and carbon black base \#1, obtained from Koppers Industries, Inc. in Pittsburgh, Pennsylvania. Both of these liquids are distillate fractions of coal tar derived from the production of metallurgical coke.

The products of the liquefaction reactions were separated into three different materials: unconverted coal, carbon pitch, and a possible recycle solvent. The resultant carbon pitch was tested as a precursor of carbon products. This was done using standard test procedures such as softening point, ash determination, coking value, and optical texture. Using these parameters, the products from the direct liquefaction of coal can be compared to cokes and pitches of commercial value today.

All of the materials and equipment used in the experiments are discussed along with the process of performing the hydrogenation reactions, extracting the products, and testing the final products.

Various chemicals and gases were used during the course of this investigation. The supplier and purity are listed below for each of the chemicals and gases. Tetrahydrofuran (THF) was used as a solvent during the product separation steps of the experiments. THF was obtained from Fisher Scientific and was the histological grade with purity listed as $99 \%$ or greater. It was used during all experiments as delivered. Argon and hydrogen were used as the gaseous atmosphere for the hydrogenation experiments. Nitrogen was used as an inert purge gas during vacuum drying. They were obtained as standard laboratory grade from AirGas of West Virginia. A medium-volatile bituminous coal (WVGS 13421) was obtained from the West Virginia Geological Survey. A proximate analysis was performed to determine its amount of moisture, fixed carbon, volatile matter, and ash. The results of this analysis along with some other characteristics of WVGS 13421 are shown in Table 2.1. An ultimate analysis was performed to determine the elemental composition of the coal and this can be seen in Table 2.2. The coal was ground to -20 mesh and dried overnight in a vacuum oven. The vacuum oven was set at $110^{\circ} \mathrm{C}$ with a nitrogen purge $(100 \mathrm{cc} / \mathrm{min})$ and a vacuum of 25 30 in $\mathrm{Hg}$.

Three coal-derived solvents were obtained for testing as hydrogenation solvents. The first is a mid-distillate coal liquid obtained from Antaeus Technical Services, Inc. This coal liquid was produced as a by-product from the production of a high quality char and is referred to as the Raw Antaeus Coal Liquid (RACL). This coal liquid was a very viscous 
fluid at ambient temperatures. The second is a heavy creosote oil ( $\mathrm{HCO})$, and the third is a carbon black base (CBB). The latter two samples were obtained from Koppers Industries, Inc and are considered to be similar but were obtained from Koppers at different times. However, the elemental composition was slightly different for the two Koppers' samples, and so they were treated as separate solvents. The elemental composition of each raw material is

\section{Table 2.1 Characteristics of WVGS 13421}

\begin{tabular}{|l|c|}
\hline \hline Coal Bed & Powellton \\
\hline County & Raleigh \\
\hline ASTM rank & mvb \\
\hline Mean-maximum reflectance of vitrinite & 1.111 \\
\hline Proximate analysis (as received) & 0.98 \\
\hline Moisture & 67.87 \\
\hline Fixed carbon & 27.96 \\
\hline Volatile matter & 3.19 \\
\hline Ash & \\
\hline Petrographic composition (\% volume) & 63.3 \\
\hline Vitrinite & 5.7 \\
\hline Exinite & 30.0 \\
\hline Inertinite &
\end{tabular}

Table 2.2 Elemental Compositions ${ }^{a}$ of WVGS 13421 and Coal-Derived Solvents

\begin{tabular}{||c|c|c|c|c|c|c||}
\hline \hline & $\begin{array}{c}\text { WVGS } \\
13421\end{array}$ & RACL & DACL-L & DACL-H & HCO & CBB \\
\hline $\mathrm{C}$ & $81.59 \%$ & $86.67 \%$ & $80.51 \%$ & $88.51 \%$ & $93.41 \%$ & $91.85 \%$ \\
\hline $\mathrm{H}$ & $4.56 \%$ & $6.76 \%$ & $7.74 \%$ & $5.72 \%$ & $5.14 \%$ & $5.51 \%$ \\
\hline $\mathrm{N}$ & $1.10 \%$ & $1.22 \%$ & $0.94 \%$ & $1.25 \%$ & $0.88 \%$ & $0.81 \%$ \\
\hline $\mathrm{S}$ & $0.73 \%$ & $0.72 \%$ & $0.61 \%$ & $0.76 \%$ & $0.55 \%$ & $0.67 \%$ \\
\hline $\mathrm{O}^{\mathrm{h}}$ & $4.82 \%$ & $4.54 \%$ & $10.19 \%$ & $3.62 \%$ & $0.02 \%$ & $1.11 \%$ \\
\hline $\begin{array}{c}\text { H/C Atomic } \\
\text { Ratio }\end{array}$ & 0.67 & 0.94 & 1.15 & 0.78 & 0.66 & 0.72 \\
\hline $\begin{array}{l}{ }^{1} \text { Elemental compositions are not on an ash free basis } \\
{ }^{\circ} \text { Deternined by difference }\end{array}$ \\
\hline
\end{tabular}


shown in Table 2.2. HCO was a pasty material that needed to be heated in order to process easily. CBB was fluid at ambient temperatures but contained some solid like matter entrained in the fluid. Therefore, it was also heated before processing. The Antaeus coal liquids were further split into two fractions before use as donor solvents. The raw liquids were vacuum distilled to approximately $50 \%$ by weight. The temperature was kept below $270^{\circ} \mathrm{C}$ so that pyrolysis reactions do not occur significantly. The light distillate (DACL-L, b.p. $<270{ }^{\circ} \mathrm{C}, 25-30$ in. $\mathrm{Hg}$ ) and residue (DACL-H, b.p. $>270{ }^{\circ} \mathrm{C}, 25-30 \mathrm{in} . \mathrm{Hg}$ ) were collected and stored in the cold room until time for use. DACL-L was fluid at ambient temperature and used without further preparation. DACL-H was a pitch-like solid material that was ground to -8 mesh. This material exhibited a softening point of $120^{\circ} \mathrm{C}$ and a coke yield of $46 \%$ by weight. The heavy creosote oil and carbon black base were used without further treatment. The elemental compositions of these solvents are shown in Table 2.2.

\subsection{Experimental Procedure for Hydrogenation Reactions}

\subsubsection{Overview of Hydrogenation Reaction}

The effectiveness of five coal-derived liquids as hydrogenation solvents in the direct hydrogenation of a medium-volatile bituminous coal was investigated. Initially, the reaction conditions for each solvent-coal mixture were kept identical. The temperature was $400{ }^{\circ} \mathrm{C}$, and the initial pressure was $1000 \mathrm{psig}$ of hydrogen in the cold reactor. The solvent-to-coal ratio was 3 to 1 by weight, and the reaction time was one hour. These are standard conditions that have been used before in the literature for reactions of coal and model hydrogen donor solvents, such as tetralin. The products of the hydrogenation reactions were extracted using THF in order to calculate the overall conversion (as THF solubles) for the solvent-coal mixture. A list of conditions for all the reactions is shown in Table 2.3. For each set of reaction conditions given in Table 2.3 (identified by the run number $1-12$ ), four repeated trials were performed. These trials are identified by the letters A, B, C, and D. Thus each individual run is identified by a number followed by a letter. The number corresponds to the reaction conditions given in Table 2.3. The letter refers to a specific trial (A, B, C, or D) of the same reaction conditions. Finally, the effects of changing the gas-phase pressure and atmosphere (runs five through ten on Table 2.3), and solvent-to-coal ratio (runs four, eleven, and twelve) were investigated.

The extract (THF soluble fraction) was then vacuum distilled to recover a possible recycle solvent and a distillation residue. The heavy distillation residue (termed "pitch" hereafter) was tested as a possible precursor for carbon-products. The effect of the distillation on the pitch characteristics was investigated. Several distillations were performed to remove different amounts of the light fraction (termed "recycle solvent" hereafter). The light distillate is given the name "recycle solvent" because in many similar processes this fraction is recycled to the reactor for further hydrogenation reactions. However, testing the effectiveness of the isolated recycle solvents as subsequent hydrogenation solvents is outside the scope of this research and is not performed. The softening point of the resultant pitch from each distillation was determined. The data from these distillations and softening points allowed the correlation of the amount of recycle solvent removed and the softening point of 
the resultant pitch. This correlation was used to produce a pitch by vacuum distillation with a target softening point of $120^{\circ} \mathrm{C}$. A process flow diagram for the overall experimental procedure is shown in Figure 2.1.

Table 2.3 Hydrogenation Reaction Conditions

\begin{tabular}{|c|c|c|c|c|c|c||}
\hline $\begin{array}{c}\text { Run } \\
\#\end{array}$ & Solvent & $\begin{array}{c}\text { Temperature } \\
\left({ }^{\circ} \mathrm{C}\right)\end{array}$ & Atmosphere & $\begin{array}{c}\text { Pressure } \\
(\text { psig Cold })\end{array}$ & $\begin{array}{c}\text { Solvent/Coal } \\
\text { Ratio }\end{array}$ & $\begin{array}{c}\text { Time } \\
(\mathrm{hr})\end{array}$ \\
\hline 1 & DACL-L & 400 & $\mathrm{H}_{2}$ & 1000 & $3 / 1$ & 1 \\
\hline 2 & DACL-H & 400 & $\mathrm{H}_{2}$ & 1000 & $3 / 1$ & 1 \\
\hline 3 & RACL & 400 & $\mathrm{H}_{2}$ & 1000 & $3 / 1$ & 1 \\
\hline 4 & HCO & 400 & $\mathrm{H}_{2}$ & 1000 & $3 / 1$ & 1 \\
\hline 5 & CBB & 400 & $\mathrm{H}_{2}$ & 1000 & $3 / 1$ & 1 \\
\hline 6 & CBB & 400 & Argon & 1000 & $3 / 1$ & 1 \\
\hline 7 & CBB & 400 & $\mathrm{H}_{2}$ & 500 & $3 / 1$ & 1 \\
\hline 8 & CBB & 400 & Argon & 500 & $3 / 1$ & 1 \\
\hline 9 & CBB & 400 & $\mathrm{H}_{2}$ & 100 & $3 / 1$ & 1 \\
\hline 10 & CBB & 400 & Argon & 100 & $3 / 1$ & 1 \\
\hline 11 & HCO & 400 & $\mathrm{H}_{2}$ & 1000 & $2 / 1$ & 1 \\
\hline 12 & HCO & 400 & $\mathrm{H}_{2}$ & 1000 & $1 / 1$ & 1 \\
\hline \hline
\end{tabular}

\subsubsection{Fluidized Sand Bath Preparation}

A Techne SBL-2 fluidized sand bath was used to heat the reactors during hydrogenation runs. A TECHNE TL-8D temperature controller regulated the sand bath temperature. The sand bath was filled three-quarters full with a -100 mesh aluminum oxide powder (sand). The sand bath was preheated to $25^{\circ} \mathrm{C}$ above the desired reaction temperature. The extra temperature accounted for the rapid loss in temperature that occurred when the cold reactors were plunged into the sand bath. 


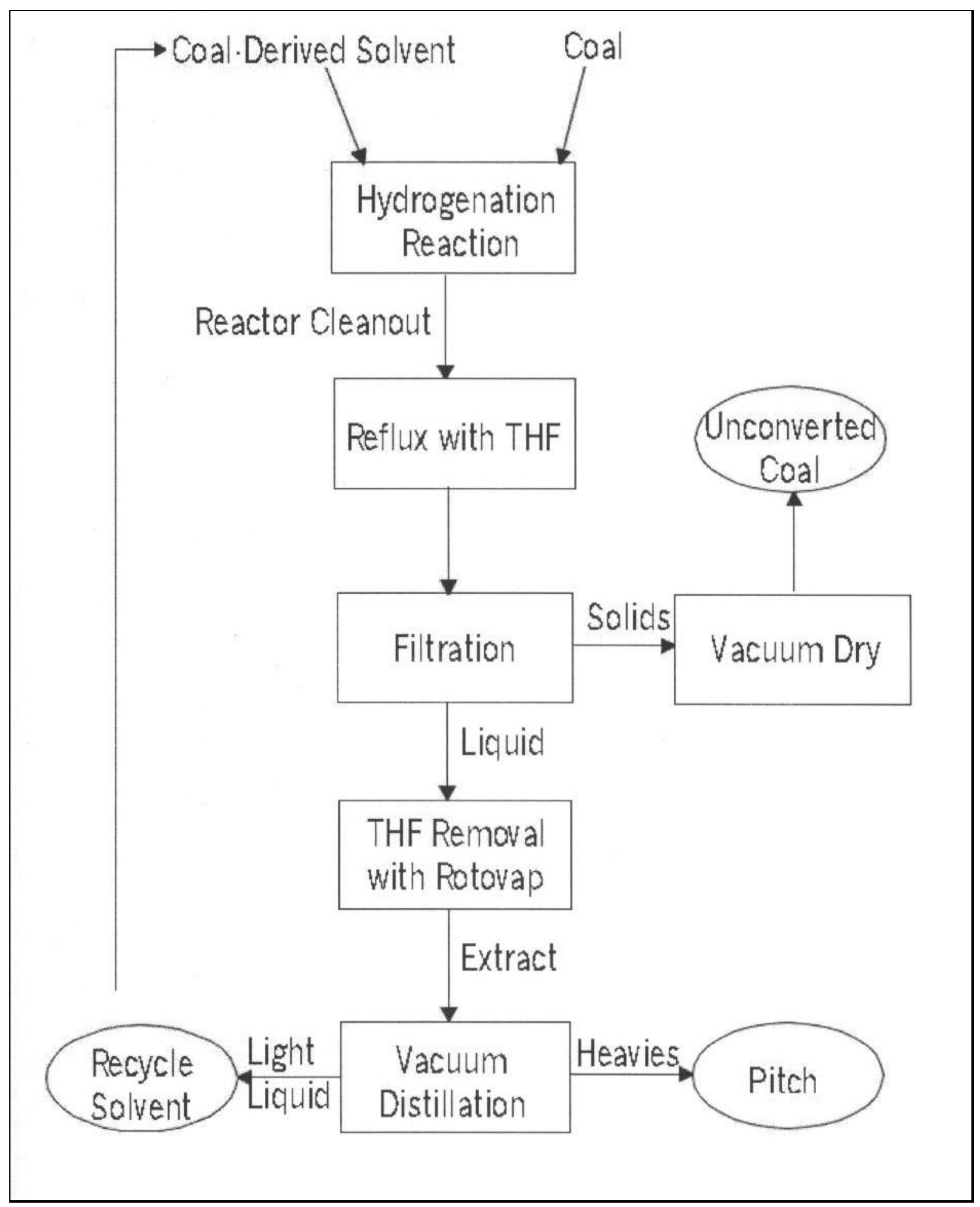

Figure 2.1 Experimental procedure flow sheet for production of carbon product precursors for coal hydrogenation reactions 


\subsubsection{Reactor Preparation}

Two tubing bomb microreactors (TBMR) were prepared for each hydrogenation run. The reactors were cleaned thoroughly before each use. The reactors had an internal volume of approximately $25 \mathrm{~cm}^{3}$ and were charged with about 5 grams of the coal/solvent mixture. A diagram of the TBMR is shown in Figure 2.2.

\subsubsection{Reactor Charging}

Reactants were weighed on an analytical balance (Denver Instruments Model A-200DS) to the nearest $0.1 \mathrm{mg}$ and then added to the reactor. The coal-derived solvent was placed in the reactor first. Since the coal liquids are viscous liquids, their mass was measured by difference. Once their mass was determined, the appropriate amount of coal was added to the reactor. The solvent-to-coal ratio was kept constant at 3 to 1. Finally, some ball bearings (usually five) were weighed and added to the reactor. These were introduced to help stir the contents of the reactor during reaction. Once all the reactants had been charged, the open end of the TBMR was sealed according to step 2.2.3.

\subsubsection{Gas Charging}

The reactions were run under either an argon or hydrogen atmosphere. This insured that oxygen would not react with the coal, promoting polymerization and the production of large insoluble coal fragments. Air was removed from the reactors by using a pressure purge cycle. The reactors were pressurized to 1000 psig initially with hydrogen or argon (depending on the specific hydrogenation run, see Table 2.3) and checked for leaks with a soap-water solution. The purge valve was then slowly opened to allow the reactor to reach atmospheric pressure again. This pressurization and release process was repeated two more times. Finally, the reactor was pressurized to the desired cold reaction pressure (see Table 2.3). The gas inlet valve of the tubular reactor was then closed, and the port on the gas inlet valve was sealed by screwing on a $1 / 4$-inch Swagelok plug.

\subsubsection{Reaction Procedure}

Once the reactors were charged with reactants and gas, they were placed in the reactor holder above the fluidized sand bath. The reactor holder was attached to a shaking mechanism used during the reaction. Once the reactors were secure in the holder, the shaking mechanism was turned on, and the speed was adjusted so that the reactors were vertically agitated at approximately $400 \mathrm{rpm}$ with a stroke of approximately 1.5 inches. The reactors were then heated by immersion into the sand bath by raising the sand bath using a winch-and-pulley system. The sand bath was raised until the hot sand completely covered the reactor bodies. The temperature of the bath was then adjusted to the desired reaction temperature. Finally, a stopwatch was started to count down from 58 minutes. The extra two minutes (total reaction time was to be $1 \mathrm{hr}$ ) allowed time to remove the reactors from the sand bath and quench the reaction. 


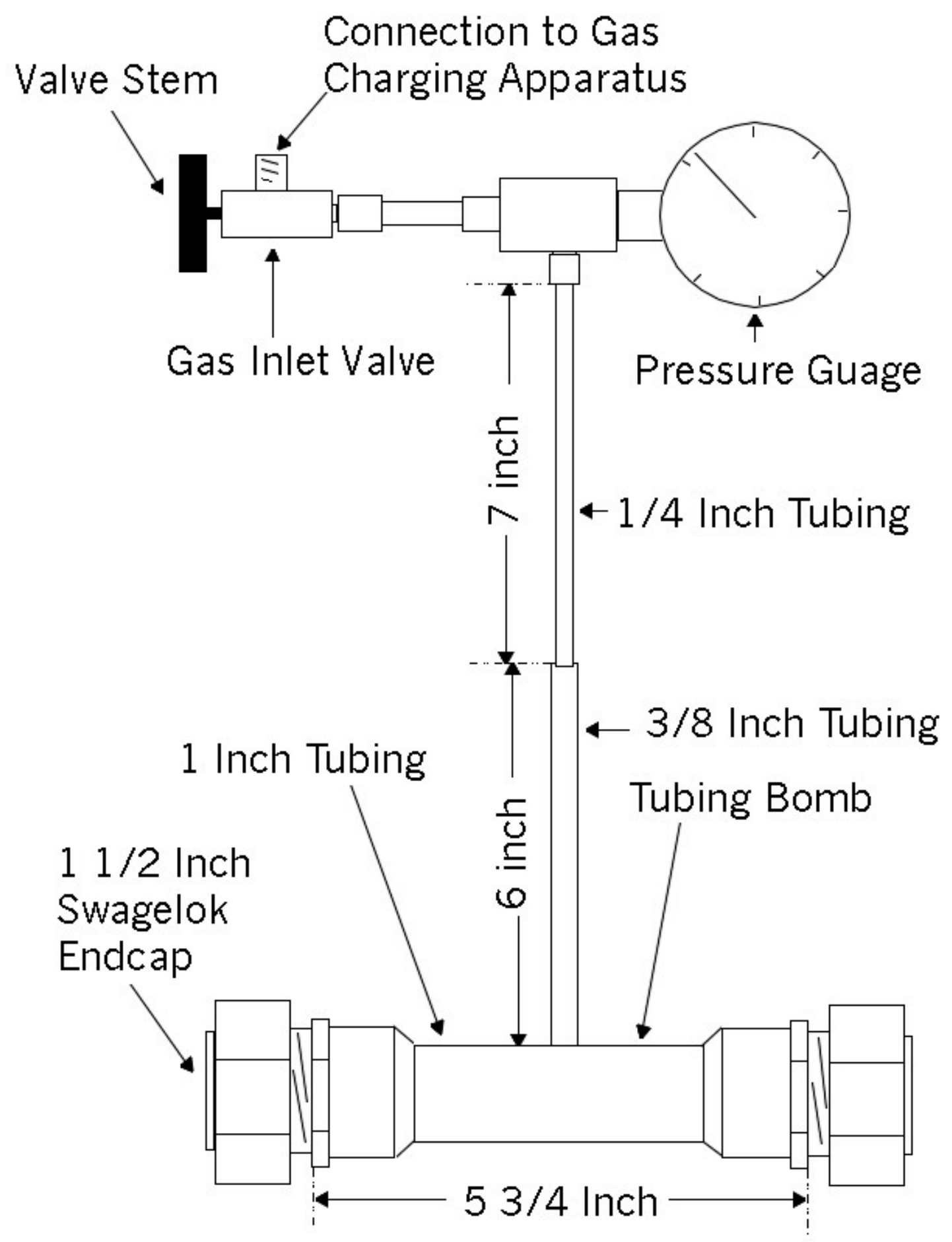

Figure 2.2 Diagram of tubing bomb microreactor (TBMR). 
Once the reaction time had elapsed (58 minutes), the sand bath was lowered by means of the winch-and-pulley system. The agitator was slowed down and then turned off. The reactors were removed from the holders and placed in a water bath. This served to cool the reactors quickly and quench the hydrogenation reactions.

\subsubsection{Product Collection}

The products of all the reactions except those using DACL-H or a solvent-to-coal ratio of $1 / 1$ were viscous fluids interlaced with some solid particles. The other two were more solid at ambient temperature and required a separate procedure for collecting the products of the reaction.

For all reactions except those with DACL-H or a solvent-to-coal ratio of $1 / 1$, the reactor was placed in a vise and the Swagelok plug was removed from the gas inlet valve. Then one end cap was slowly removed (so that the pressure could be released). The reactor was not vented through the gas inlet valve in order to keep the hydrogenation products in the main body (not the stem or pressure gauge) for ease of cleaning out the reactor. A small brush was used to clean any sand away from the reactor threads. The end cap was then replaced and only hand tightened. The reactor was flipped in the vice and the same procedure was used to loosen the other end cap.

A 500-mL flat-bottom boiling flask was placed in a clamp in a fume hood and fitted with a glass funnel. One end cap of the reactor was removed and the reactor was clamped over the funnel allowing its contents to drain into the funnel (when creosote oil, carbon black base, or raw Antaeus coal liquids were used as the hydrogenation solvent, a heat gun was used to help promote this process). Once the products stop dripping, the reactor was filled with THF. A stainless steel spatula was used to scrape the sides of the reactor while it contained THF. The THF was then drained into the boiling flask. This process was repeated several times until all the reaction mass was washed from the reactor. The transfer of the reaction mass from the reactor must be quantitative since the subsequent mass balance and processing steps depend on this procedure.

The end cap was then placed on the open end of the reactor, and the reactor was turned upside down over the funnel. The opposite end cap was now removed and the reactor was again washed with THF several times. Next, both end caps were removed and the final particulates left in the reactor were washed into the flask. The gas inlet valve was opened and THF was used to flush any matter from the reactor stem. The end caps were then scraped and washed out with THF. Finally, the funnel was washed with THF and removed from the flask.

For reactions using DACL-H or the lowest solvent-to-coal ratio, the reactor was chilled in dry ice for approximately 15 minutes before being opened. The end caps were removed by the procedure described above. The contents of the reactor were then chipped out using a metal spatula into a large metal pan. Once the contents of the reactor were removed to the pan, the products were then poured from the pan into a 500-mL flat bottom flask through a funnel. The pan, reactor, and funnel were finally washed with THF into the flask. 


\subsubsection{THF Extraction}

The 500-mL round bottom flask containing the coal/solvent/THF solution was filled (if needed) with fresh THF until it was approximately $3 / 4$ full. The flask was placed in a heating mantle and fitted with a simple water-cooled condenser. The cooling water was turned on and the heating mantle temperature was adjusted using a variac. The power was set so that a rolling boil $\left(\cong 75^{\circ} \mathrm{C}\right)$ was obtained. The contents of the flask were allowed to boil overnight (usually 12-16 hours).

The flask was then cooled to room temperature. The contents of the flask were centrifuged for 30 minutes at 2000 rotations-per-minute. The liquid portion of the centrifuged product was poured into a Buchner-type filtering funnel that was under vacuum suction. The filtering funnel was fitted with a pre-weighed piece of filter paper. Following filtration, the solid product (residue) was washed with THF while in the filter funnel. The residues produced by centrifugation and vacuum filtration were finally combined to form the THF insoluble fraction.

The filtrate was poured into a clean, weighed $250-\mathrm{mL}$ flat bottom flask. The THF in the filtrate was removed by rotary evaporation and collected for later use. The soluble product (extract) was set aside for further processing.

The THF insoluble residue was then placed in a vacuum oven $\left(80^{\circ} \mathrm{C}\right.$ and $\left.25-30 \mathrm{~mm} \mathrm{Hg}\right)$ and allowed to dry overnight (16-20 hours) under a nitrogen purge $(100 \mathrm{cc} / \mathrm{min})$. The dried residue was then weighed and the amount of THF insoluble product was calculated. This weight was used to calculate the overall conversion using Equation 2-1. The coal-alone conversion was also calculated using Equation 2-2 [13]. It should be noted that for purposes of calculating the coal-alone conversion, all the THF insoluble matter was assumed to come from the coal only and not from the added solvent.

$$
\begin{aligned}
& \% \text { Overall Conversion }=\frac{[\text { (Mass of Reactants })-(\text { Mass of THF Insolubles })]}{\text { Mass of Reactants }} \quad 2-1 \\
& \% \text { Coal Conversion }(\text { daf })=\frac{[\text { (Mass of Dry Coal })-(\text { Mass of THF Insolubles })]}{\text { Mass of Coal }(\text { daf })}
\end{aligned}
$$

\subsubsection{Product Isolation}

Once the THF was removed from the extract, the THF-soluble reaction products were separated using vacuum distillation. This was performed using the same procedure as described for separating the Antaeus coal tar into two fractions with one exception. The distillate was collected in a graduated cylinder in order to quantify the amount collected. The residue from the vacuum distillation was considered to be the soluble coal product (pitch). 
The distillate was the recycle solvent. The distillation was carried out several times (usually four) for each reaction. Each of these distillations collected a different amount of recycle solvent. The pitch and recycle solvent masses were calculated from each of these fractional distillations to determine the effect that the mass distilled had on the softening point of the pitch.

\subsection{Coal Extract Testing}

The properties of the coal extract pitches (i.e. high boiling distillate residues) collected from the hydrogenation reactions were evaluated as possible precursors for value-added carbon materials. These evaluation tests included softening point, coke yield, ash content, optical examination, and elemental analysis.

\subsubsection{Softening Point}

The softening point is required for the characterization of binder pitch, impregnation pitch, and pitch for fiber spinning. The softening point is a measure of the ability of the sample to flow and gives an indication of the rheological consistency of the sample.

The softening point was determined in duplicate using a Mettler FP80HT central processor and a Mettler FP83HT dropping cell following the ASTM D3104 procedure [4].

\subsubsection{Coking Value}

The coking value provides some insight into the amount of non-volatile matter in the sample. It also gives some indication of the sample's relative coke forming propensity. A desired value for coke yield is between 50 and 60 percent by weight. The coking value was determined using the West Virginia University (WVU) method. The sample (approximately $0.4-0.8$ grams) was placed in a pre-weighed porcelain crucible and immersed in coke breeze inside a larger crucible. The coke breeze allowed for the coking process to take place in the absence of oxygen. The sample was then heated to $600{ }^{\circ} \mathrm{C}$ at a rate of $5{ }^{\circ} \mathrm{C} / \mathrm{min}$ in a Fisher Scientific Isotemp Programmable Furnace Model 497. The sample was held at 600 ${ }^{\circ} \mathrm{C}$ for two hours and then cooled $10^{\circ} \mathrm{C} / \mathrm{min}$ until reaching room temperature. The crucible was then removed from the coke breeze and weighed. The coking value was determined using Equation 2-3.

Coking Value, $\%=\underline{(\text { Mass of Residue After Heating }) \times 100}$

(Mass of Original Sample)

This procedure was chosen for obtaining the coke yield so that the resultant coke sample's optical texture could be investigated. The long heating time allowed the characteristic domains of the sample to develop more fully. 


\subsubsection{Ash Content}

After the carbon content of a sample has been burned in air, the ash is the remaining residue. The ash is the inorganic oxides of the mineral matter that were present in the original coal. For most carbon products, the ash or inorganic portion of the sample is considered an impurity.

The ash content determination was carried out in a Fisher Scientific Isotemp Programmable Furnace Model 497. The ash content was determined according to the procedure specified by ASTM D2415 [3]. The sample was placed in a pre-weighed, partially covered crucible, set in the furnace, and heated at a rate of $8{ }^{\circ} \mathrm{C} / \mathrm{min}$ until the furnace reaches $500{ }^{\circ} \mathrm{C}$ in air. The heating rate was then changed to $4{ }^{\circ} \mathrm{C} / \mathrm{min}$ until the temperature reaches $750{ }^{\circ} \mathrm{C}$. The sample was held here for three hours in air and then cooled at a rate of $10^{\circ} \mathrm{C} / \mathrm{min}$ to ambient temperature. The final weight of the crucible and sample was determined, and the ash content was calculated from Equation 2-4.

$$
\text { Ash Content, } \%=\frac{(\text { Mass of Residual Ash }) \times 100}{\text { (Mass of Original Sample })}
$$

\subsubsection{Optical Texture}

Once the pitch sample has been converted to coke, the optical texture can be determined. The texture of a coke can be isotropic, anisotropic, or in between. The degree of optical texture can give some insight into the possible end uses for the carbon sample. It also indicates the surface and graphitization properties of each of the samples.

The optical texture of the sample was determined under a polarized-light optical microscope (Zeiss Axiostop, West Germany). The coke sample from the WVU method of coking was used to determine optical texture. The sample was embedded in epoxy resin and its cross section was polished on a Buchler Ltd. Metallurgical Apparatus with different textured abrasives. The sample was then observed under the Zeiss polarized light microscope and optical texture (isotropic or anisotropic) was determined. A camera was mounted on the polarized-light microscope to photograph the optical textures.

\subsubsection{Elemental Analysis}

An elemental analysis was performed on several representative pitches, residues, and recycle solvents. By combining this information with the elemental composition of the original hydrogenation reactants, an elemental balance could be performed for several different reaction conditions. A Flash EA 1112 series elemental analyzer from ThermoQuest was used for this analysis. Each sample was analyzed in triplicate, and any erroneous values 
were discarded before averaging the results.

\subsubsection{Error Calculation}

In order to determine the accuracy and consistency of the experimental procedures presented herein, a percent relative error was calculated for the different quantitative characterization techniques. Unless otherwise noted in this report, all error is reported as a relative error. The relative error is a percent deviation from the average value of the number reported. For example, an error of $\pm 2 \%$ in a value of 90.00 means that the range of error of 88.2 to 91.8 $\%$. The relative error was used to determine the error in two different values evaluated at the same conditions according to Equation 2-5.

$$
\% \text { Relative Error }=\frac{(\mid \text { Value } 1-\text { Value } 2 \mid) \times 100}{(\text { Value } 1+\text { Value } 2) / 2}
$$

\subsection{Results and Discussion}

The results of the production of carbon product precursors from the direct hydrogenation of coal are presented. Hydrogenation reactions were performed with various coal-derived solvents under similar conditions in order to determine the most effective solvent. Several of the reaction conditions (pressure, gas-phase composition, and solvent-to-coal ratio) were then varied so that their role in producing carbon-product precursors could be assessed. The results focus on the effect of gas pressure, gas-phase composition, solvent, and solvent-tocoal ratio on the conversion of coal to pitch-like materials and the properties of the pitch. The resultant materials were characterized by softening point, ash content, coking value, and optical texture. Products from several of the hydrogenation runs were also characterized by elemental analysis. Overall mass and ash balances were also performed for each run and are presented here. By evaluating the results, an optimum set of reaction conditions for the production of carbon-product precursors from coal can be established.

\subsubsection{Mass Balances}

A mass balance was performed for each hydrogenation reaction trial. The components of the mass balance were the amount of coal and solvent charged into the reactor and the amount of pitch, THF insolubles (residue), and recycle solvent collected after processing the hydrogenation products. The results of the mass balances can be seen in Table 2.4. The conditions for each hydrogenation run are given in Table 2.3.

For every reaction, a negative mass balance was observed, indicating a net loss of mass during the reaction and product separation steps. There are several different explanations for the negative mass balances. First, any product gas that may have formed during the reactions was not collected and quantified and thus is absent from the mass balance calculation. Typically the mass loss due to gas production could be between $2-5 \%$ by weight. Second, 
any light material that was produced during the hydrogenation reaction that condensed upon quenching of the reaction could have been lost during the THF extraction process. During THF removal by rotary evaporation, some of this lighter

material could have become volatile and collected with the removed THF causing a negative mass balance. These types of materials would also be lost during the initial period of vacuum distillation. Any very light material would not condense in the graduated cylinder with the other recycle solvent but would rather be collected in the cold trap of the vacuum pump. This was confirmed by periodically checking the cold trap of the vacuum pump. This was confirmed by periodically checking the cold trap of the vacuum pump for the presence of collected liquid (small amounts of liquid were usually present). Finally, the process of separating the hydrogenation products into different fractions (pitch, residue, and recycle solvent) required a significant amount of experimental transfers and procedures. Each of these procedures resulted in some amount of mass loss.

For the majority of the reaction runs listed in Table 2.4, the amount of mass loss was around $10 \%$ by weight or less. This is a reasonable result considering the procedures that were used in obtaining the product fractions. Several of the runs did, however, have a mass loss of greater than $10 \%$. This is most likely due to mass loss that occurred during the vacuum distillation step of product separation. The majority of the distillate (recycle solvent) needed to be heated in the condensing tube in order to make it flow into the graduated cylinder for collection. During these runs of greater lost mass, some recycle solvent could have been left in the condensing tube and therefore, not accounted for in the mass balance calculation. The presence of a negative mass balance will have some effects on further calculations and results below. The error from the mass balance is carried over into any calculation that depends on the data from the mass balance such as an ash balance or elemental balance. However, the accuracy of the mass balance (within 10\%) is good for this type of reaction system.

\subsubsection{Ash Balance}

An ash balance was performed on the products for all hydrogenation reactions and is presented in Table 2.5 with the various run conditions presented in Table 2.3. The ash content for each sample was determined according to the procedure outlined in Section 2.3.3 and on average had a relative error of less than $\pm 2 \%$. The ash content was determined for the reactants (coal and solvent) and for the pitch and THF insoluble portion of the products. Several samples of the recovered recycle solvent (the distillate) were tested for ash, but as 
expected the values were negligible. Therefore, the recycle solvent portion of the products was eliminated from the ash balance calculations. The weight of ash in each fraction was determined by multiplying the amount of each fraction (from the mass balance) by the ash percentage of that particular sample.

The results of the ash balance in Table 2.5 show a relatively random distribution of gain and loss of ash during the pitch production process. Since the ash balance depends on the mass of each fraction for calculation, any error in the mass balance will effect the ash balance results. Two figures, the ash present in the coal and the ash present in the THF insoluble residue, dominate the ash balance. The positive ash balance corresponds to the negative values of the mass balance calculations. If organic material is lost, the percentage of ash is elevated in the products, thus producing a positive ash balance. A negative ash balance could be attributed to incomplete separation of THF solubles and

insolubles. A small amount of THF solubles mixed with insolubles would cause a negative deviation in the ash content of the THF insolubles. Incomplete separation of the THF fractions could be attributed to the swelling of the filter paper and a large filter cake being formed during vacuum filtration. When the filtration slowed for these reasons, THF was removed by evaporation into the fume hood, depositing some THF solubles on the residue fraction, thus decreasing the ash content in the THF insoluble fraction and causing a negative ash balance.

\subsubsection{Solvent Evaluation}

The first goal of this research was to determine the effectiveness of five coal-derived liquids as hydrogen-donor solvents for coal. Each of the five coal liquids was used as a hydrogenation solvent under the same conditions. These reactions are run numbers one (1) through five (5) from Table 2.3. Initially, these liquids were characterized according to their ability to convert coal to THF soluble material. Two different methods of measuring their effectiveness were used: (1) the overall conversion based on the total feed (i.e. coal plus solvent) and (2) the coal-alone conversion based on the weight of coal only. The definition of these conversions is given by Equations 2.1 and 2.2, respectively in Section 2.2.8. The overall conversion for each of the solvents is given in Figure 2.3. These results show that the most effective solvent for conversion to THF solubles was DACL-L at $85.8 \pm 0.3 \%$. The least effective solvent was DACL-H at $71.5 \pm 1.3 \%$ overall conversion. As expected, the overall conversion achieved using RACL falls between the above two values at $76.0 \pm 1.5$ $\%$. The two liquids from Koppers Industries, Inc., HCO and CBB were also very effective according to Figure 2.3 with overall conversion values of $82.2 \pm 1.1 \%$ and $80.4 \pm 0.4 \%$, respectively.

The conversion results are quite different, however, when the coal-alone conversion is used. Figure 2.4 illustrates the effectiveness of the coal liquids as hydrogenation solvents on a coal-only basis. The order of conversion for each solvent when compared to the other solvents did not change by going to a coal-only basis. However, the absolute value of the conversion changed drastically for each solvent. The coal-alone conversion for reactions using DACL-L was $46.7 \pm 1.3 \%$ while a conversion of $-12.5 \pm 1.8 \%$ was calculated for 
DACL-H reactions. The negative conversion signified that not only was very little of the coal converted to THF solubles, but also some of the hydrogenation solvent (DACL-H) was insoluble in THF due to possible coking reactions. The coal-alone conversion for the hydrogenation reactions using RACL, $\mathrm{HCO}$, and $\mathrm{CBB}$ were $7.3 \pm 1.5 \%, 30.8 \pm 2.2 \%$, and $23.2 \pm 1.6 \%$, respectively.

The reason for the large difference between the values of the two methods of calculating the conversions is that the overall conversion includes the solvent in its calculation, while the coal-alone conversion does not. Because the coal liquids (solvents) exhibited a high solubility in THF to begin with and the use of high solvent-to-coal ratio ( $3 / 1$ by weight), the majority of the THF soluble

Table 2.4 Overall Mass Balances of the Coal Hydrogenation Reactions 


\begin{tabular}{|c|c|c|c|c|c|c|c|c|c|c|}
\hline Run & Trial & $\begin{array}{c}\text { In } \\
\text { Coal }(g)\end{array}$ & $\begin{array}{c}\text { In } \\
\text { Solvent }(\mathrm{g})\end{array}$ & $\begin{array}{l}\text { Total } \\
\text { In (g) }\end{array}$ & $\begin{array}{c}\text { Out } \\
\text { Pitch (g) }\end{array}$ & $\begin{array}{c}\text { Out } \\
\text { THF Ins. (g) }\end{array}$ & $\begin{array}{l}\text { Out } \\
\text { Rec. Solv. } \\
\text { (g) }\end{array}$ & $\begin{array}{l}\text { Total } \\
\text { Out (g) }\end{array}$ & $\begin{array}{l}\text { Out - In } \\
\text { (g) }\end{array}$ & $\mid \begin{array}{c}(\text { Out-In)/ln } \\
(\%)\end{array}$ \\
\hline 1 & A & 8.3812 & 25.1046 & 33.4858 & 8.5037 & 4.6171 & 16.6769 & 29.7977 & -3.6881 & -11.0 \\
\hline 1 & B & 8.4897 & 25.4679 & 33.9576 & 12.3280 & 4.7903 & 14.4829 & 31.6012 & -2.3564 & -6.9 \\
\hline 1 & C & 8.3809 & 25.1423 & 33.5232 & 9.6637 & 4.8612 & 15.3188 & 29.8437 & -3.6795 & -11.0 \\
\hline 1 & D & 8.4776 & 25.4351 & 33.9127 & 6.9489 & 4.8407 & 17.4629 & 29.2525 & -4.6602 & -13.7 \\
\hline 2 & A & 5.9996 & 17.9994 & 23.999 & 17.2400 & 6.6085 & 0.0000 & 23.8485 & -0.1505 & -0.6 \\
\hline 2 & B & 5.9988 & 17.9999 & 23.9987 & 16.2053 & 6.6643 & 0.0000 & 22.8696 & -1.1291 & -4.7 \\
\hline 2 & C & 6.0008 & 18.0008 & 24.0016 & 12.7595 & 6.8085 & 1.9900 & 21.558 & -2.4436 & -10.2 \\
\hline 2 & D & 6.0009 & 17.9990 & 23.9999 & 9.5452 & 7.2738 & 3.9600 & 20.779 & -3.2209 & -13.4 \\
\hline 3 & A & 7.6118 & 22.8324 & 30.4442 & 9.9431 & 6.9708 & 10.2900 & 27.2039 & -3.2403 & -10.6 \\
\hline 3 & B & 7.6541 & 22.9600 & 30.6141 & 11.5130 & 7.1990 & 9.7100 & 28.422 & -2.1921 & -7.2 \\
\hline 3 & C & 7.6744 & 23.0200 & 30.6944 & 8.8603 & 7.2135 & 11.2000 & 27.2738 & -3.4206 & -11.1 \\
\hline 3 & D & 8.2833 & 24.8500 & 33.1333 & 12.2342 & 8.6765 & 9.0800 & 29.9907 & -3.1426 & -9.5 \\
\hline 4 & A & 8.7873 & 26.3643 & 35.1516 & 11.0642 & 6.0297 & 14.6300 & 31.7239 & -3.4277 & -9.8 \\
\hline 4 & B & 8.8595 & 26.5785 & 35.438 & 5.4141 & 6.4669 & 21.2400 & 33.121 & -2.317 & -6.5 \\
\hline 4 & C & 8.8076 & 26.4215 & 35.2291 & 7.5975 & 6.2503 & 17.1900 & 31.0378 & -4.1913 & -11.9 \\
\hline 4 & D & 8.9488 & 26.8472 & 35.796 & 6.4161 & 6.5263 & 17.4800 & 30.4224 & -5.3736 & -15.0 \\
\hline 4 & $\mathrm{E}$ & 8.7055 & 26.1183 & 34.8238 & 7.1096 & 6.9566 & 20.2183 & 34.2845 & -0.5393 & -1.5 \\
\hline 5 & A & 9.0940 & 27.2847 & 36.3787 & 8.6773 & 6.9411 & 19.1700 & 34.7884 & -1.5903 & -4.4 \\
\hline 5 & B & 8.9570 & 26.8698 & 35.8268 & 7.1536 & 7.0941 & 20.6700 & 34.9177 & -0.9091 & -2.5 \\
\hline 5 & C & 8.8303 & 26.4937 & 35.324 & 5.6086 & 6.9512 & 21.2800 & 33.8398 & -1.4842 & -4.2 \\
\hline 5 & D & 8.8314 & 26.4938 & 35.3252 & 8.5937 & 7.0349 & 19.2400 & 34.8686 & -0.4566 & -1.3 \\
\hline 6 & A & 8.6048 & 25.8137 & 34.4185 & 6.0769 & 7.2494 & 16.9400 & 30.2663 & -4.1522 & -12.1 \\
\hline 6 & B & 9.0270 & 27.0806 & 36.1076 & 4.7830 & 8.1360 & 20.5800 & 33.499 & -2.6086 & -7.2 \\
\hline 6 & C & 8.5940 & 25.7836 & 34.3776 & 3.4138 & 7.9002 & 21.8700 & 33.184 & -1.1936 & -3.5 \\
\hline 6 & D & 8.9388 & 26.8162 & 35.755 & 6.5164 & 7.8078 & 19.1700 & 33.4942 & -2.2608 & -6.3 \\
\hline 7 & A & 8.5583 & 25.6744 & 34.2327 & 6.4744 & 7.0102 & 17.7300 & 31.2146 & -3.0181 & -8.8 \\
\hline 7 & B & 8.9103 & 26.7293 & 35.6396 & 5.3671 & 7.2512 & 21.4800 & 34.0983 & -1.5413 & -4.3 \\
\hline 7 & C & 8.6285 & 25.8895 & 34.518 & 5.4428 & 7.7470 & 18.8400 & 32.0298 & -2.4882 & -7.2 \\
\hline 7 & D & 8.8169 & 26.4519 & 35.2688 & 7.7059 & 7.7566 & 17.9700 & 33.4325 & -1.8363 & -5.2 \\
\hline
\end{tabular}

Table 2.4 (Continued) Overall Mass Balances of the Coal Hydrogenation Reactions 


\begin{tabular}{|c|c|c|c|c|c|c|c|c|c|c|}
\hline Run & Trial & $\begin{array}{c}\text { In } \\
\text { Coal }(g)\end{array}$ & $\begin{array}{c}\text { In } \\
\text { Solvent }(g)\end{array}$ & $\begin{array}{l}\text { Total } \\
\text { In (g) }\end{array}$ & $\begin{array}{c}\text { Out } \\
\text { Pitch }(g)\end{array}$ & $\begin{array}{c}\text { Out } \\
\text { THF Ins. (g) }\end{array}$ & $\begin{array}{c}\text { Out } \\
\text { Rec. Solv. } \\
\text { (g) }\end{array}$ & $\begin{array}{l}\text { Total } \\
\text { Out (g) }\end{array}$ & $\begin{array}{l}\text { Out - In } \\
\text { (g) }\end{array}$ & $\left(\begin{array}{c}\text { (Out-ln)/In } \\
(\%)\end{array}\right.$ \\
\hline 8 & A & 8.9144 & 26.7416 & 35.656 & 7.3802 & 8.0749 & 17.6100 & 33.0651 & -2.5909 & -7.3 \\
\hline 8 & B & 8.5729 & 25.7200 & 34.2929 & 3.9747 & 7.6985 & 20.1200 & 31.7932 & -2.4997 & -7.3 \\
\hline 8 & C & 8.8134 & 26.4439 & 35.2573 & 6.1858 & 8.3588 & 18.5200 & 33.0646 & -2.1927 & -6.2 \\
\hline 8 & D & 8.6033 & 25.8076 & 34.4109 & 4.9405 & 7.8483 & 19.6000 & 32.3888 & -2.0221 & -5.9 \\
\hline 9 & A & 8.9656 & 26.8936 & 35.8592 & 8.7617 & 8.2419 & 17.1200 & 34.1236 & -1.7356 & -4.8 \\
\hline 9 & B & 8.6900 & 26.0717 & 34.7617 & 5.4339 & 8.1171 & 19.1000 & 32.651 & -2.1107 & -6.1 \\
\hline 9 & C & 9.1626 & 27.4904 & 36.653 & 5.6319 & 8.4754 & 18.9100 & 33.0173 & -3.6357 & -9.9 \\
\hline 10 & A & 8.5836 & 25.7540 & 34.3376 & 7.3670 & 8.5019 & 15.8400 & 31.7089 & -2.6287 & -7.7 \\
\hline 10 & $B$ & 9.0234 & 27.0691 & 36.0925 & 4.7132 & 8.9974 & 18.9400 & 32.6506 & -3.4419 & -9.5 \\
\hline 10 & C & 8.6621 & 25.9863 & 34.6484 & 6.4714 & 7.9306 & 17.1300 & 31.532 & -3.1164 & -9.0 \\
\hline 10 & D & 8.8383 & 26.5110 & 35.3493 & 6.1156 & 7.9726 & 17.4600 & 31.5482 & -3.8011 & -10.8 \\
\hline 11 & A & 11.7463 & 23.4898 & 35.2361 & 7.2230 & 8.5762 & 14.3000 & 30.0992 & -5.1369 & -14.6 \\
\hline 11 & B & 11.5104 & 23.0186 & 34.529 & 8.0716 & 8.7815 & 15.2900 & 32.1431 & -2.3859 & -6.9 \\
\hline 11 & C & 11.6680 & 23.3358 & 35.0038 & 6.8942 & 9.1683 & 15.8000 & 31.8625 & -3.1413 & -9.0 \\
\hline 11 & D & 11.5366 & 23.0717 & 34.6083 & 6.9836 & 8.6664 & 17.2200 & 32.87 & -1.7383 & -5.0 \\
\hline 12 & A & 14.9673 & 14.9673 & 29.9346 & 8.1438 & 10.7303 & 8.5800 & 27.4541 & -2.4805 & -8.3 \\
\hline 12 & B & 15.1486 & 15.1480 & 30.2966 & 7.5261 & 11.1392 & 9.5600 & 28.2253 & -2.0713 & -6.8 \\
\hline 12 & C & 15.7095 & 15.7103 & 31.4198 & 6.2607 & 11.1933 & 9.7100 & 27.164 & -4.2558 & -13.5 \\
\hline$\sqrt{12}$ & D & 15.4615 & 15.4618 & 30.9233 & 8.1711 & 11.1289 & 9.2400 & 28.54 & -2.3833 & -7.7 \\
\hline
\end{tabular}


Table 2.5 Ash Balance of Hydrogenation Reactants and Products

\begin{tabular}{|c|c|c|c|c|c|c|c|c|c|}
\hline Run & Trial & $\begin{array}{l}\text { Ash In } \\
\text { Coal (g) }\end{array}$ & $\begin{array}{l}\text { Ash In } \\
\text { Solv. (g) }\end{array}$ & $\begin{array}{c}\text { Total Ash } \\
\ln (\mathrm{g})\end{array}$ & $\begin{array}{l}\text { Ash Out } \\
\text { Pitch (g) }\end{array}$ & $\begin{array}{c}\text { Ash Out THF } \\
\text { Ins. (g) }\end{array}$ & $\begin{array}{c}\text { Total Ash } \\
\text { Out (g) }\end{array}$ & $\begin{array}{l}\text { Out - In } \\
\text { (g) }\end{array}$ & $\begin{array}{c}(\text { Out-ln)/ln } \\
(\%)\end{array}$ \\
\hline 1 & A & 0.6030 & 0.0000 & 0.6030 & 0.0094 & 0.5333 & 0.5426 & -0.0604 & -10.0 \\
\hline 1 & B & 0.6108 & 0.0000 & 0.6108 & 0.0100 & 0.5207 & 0.5308 & -0.0801 & -13.1 \\
\hline 1 & C & 0.6030 & 0.0000 & 0.6030 & 0.0100 & 0.5882 & 0.5982 & -0.0048 & -0.8 \\
\hline 1 & D & 0.6100 & 0.0000 & 0.6100 & 0.0025 & 0.5228 & 0.5253 & -0.0846 & -13.9 \\
\hline 2 & A & 0.4317 & 0.0243 & 0.4560 & 0.0000 & 0.4011 & 0.4011 & -0.0548 & -12.0 \\
\hline 2 & B & 0.4316 & 0.0243 & 0.4559 & 0.0045 & 0.4538 & 0.4584 & 0.0025 & 0.5 \\
\hline 2 & C & 0.4318 & 0.0243 & 0.4561 & 0.0031 & 0.4837 & 0.4869 & 0.0308 & 6.8 \\
\hline 2 & D & 0.4318 & 0.0243 & 0.4561 & 0.0029 & 0.5110 & 0.5139 & 0.0578 & 12.7 \\
\hline 3 & A & 0.5477 & 0.0215 & 0.5691 & 0.0026 & 0.6012 & 0.6039 & 0.0347 & 6.1 \\
\hline 3 & $B$ & 0.5507 & 0.0216 & 0.5723 & 0.1036 & 0.5604 & 0.6641 & 0.0918 & 16.0 \\
\hline 3 & C & 0.5522 & 0.0216 & 0.5738 & 0.0020 & 0.5558 & 0.5578 & -0.0160 & -2.8 \\
\hline 3 & D & 0.5960 & 0.0234 & 0.6193 & 0.0032 & 0.5809 & 0.5841 & -0.0353 & -5.7 \\
\hline 4 & A & 0.6322 & 0.0000 & 0.6322 & 0.0023 & 0.7169 & 0.7193 & 0.0870 & 13.8 \\
\hline 4 & $B$ & 0.6374 & 0.0000 & 0.6374 & 0.0004 & 0.7146 & 0.7150 & 0.0776 & 12.2 \\
\hline 4 & C & 0.6337 & 0.0000 & 0.6337 & 0.0014 & 0.6644 & 0.6658 & 0.0321 & 5.1 \\
\hline 4 & D & 0.6439 & 0.0000 & 0.6439 & 0.0015 & 0.8452 & 0.8467 & 0.2028 & 31.5 \\
\hline 4 & $E$ & 0.6264 & 0.0000 & 0.6264 & 0.0036 & 0.8035 & 0.8071 & 0.1807 & 28.9 \\
\hline 5 & A & 0.6543 & 0.0132 & 0.6675 & 0.0039 & 0.7101 & 0.7140 & 0.0465 & 7.0 \\
\hline 5 & B & 0.6445 & 0.0130 & 0.6575 & 0.0066 & 0.7874 & 0.7941 & 0.1366 & 20.8 \\
\hline 5 & C & 0.6353 & 0.0128 & 0.6482 & 0.0018 & 0.7229 & 0.7247 & 0.0766 & 11.8 \\
\hline 5 & $D$ & 0.6354 & 0.0128 & 0.6483 & 0.0020 & 0.6979 & 0.6998 & 0.0516 & 8.0 \\
\hline 6 & A & 0.6191 & 0.0125 & 0.6316 & 0.0042 & 0.5919 & 0.5961 & -0.0355 & -5.6 \\
\hline 6 & B & 0.6495 & 0.0131 & 0.6626 & 0.0093 & 0.6379 & 0.6472 & -0.0154 & -2.3 \\
\hline 6 & $C$ & 0.6183 & 0.0125 & 0.6308 & 0.0000 & 0.0000 & 0.0000 & -0.6308 & -100.0 \\
\hline 6 & $\bar{D}$ & 0.6431 & 0.0130 & 0.6562 & 0.0027 & 0.6207 & 0.6234 & -0.0328 & -5.0 \\
\hline 7 & A & 0.6158 & 0.0125 & 0.6282 & 0.0024 & 0.7115 & 0.7139 & 0.0857 & 13.6 \\
\hline 7 & B & 0.6411 & 0.0130 & 0.6541 & 0.0027 & 0.6910 & 0.6937 & 0.0396 & 6.1 \\
\hline 7 & C & 0.6208 & 0.0126 & 0.6334 & 0.0009 & 0.7402 & 0.7411 & 0.1077 & 17.0 \\
\hline 7 & D & 0.6344 & 0.0128 & 0.6472 & 0.0014 & 0.8044 & 0.8057 & 0.1585 & 24.5 \\
\hline
\end{tabular}


Table 2.5 (Continued) Ash Balance of Hydrogenation Reactants and Products

\begin{tabular}{|c|c|c|c|c|c|c|c|c|c|}
\hline Run & Trial & $\begin{array}{l}\text { Ash In } \\
\text { Coal (g) }\end{array}$ & $\begin{array}{l}\text { Ash In } \\
\text { Solv. (g) }\end{array}$ & $\begin{array}{c}\text { Total Ash } \\
\text { In (g) }\end{array}$ & $\begin{array}{l}\text { Ash Out } \\
\text { Pitch (g) }\end{array}$ & $\begin{array}{c}\text { Ash Out THF } \\
\text { Ins. (g) }\end{array}$ & $\begin{array}{c}\text { Total Ash } \\
\text { Out }(g)\end{array}$ & $\begin{array}{l}\text { Out - In } \\
\text { (g) }\end{array}$ & $\begin{array}{c}\text { (Out-ln)/ln } \\
(\%)\end{array}$ \\
\hline 8 & A & 0.6414 & 0.0130 & 0.6544 & 0.0020 & 0.6952 & 0.6973 & 0.0429 & 6.6 \\
\hline 8 & B & 0.6168 & 0.0125 & 0.6293 & 0.0028 & 0.6671 & 0.6699 & 0.0406 & 6.4 \\
\hline 8 & C & 0.6341 & 0.0128 & 0.6469 & 0.0010 & 0.6503 & 0.6513 & 0.0043 & 0.7 \\
\hline 8 & D & 0.6190 & 0.0125 & 0.6315 & 0.0008 & 0.6169 & 0.6177 & -0.0138 & -2.2 \\
\hline 9 & A & 0.6451 & 0.0130 & 0.6581 & 0.0032 & 0.7030 & 0.7062 & 0.0481 & 7.3 \\
\hline 9 & B & 0.6252 & 0.0126 & 0.6379 & 0.0019 & 0.6871 & 0.6890 & 0.0511 & 8.0 \\
\hline 9 & C & 0.6592 & 0.0133 & 0.6726 & 0.0058 & 0.6539 & 0.6596 & -0.0129 & -1.9 \\
\hline 10 & A & 0.6176 & 0.0125 & 0.6301 & 0.0056 & 0.6780 & 0.6836 & 0.0535 & 8.5 \\
\hline 10 & $B$ & 0.6492 & 0.0131 & 0.6624 & 0.0101 & 0.7355 & 0.7456 & 0.0833 & 12.6 \\
\hline 10 & C & 0.6232 & 0.0126 & 0.6358 & 0.0055 & 0.6491 & 0.6546 & 0.0188 & 3.0 \\
\hline 10 & D & 0.6359 & 0.0129 & 0.6488 & 0.0066 & 0.6306 & 0.6372 & -0.0115 & -1.8 \\
\hline 11 & A & 0.8451 & 0.0000 & 0.8451 & 0.0077 & 0.9177 & 0.9254 & 0.0802 & 9.5 \\
\hline 11 & B & 0.8282 & 0.0000 & 0.8282 & 0.0068 & 0.9484 & 0.9552 & 0.1270 & 15.3 \\
\hline 11 & C & 0.8395 & 0.0000 & 0.8395 & 0.0022 & 0.9627 & 0.9649 & 0.1254 & 14.9 \\
\hline 11 & $\bar{D}$ & 0.8301 & 0.0000 & 0.8301 & 0.0171 & 0.8926 & 0.9097 & 0.0797 & 9.6 \\
\hline 12 & A & 1.0769 & 0.0000 & 1.0769 & 0.0016 & 1.0999 & 1.1015 & 0.0246 & 2.3 \\
\hline 12 & B & 1.0899 & 0.0000 & 1.0899 & 0.0047 & 1.0359 & 1.0406 & -0.0493 & -4.5 \\
\hline 12 & C & 1.1303 & 0.0000 & 1.1303 & 0.0015 & 1.1361 & 1.1376 & 0.0073 & 0.6 \\
\hline 12 & D & 1.1125 & 0.0000 & 1.1125 & 0.0033 & 1.1079 & 1.1112 & -0.0013 & -0.1 \\
\hline
\end{tabular}




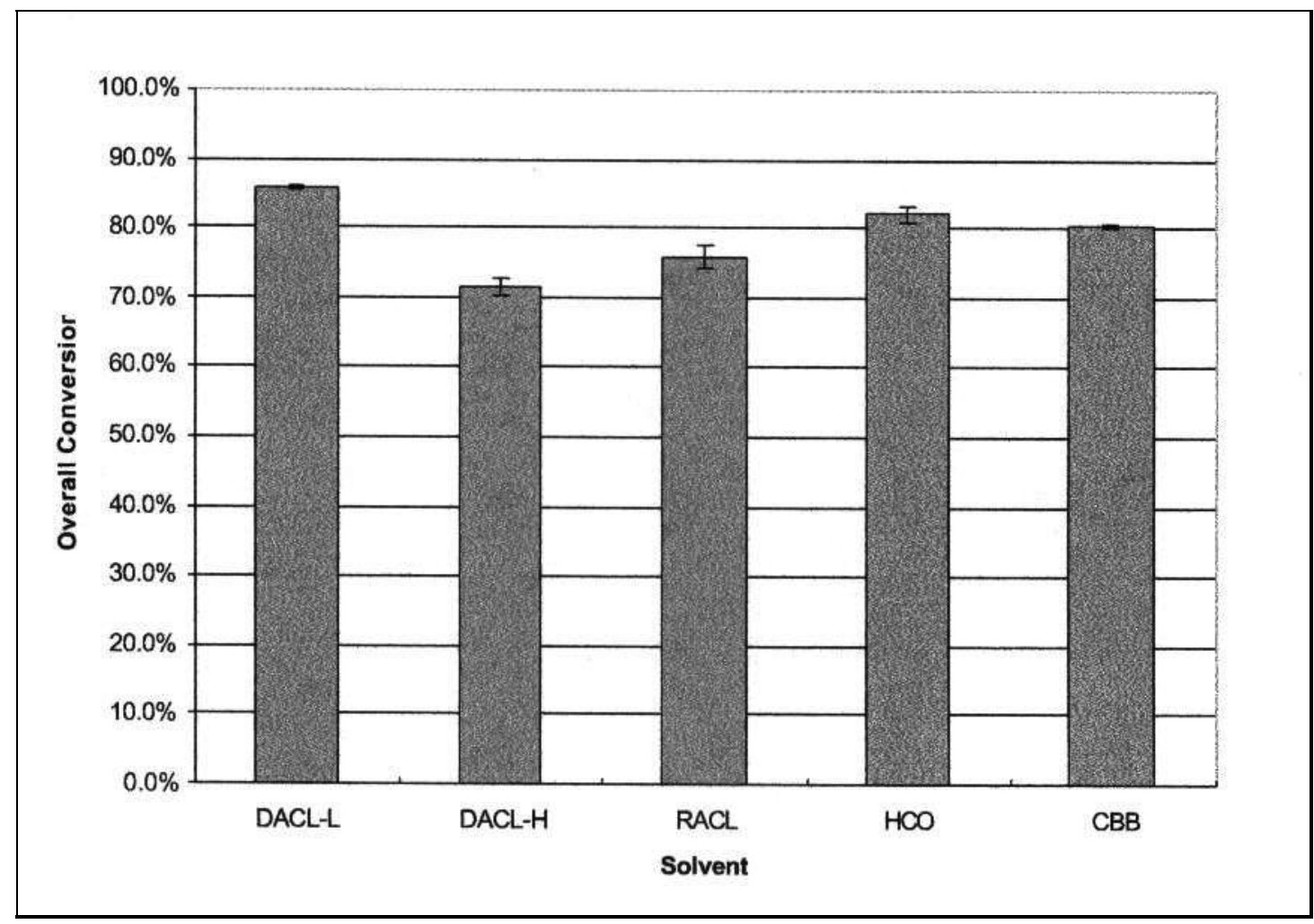

Figure 2.3 The effect of the hydrogenation solvent on the overall conversion to THF solubles at the reaction conditions of $400{ }^{\circ} \mathrm{C}, 1000 \mathrm{psig} \mathrm{H}_{2}$ (cold), 3/1 solvent-to-coal ratio, and 1 hour 


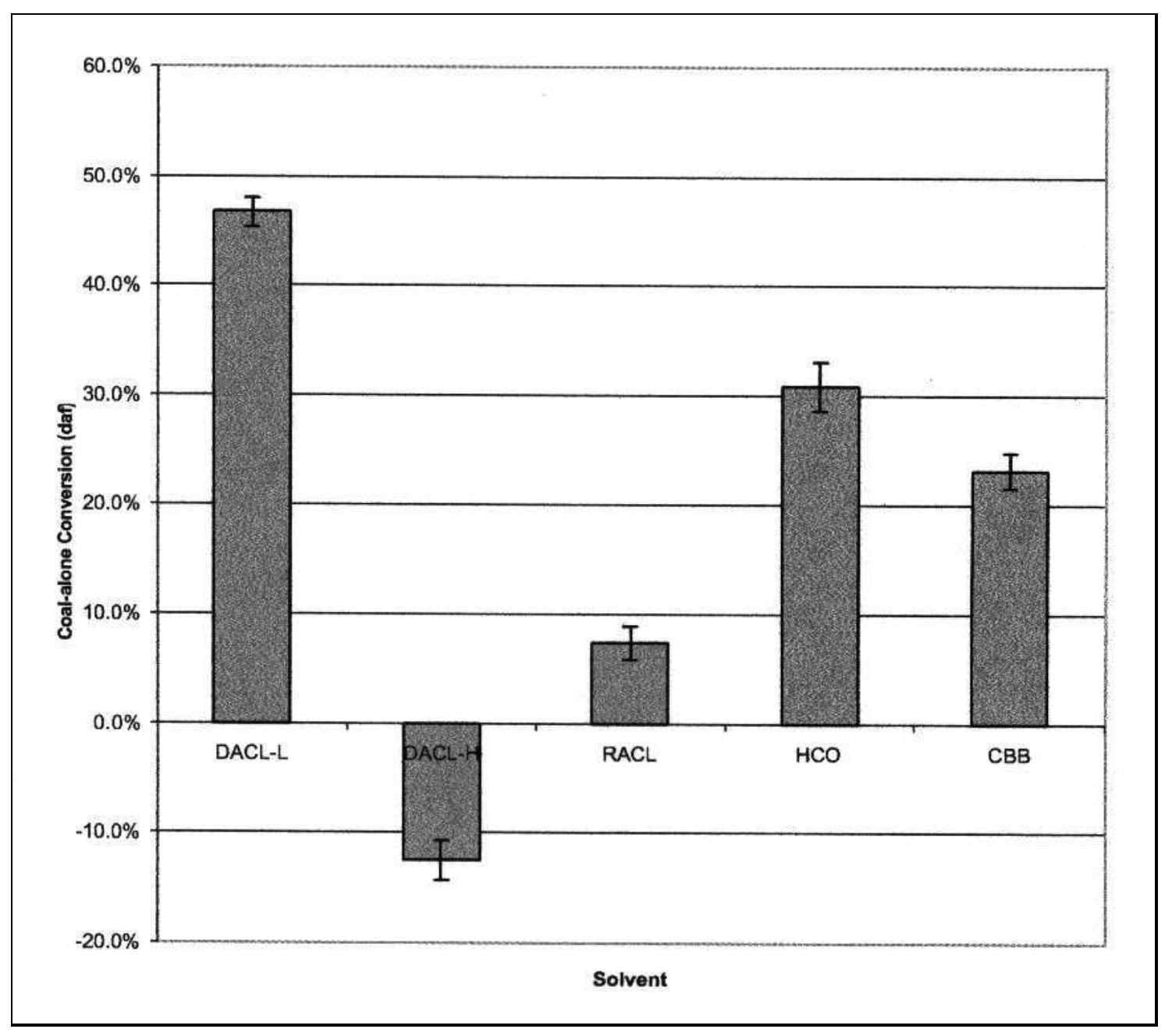

Figure 2.4 The effect of the hydrogenation solvent on the coal-alone conversion (daf basis) of coal to THF solubles at the reaction conditions of $400{ }^{\circ} \mathrm{C}, 1000$ psig $\mathrm{H}_{2}$ (cold), 3/1 solvent-to-coal ratio, and 1 hour

fraction of the hydrogenation products was derived from the solvent itself. Therefore, the effect of changing solvents on coal conversion was masked by the large amount of solvent that was being included in the overall conversion calculation. But nonetheless, the DACL-L did reasonably well by converting about $47 \%$ of the coal to THF-soluble products.

\subsubsection{Reaction Pressure}

The reactor pressures were recorded throughout all hydrogenation reactions in order to gain some insight into the role that the hydrogen atmosphere might play in the coal conversion. An empty reactor charged with hydrogen gas (1000 psig cold) only was also heated to the reaction temperature, and the pressure was recorded throughout the time scale of other hydrogenation reactions. The average reaction pressure recorded over time for each solvent is illustrated in Figure 2.5. The most glaring observation from Figure 2.5 is that after the 
pressure reached a maximum (between 1950 and 2150 psig depending on the solvent) due to the rapid heating, the pressure decreased with time for each run except for the empty reactor. There are two possible reasons for the observed effect. First, the reactors could have been leaking. However, the reactors were thoroughly checked and sealed before immersion in the sand bath. The reactors were also allowed to sit overnight following each reaction in order to insure that no slow leaks could be detected. If any reactors showed a decline in pressure after this period, the sample was discarded and thus not presented here. Therefore, leaks in the reactors were not the cause for such an effect. The second possible explanation was that the hydrogen gas was being consumed by the reactants. This being the most likely interpretation, the hydrogen could be transferred to two different components of the reaction. The hydrogen could have been consumed by hydrogenation of the solvent or by adding hydrogen to the coal such as described by the free radical mechanism of hydrogenation. At this time the exact mechanism of hydrogen consumption for these conditions cannot be determined.

The decrease in pressure during the hydrogenation reaction also indicates that the hydrogen gas is being consumed faster than gaseous products are being evolved. The second important observation from Figure 2.5 is the relative maximum pressure that each solvent achieved during the reactions. With the exception of RACL solvent, the relative height of the pressure curves decreases as the carbon content of the solvent increases. This was expected since as the carbon content of the solvent increases so does the concentration of higher molecular weight species in the solvent. These higher molecular weight species would not be as volatile under the present reaction conditions, thus corresponding to lower reactor pressures.

Finally, it is important to notice the pressure of the reactor system once the reaction has been quenched by immersion in a water bath. The sharp decrease in pressure that occurred at sixty minutes is due to this quenching process. Once the pressure drop ceased (usually by the 90 minute mark) the reactor could be considered at room temperature. It is significant to note that for each reaction system illustrated in Figure 2.5, the final pressure of the system (720 to 850 psig depending on the solvent) was less than that of the initial pressure (1000 psig $\mathrm{H}_{2}$ cold). This again may indicate the consumption of hydrogen during the hydrogenation reaction.

\subsubsection{Product Distillation}

Once the hydrogenation reaction products were separated into THF soluble and insoluble fractions and the THF was removed from the soluble fraction, the soluble fraction was further split into two fractions, the light distillate (or recycle solvent) and the heavy distillation residue (or pitch). This separation was achieved by the vacuum distillation described in Section 2.2.9. Each trial of every run was distilled to a different extent in order to collect a different amount of recycle solvent. This was done to gain some insight into the effect that the distillation process would have on the resultant pitch softening point and other characteristics such as coking value, ash content, and optical texture. 


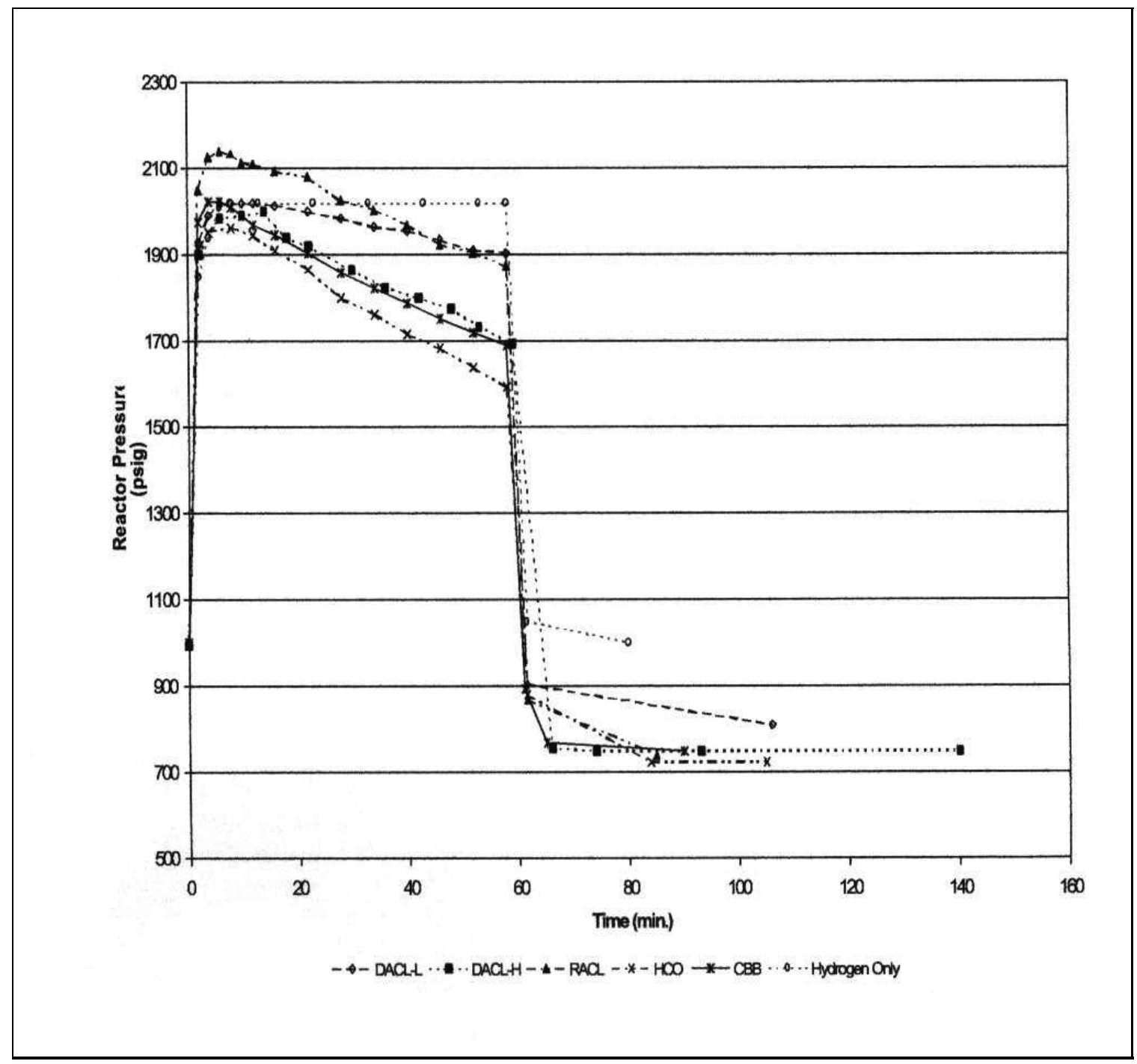

Figure 2.5 Reactor pressures during coal hydrogenation reactions with coal-derived solvents at reaction conditions of $400{ }^{\circ} \mathrm{C}$, $1000 \mathrm{psig} \mathrm{H}_{2}$ (cold), 3/1 solventto-coal ratio, and 1 hour

Figure 2.6 shows the effect that the extent of distillation has on the softening point of the pitch resulting from the hydrogenation reactions. This figure shows a linear relationship between the amount of feed recovered as pitch and the softening point of the resultant pitch. Using this relationship, a pitch can be tailored to a specific softening point by controlling the distillation step of production. The relative amounts of pitch recovered for the products made from the three Antaeus coal liquids show a predictable relationship. The light material (DACL-L) required the most distillation to achieve a softening point of $120^{\circ} \mathrm{C}$ or higher. 
The heavy fraction (DACL-H) required the least amount of distillation while the original coal liquid (RACL) fell in between and nearly in

the middle of these two lines. This was not unexpected since the split of DACL-L and DACL-H from RACL was approximately $50 \%$ by weight.

The two interesting trends shown in Figure 2.6 are those for $\mathrm{HCO}$ and $\mathrm{CBB}$. According to the trends shown by the Antaeus coal liquids, the heavier materials should be shifted to the right of this graph. The starting materials for these two sets of reactions were clearly higher in heavy material than that of DACL-L. However, both of the Koppers Industries, Inc. liquids fell to the left (required more distillation) than that of DACL-L. This phenomenon can be explained by examining the coal-alone conversion exhibited when each of these solvents was employed. DACL-L had a significantly higher coal-alone conversion (approximately $47 \%$ ) than that of $\mathrm{HCO}$ and CBB (approximately $31 \%$ and $23 \%$ respectively). Therefore, the products recovered from reactions performed with DACL-L should have more heavy components (converted from coal) than those reactions using $\mathrm{HCO}$ and CBB. This would lead to the phenomenon of the HCO and CBB distillation trends shown in Figure 2.6 being to the left of the DACL-L linear representation.

\subsubsection{Hydrogenation Products}

Once the distillation step has occurred, the hydrogenation products have now been partitioned into three different fractions: (1) THF insolubles, (2) pitch, and (3) recycle solvent. The THF insolubles and recycle solvents were considered to be by-products of pitch production and, hence, were not thoroughly characterized. The ash content of the THF insolubles was determined so that an ash balance (Section 2.4.2) could be calculated, and an elemental analysis was performed on several of the insoluble and recycle solvent fractions. The remainder of this section is focused on characterizing and comparing the pitch produced by hydrogenation with the different coal-derived solvents. In order to compare the pitches produced from the separate solvents, pitches of approximately the same softening point $\left(\mathrm{T}_{\mathrm{sp}}\right.$ $\approx 120{ }^{\circ} \mathrm{C}$ ) were derived and characterized. 


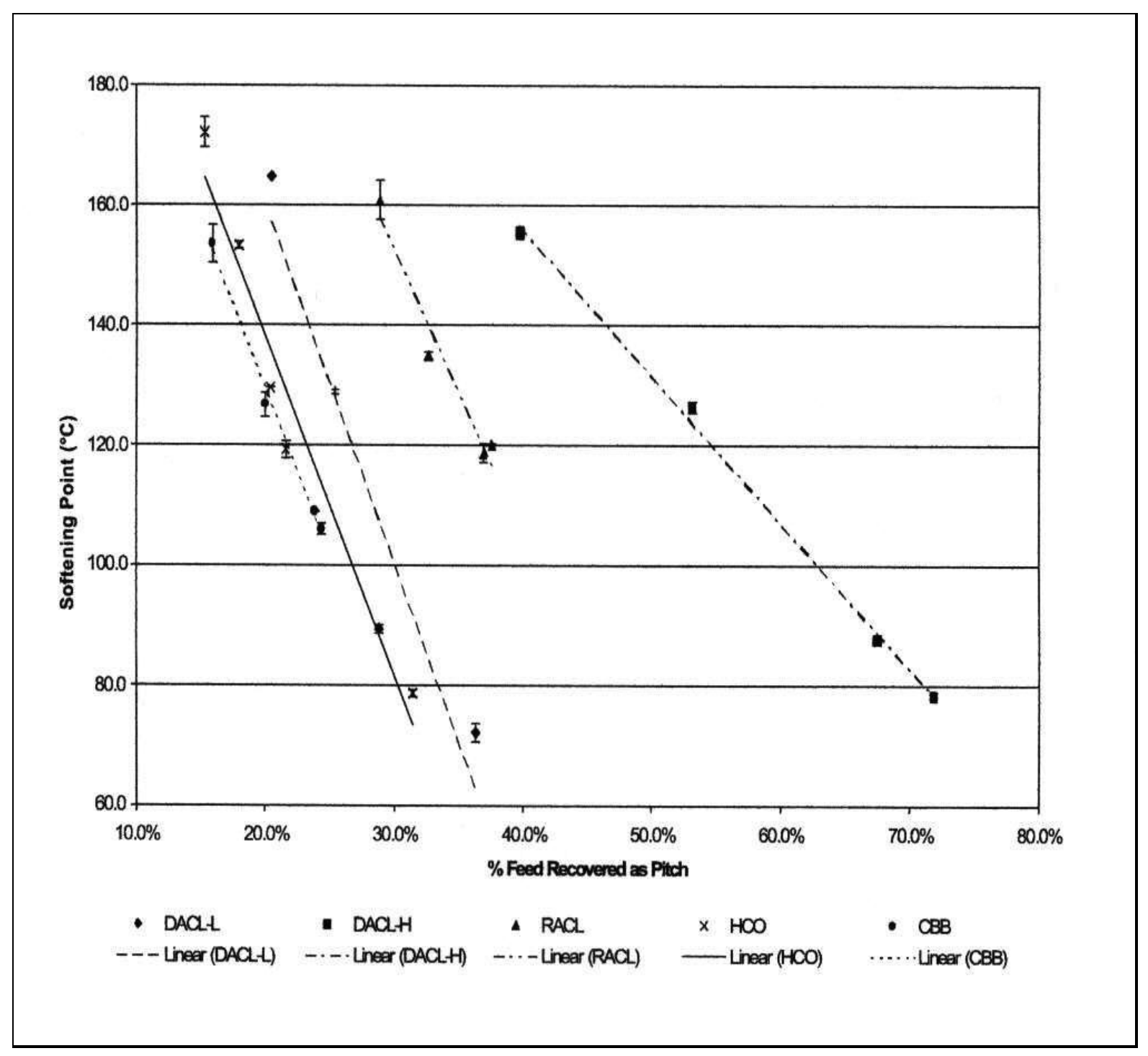

Figure 2.6 Effect of distillation on the softening point of the pitch resulting from hydrogenation reactions with coal-derived solvents at reaction conditions of $400{ }^{\circ} \mathrm{C}, 1000 \mathrm{psig} \mathrm{H}_{2}$ (cold), 3/1 solvent-to-coal ratio, and 1 hour

The product distribution of the reaction products with a softening point of about $120{ }^{\circ} \mathrm{C}$ depends on the particular solvent. Figure 2.7 illustrates the product distribution for the hydrogenation reactions using the five coal-derived solvents. The product distribution from the reactions using DACL-L, HCO, and CBB all appear rather similar. The dominant fraction for each reaction was the recycle solvent, with the pitch fraction being the next highest. DACL-H reactions exhibit a very different profile in Figure 2.7. The majority of the product was converted to pitch, however, the coal conversion for these reactions was the lowest (even negative for the coal only conversion). This phenomenon is created by the 
physical properties of the original solvent. DACL-H at room temperature is solid pitch-like material with a softening point greater than $100^{\circ} \mathrm{C}$. Therefore, after reaction, only the light material created during hydrogenation needs to be removed to get the softening point to 120 ${ }^{\circ} \mathrm{C}$, and since the conversion is so low, very little light material was produced causing the high pitch production with low recycle solvent production.

It is also interesting to note that according to the above discussion and Figure 2.7 it is apparent that there is a trade-off between the amount of pitch produced and the amount of recycle solvent recovered depending on the solvent employed for hydrogenation. A large amount of pitch means more final pitch product, however, a low amount of recycle solvent means that the process may not be able to sustain itself. Therefore, a balance between the amount of pitch and recycle solvent needs to be struck. However, this balance may not be an equal one depending on the amount of recycle solvent required to perform subsequent reactions. This would depend on the optimum solvent-to-coal ratio which is discussed in a later section.

\subsubsection{Ash Content}

The ash content of the pitch and THF insoluble fractions was determined according to the ASTM method outlined in Section 2.3.3. For most carbon-product precursors, ash is considered to be an impurity and as such low values are required for the product pitches. The amount of relative error in determining the ash content for the insoluble fractions, which contain most of the ash, is small, $\pm 2 \%$. However, the pitch fractions contain a very small amount of ash (usually less than $0.1 \%$ by weight) leading to a much higher relative error of $\pm 18 \%$ for the ash determinations. Several of the recycle solvents were tested for ash, and each was found to contain no ash. This is expected since the recycle solvent is the distillate portion of the hydrogenation products. Therefore, any ash that may have been transferred into the THF soluble portion of the hydrogenation products would be concentrated in the pitch sample. 


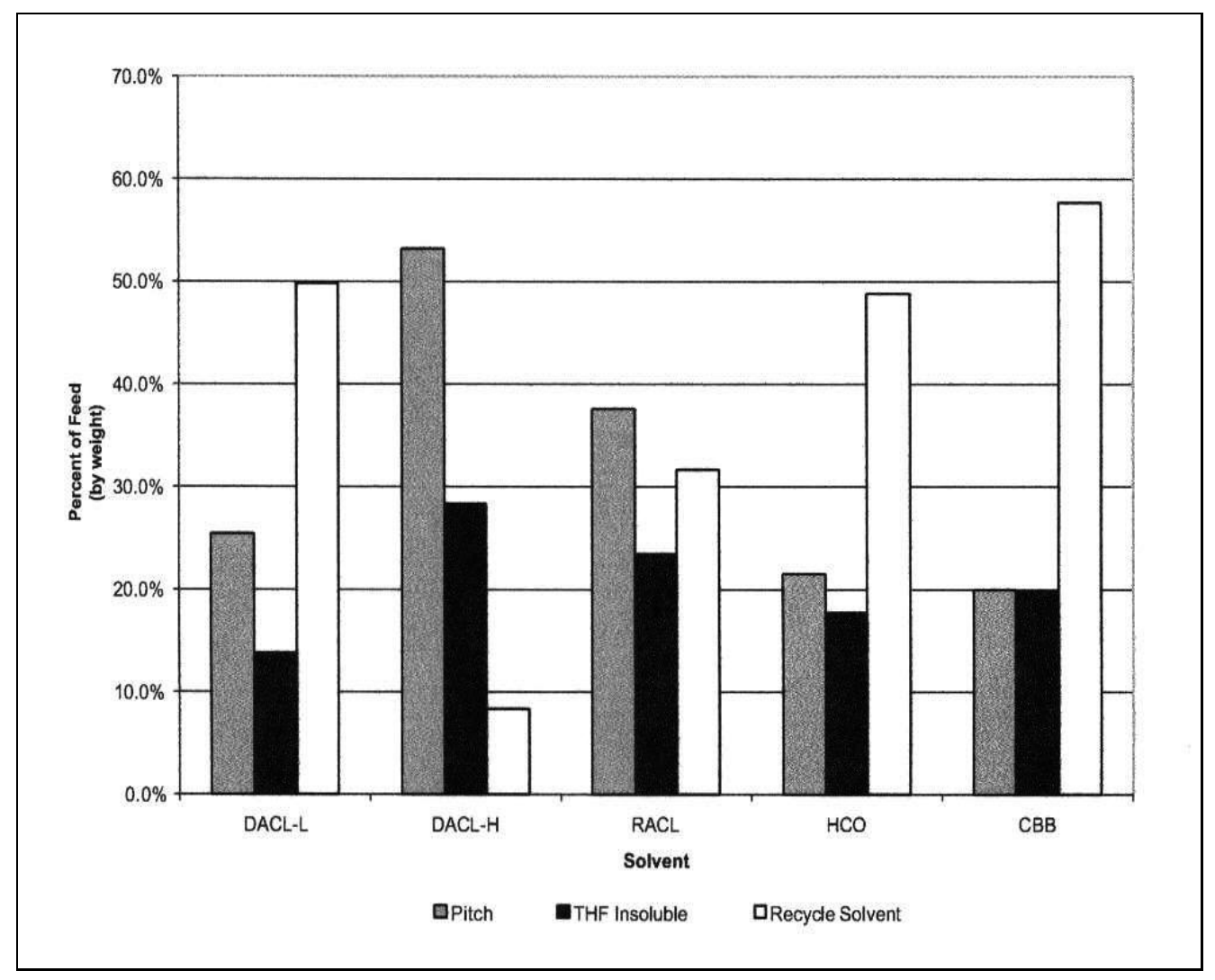

Figure 2.7 Effect of solvent choice on the hydrogenation product distribution with reaction conditions of $400{ }^{\circ} \mathrm{C}, 1000$ psig $\mathrm{H}_{2}$ (cold), 3/1 solvent-to-coal ratio, and 1 hour for a pitch with approximately $120^{\circ} \mathrm{C}$ softening point

Table 2.6 Softening Point of Pitch and Ash Content of Pitch and THF Insoluble Fractions of Hydrogenation Products.

\begin{tabular}{|c|c|c|c|}
\hline Solvent & $\begin{array}{c}\text { Pitch } \\
\text { Softening } \\
\text { Point }\left({ }^{\circ} \mathrm{C}\right)\end{array}$ & $\begin{array}{c}\text { \% Ash in } \\
\text { Pitch }\end{array}$ & $\begin{array}{c}\text { \% Ash in } \\
\text { THF Insol. }\end{array}$ \\
\hline DACL-L & 129.0 & $0.110 \%$ & $11.55 \%$ \\
\hline DACL-H & 126.5 & $0.025 \%$ & $7.11 \%$ \\
\hline RACL & 120.2 & $0.009 \%$ & $7.79 \%$ \\
\hline HCO & 119.4 & $0.919 \%$ & $10.63 \%$ \\
\hline CBB & 126.9 & $0.093 \%$ & $11.10 \%$ \\
\hline
\end{tabular}


The ash contents of the pitch and insoluble fractions of the hydrogenation products are presented in Table 2.6 for each different solvent. The percent ash in all of the pitch products is at or less than $0.1 \%$ signifying that they are all very pure products. The ash content of the insoluble fraction has a wide range depending on the type of solvent that was used. This can be related to the solvent's ability to convert the organic material of the coal into soluble material. The solvents showing the higher coal conversions (Figure 2.4) also have the highest ash contents for the THF insolubles. As more coal is converted to soluble material the percentage of ash in the unconverted material (THF insoluble) would rise since it is based on the total organic material in the residue and not the coal. Reactions performed with DACL-L also produced the pitch with the highest ash content, but this could be due to the error in the ash analysis.

\subsubsection{Coke Yield}

The coking value gives an indication of the amount of non-volatile matter in the pitch sample. The coke yield was determined according to the procedure described in Section 2.3.2. This characterization technique typically has a relative error of $\pm 2 \%$ so it is an accurate measurement. The coke yields of the pitches with a softening point of approximately $120^{\circ} \mathrm{C}$ for each solvent-coal reaction are detailed in Figure 2.8. Each of these coke yields are in the range of 43.5 to $48 \%$ by weight, which is slightly below the desired value of commercial pitches $(50-60 \%)$. However, the difference in coke yields when compared by the various solvents is insignificant when factoring in the relative error of the test.

There are two possible ways of increasing the coke yield of the hydrogenation pitches. First, the desired softening point of the pitch could be increased. This would eliminate more of the light volatile compounds that are relinquished during the coking. The second possible way of increasing the coke yield of the pitch is to lower the solvent-to-coal ratio of the hydrogenation reactions assuming the coal conversion is the same at a lower solvent-to-coal ratio. This would reduce the amount of solvent in the final pitch product, thus increasing the coking value. The results of lowering the solvent-to-coal ratio are discussed in a later section. The effect of raising the softening point on the coke yield of the resultant pitch is illustrated by Figure 2.9. For all of the pitches shown, there is a linear dependence of coke yield on the pitch softening point. Furthermore, all of the pitches are in the same basic range on the graph except DACL-L derived pitches, which are shifted slightly to the right. This suggests that the pitch made from coal and DACL-L 


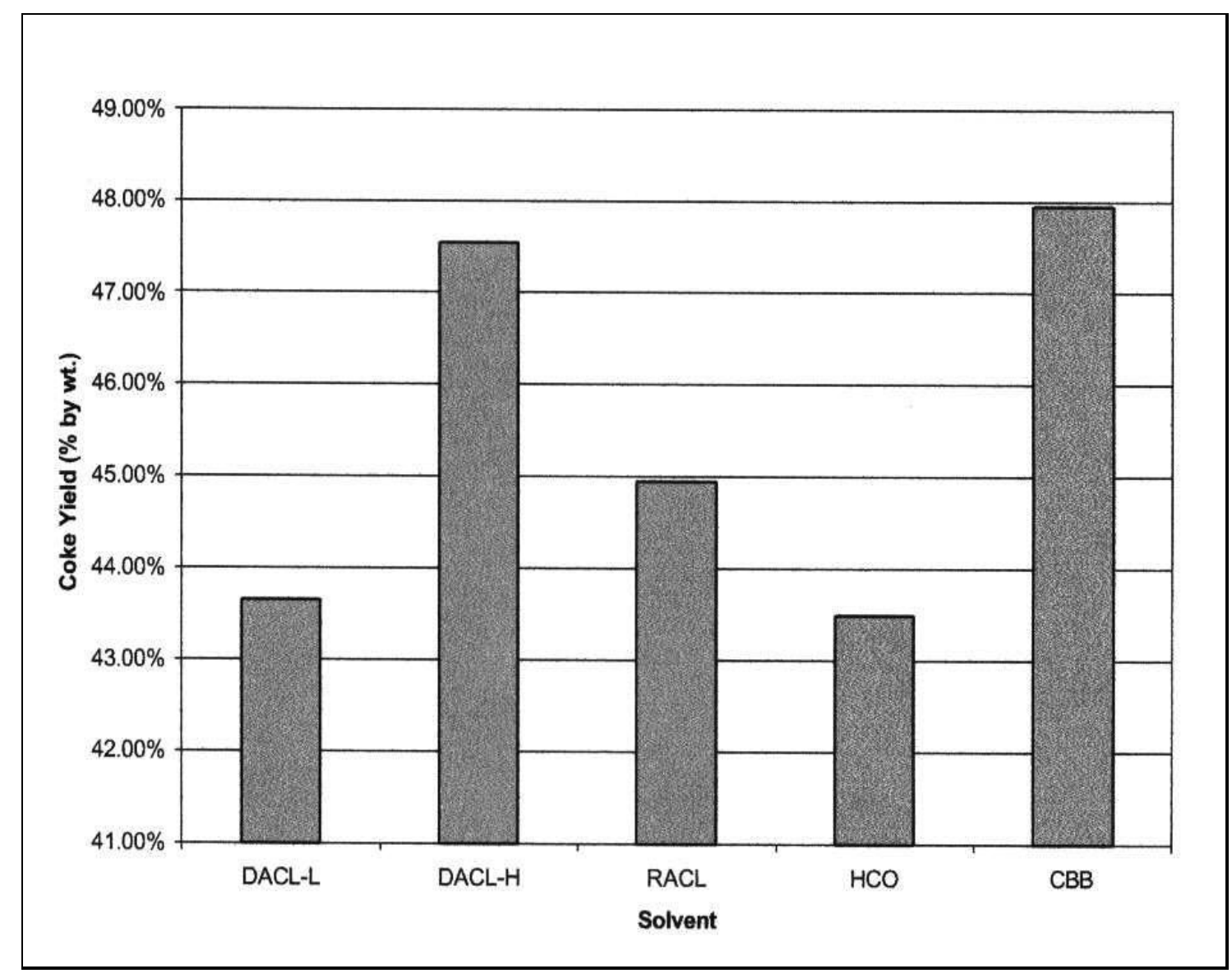

Figure 2.8 Effect of hydrogenation solvent on the coke yield of the pitches resulting from hydrogenation reactions with the conditions of $400{ }^{\circ} \mathrm{C}, 1000 \mathrm{psig}$ $\mathrm{H}_{2}$ (cold), 3/1 solvent-to-coal ratio, and 1 hour. Pitch softening point is around $120{ }^{\circ} \mathrm{C}$. 


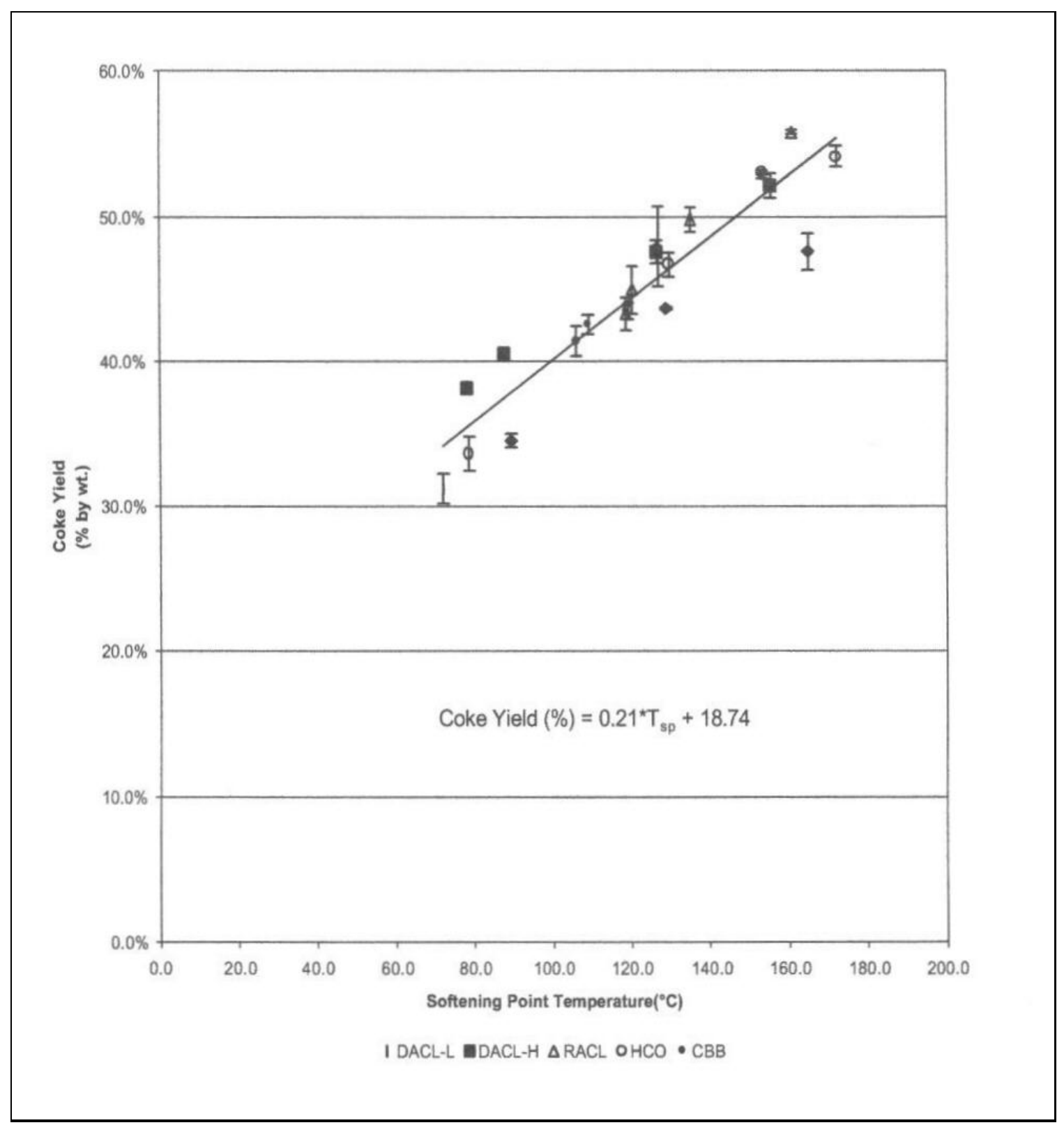

Figure 2.9 Effect of softening point on the coke yield of pitches made from hydrogenation reactions using the five different coal-derived solvents under reaction conditions of $400{ }^{\circ} \mathrm{C}, 1000 \mathrm{psig} \mathrm{H}_{2}$ (cold), 3/1 solvent-tocoal ratio, and 1 hour

contains on average more volatile components, thus requiring greater distillation (leading to an increase in softening point) to increase the coke yield. In order to achieve a pitch with a coke yield of greater than $50 \%$ by weight, Figure 2.9 shows that the softening point of the pitch needs to be at or above approximately $140{ }^{\circ} \mathrm{C}$. 


\subsubsection{Optical Texture}

Once the coke samples were weighed and the coke yield was calculated, the coke sample was embedded in epoxy and polished for observation under the polarized-light microscope. This technique allowed for determination of the optical texture of the coke, which is extremely important in determining the end use of the carbon product precursor. The optical texture was determined according the procedure described in Section 2.3.4, and photographs were taken of all coke samples. Figure 2.10 shows cokes produced from the various pitches; the softening point of the pitches was approximately $120^{\circ} \mathrm{C}$. All reaction conditions for these pitches were the same except for the choice of solvent.

Figure 2.10 shows that the optical texture for each of the cokes produced from hydrogenation reactions of coal and a coal-derived solvent were anisotropic. The major difference observed when comparing the solvents is in the domain size of the anisotropic cokes. The domains produced by using DACL-H and RACL (Figure 2.10 B and C, respectively) seem to be similar in size and are also fairly uniform throughout the sample. The cokes produced from $\mathrm{HCO}$ and $\mathrm{CBB}$ solvents (Figure 2.10 D and E, respectively) have areas of very large domains interspersed with areas of slightly smaller domains, similar to that of the cokes produced with DACL-H and RACL. The type of texture determines the possible end uses for the carbon product precursors. It is known that the anisotropic coke is required for the production of graphite electrodes, while the isotropic coke is more suited to the production of nuclear graphite which requires a high purity and uniform dimensional stability.

\subsubsection{Elemental Analysis}

An elemental analysis was performed on a select group of pitches and their corresponding THF insoluble and recycle solvent fractions. The select pitches were again ones with a softening point of approximately $120^{\circ} \mathrm{C}$ and from each different solvent. The elemental analysis was used to determine the amount of carbon, hydrogen, nitrogen, and sulfur in the sample according to the procedure outline in Section 2.3.5. The results of the elemental analysis for the carbon and hydrogen percentage in the samples are very accurate with relative errors of $\pm 2 \%$ for carbon and $\pm 5 \%$ for the hydrogen content. The error involved in determining the nitrogen and sulfur content in the samples is significantly larger due to the fact that the samples contain smaller amounts of these two elements compared to the carbon content. The relative error involved in determining the nitrogen and sulfur content is on the order of $\pm 20 \%$. Therefore, further discussion on the elemental content of the hydrogenation reactants and products will only concern the carbon and hydrogen content. 


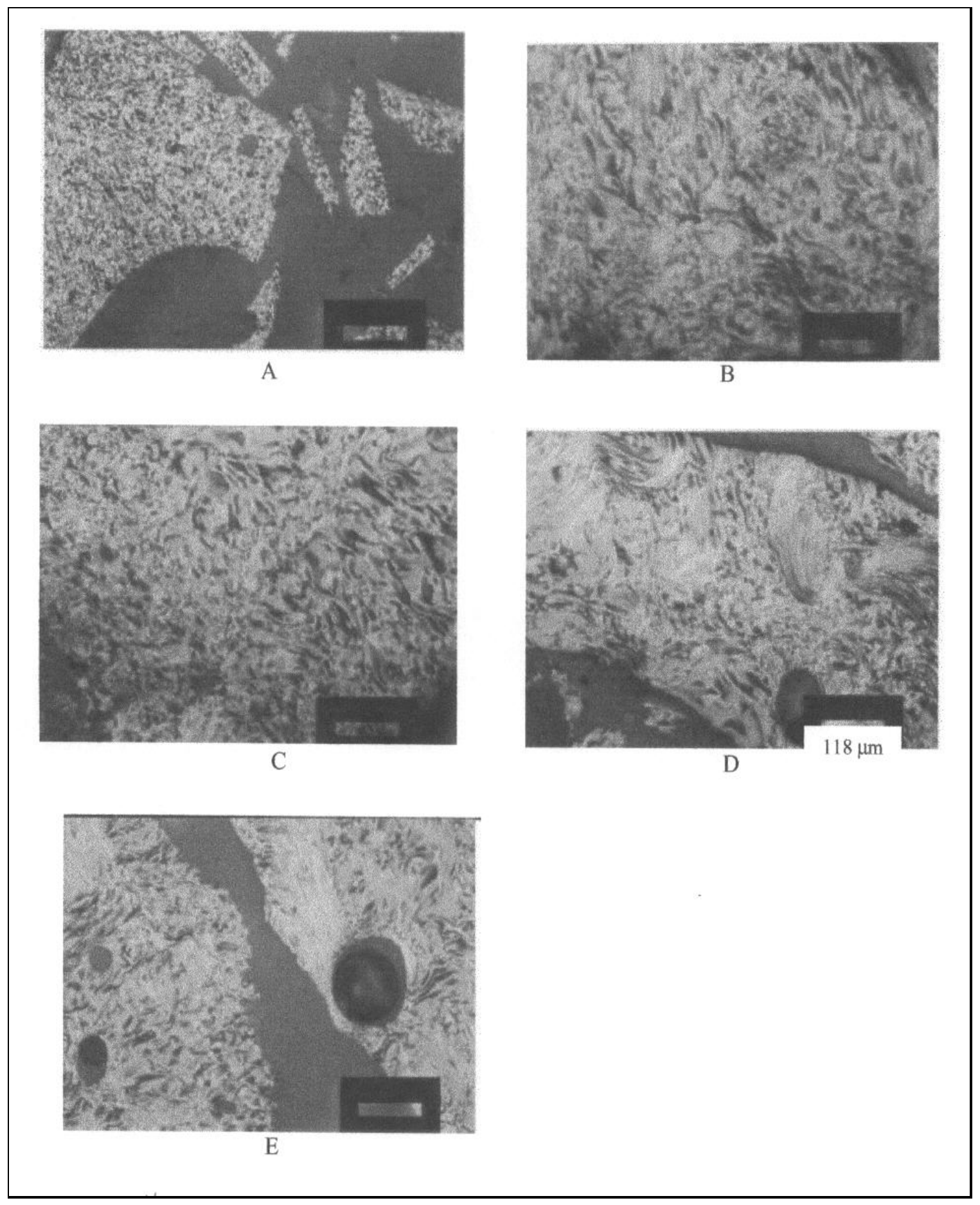

Figure 2.10 Optical micrographs of cokes produced from pitches (with softening points around $120^{\circ} \mathrm{C}$ ) using different solvents during hydrogenation reactions with conditions of $400{ }^{\circ} \mathrm{C}, 1000$ psig $\mathrm{H}_{2}$ (cold), 3/1 solvent-tocoal ratio, and 1 hour and solvents A) DACL-L, B) DACL-H, C) RACL, D) HCO, and E) CBB 
The elemental composition and hydrogen-to-carbon atomic ratios of the selected reaction products are shown in Table 2.7. The reaction conditions for each run can be seen in Table 2.3. For each set of reaction products, some general trends can be observed. The hydrogento-carbon ratio increases for each hydrogenation run from the THF insolubles to the pitch fraction up to the recycle solvent. This is the expected result since the recycle solvent is the lightest component of the reaction products corresponding to the highest hydrogen-to-carbon ratio. Also, the hydrogen-to-carbon atomic ratios for the recycle solvents have a higher value than that of the original solvent employed in the reaction. This indicates that some lighter material was being produced during the hydrogenation reaction. The carbon content of the product pitches are similar at a value of about $90 \%$ by weight. The THF insoluble fraction also does not show a significant difference in carbon $(\approx 81 \%)$ and hydrogen $(\approx 4 \%)$ content when comparing the different solvents used. However, the recovered recycle solvent does show a significant difference from one sample to another. The carbon content of the recycle solvents recovered from the reactions using $\mathrm{HCO}$ and $\mathrm{CBB}$ (93.9\% and 90.7\%, respectively) are higher than those produced using the other solvents (less than $85 \%$ ). This can be attributed to the fact that the original solvents, $\mathrm{HCO}$ and $\mathrm{CBB}$, have a higher carbon content than the other solvents.

The elemental analysis was also used to perform a carbon and hydrogen balance for each of the elected hydrogenation reactions. The results of the elemental balances can be seen in Table 2.8 and Table 2.9 for carbon and hydrogen, respectively. The carbon balance shows a negative balance for all of the selected runs. This is consistent with the negative mass balance that was observed during each of these runs. Since carbon is by far the most abundant element in all of the reaction species, any loss of mass would correspond to a significant loss in elemental carbon causing the observed negative carbon balances. The hydrogen balances shown in Table 2.9 also show a negative hydrogen balance for each of the selected runs. This also corresponds to a negative mass balance. However, a positive hydrogen balance could have been expected from examining the transient reactor pressure for each reaction (see Figure 2.5). It showed that hydrogen was being consumed by the hydrogenation reactants throughout the thermal treatment process. Since hydrogen was being consumed, one might expect an increase in the total hydrogen out of the hydrogenation reactions. However, by examining the different causes for error in the mass balance calculation, the explanation of a negative hydrogen balance becomes apparent. The two major causes for loss of mass throughout these reactions were the fact that the product gas was not quantified and the loss of light material during THF removal and vacuum distillation. Both of these mass losses involve the loss of the lightest products which are produced by the hydrogenation reactions. These light materials would have been the significantly more hydrogen-rich products of the reactions. Because these materials are the ones that are unaccounted for in the mass balance calculation and elemental analysis, a negative hydrogen balance unfortunately occurs. 
Table 2.7 Elemental Composition of Select Hydrogenation Products

\begin{tabular}{|c|c|c|c|c|c|}
\hline \multirow[b]{2}{*}{ Run } & \multirow[b]{2}{*}{ Element } & \multirow[b]{2}{*}{$\begin{array}{l}\text { Original } \\
\text { Solvent } \\
\end{array}$} & \multicolumn{3}{|c|}{ Product Fraction } \\
\hline & & & Pitch & $\begin{array}{c}\text { THF } \\
\text { Insoluble }\end{array}$ & $\begin{array}{l}\text { Recycle } \\
\text { Solvent }\end{array}$ \\
\hline \multirow{5}{*}{$1 \mathrm{~A}$} & $\mathrm{C}$ & $80.51 \%$ & $86.80 \%$ & $82.52 \%$ & $82.62 \%$ \\
\hline & $\mathrm{H}$ & $7.74 \%$ & $6.16 \%$ & $4.18 \%$ & $8.21 \%$ \\
\hline & $\mathrm{N}$ & $0.94 \%$ & $1.22 \%$ & $1.53 \%$ & $0.87 \%$ \\
\hline & $S$ & $0.61 \%$ & $0.67 \%$ & $0.91 \%$ & $0.19 \%$ \\
\hline & $\begin{array}{c}\text { H/C Atomic } \\
\text { Ratio } \\
\end{array}$ & 1.15 & 0.85 & 0.61 & 1.19 \\
\hline \multirow{5}{*}{$2 \mathrm{C}$} & $\mathrm{C}$ & $88.51 \%$ & $89.70 \%$ & $83.08 \%$ & $85.60 \%$ \\
\hline & $\mathrm{H}$ & $5.72 \%$ & $5.62 \%$ & $4.15 \%$ & $7.25 \%$ \\
\hline & $\mathrm{N}$ & $1.25 \%$ & $1.68 \%$ & $2.02 \%$ & $1.48 \%$ \\
\hline & $s$ & $0.76 \%$ & $0.66 \%$ & $0.85 \%$ & $0.57 \%$ \\
\hline & $\begin{array}{c}\text { H/C Atomic } \\
\text { Ratio } \\
\end{array}$ & 0.78 & 0.75 & 0.60 & 1.02 \\
\hline \multirow{5}{*}{ 3B } & $\mathrm{C}$ & $86.67 \%$ & $90.53 \%$ & $80.72 \%$ & $81.86 \%$ \\
\hline & $\mathrm{H}$ & $6.76 \%$ & $5.83 \%$ & $4.55 \%$ & $8.44 \%$ \\
\hline & $\mathrm{N}$ & $1.22 \%$ & $1.29 \%$ & $2.19 \%$ & $1.87 \%$ \\
\hline & $S$ & $0.72 \%$ & $0.73 \%$ & $0.92 \%$ & $0.67 \%$ \\
\hline & $\begin{array}{c}\text { H/C Atomic } \\
\text { Ratio }\end{array}$ & 0.94 & 0.77 & 0.68 & 1.24 \\
\hline \multirow{5}{*}{$4 \mathrm{C}$} & $\mathrm{C}$ & $93.41 \%$ & $91.16 \%$ & $79.02 \%$ & $93.90 \%$ \\
\hline & $\mathrm{H}$ & $5.14 \%$ & $5.31 \%$ & $3.96 \%$ & $5.23 \%$ \\
\hline & $\mathrm{N}$ & $0.88 \%$ & $1.45 \%$ & $1.42 \%$ & $0.93 \%$ \\
\hline & $S$ & $0.55 \%$ & $0.71 \%$ & $0.96 \%$ & $0.48 \%$ \\
\hline & $\begin{array}{c}\mathrm{H} / \mathrm{C} \text { Atomic } \\
\text { Ratio }\end{array}$ & 0.66 & 0.70 & 0.60 & 0.67 \\
\hline \multirow{5}{*}{$5 B$} & $\mathrm{C}$ & $91.85 \%$ & $89.75 \%$ & $79.17 \%$ & $90.73 \%$ \\
\hline & $\mathrm{H}$ & $5.51 \%$ & $5.14 \%$ & $3.97 \%$ & $5.79 \%$ \\
\hline & $\mathrm{N}$ & $0.81 \%$ & $1.15 \%$ & $0.86 \%$ & $0.89 \%$ \\
\hline & $\mathrm{s}$ & $0.67 \%$ & $0.63 \%$ & $0.94 \%$ & $0.69 \%$ \\
\hline & $\begin{array}{l}\text { H/C Atomic } \\
\text { Ratio }\end{array}$ & 0.72 & 0.69 & 0.60 & 0.77 \\
\hline
\end{tabular}


Table 2.8 Elemental Carbon Balance for Hydrogenation Reactants and Products

\begin{tabular}{|c|c|c|c|c|c|c|c|c|c|c|}
\hline Run & \begin{tabular}{|l|} 
Trial \\
\end{tabular} & $\begin{array}{l}\text { Carbon } \\
\text { In Coal } \\
(\mathrm{g})\end{array}$ & $\begin{array}{l}\text { Carbon In } \\
\text { Solv. (g) }\end{array}$ & \begin{tabular}{|c|} 
Total \\
Carbon In \\
(g)
\end{tabular} & $\begin{array}{l}\text { Carbon } \\
\text { Out Pitch } \\
\text { (g) }\end{array}$ & \begin{tabular}{|l|} 
Carbon \\
Out THF \\
Ins. (g)
\end{tabular} & $\begin{array}{l}\text { Carbon } \\
\text { Out Rec. } \\
\text { Solv. (g) }\end{array}$ & $\begin{array}{l}\text { Total } \\
\text { Carbon } \\
\text { Out (g) }\end{array}$ & $\begin{array}{l}\text { Out - In } \\
\text { (g) }\end{array}$ & $\begin{array}{l}\text { (Out- } \\
\text { In)//n } \\
(\%)\end{array}$ \\
\hline 1 & A & 6.8381 & 20.2105 & 27.0486 & 7.3812 & 3.8101 & 13.7776 & 24.9690 & -2.0796 & -7.7 \\
\hline 2 & C & 4.8960 & 15.9325 & 20.8285 & 11.4446 & 5.6568 & 1.7034 & 18.8048 & -2.0237 & -9.7 \\
\hline 3 & B & 6.2449 & 19.8992 & 26.1441 & 10.4227 & 5.8113 & 7.9482 & 24.1822 & $-1,9619$ & -7.5 \\
\hline 4 & C & 7.1860 & 24.7083 & 31.8944 & 6.9261 & 4.9389 & 16.1406 & 28.0056 & -3.8888 & -12.2 \\
\hline 5 & B & 7.3079 & 24.6799 & 31.9878 & 6.4201 & 5.6164 & 18.7533 & 30.7898 & -1.1980 & -3.7 \\
\hline
\end{tabular}

Table 2.9 Elemental Hydrogen Balance for Hydrogenation Reactants and Products

\begin{tabular}{|c|c|c|c|c|c|c|c|c|c|c|}
\hline Run & \begin{tabular}{|l|} 
Trial \\
\end{tabular} & $\begin{array}{c}\text { Hydrogen in } \\
\text { Coal }(\mathrm{g})\end{array}$ & $\begin{array}{c}\text { Hydrogen } \\
\text { in Solv. } \\
\text { (g) }\end{array}$ & \begin{tabular}{|c} 
Total \\
Hydrogen \\
In (g)
\end{tabular} & $\begin{array}{l}\text { Hydrogen } \\
\text { Out Pitch } \\
\text { (g) }\end{array}$ & \begin{tabular}{|l} 
Hydrogen \\
Out THF \\
Ins. (g)
\end{tabular} & \begin{tabular}{|l} 
Hydrogen \\
Out Rec. \\
Solv. (g) \\
\end{tabular} & \begin{tabular}{|c|} 
Total \\
Hydrogen \\
Out (g) \\
\end{tabular} & $\begin{array}{c}\text { Out - In } \\
\text { (g) }\end{array}$ & $\begin{array}{l}\text { (Out- } \\
\text { In)/n } \\
(\%)\end{array}$ \\
\hline 1 & A & 0.3825 & 1.9426 & 2.3251 & 0.5236 & 0.1928 & 1.3695 & 2.0859 & $|-0.2391|$ & $\mid-10.3$ \\
\hline 2 & C & 0.2739 & 1.0299 & 1.3038 & 0.7168 & 0.2826 & 0.1442 & 1.1436 & -0.1602 & $\mid-12.3$ \\
\hline 3 & B & 0.3493 & 1.5525 & 1.9019 & 0.6717 & 0.3274 & 0.8198 & 1.8190 & -0.0829 & -4.4 \\
\hline 4 & C & 0.4020 & 1.3614 & 1.7633 & 0.4031 & 0.2477 & 0.9109 & 1.5617 & $\mid-0.2017$ & $\mid-11.4$ \\
\hline 5 & B & 0.4088 & 1.4804 & 1.8892 & 0.3676 & 0.2817 & 1.1972 & 1.8464 & -0.0428 & -2.3 \\
\hline
\end{tabular}

\subsubsection{Variation of Hydrogenation Parameters}


In order to understand further how the hydrogenation conditions would affect the final products, three of the reaction conditions were altered. The pressure, gaseous atmosphere, and solvent-to-coal ratio were all varied in reactions six (6) through twelve (12). The initial hydrogen pressure was lowered from 1000 psig to 500 psig and finally to 100 psig in runs five (5), seven (7), nine (9). Reactions with these same pressures but under an argon atmosphere were performed in runs six (6), eight (8), and ten (10). Finally, the solvent-tocoal ratio was lowered from $3 / 1$ to $2 / 1$ down to $1 / 1$ by

weight in reactions four (4), eleven (11), and twelve (12). All other conditions for these reactions were kept constant and can be seen in Table 2.3.

\subsubsection{Effect of Pressure and Atmosphere}

Reactions five (5) through ten (10) were performed to determine the effect of lowering the initial pressure and employing an inert atmosphere instead of hydrogen on the conversion, product distribution, and pitch characteristics. Each reaction was performed with the $\mathrm{CBB}$ solvent, at a temperature of $400{ }^{\circ} \mathrm{C}$, a solvent-to-coal ratio of $3 / 1$, and run for one hour. The CBB solvent was used for these experiments rather than DACL-L, which showed a higher conversion (both types), because it is a commercially-available material and could be used as is. The Antaeus liquid is not currently available commercially. Each set of reaction conditions was run four times and separated into different softening point pitches by vacuum distillation. Again, a target softening point of $120{ }^{\circ} \mathrm{C}$ was desired though not always achieved.

Figure 2.11 shows the effect of increasing the reaction pressure and changing the atmosphere on the overall conversion, while Figure 2.12 depicts the same effect on the coal-alone conversion of the hydrogenation reactions. As the pressure is increased, regardless of the gaseous atmosphere, both the overall and coal-alone conversions increase. However, the overall conversion actually only increases 3\% from pressures of 100 psig to 1000 psig hydrogen and only $2 \%$ for the same increase in argon pressure. This is an insignificant increase when considering a ten fold increase in pressure was required. The effect of increasing the gas pressure on the overall conversion is masked due to the low amount of coal compared to solvent charged to the reactors. This becomes evident when the effect of gas pressure on the coal-alone conversion is investigated in Figure 2.12. When hydrogen gas is employed the coal-alone conversion increases from $7 \%$ to $23 \%$ when the pressure is raised from 100 psig to 1000 psig. This is a much more significant increase than that shown in Figure 2.11 for the overall conversion. The coal alone conversion also increases ( $6 \%$ to $12 \%)$ when argon pressure is raised from 100 to 1000 psig, however, not as significantly. This shows that when hydrogen gas is being used in the hydrogenation reaction, it is beneficial to the reaction of coal to soluble products. 


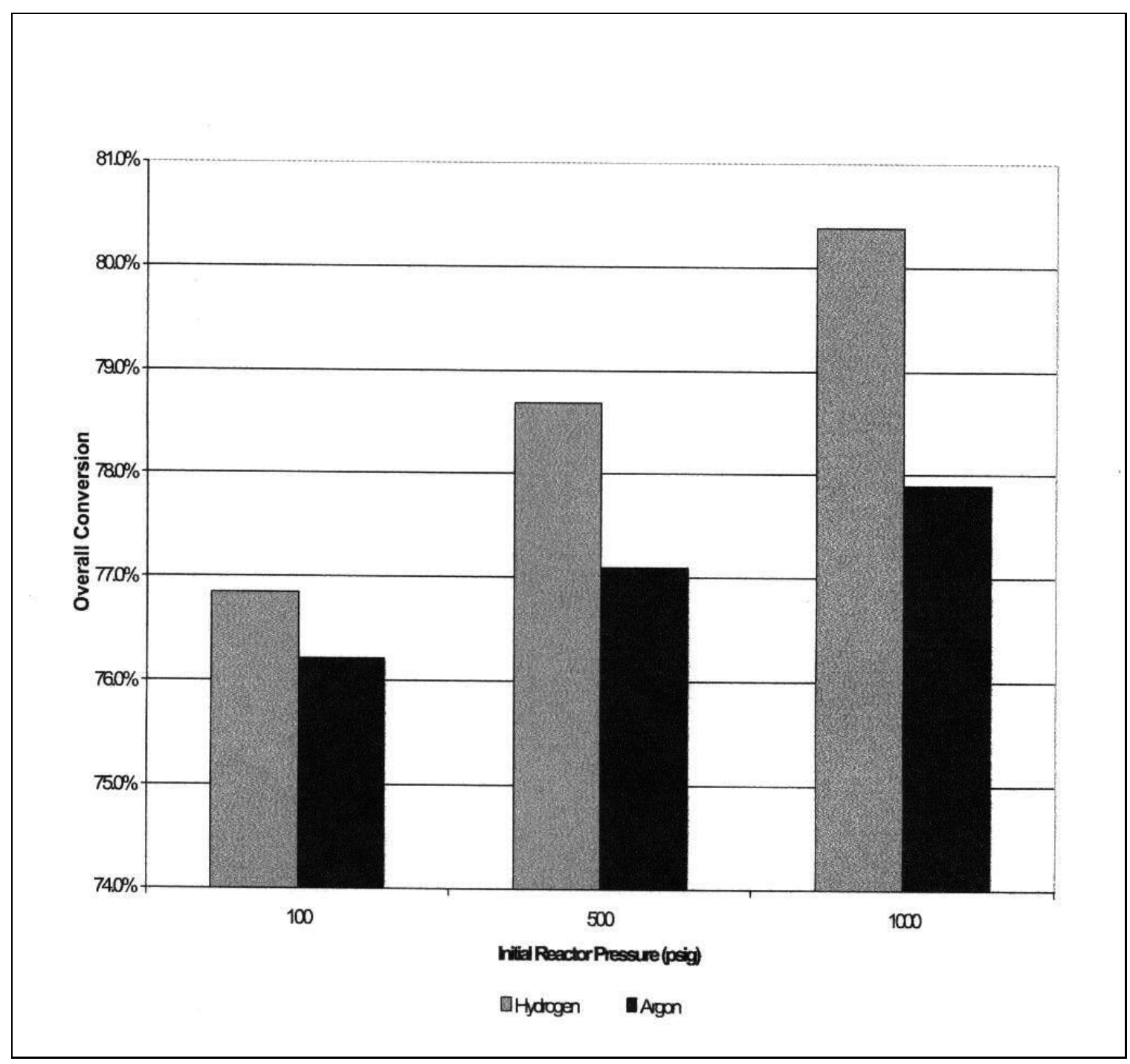

Figure 2.11 Effect of initial reactor pressure and gaseous atmosphere on the overall conversion of hydrogenation reactions with $\mathrm{CBB}$ and reaction conditions of $400{ }^{\circ} \mathrm{C}, 3 / 1$ solvent-to-coal ratio, and 1 hour 


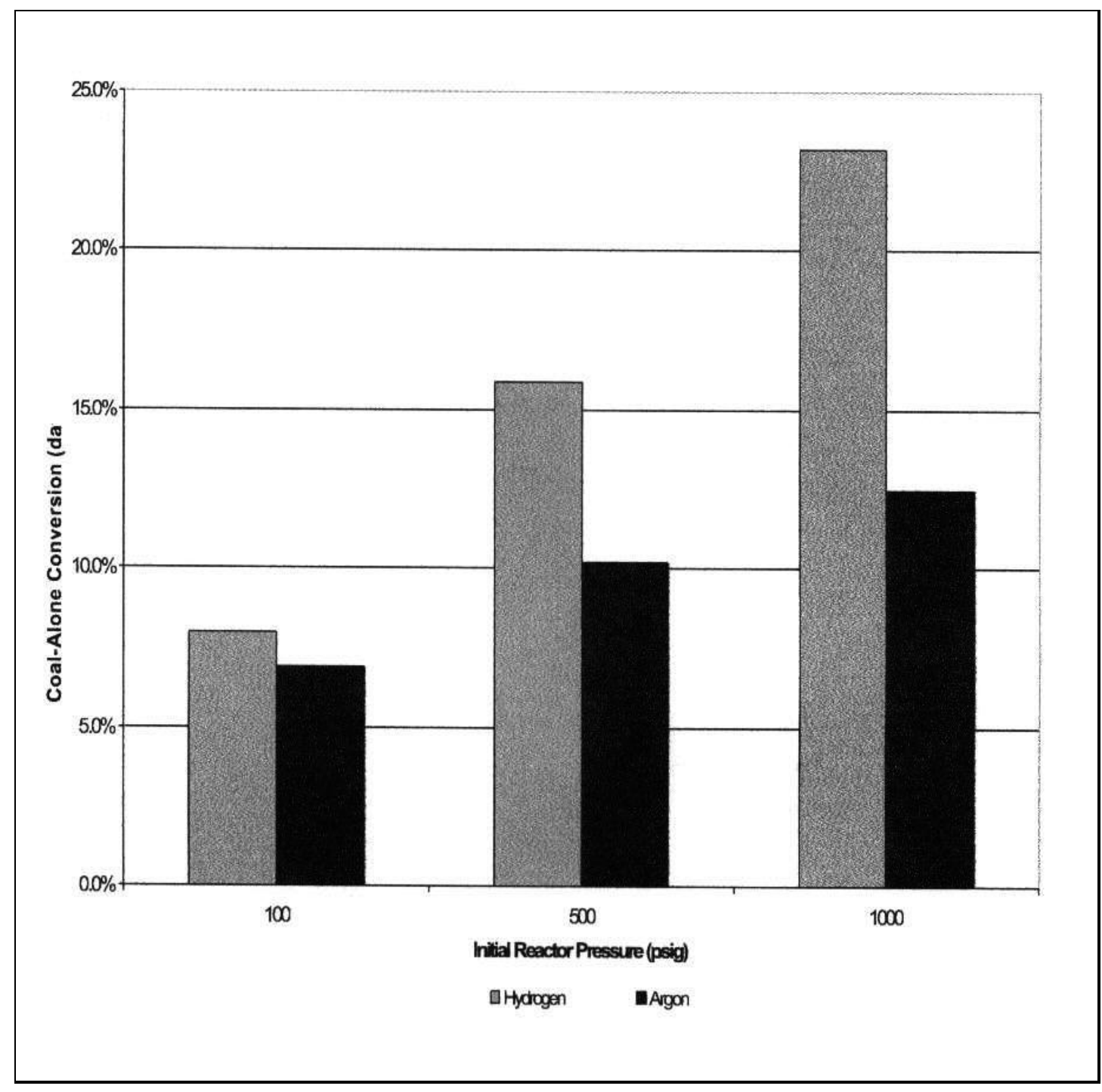

Figure 2.12 Effect of initial reactor pressure and gaseous atmosphere on the coalalone conversion of hydrogenation reactions with $C B B$ and reaction conditions of $400{ }^{\circ} \mathrm{C}, 3 / 1$ solvent-to-coal ratio, and 1 hour

Another way of illustrating the use of gaseous hydrogen in the hydrogenation reactions is to compare the reactor pressures during the thermal treatment when the same initial pressures are employed with hydrogen and argon atmospheres. These results are depicted in Figures $2.13,2.14$, and 2.15 for the initial reaction pressures of 1000,500 , and $100 \mathrm{psig}$, respectively. It is interesting to note that when the initial pressures were either 1000 or 500 psig (Figures 2.13 and 2.14), the hydrogen charged reactors decreased in pressure over time, while the argon charged reactors continued to increase in pressure throughout the reaction time. The decrease in pressure in the hydrogen charged reactions was again due to the fact that the hydrogen was consumed by hydrogenating either transient coal radicals and/or the solvent. 


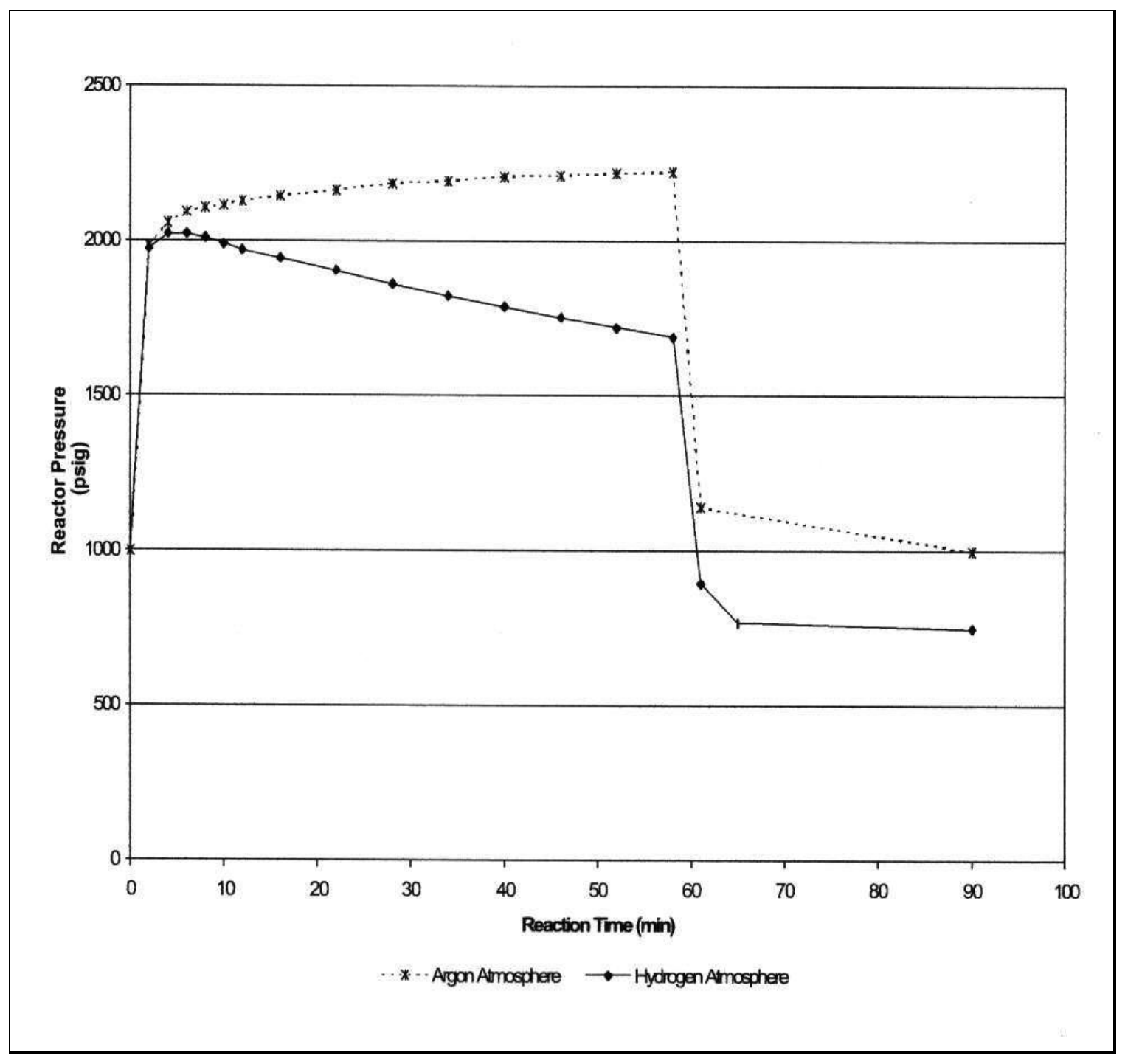

Figure 2.13 Average reactor pressures over time of hydrogenation reactions using $\mathrm{CBB}, 1000$ psig initial hydrogen or argon pressure, $400{ }^{\circ} \mathrm{C}, 3 / 1$ solventto-coal ratio, and 1 hour 


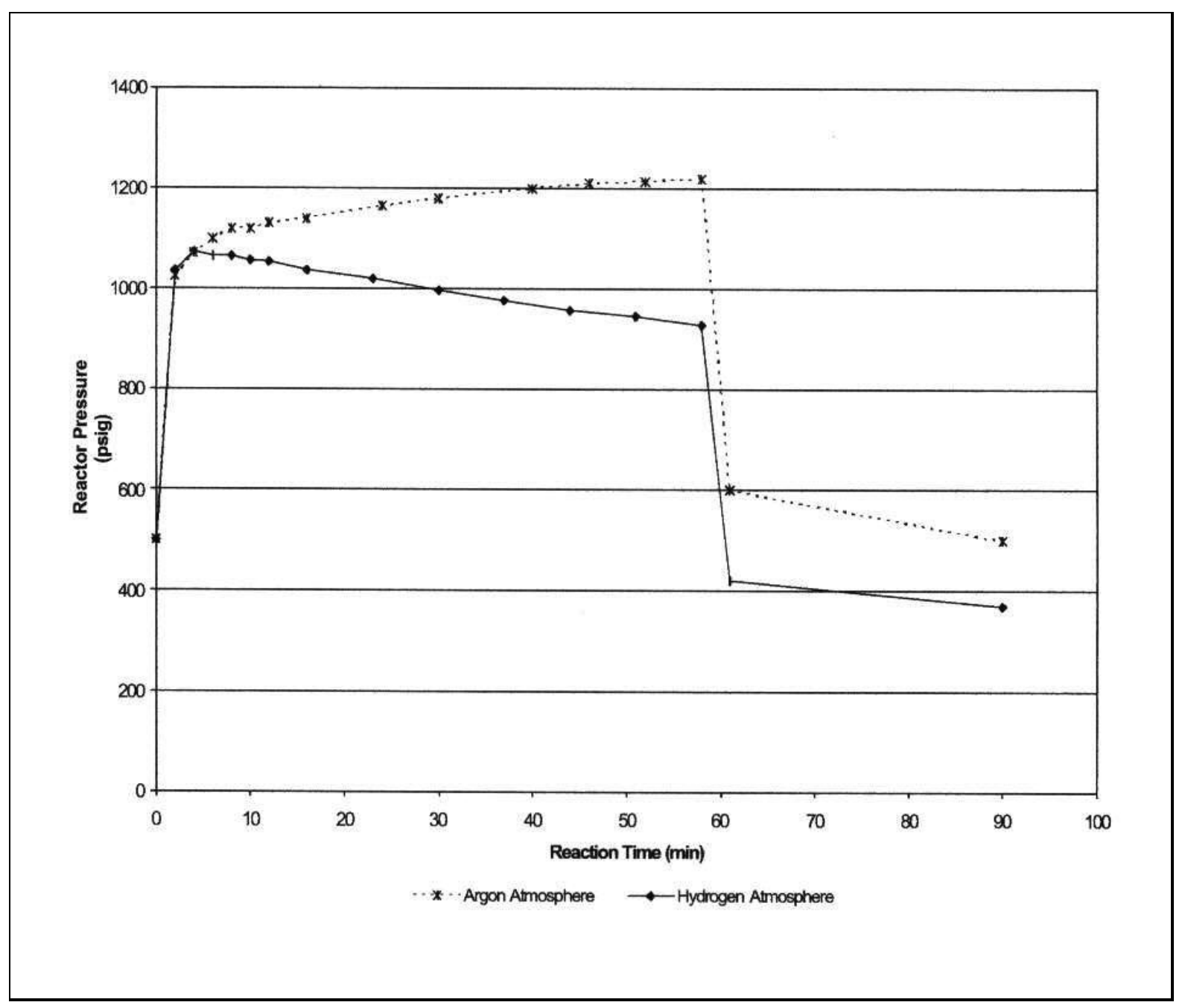

Figure 2.14 Average reactor pressures over time of hydrogenation reactions using $\mathrm{CBB}, 500$ psig initial hydrogen or argon pressure, $400{ }^{\circ} \mathrm{C}, 3 / 1$ solvent-tocoal ratio, and 1 hour 


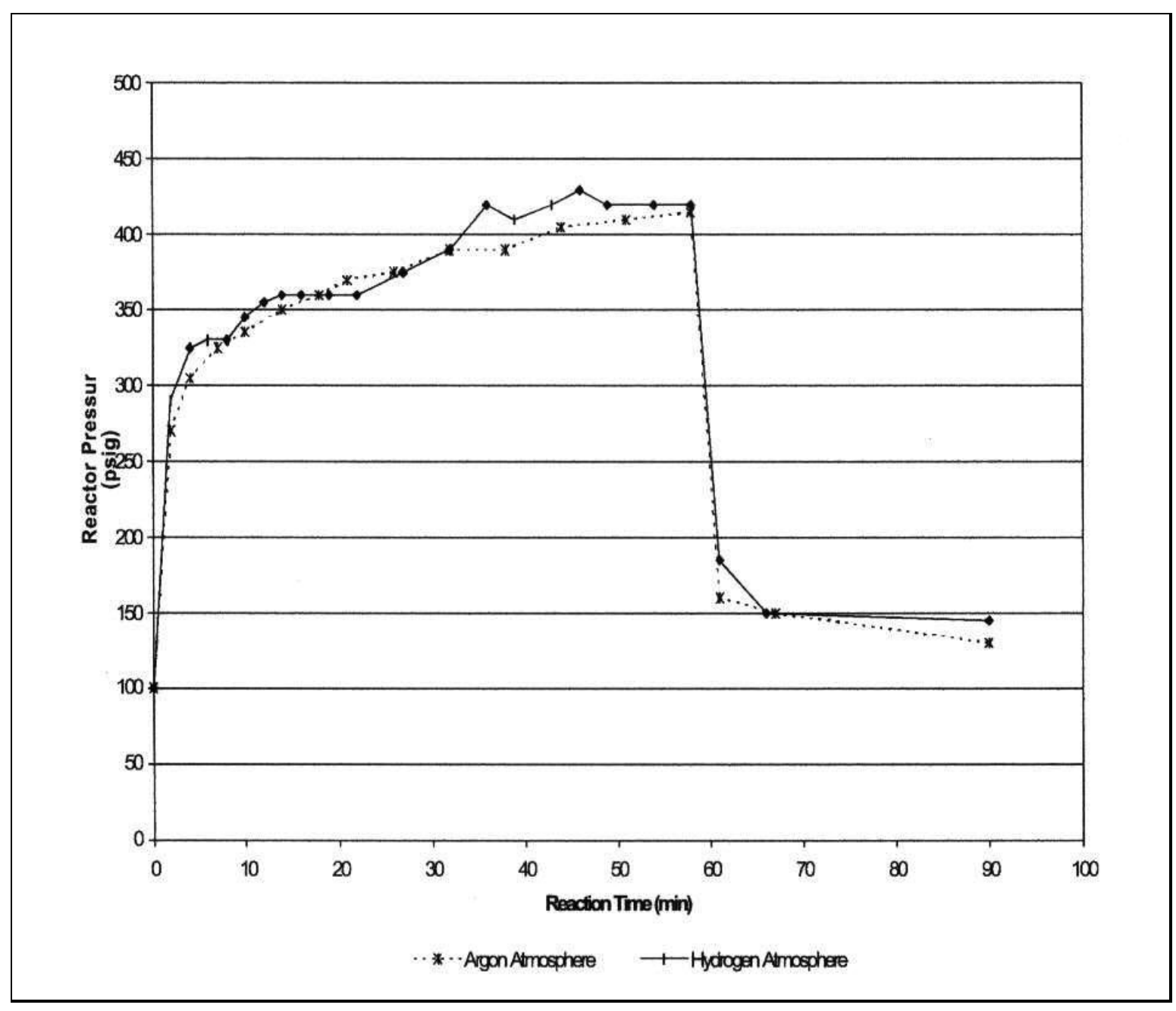

Figure 2.15 Average reactor pressures over time of hydrogenation reactions using $\mathrm{CBB}, 100$ psig initial hydrogen or argon pressure, $400{ }^{\circ} \mathrm{C}, 3 / 1$ solvent-tocoal ratio, and 1 hour

by comparing the initial and final pressures of the reactions performed under a hydrogen atmosphere, an estimation of the amount of hydrogen consumed can be made. The hydrogen consumed for the reactions with 1000 psig initial hydrogen pressure was approximately 0.04 moles of hydrogen or $0.2 \%$ of the reaction feed mass. The hydrogen consumed for the reactions with 500 psig initial hydrogen pressure was approximately 0.02 moles of hydrogen or $0.1 \%$ of the reaction feed mass. The increase in pressure during the argon charged reactions is from the production of gas. However, this gas most likely condensed when the reaction was quenched and cooled, as evidenced by the fact that the final reactor pressure was equal to the initial reactor pressure. This does not mean, however, that no product gases 
were formed during the hydrogen charged reactions -- only that the amount of gas production inside the reactor was less than the amount of hydrogen consumption. This would lead to the net decrease in reactor pressure observed when hydrogen was used in both Figures 2.13 and 2.14 .

Figure 2.15 shows a different trend with the lower initial pressure of $100 \mathrm{psig}$. Both the hydrogen and argon charged reactors increase in pressure over time. Therefore, gas was being produced throughout both sets of reactions. This time in the hydrogen charged reactor, however, the rate of gas production was greater than the rate of hydrogen gas consumption. This explanation is further justified by comparing the initial and final pressures in the reactors. For both systems, the final pressure is greater than the initial pressure, signifying a net production in gas and less hydrogen reacted at 100 psig.

The effect of changing the initial reactor pressure and atmosphere on the product distribution is illustrated in Figure 2.16. The distributions shown represent the products thus formed when a pitch with a softening point in the proximity of $120^{\circ} \mathrm{C}$ was obtained. For both the argon and hydrogen charged reactions, as the pressure is increased the amount of THF insolubles decreased slightly from $23 \%$ to $21 \%$ for argon reactions and 23.5 to $20 \%$ for hydrogen reactions. This is directly responsible for the increase in both the overall and coalalone conversions as more coal is reacted. The percent of pitch and recycle solvent recovered from the feed seems to be fairly constant when experimental error is considered. The percent of pitch obtained with a hydrogen atmosphere increased slightly (from 16\% to 20\%) as the initial reactor pressure increased. This could be attributed to the increase of coal conversion when the hydrogen pressure was increased. The percent of pitch obtained from the feed when using an argon atmosphere does not show such a trend. The percent of feed going to pitch did increase from 100 psig initial argon pressure to 1000 psig initial argon pressure, but a dip was observed when 500 psig initial argon pressure was employed. This dip can be attributed to overshooting the desired softening point of $120^{\circ} \mathrm{C}$. The pitches obtained when using 100 and $1000 \mathrm{psig}$ initial argon pressure had average softening points of 120.3 and $119.3^{\circ} \mathrm{C}$, respectively. The pitch obtained from the reaction charged with $500 \mathrm{psig}$ initial argon pressure was distilled to an average softening point of $132.5^{\circ} \mathrm{C}$ (due to an error in distillation). This increase in softening point corresponds to a smaller amount of pitch produced due to more recycle solvent being removed during distillation. This could explain the dip in the pitch production shown at 500 psig initial argon pressure in Figure 2.16.

After separating the hydrogenation products into the fractions shown in Figure 2.16, the effect of the pressure and atmosphere on the pitch characteristics was studied. The selected pitches with a softening point in the area of $120{ }^{\circ} \mathrm{C}$ (from 119.3 to $132.5^{\circ} \mathrm{C}$ ) were characterized by ash content, coke yield, optical microscopy, and elemental analysis (the corresponding insoluble and recycle solvent fractions were also characterized by elemental analysis). The results of the ash test for the selected pitches and THF insoluble fraction are shown in Table 2.10. As described previously, the ash content of the THF insoluble fraction varies with the conversion of hydrogenation reactants to soluble products. The highest ash content corresponds to the highest conversion (1000 psig Hydrogen initially) due to the decreased amount of organic material left in the THF insoluble fraction. The ash varies 
randomly from pitch to pitch but is always at a very low value (below $0.1 \%$ ). This is important because the ash content is considered an impurity in the development of carbon products.

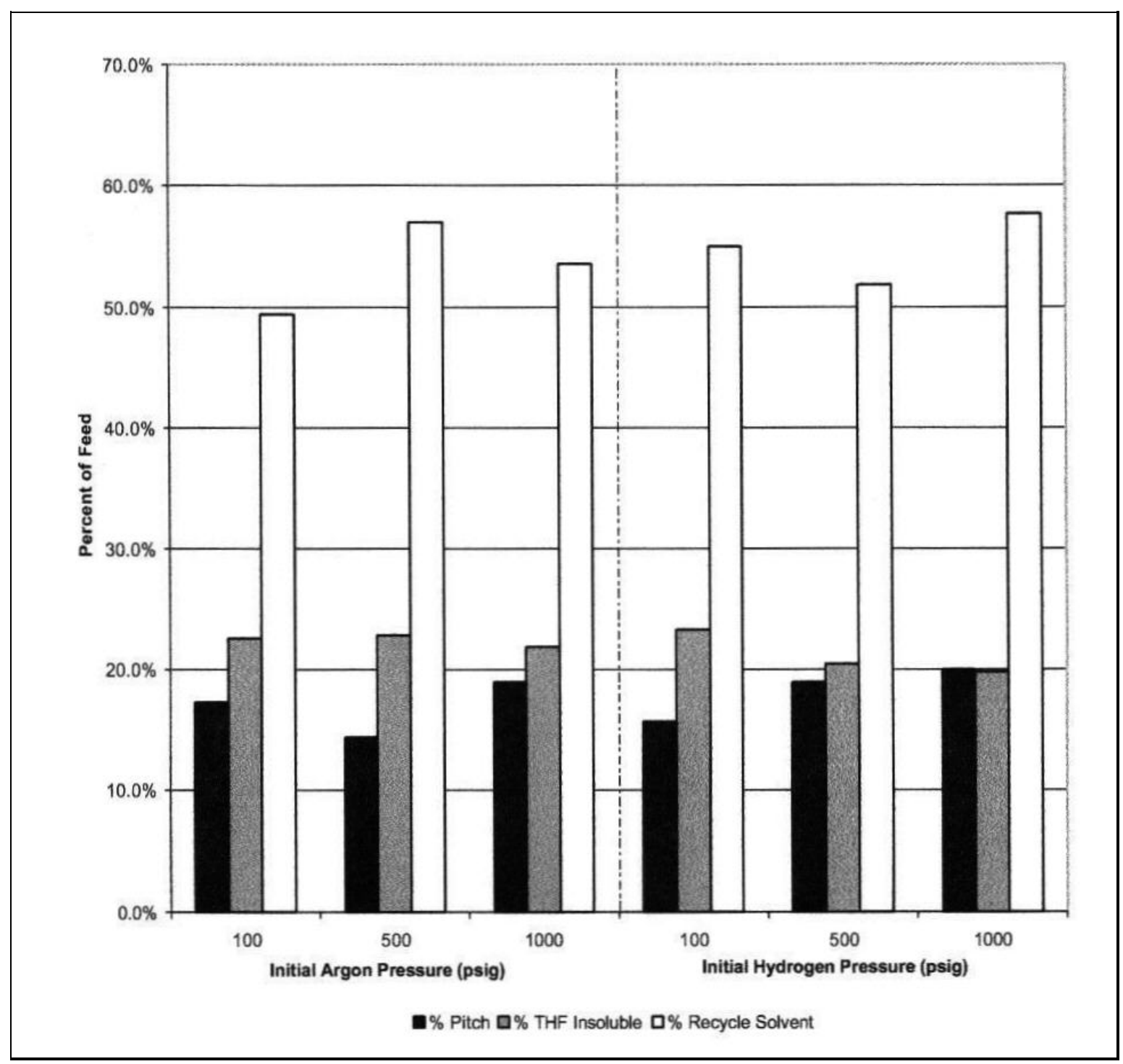

Figure 2.16 Effect of initial reactor pressure and atmosphere on the product distribution of reactions run to produce a pitch with a softening point of $120{ }^{\circ} \mathrm{C}$ under reaction conditions of $\mathrm{CBB}$ solvent, $400{ }^{\circ} \mathrm{C}, 3 / 1$ solventto-coal ratio, and 1 hour

Table 2.10 Ash content of pitch and THF insoluble fractions of pitch produced while 
varying the initial reactor pressure and gaseous atmosphere

\begin{tabular}{|c|c|c|c|c|c|c|c|}
\hline \hline Run & Trial & $\begin{array}{c}\text { Initial } \\
\text { Reactor } \\
\text { Pressure } \\
\text { (psig) }\end{array}$ & Gas & $\begin{array}{c}\text { Pitch } \\
\text { Average } \\
\text { Softening } \\
\text { Point ( }\end{array}$ & $\begin{array}{c}\text { \% } \text { Ash in } \\
\text { Pitch }\end{array}$ & $\begin{array}{c}\% \text { Ash in } \\
\text { THF } \\
\text { Insoluble }\end{array}$ & $\begin{array}{c}\text { Recovered } \\
\text { Ritch }\end{array}$ \\
\hline 5 & B & 1000 & Hydrogen & 126.9 & $0.093 \%$ & $11.10 \%$ & $20.0 \%$ \\
\hline 6 & D & 1000 & Argon & 119.3 & $0.041 \%$ & $7.95 \%$ & $18.9 \%$ \\
\hline 7 & A & 500 & Hydrogen & 113.2 & $0.037 \%$ & $10.15 \%$ & $18.9 \%$ \\
\hline 8 & D & 500 & Argon & 132.5 & $0.017 \%$ & $7.86 \%$ & $14.4 \%$ \\
\hline 9 & B & 100 & Hydrogen & 123.6 & $0.035 \%$ & $8.47 \%$ & $15.6 \%$ \\
\hline 10 & D & 100 & Argon & 120.3 & $0.108 \%$ & $7.91 \%$ & $17.3 \%$ \\
\hline
\end{tabular}

The coke yield of the selected pitches was also determined. However, because the coke yield is very dependent on the softening point of the pitch (as shown earlier by Figure 2.9) and some of the selected pitches (7A and $8 \mathrm{D}$ ) have softening points that deviate greatly from the target of $120^{\circ} \mathrm{C}$, a plot of coke yield versus softening point is used to evaluate the effect of pressure and atmosphere on the pitches' coke yield. This plot is shown in Figure 2.17. The coke yield shows little dependence on the hydrogenation reaction conditions of gas composition and pressure. However, it does depend significantly on the softening point of the pitch. From this plot, it can be determined that a pitch with a softening point of $120^{\circ} \mathrm{C}$ would have a coke yield of approximately $45 \%$ by weight. In order to achieve a coke yield of $50 \%$ by weight, the softening point would have to be increased to approximately $145^{\circ} \mathrm{C}$, and to reach a coke yield of $60 \%$ by weight, a softening point of greater than $180{ }^{\circ} \mathrm{C}$ would be required.

Once the coke samples have been formed, the optical texture can be investigated by optical microscopy. The micrographs of cokes produced from the pitches with a softening point close to $120^{\circ} \mathrm{C}$ (the same ones shown in Table 2.10) are shown in Figure 2.18. Each of these represents a different hydrogenation pressure and atmosphere. Each coke sample has an anisotropic texture with the domain sizes differing depending on the reaction condition used. However, there does not seem to be a direct correlation between the domain sizes in the cokes and the hydrogenation pressure or atmosphere. Therefore, high hydrogen pressures are not required to make anisotropic coke. This is an important result since high pressures and hydrogen incur higher operational costs and hazards. 


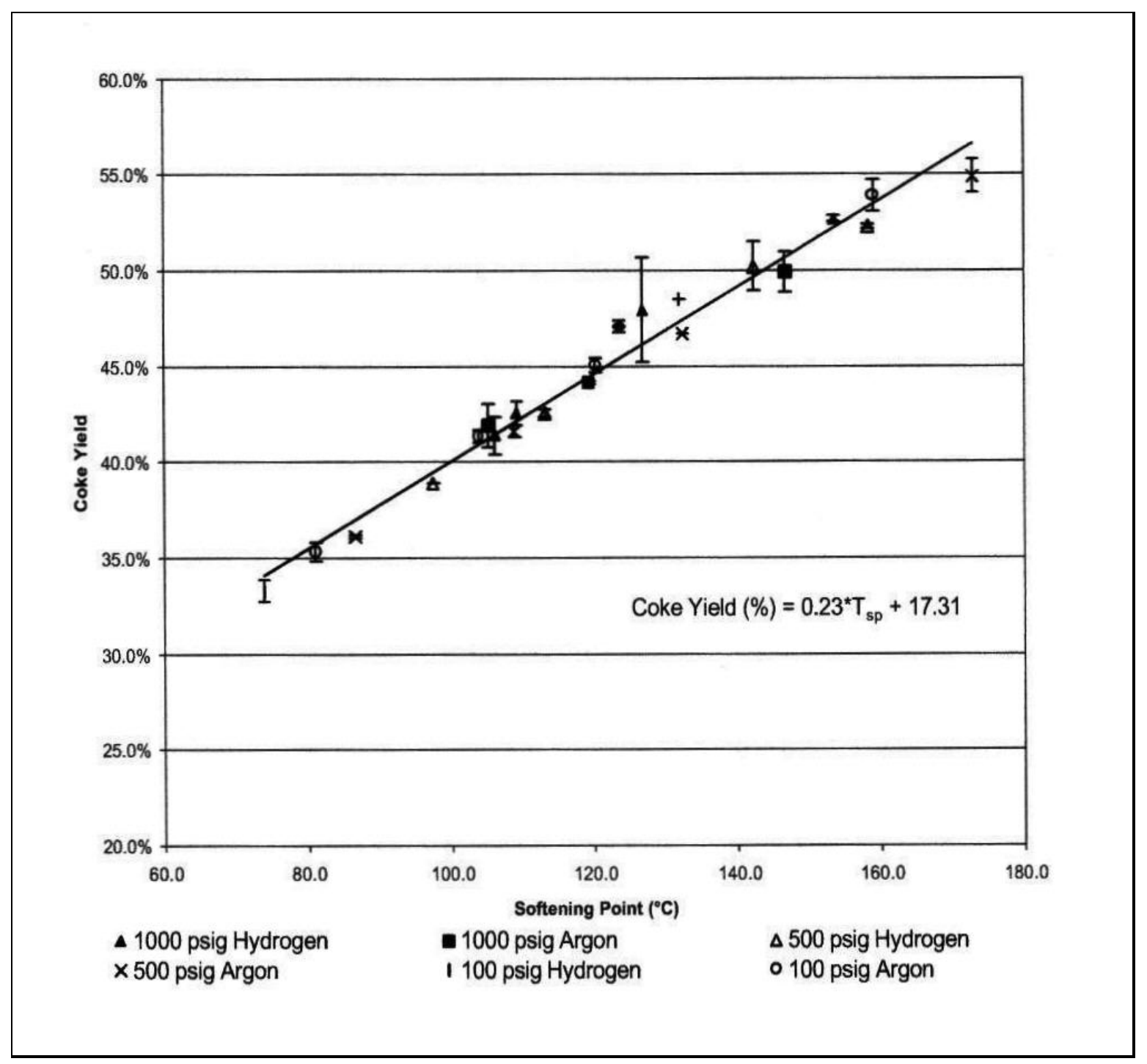

Figure 2.17 Effect of raising the softening point of pitches produced under different atmospheres and initial reaction pressures on the coke yield with reaction conditions of $\mathrm{CBB}$ solvent, $400{ }^{\circ} \mathrm{C}, 3 / 1$ solvent-to-coal ratio, 1 hour 


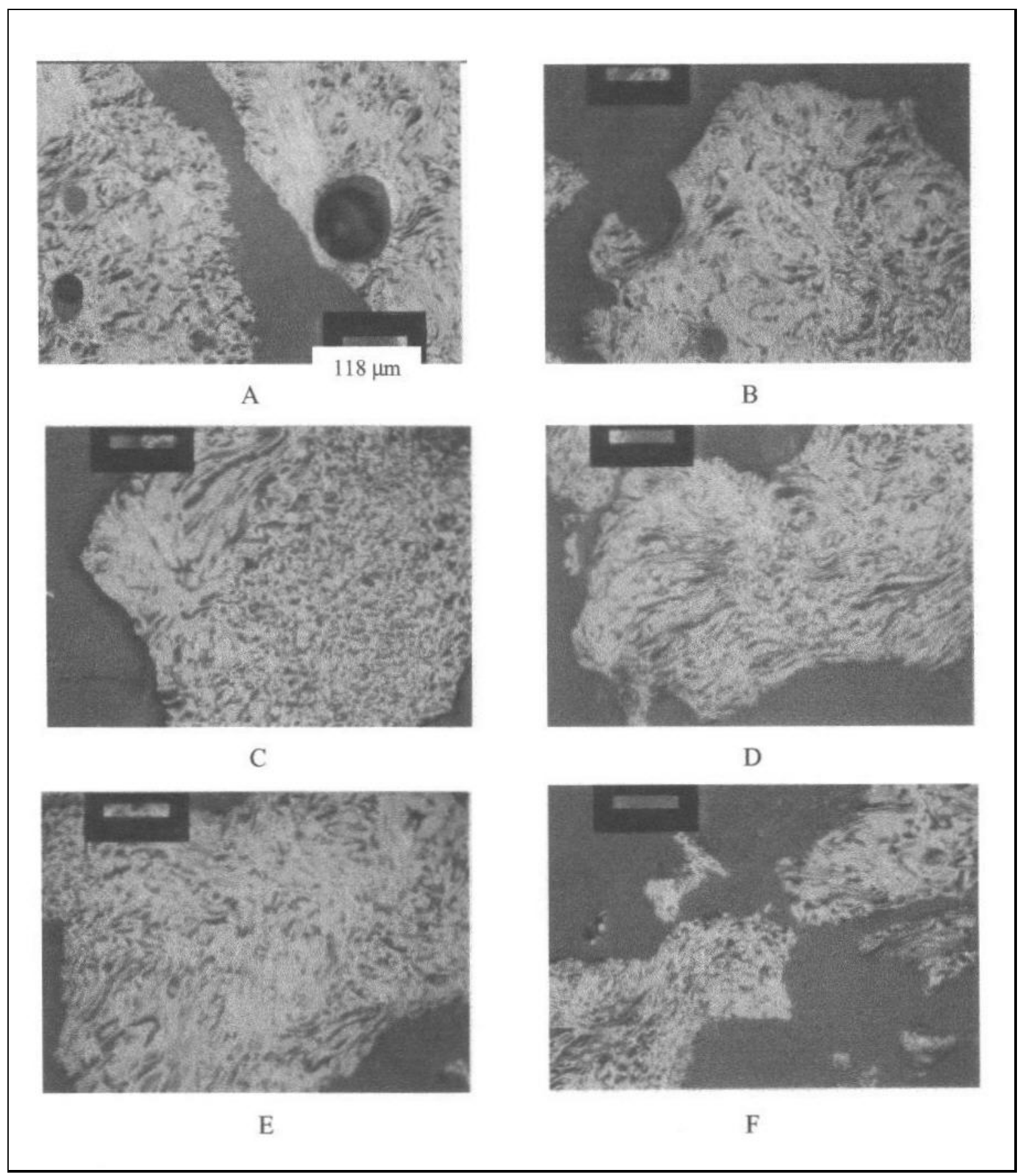

Figure 2.18 Optical micrographs of cokes produced from select pitches derived from hydrogenation products from reactions (CBB solvent, $400{ }^{\circ} \mathrm{C}, 3 / 1$ solvent-to-coal ratio, 1 hour) with varying initial pressures and atmospheres, A) 1000 psig Hydrogen, B) 1000 psig Argon, C) 500 psig Hydrogen, D) 500 psig Argon, E) 100 psig Hydrogen, and F) 100 psig Argon 
The final characterization that was carried out on these hydrogenation products was an elemental analysis. This was done according to the procedure described in Section 2.3.5. The results of the elemental analysis for the three product fractions are shown in Table 2.11 and were also used to calculate a carbon and hydrogen balance (shown in Tables 2.12 and 2.13, respectively). Table 2.11 shows that the elemental composition of each product fraction is approximately equal (within experimental error) for each hydrogenation run, regardless of the initial pressure and atmosphere. This could be due to the low conversions achieved which lead to a significant amount of original solvent left in the pitch fraction. Table 2.12 and Table 2.13 reveal a negative carbon and hydrogen balance for the selected hydrogenation reactions. This deviation can be attributed to depending on the mass balance to calculate the carbon and hydrogen balances. The reason for these errors is similar to that described for the previous carbon and hydrogen balances in Section 2.4.6.4. However, it is important to note that the hydrogen balance is more accurate for the runs with added hydrogen pressure than those with argon. This indicates that more hydrogen was being added when hydrogen was employed as the gas phase.

\subsubsection{Effect of Solvent-to-Coal Ratio}

Thus far, the carbon product precursors (pitches) have had a very desirable ash content and optical texture, but the coke yield for the pitches with a softening point in an softening point in order to achieve a higher coke yield has been thoroughly discussed in Section 2.4.6.2 and the middle of Section 2.4.7.1. However, there could be another alternative to raising the softening point to increase the coke yield. By lowering the solvent-to-coal ratio, less solvent would be retained by the produced pitch. Since the retained solvent would generally be volatilized during the coking process, decreasing the amount of solvent retained by the pitch could increase the coke yield by increasing the proportion of heavier coal-derived species, again assuming lowering the solvent-to-coal ratio does not affect the coal conversion. Hydrogenation runs four (4), eleven (11) and twelve (12) explore this option. For the three sets of reactions, $\mathrm{HCO}$ was employed as the solvent (again because it is more commercially available than DACL-L, however because of limited quantities of $\mathrm{HCO}$, it was only used for this test and not in the previous section), and the reactions were carried out at $400{ }^{\circ} \mathrm{C}, 1000$ psig initial hydrogen pressure, 1 hour, and a decreasing solvent-to-coal ratio (by weight) of $3 / 1$ for run four (4), 2/1 for run eleven (11), and 1/1 for run twelve (12). The effect of lowering the solvent-to-coal ratio on the overall and coal-alone conversions, ash content, coking value, and elemental analysis is discussed below.

Table 2.11 Elemental composition of select pitches produced from hydrogenation reactions (CBB solvent, $400{ }^{\circ} \mathrm{C}, 3 / 1$ solvent-to-coal ratio, 1 hour) with different initial pressures and atmospheres: 5B) 1000 psig Hydrogen, 
6D)

1000 psig Argon, 7A) 500 psig Hydrogen, 8D) 500 psig Argon, 9B) 100 psig Hydrogen, and 10D) 100 psig Argon

\begin{tabular}{|c|c|c|c|c|c|}
\hline \multirow{2}{*}{\begin{tabular}{|c|} 
Run \\
(Initial \\
Pressure \\
psig) \\
\end{tabular}} & \multirow[b]{2}{*}{ Element } & \multirow[b]{2}{*}{$\begin{array}{c}\text { Original } \\
\text { Solvent CBB }\end{array}$} & \multicolumn{3}{|c|}{ Product Fraction } \\
\hline & & & Pitch & $\begin{array}{c}\text { THF } \\
\text { Insoluble }\end{array}$ & $\begin{array}{l}\text { Recycle } \\
\text { Solvent }\end{array}$ \\
\hline \multirow{5}{*}{$\begin{array}{c}5 \mathrm{~B} \\
\left(1000 \mathrm{H}_{2}\right)\end{array}$} & C & $91.85 \%$ & $89.75 \%$ & $79.17 \%$ & $90.73 \%$ \\
\hline & $\mathrm{H}$ & $5.14 \%$ & $5.14 \%$ & $3.97 \%$ & $5.79 \%$ \\
\hline & $\mathrm{N}$ & $0.88 \%$ & $1.15 \%$ & $0.86 \%$ & $0.89 \%$ \\
\hline & $\mathrm{S}$ & $0.55 \%$ & $0.63 \%$ & $0.94 \%$ & $0.69 \%$ \\
\hline & $\begin{array}{l}\text { H/C Atomic } \\
\text { Ratio }\end{array}$ & 0.66 & 0.69 & 0.60 & 0.77 \\
\hline \multirow{5}{*}{$\begin{array}{c}6 \mathrm{D} \\
(1000 \mathrm{Ar})\end{array}$} & $\mathrm{C}$ & $91.85 \%$ & $91.42 \%$ & $82.60 \%$ & $92.89 \%$ \\
\hline & $\mathrm{H}$ & $5.14 \%$ & $5.31 \%$ & $3.91 \%$ & $5.51 \%$ \\
\hline & $\mathrm{N}$ & $0.88 \%$ & $1.29 \%$ & $1.08 \%$ & $0.45 \%$ \\
\hline & $S$ & $0.55 \%$ & $0.84 \%$ & $0.78 \%$ & $0.78 \%$ \\
\hline & $\begin{array}{c}\text { H/C Atomic } \\
\text { Ratio }\end{array}$ & 0.66 & 0.70 & 0.57 & 0.71 \\
\hline \multirow{5}{*}{$\begin{array}{c}7 \mathrm{~A} \\
\left(500 \mathrm{H}_{2}\right)\end{array}$} & $\mathrm{C}$ & $91.85 \%$ & $91.51 \%$ & $82.49 \%$ & $91.26 \%$ \\
\hline & $\mathrm{H}$ & $5.14 \%$ & $5.27 \%$ & $4.09 \%$ & $5.75 \%$ \\
\hline & $\mathrm{N}$ & $0.88 \%$ & $1.68 \%$ & $1.24 \%$ & $0.01 \%$ \\
\hline & $\mathrm{S}$ & $0.55 \%$ & $0.77 \%$ & $0.88 \%$ & $0.63 \%$ \\
\hline & $\begin{array}{c}\text { H/C Atomic } \\
\text { Ratio }\end{array}$ & 0.66 & 0.69 & 0.59 & 0.76 \\
\hline \multirow{5}{*}{$\begin{array}{c}8 \mathrm{D} \\
(500 \mathrm{Ar})\end{array}$} & C & $91.85 \%$ & $92.50 \%$ & $81.41 \%$ & $89.59 \%$ \\
\hline & $\mathrm{H}$ & $5.14 \%$ & $5.04 \%$ & $4.21 \%$ & $5.27 \%$ \\
\hline & $\mathrm{N}$ & $0.88 \%$ & $1.73 \%$ & $1.92 \%$ & $0.59 \%$ \\
\hline & $\mathrm{s}$ & $0.55 \%$ & $0.79 \%$ & $0.81 \%$ & $0.76 \%$ \\
\hline & $\begin{array}{l}\mathrm{H} / \mathrm{C} \text { Atomic } \\
\text { Ratio }\end{array}$ & 0.66 & 0.65 & 0.62 & 0.71 \\
\hline \multirow{5}{*}{$\begin{array}{c}9 \mathrm{~B} \\
\left(100 \mathrm{H}_{2}\right)\end{array}$} & $\mathrm{C}$ & $91.85 \%$ & $91.55 \%$ & $82.22 \%$ & $92.09 \%$ \\
\hline & $\mathrm{H}$ & $5.14 \%$ & $5.19 \%$ & $4.08 \%$ & $5.80 \%$ \\
\hline & $\mathrm{N}$ & $0.88 \%$ & $1.12 \%$ & $1.30 \%$ & $0.87 \%$ \\
\hline & $\mathrm{S}$ & $0.55 \%$ & $0.69 \%$ & $0.84 \%$ & $0.61 \%$ \\
\hline & $\begin{array}{l}\text { H/C Atomic } \\
\text { Ratio }\end{array}$ & 0.66 & 0.68 & 0.60 & 0.76 \\
\hline \multirow{5}{*}{$\begin{array}{c}10 \mathrm{D} \\
(100 \mathrm{Ar})\end{array}$} & $\mathrm{C}$ & $91.85 \%$ & $89.04 \%$ & $82.02 \%$ & $89.58 \%$ \\
\hline & $\mathrm{H}$ & $5.14 \%$ & $5.07 \%$ & $4.19 \%$ & $5.47 \%$ \\
\hline & $\mathrm{N}$ & $0.88 \%$ & $1.51 \%$ & $1.20 \%$ & $0.77 \%$ \\
\hline & $\mathrm{S}$ & $0.55 \%$ & $0.80 \%$ & $0.64 \%$ & $0.46 \%$ \\
\hline & $\begin{array}{l}\text { H/C Atomic } \\
\text { Ratio }\end{array}$ & 0.66 & 0.68 & 0.61 & 0.73 \\
\hline
\end{tabular}


Table 2.12 Carbon balance of select pitches produced from hydrogenation reactions with different initial pressures and atmospheres: 5B) 1000 psig Hydrogen, 6D) 1000 psig Argon, 7A) 500 psig Hydrogen, 8D) 500 psig Argon, 9B) 100 psig Hydrogen, and 10D) 100 psig Argon

\begin{tabular}{|c|c|c|c|c|c|c|c|c|c|c|}
\hline \hline & & $\begin{array}{c}\text { Carbon } \\
\text { In Coal } \\
\text { Run }\end{array}$ & $\begin{array}{c}\text { Carbon } \\
\text { In Solv. } \\
\text { (g) }\end{array}$ & $\begin{array}{c}\text { Total } \\
\text { Carbon In } \\
\text { (g) }\end{array}$ & $\begin{array}{c}\text { Carbon } \\
\text { Out Pitch } \\
(\mathrm{g})\end{array}$ & $\begin{array}{c}\text { Carbon } \\
\text { Out THF } \\
\text { Ins. (g) }\end{array}$ & $\begin{array}{c}\text { Carbon Out } \\
\text { Rec. Solv. } \\
(\mathrm{g})\end{array}$ & $\begin{array}{c}\text { Total } \\
\text { Carbon } \\
\text { Out (g) }\end{array}$ & $\begin{array}{c}\text { Out - In } \\
(\mathrm{g})\end{array}$ & $\begin{array}{c}\text { (Out-In)/In } \\
(\%)\end{array}$ \\
\hline 5 & B & 7.3079 & 24.6799 & 31.9878 & 6.4201 & 5.6164 & 18.7533 & 30.7898 & -1.1980 & -3.7 \\
\hline 6 & D & 7.2931 & 24.6307 & 31.9238 & 5.9574 & 6.4492 & 17.8073 & 30.2139 & -1.7099 & -5.4 \\
\hline 7 & A & 6.9826 & 23.5819 & 30.5646 & 5.9244 & 5.7824 & 16.1809 & 27.8877 & -2.6768 & -8.8 \\
\hline 8 & D & 7.0193 & 23.7043 & 30.7236 & 4.5702 & 6.3890 & 17.5604 & 28.5195 & -2.2041 & -7.2 \\
\hline 9 & B & 7.0901 & 23.9469 & 31.0369 & 4.9749 & 6.6739 & 17.5893 & 29.2381 & -1.7989 & -5.8 \\
\hline 10 & D & 7.2111 & 24.3504 & 31.5614 & 5.4455 & 6.5388 & 15.6412 & 27.6255 & -3.9359 & -12.5 \\
\hline
\end{tabular}

Table 2.13 Hydrogen balance of select pitches produced from hydrogenation reactions with different initial pressures and atmospheres: 5B) 1000 psig Hydrogen, 6D) 1000 psig Argon, 7A) 500 psig Hydrogen, 8D) 500 psig Argon, 9B) 100 psig Hydrogen, and 10D) 100 psig Argon

\begin{tabular}{|c|c|c|c|c|c|c|c|c|c|c|}
\hline Run & Trial & $\begin{array}{c}\text { Hydrogen } \\
\text { in Coal }(\mathrm{g})\end{array}$ & $\begin{array}{c}\text { Hydrogen } \\
\text { in Solv. } \\
\text { (g) }\end{array}$ & $\begin{array}{c}\text { Total } \\
\text { Hydrogen } \\
\text { In (g) } \\
\end{array}$ & $\begin{array}{c}\text { Hydrogen } \\
\text { Out Pitch } \\
\text { (g) }\end{array}$ & $\begin{array}{l}\text { Hydrogen } \\
\text { Out THF } \\
\text { Ins. (g) } \\
\end{array}$ & $\begin{array}{l}\text { Hydrogen } \\
\text { Out Rec. } \\
\text { Solv. (g) }\end{array}$ & $\begin{array}{c}\text { Total } \\
\text { Hydrogen } \\
\text { Out }(\mathrm{g}) \\
\end{array}$ & $\begin{array}{l}\text { Out - In } \\
\text { (g) }\end{array}$ & $\begin{array}{c}\text { (Out-In) In } \\
(\%)\end{array}$ \\
\hline 5 & B & 0.4088 & 1.4804 & 1.8892 & 0.3676 & 0.2817 & 1.1972 & 1.8464 & -0.0428 & -2.3 \\
\hline 6 & D & 0.4080 & 1.4775 & 1.8854 & 0.3461 & 0.3056 & 1.0562 & 1.7080 & -0.1775 & -9.4 \\
\hline 7 & A & 0.3906 & 1.4146 & 1.8052 & 0.3415 & 0.2866 & 1.0201 & 1.6482 & -0.1570 & -8.7 \\
\hline 8 & D & 0.3927 & 1.4219 & 1.8146 & 0.2691 & 0.3302 & 1.0323 & 1.6317 & -0.1829 & -10.1 \\
\hline 9 & B & 0.3966 & 1.4364 & 1.8331 & 0.2822 & 0.3310 & 1.1074 & 1.7206 & -0.1124 & -6.1 \\
\hline 10 & D & 0.4034 & 1.4607 & 1.8640 & 0.3099 & 0.3341 & 0.9551 & 1.5991 & -0.2650 & -14.2 \\
\hline
\end{tabular}

The overall and coal-alone conversions for runs four (4), eleven (11), and twelve (12) are 
depicted in Figure 2.19. It is interesting to note that the overall conversion decreases as the solvent-to-coal ratio decreases, but the coal-alone conversion remains relatively constant (within experimental error). This shows that the coal-alone conversion does not depend significantly on the amount of solvent, only on the reaction conditions. The loss in overall conversion depicted in Figure 2.19 can be attributed to less solvent being carried into the THF soluble fraction. This is expected since there is less solvent in the original reaction feed as the solvent-to-coal ratio is decreased. The result of the coal-alone conversion section of Figure 2.19 is that the coal conversion is independent of the solvent-to-coal ratio. Therefore, the least amount of solvent that allows adequate processing of the reactants and products could be employed. From a coal-alone conversion standpoint, the optimum solvent-to-coal ratio is $2 / 1$ because the $1 / 1$ ratio would not flow when heated, leading to significant processing problems.

Once the THF extraction was completed, the THF soluble fraction was separated by vacuum distillation into the pitch and recycle solvent fractions. This was done in such a way that pitches of several different softening points were obtained, including a sample from each run close to the target value of $120^{\circ} \mathrm{C}$. Figure 2.20 shows a nearly linear dependence of the final softening point of the pitch with the percentage of pitch recovered from the original reaction feed. It is interesting to note that as the solvent-to-coal ratio is decreased, the linear representation of softening point versus percent pitch yield shifts to the right in Figure 2.20. This shift corresponds to less distillation required to achieve a similar softening point as the solvent-to-coal ratio is decreased. This phenomenon is expected since a lower solvent content in the feed suggests that less recycle solvent will be recovered and less solvent needs to be distilled from the pitch to achieve the same softening point. This has some advantages and disadvantages. The advantages are that it produces a higher pitch yield and good coal conversion, but the disadvantages are that the products will be harder to process and less recycle solvent will be generated.

After the distillation was performed, three pitches were isolated (one from each run) with a softening point at or near the $120^{\circ} \mathrm{C}$. These runs were $4 \mathrm{C}, 11 \mathrm{~B}$ and $12 \mathrm{~A}$ and had a softening point of $119.4,127.5$ and $124.6^{\circ} \mathrm{C}$, respectively. These three samples were used to evaluate the ash and elemental content of the resultant products.

The ash content of the pitch and THF insoluble fraction of these three trials are shown in Table 2.14. For each of the selected samples, the ash content of the pitch and insoluble fractions are very similar. This is consistent with the fact that the coal-alone conversion remains constant as the solvent-to-coal ratio is lowered. 


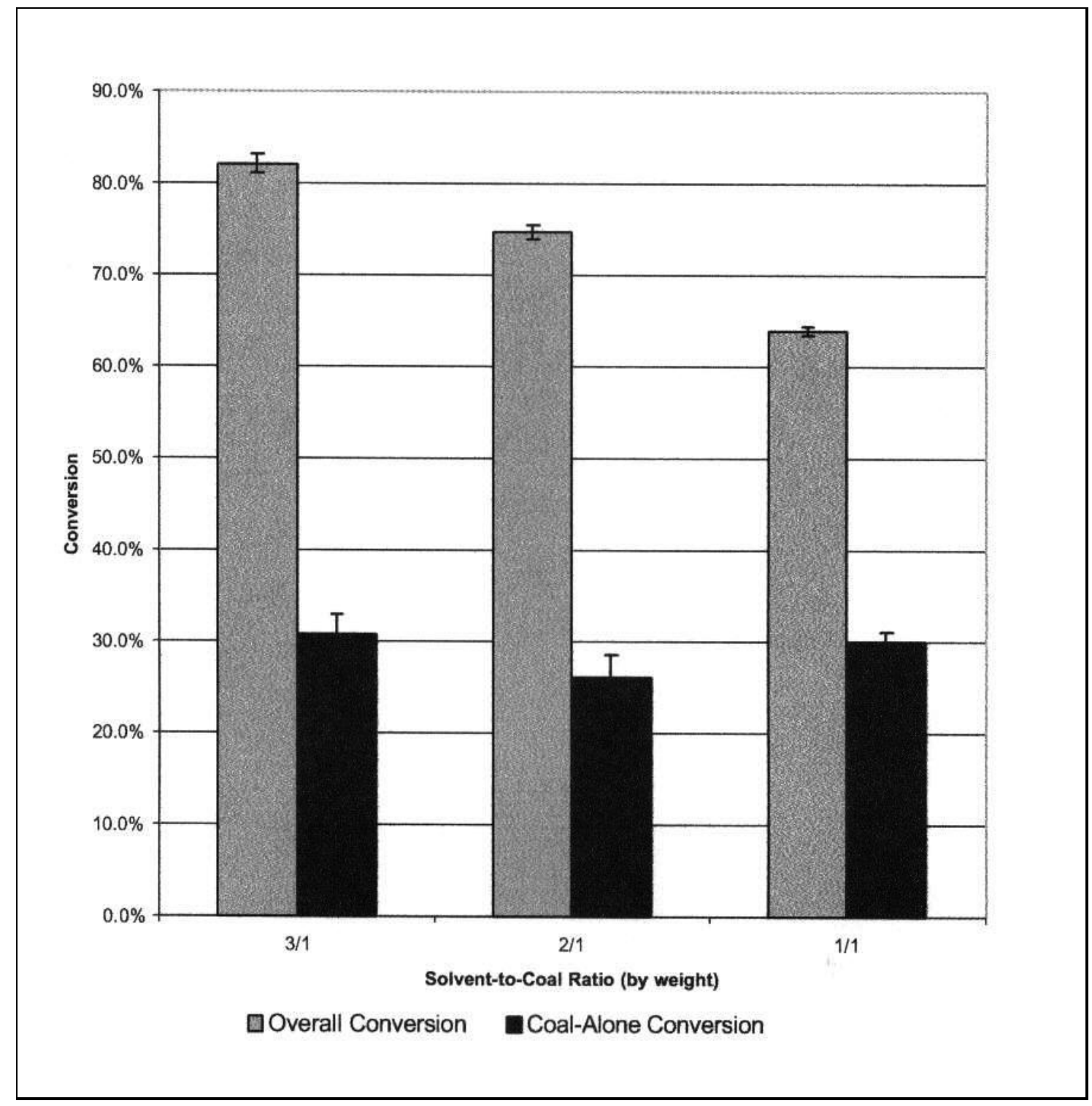

Figure 2.19 Effect of solvent-to-coal ratio on the overall and coal-alone conversions of hydrogenation reactions performed with the HCO solvent under conditions of $400{ }^{\circ} \mathrm{C}, 1000$ psig Hydrogen, and 1 hour 


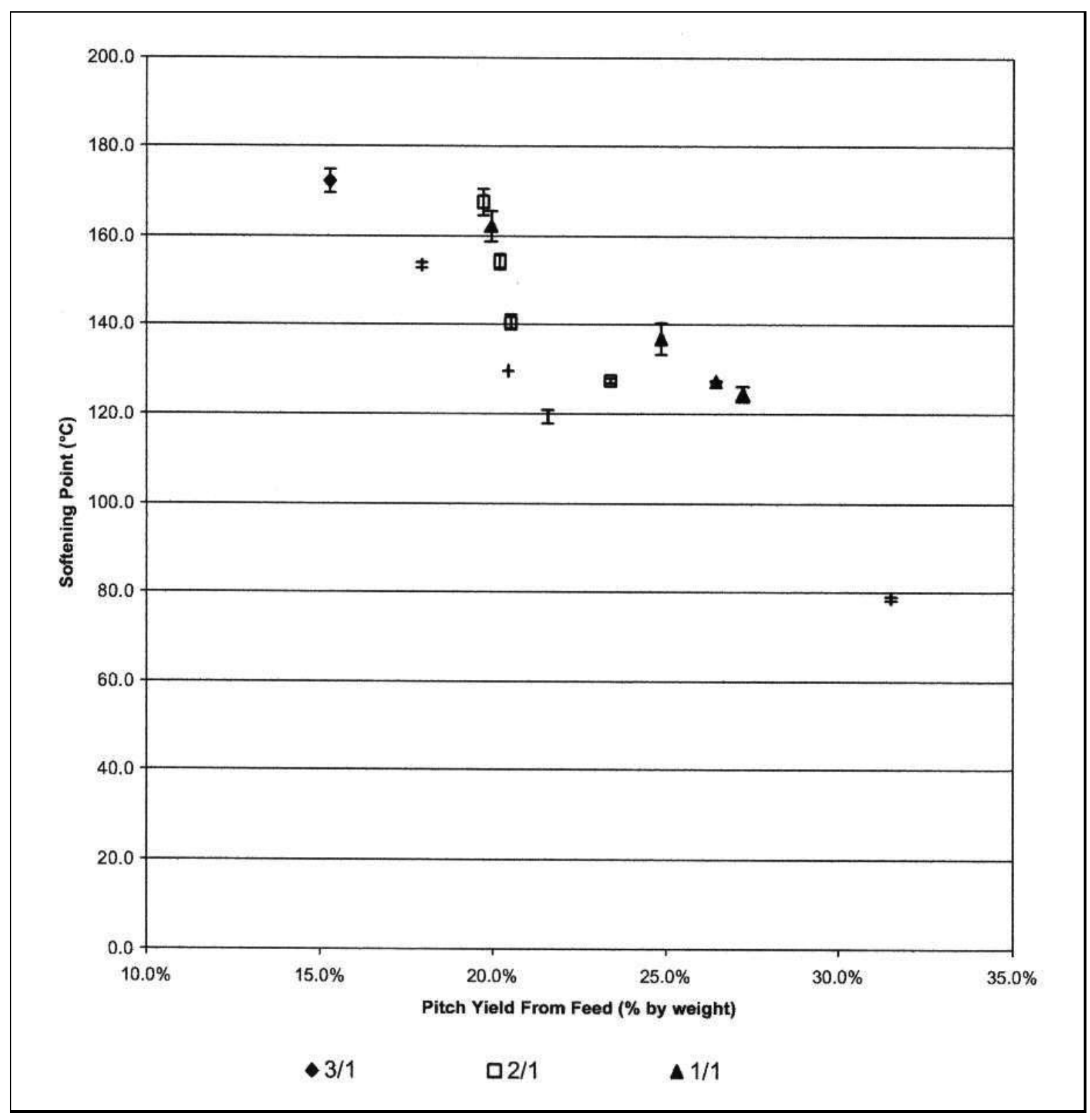

Figure 2.20 Effect of solvent-to-coal ratio on the amount of distillation required to achieve a specific softening point from hydrogenation reactions with conditions of $\mathrm{HCO}$ solvent, $400{ }^{\circ} \mathrm{C}, 1000$ psig hydrogen, and 1 hour 
As with the previous experiments, an elemental analysis was conducted on the hydrogenation products for the selected trials as the solvent-to-coal ratio was lowered and the results of these analyses are shown in Table 2.15. These results indicate that in general the carbon content of each of the pitch fractions decreases (from 91.16\% to 87.29\%) as the solvent-to-coal ratio decreases. However, the hydrogen content remains constant at approximately $5.3 \%$. The carbon and hydrogen contents of the THF insoluble and recycle solvent fractions are all within experimental error of each other, thus showing no dependence on the solvent-to-coal ratio of the hydrogenation reactions. The elemental compositions of the pitch fractions in Table 2.15 are also similar to those of the pitch fractions shown in Tables 2.7 and 2.11. Each pitch fraction has a carbon content of approximately $90 \%$ and a hydrogen content between 5 and $6 \%$ regardless of reaction conditions or the solvent employed.

Table 2.14 Ash content of pitch and THF insoluble fractions of selected hydrogenation runs with decreasing solvent-to-coal ratio with reaction conditions of $\mathrm{HCO}$ solvent, $400{ }^{\circ} \mathrm{C}, 1000$ psig hydrogen, and 1 hour

\begin{tabular}{|l|l|l|l|l|l||}
\hline \multirow{2}{*}{ Run } & Trial & $\begin{array}{l}\text { Solvent-to- } \\
\text { Coal Ratio }\end{array}$ & $\begin{array}{l}\text { Average } \\
\text { Point }\left({ }^{\circ} \mathrm{C}\right)\end{array}$ & $\begin{array}{l}\% \text { Ash in } \\
\text { Softening Ash inTHF } \\
\text { Insoluble } \\
\text { Pitch }\end{array}$ \\
\hline 4 & C & $3 / 1$ & 119.4 & $0.019 \%$ & $10.63 \%$ \\
\hline 11 & B & $2 / 1$ & 127.5 & $0.084 \%$ & $10.80 \%$ \\
\hline 12 & A & $1 / 1$ & 124.6 & $0.024 \%$ & $10.15 \%$ \\
\hline
\end{tabular}

Finally, the effect of lowering the solvent-to-coal ratio on the coke yield of the resultant pitch was investigated. It was hypothesized that by lowering the solvent-to-coal ratio, the amount of solvent remaining in the pitch fraction after distillation would decrease. This decrease in solvent content would cause an increase in the coke yield. Again, because of the dependence of the coke yield on the softening point, a plot of coke yield versus softening point was produced as Figure 2.21 in order to illustrate this effect. However, as Figure 2.21 illustrates, lowering the solvent-to-coal ratio has little or no effect on the coke yield thus proving the previous hypothesis invalid. All three solvent-to-coal ratios seem to fall along a similar linear representation of coke yield versus softening point. It is interesting to note that in order to achieve a coke yield of 50 percent by weight, according to Figure 2.21, a softening point of approximately $145^{\circ} \mathrm{C}$ would be required. 
This linear representation is also similar to that shown in both Figure 2.9 and Figure 2.17. The equations of the linear representations for each graph are very similar. The slopes of the lines for Figures 2.9, 2.17, and 2.21 are $0.21,0.23$, and 0.24 , respectively. The y-intercept for each figure is 19, 17, and 15 for Figures 2.9, 2.17, and 2.21. This similarity in equations leads to producing a plot (Figure 2.22) of coke yield versus softening point for all pitches produced. Figure 2.22 shows that the linear trends from the three previous figures $(2.9,2.17$, and 2.21) are virtually all on the same line. This illustrates that once the pitch is formed, the relationship between coke yield and softening point is the same regardless of the reaction conditions. It is important to note that for these solvents and this type of coal, a coke yield can be predicted from the pitch softening point and is not changed by the reaction conditions of gas composition, gas pressure, solvent-to-coal ratio, and solvent choice. It is not known from this study if the particular linear relationship is dependent on the characteristics of the coal. Further study is warranted in this area.

Table 2.15 Elemental analysis of selected hydrogenation trials as the solvent-to-coal ratio was lowered but all other reaction conditions were kept constant

at

HCO solvent, $400{ }^{\circ} \mathrm{C}, 1000$ psig hydrogen, and 1 hour

\begin{tabular}{|c|c|c|c|c|}
\hline \multirow{2}{*}{$\begin{array}{c}\text { Run } \\
\text { (Solvent- } \\
\text { to-Coal } \\
\text { Ratio) }\end{array}$} & \multirow[b]{2}{*}{ Element } & \multicolumn{3}{|c|}{ Product Fraction } \\
\hline & & Pitch & $\begin{array}{c}\text { THF } \\
\text { Insoluble }\end{array}$ & $\begin{array}{l}\text { Recycle } \\
\text { Solvent }\end{array}$ \\
\hline \multirow{5}{*}{$\begin{array}{c}4 \mathrm{C} \\
(3 / 1)\end{array}$} & C & $91.16 \%$ & $79.02 \%$ & $93.90 \%$ \\
\hline & $\mathrm{H}$ & $5.31 \%$ & $3.96 \%$ & $5.53 \%$ \\
\hline & $\mathrm{N}$ & $1.45 \%$ & $1.42 \%$ & $0.93 \%$ \\
\hline & $\mathrm{s}$ & $0.71 \%$ & $0.96 \%$ & $0.48 \%$ \\
\hline & $\begin{array}{c}\mathrm{H} / \mathrm{C} \text { Atomic } \\
\text { Ratio }\end{array}$ & 0.70 & 0.60 & 0.71 \\
\hline \multirow{5}{*}{$\begin{array}{c}11 \mathrm{~B} \\
(2 / 1)\end{array}$} & C & $89.59 \%$ & $77.53 \%$ & $90.32 \%$ \\
\hline & $\mathrm{H}$ & $5.18 \%$ & $3.95 \%$ & $5.44 \%$ \\
\hline & $\mathrm{N}$ & $1.47 \%$ & $1.70 \%$ & $0.85 \%$ \\
\hline & $\mathrm{s}$ & $0.64 \%$ & $0.71 \%$ & $0.39 \%$ \\
\hline & $\begin{array}{l}\text { H/C Atomic } \\
\text { Ratio }\end{array}$ & 0.69 & 0.61 & 0.72 \\
\hline \multirow{5}{*}{$\begin{array}{l}12 \mathrm{~A} \\
(1 / 1)\end{array}$} & $\mathrm{C}$ & $87.29 \%$ & $76.81 \%$ & $90.46 \%$ \\
\hline & $\mathrm{H}$ & $5.34 \%$ & $4.11 \%$ & $5.74 \%$ \\
\hline & $\mathrm{N}$ & $1.09 \%$ & $1.36 \%$ & $0.66 \%$ \\
\hline & $S$ & $0.64 \%$ & $0.72 \%$ & $0.42 \%$ \\
\hline & $\begin{array}{c}\text { H/C Atomic } \\
\text { Ratio }\end{array}$ & 0.73 & 0.64 & 0.76 \\
\hline
\end{tabular}




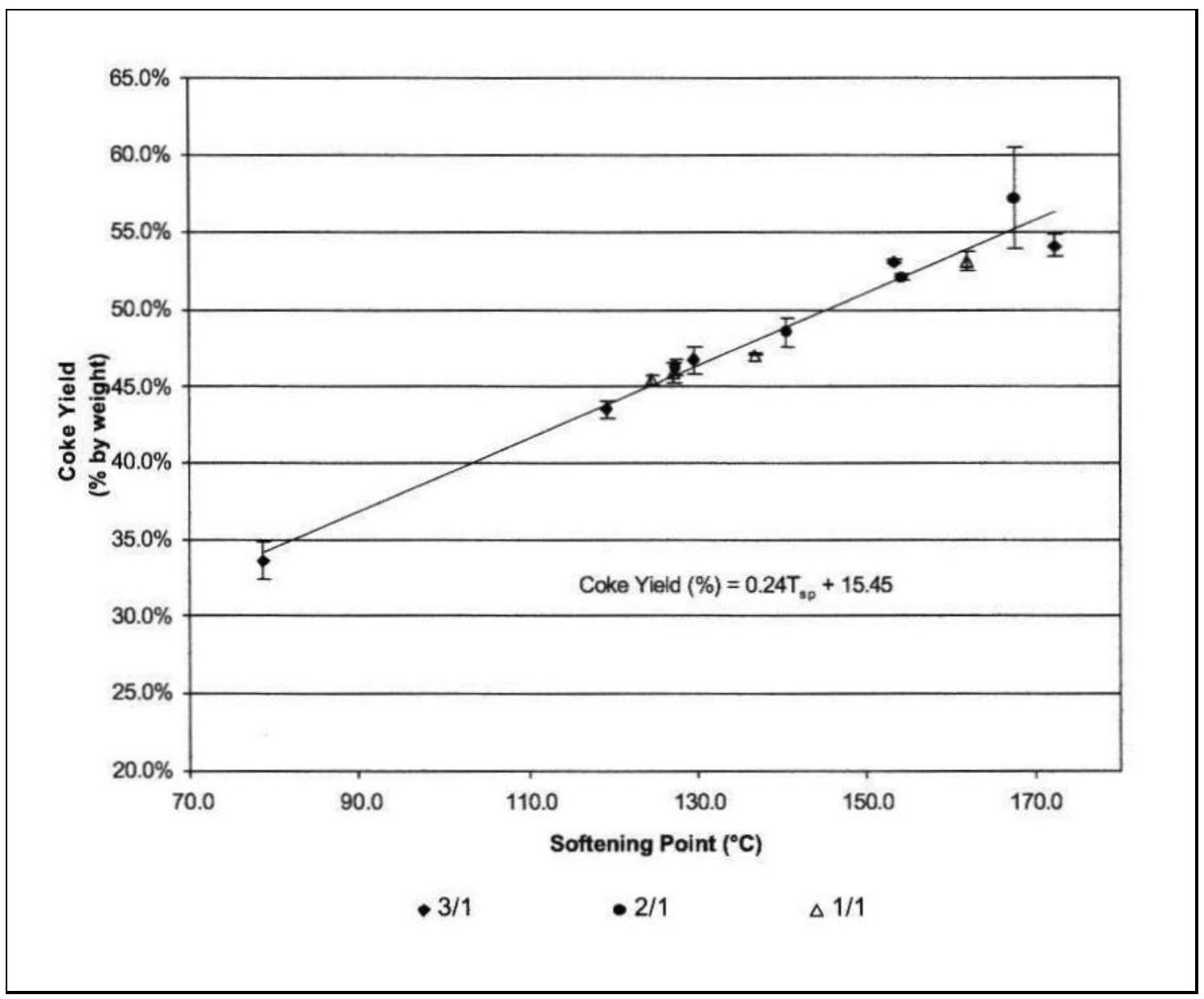

Figure 2.21 Effect of lowering the solvent-to-coal ratio on the pitch coke yield and various softening points from hydrogenation reactions with conditions of HCO solvent, $400{ }^{\circ} \mathrm{C}, 1000$ psig hydrogen, and 1 hour 


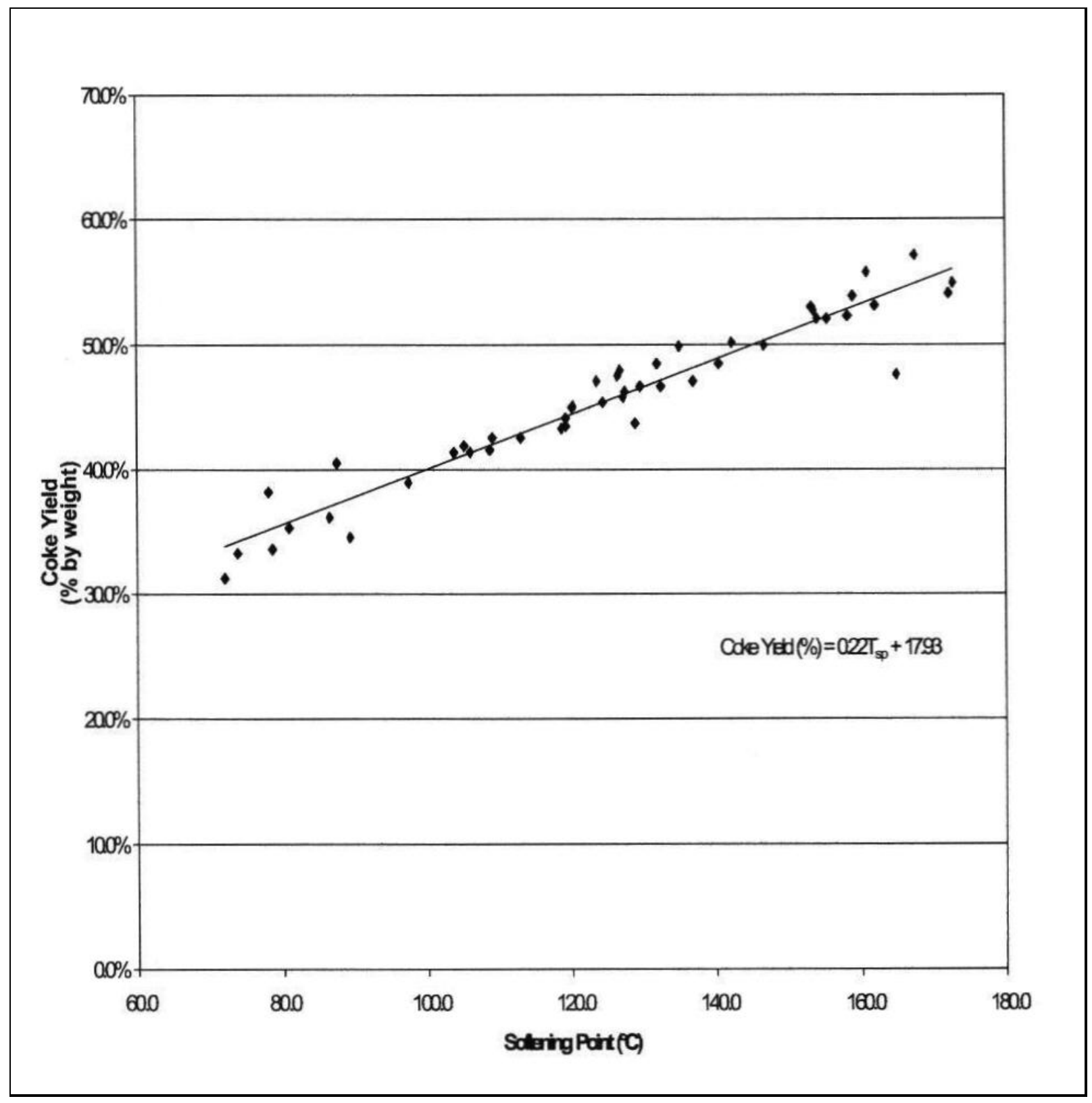

Figure 2.22 Effect of pitch softening point on the coke yield for all pitch fractions produced under varying reaction conditions 


\subsection{Conclusions}

The purpose of this task was to evaluate the effectiveness of five coal-derived liquids as possible hydrogenation solvents for the production of carbon-product precursors from coal. Each of these solvents was employed as a hydrogen donor under constant reaction conditions. The hydrogenation products were then separated into three fractions, pitch, THF insolubles, and recycle solvent. The pitch fraction was then characterized by standard methods to compare its properties to those of commercially available pitches. Finally, some of the reaction conditions were varied (pressure, gas composition, and solvent-to-coal ratio) in order to understand their effect on the products of the hydrogenation reactions. From these experiments several conclusions can be drawn.

1) The overall conversion seems to mask the effect of changing reaction conditions on coal reactivity because of the high amounts of solvent compared to the amount of coal included in the calculation. The coal-alone conversion, on the other hand, gives a relatively clear picture of the effect of changing the reaction conditions.

2) By using the coal-alone conversion, the most effective solvent for creating soluble species from coal was DACL-L (46.7\%) followed by HCO (30.8\%) and CBB $(23.2 \%)$.

3) The coal-alone conversion was also dependent on the initial reaction pressure and gas composition. As the pressure increased, the coal-alone conversion increased for both hydrogen and argon atmospheres but not as significantly for the argon. This leads to the conclusion that the gaseous hydrogen does play an active role in converting the coal to soluble species.

4) The coal-alone conversion did not show a dependence on the solvent-to-coal ratio in the hydrogenation reactions. This is a valuable result in that the least amount of solvent can be employed that physically allows for practical processing. It was found that a solvent-to-coal ratio of $1 / 1$ was too low for adequate processing in these experiments because of the resistance to flow of the reaction products. Therefore, a $2 / 1$ solvent-to-coal ratio is recommended for any subsequent reactions.

5) Once the hydrogenation reactions took place, the products were separated into the THF insolubles, recycle solvent, and the primary product of pitch. This pitch fraction was tested as a possible precursor for carbon products by standard characterization techniques, such as softening point, ash content, coke yield, optical texture, and elemental analysis. The softening point of each pitch could be tailored to a specific need by changing the extent of distillation. Pitches were produced with a softening 
point as low as $70^{\circ} \mathrm{C}$ up to $180^{\circ} \mathrm{C}$ regardless of the reaction conditions utilized.

6) The ash content of all of the product pitches was at an acceptably low value of less than $0.1 \%$. This ash value is lower than that for pitches currently used as binders and is approaching the ash value of impregnation pitches.

7) The effect of the processing conditions on the coke yield of the product pitches was also investigated. The coke yield was nearly linearly dependent on the softening point of the pitch (controlled by extent of distillation) for all reaction conditions. The coke yield of pitches with a softening point near the commercial target value of 120 ${ }^{\circ} \mathrm{C}$, however, was somewhat lower than that desired for commercial applications (45\% compared to $50-60 \%$ ). Although the coke yield was dependent on the extent of distillation, it showed no other dependence on the reaction conditions. Changing the reaction pressure, gas composition, solvent-to-coal ratio, and even solvent did not have a significant effect on the coking value.

8) All pitches produced anisotropic coke regardless of the solvent choice. Anisotropic coke could also be produced at low pressures (100 psig) and without hydrogen. This discovery could significantly reduce the operational costs of pitch production.

9) Two important observations were derived from the elemental analysis of the pitch and recycle solvent fractions. First, the pitch fraction showed a carbon content $(\cong 90 \%)$ similar to that of commercial binder pitches. Also, the elemental composition of the recycle solvent is similar to that of the original solvent. This could lead to a process that generates its own process solvent, thus alleviating the need for adding more solvent.

The above conclusions lead to the recommendation of an optimum process for the production of pitch from coal and coal-derived liquids. The solvent of choice would be HCO since it exhibited the highest coal-alone conversion while being commercially available and produced a pitch which yielded an anisotropic carbon. The solvent-to-coal ratio would be $2 / 1$ for ease of processing, and the pressure and gas composition would be 1000 psig hydrogen to maximize conversion. However, the pressure and gas composition could be changed if the processing costs outweigh that of maximizing the conversion.

\subsection{REFERENCES}

1. A University, Industry, Government Cooperative Research Partnership. "Non-Fuel Use of Coal: Coal-Derived Carbon Materials". Nov. 12, 1993. The National Research Center for Coal and Energy (NRCCE), West Virginia University, P. O. Box 6064, Morgantown, WV 26505-6064.

2. Artok, L., Schobert, H.H., and Erbator, O., Fuel Process. Technol., 37, 221, 1994.

3. ASTM D 2415 Standard Test Method for Ash in Coal Tar and Pitch. 
4. ASTM D 3104 Standard Test Method for Softening Point of Pitches (Mettler Softening Point Method).

5. Berkowitz, N. An Introduction to Coal Technology. Academic Press, Inc., San Diego, 1994.

6. Chaudhuri, P.D., The Production of Ultra-Pure Coke Precursors for the Manufacture of Value-Added Carbon Products. Thesis, Department of Chemical Engineering, West Virginia University, Morgantown, WV, 1994.

7. Chawla, B., Keogh, R., and Davis, B.H., Energy \& Fuels, 3, 236, 1989.

8. $\quad$ Cloke, M. and Wang, C., Energy \& Fuels, 9, 560, 1995.

9. Curran, G. P., Struck, R. T., and Gorin, E., Ind. Eng. Chem. Process Des. Dev., 6, 166, 1967.

10. Edwards, I.A.S., "Structure in Carbons and Carbon Forms." Introduction to Carbon Science. Ed. H. Marsh, Butterworths, 1989.

11. Ergun, S., "Coal Classification and Characterization," Coal Conversion Technology. Ed. C.Y. Wen and E.S. Lee, Addison-Wesley Publishing Company, Reading, Mass., 1979.

12. Farcasiu, M., Mitchell, T. O., and Whitehurst, D. D., ACS Div. Fuel Chem., Prepr. 27(7), 11, 1976.

13. "Final Technical Report" Coal Based Nuclear Graphites for the New Production Gas Cooled Reactor. Task 1: Development of Coal-Derived Isotropic Coke and Nuclear Graphite. West Virginia University, 1994.

14. Finseth, D.H., Cillo, D.L., Sprecher, R.F., Retcofsky, H.L., and Lett, R.G., Fuel, 64, $1718,1985$.

15. Fisher, C. H., Sprunk, G. C., Eisner, A., O’Dennell, H. J., Clarke, L., and Storch, H. H., "Hydrogenation and Liquefaction of Coal," Part 2: "Effect of Petrographic Composition and Rank of Coal," U. S. Bur. Mines Tech. Paper, 642, 1942.

16. Francis, W., Coal - Its Formation and Composition. Edward Arnold (Publishers) Ltd., 1954.

17. Gibson, J., Coal: an Introduction to its Formation and Properties. In Coal and Modern Coal Processing: An Introduction, ed. G. J. Pitt and G. R Millward. Academic Press, New York, 1979. 
18. Given, P. H., Cronauer, D. C., Spackman, W., Lovell, H. L., Davis, A., and Biswas, B., "Dependence of Coal Liquefaction Behavior on Coal Characteristics," Parts I and II, Fuel, 54, 34 and 40, 1975.

19. Gorin, E., "Fundamentals of Coal Liquefaction." Chemistry of Coal Utilization, ed. M.A. Elliott. John Wiley and Sons, New York, 1981, pp. 1845-1918. 20. Huang, H., Wang, K., Wang, S., Klein, M.T., and Calkins, W.H., Energy \& Fuels, 12, 95, 1998.

21. Keogh, R.A. and Davis, B.H., Energy \& Fuels, 8, 289, 1994.

22. Keogh, R.A., Taulbee, D.N., Hower, J.C., Chawla, B., and Davis, B.H., Energy \& Fuels, 6, 614, 1992.

23. Lee, E.S., "Coal Liquefaction.” Coal Conversion Technology. Ed. C.Y. Wen and E.S. Lee, Addison-Wesley Publishing Company, Reading, Mass., 1979.

24. Malhotra, R. and McMillen, D.F., Energy \& Fuels, 4, 184, 1990.

25. Malhotra, R. and McMillen, D.F., Energy \& Fuels, 7, 227, 1993.

26. Merrick, D. Coal Combustion and Conversion Technology. Elsevier: New York, 1984.

27. Mukherjee, D. K., and Chowdry, P. B., Fuel, 55, 4, 1976.

28. Neavel, R. C., Fuel, 55, 237, 1976.

29. Oele, A. P., Waterman, H. I., Goedkoop, M. L., and Van Krevelen, D. W., Fuel, 30, $169,1951$.

30. Orchin, M. and Storch, H. H., Ind. Eng. Chem., 40, 1385, 1948.

31. Schobert, H. Coal: The Energy Source of the Past and Future. American Chemical Society, USA, 1987.

32. Song, C. and Schobert, H. H., Fuel, 1996, 75(6), 724-736.

33. Speight, J.G. The Chemisty and Technology of Coal. Marcel Dekker: New York, 1983.

34. Stansberry, P.G., Zondlo, J.W., and Stiller, A.H., "Coal-Derived Carbons." Carbon for Advanced Technologies. Ed. T.D. Burchell, Pergamon, Amsterdam, 1999.

35. Stopes, M.C., Proc. R. Soc. London, 90B, 470, 1919. 
36. Tomic, J. and Schobert, H.H., Energy \& Fuels, 10, 709, 1996.

37. Van Krevelen, D.W., Coal, Typology-Chemistry-Physics-Constitution. Elsevier Scientific Publishing Company, Amsterdam, 1981.

38. Vernon, L.W., Fuel, 59, 102, 1980.

39. Wen, C.Y. and Dutta, S., "Rates of Coal Pyrolysis and Gasification Reactions." Coal Conversion Technology. Ed. C. Y. Wen and E.S. Lee. Addison-Wesley: Reading, Mass., 1979, pp. 57-170.

40. Whitehurst, D.D., Mitchell, T. O., and Farcasiu, M., Coal Liquefaction: The Chemistry and Technology of Thermal Processes, Mobil Research and Development Corporation, Central Research Division, Princeton, New Jersey, 1980.

41. Williams, D.G. "Coal Cleaning.” Coal Handbook. Ed. R.A. Meyers. Marcel Dekker, Inc., New York, 1981, pp. 209-302.

42. Wilson, M.A., Pugmire, R.J., Vasallo, A.M., Grant, D.M., Collin, P.J., and Zilm, K.W., Ind. Eng. Chem. Prod. Res. Dev., 21, 477, 1982.

43. Wiser, W. Preprints Fuel Division ACS Meeting, 20 (2), 122, 1975.

44. Wiser, W. H., Fuel, 47, 475, 1968.

45. Yang, J., A Study on the Carbonization of Coal-Derived Pitches. Thesis, Department of Chemical Engineering, West Virginia University, Morgantown, WV, 1994.

46. Yarzab, R. F., Given, P.H., Davis, A., and Spackman, W., Fuel, 59, 81, 1980.

47. Yen, Y. K., Furlani, D. E., and Weller, S. W., Ind. Eng. Chem. Prod. Res. Dev., 15, 24, 1976. 


\section{Task 3.0 Laboratory Scale Testing of Alternate Solvents}

\subsection{Introduction}

Currently, the primary source of carbon material is petroleum. Strategic, technical and environmental constraints make it imperative that a new source of this feedstock be obtained. Coal, though attractive in terms of domestic availability and supply, is disadvantageous in that it contains ash and other environmentally sensitive components such as sulfur. However, engineers and scientists of the Carbon Product Program in the Department of Chemical Engineering at West Virginia University have developed a coal pitch extract which is virtually free of ash and other inorganic material; this extract can be used as a precursor for a variety of carbon-based materials. The extract is obtained by using a class of organic solvents known generically as dipolar aprotic compounds. The solvent used for earlier studies was N-methyl-pyrrolidone(NMP). Due to the price and local availability, we are trying to use other solvents to prepare the precursors of carbon materials. To this end, this project task tested and compared the efficacy of NMP, DMAC, DMF, cyclohexanone both neat and mixed with the co-solvent $\mathrm{CS}_{2}$. These solvents were found to have very good extractability for hydrogenated coal, even at room temperature. The extraction yield for hydrogenated coal is $81.5 \%$ for NMP and $62.6 \%$ for DMAC at room temperature. Raw coal is harder to dissolve. The best extraction yield for the selected coal (WVGS13421, bituminous coal) using NMP at its boiling point $\left(202^{\circ} \mathrm{C}\right)$ is $30 \sim 40 \%$. Earlier, additives were reported to enhance coal solubility by breaking coal molecular interaction. The works were focused on $\mathrm{NMP} / \mathrm{CS}_{2}(1 / 1, \mathrm{v} / \mathrm{v})$ mixed solvent. In the current project, we investigated the effect of additives on coal extraction in NMP, DMAC and DMF single solvent system. Thermal dissolution of coal at temperatures up to $400^{\circ} \mathrm{C}$ was also investigated.

\subsection{Experimental}

\subsubsection{Coal Sample}

The investigation of the extraction of coal using alternative solvents was focused on the following coals listed in Table 3.1. The coal was ground to less than 60 mesh and vacuum dried at $80^{\circ} \mathrm{C}$ overnight. The volatile matter and ash content were measured according to ASTM method D5142 and D3174, respectively. The organic elemental composition was measured by CE Instruments EA1112 elemental analyzer. The results of these analyses for the three coals used in this work are listed in Table 3.1.

\section{Table 3.1. Proximate and ultimate analysis of coals}




\begin{tabular}{|c|c|c|c|c|c|c|c|c|}
\hline \multirow[t]{2}{*}{ Coals } & \multirow[b]{2}{*}{ Location } & \multirow{2}{*}{$\begin{array}{c}\mathrm{VM} \\
\mathrm{wt} \%, \mathrm{db}\end{array}$} & \multirow{2}{*}{$\begin{array}{c}\text { Ash } \\
\mathrm{wt} \%, \mathrm{db}\end{array}$} & \multicolumn{5}{|c|}{ Elemental analysis, wt $\%(\mathrm{daf})$} \\
\hline & & & & $\mathrm{C}$ & $\mathrm{H}$ & $\mathrm{N}$ & $\mathrm{S}$ & $\mathrm{O}^{*}$ \\
\hline WVGS13421 & WV & 29.19 & 6.20 & 84.91 & 4.87 & 1.75 & 1.34 & 7.14 \\
\hline PSOC3001 & PA & 31.57 & 7.36 & 86.69 & 4.92 & 1.52 & 0.766 & 6.10 \\
\hline WVSC001 & WV & 39.14 & 4.33 & 81.01 & 5.37 & 1.95 & 1.25 & 10.43 \\
\hline
\end{tabular}

*by difference

\subsubsection{Solvents and Chemicals}

The extraction solvents used include N-methyl-2-pyrrolidone (NMP), N, NDimethylformamide(DMF), N, N-Dimethylacetamide (DMAC), Cyclohexanone, and $\mathrm{CS}_{2}$. These solvents are industrial grade and used as received without further purification. The additives used to enhance coal extraction include Tetrabutylammonium acetate(TBAA), Tetramethylammonium acetate(TMAA), Ferric Chloride, 1, 4 Phenylenediamine( $p$-PDA), Chlorine, 2, 3-Dichloro-5, 6-dicyano-1, 4-benzoquinone(DDQ), Quinone, 7, 7, 8, 8Tetracyanoquinondimethane(TCNQ) and Ethylenediamine(EDA). These additives were purchased from Aldrich Co. and were used as received.

\subsubsection{Solvent Extraction}

The extraction at or below solvent's boiling point was carried out in a $250 \mathrm{ml}$ KIMAX flatbottom boiling flask with a reflux condenser (if necessary) and magnetic stirrer. Coal/solvent ratio is $1 / 10(\mathrm{w} / \mathrm{v})$, e.g., $10 \mathrm{~g}$ coal and $100 \mathrm{ml}$ solvent. The residues and extract solution were separated by centrifugation (4000rpm, $1 \mathrm{hr}$.) and filtration(filter paper, Whatman \#1, $\sim 11 \mu \mathrm{m})$. The residue was washed by fresh extraction solvent once, followed by ane-time acetone wash. Extract was collected by evaporation of solvent in a rotary evaporator. Both extracts and residues were vacuum dried at $170^{\circ} \mathrm{C}$ overnight.

The extraction yield is based on daf coal, using the formula below:

[(weight of raw coal, db)-(weight of residue, $\mathrm{db})]$

Extraction yield, $\mathrm{wt}^{\mathrm{O}} \mathrm{=}=\frac{}{[(\text { weight of raw coal }, \mathrm{db}) \times(1-\mathrm{ash}, \mathrm{wt} \%)]} \times 100 \%$

\subsubsection{Hydrogenation of Coal}


Hydrogenation was used to adjust the coal extract properties to meet the requirement of fiber spinning and making carbon foam. The details about hydrogenation were described in Task 2. The hydrogenation conditions used for preparation of $\mathrm{H}$-coal in this experiment were initial $\mathrm{H}_{2}$ pressure, $400 \mathrm{psi}$, hydrogenation temperature $400^{\circ} \mathrm{C}$ for $1 \mathrm{hr}$ in tetralin without a catalyst. Hydrogenated coal and tetralin were separated by rotary evaporation and the hydrogenated coal was then vacuum dried overnight.

\subsubsection{Thermal Dissolution of Coal}

Thermal dissolution of coal includes a heat treatment of coal at elevated temperature followed by a solvent extraction of the treated coal. The medium used for heat treatment and solvent extraction could be the same or different. The following are the two methods investigated in this project.

\subsubsection{NMP as the Heat Treatment Medium and Extraction Solvent}

Coal and NMP $(1 / 10, \mathrm{~g} / \mathrm{mL})$ were mixed and sealed in a $120 \mathrm{~mL}$ bomb reactor and charged with $\mathrm{N}_{2}$ to 200psi. The reactor was heated in a sand bath at the selected temperature for one hour with agitation. After the heat treatment, the contents of the reactor were transferred to a flask and refluxed in NMP for 0.5 hour. The extract solution and the residue were then separated by centrifugation and filtration; and the extraction yield based on the weight of the residue was determined.

\subsubsection{Creosote Oil or Coal-derived Pitch as the Heat Treatment Medium and NMP as the Extraction Solvent}

Coal and creosote oil(or coal derived pitch)(1/1.5, g/g) were mixed and sealed in a $120 \mathrm{~mL}$ bomb reactor(without $\mathrm{N}_{2}$ charge) and heated in a sand bath at the selected temperature $\left(350^{\circ} \mathrm{C}\right.$ in this work) for one hour(no agitation). After the heat treatment, the contents of the reactor were transferred to a flask and refluxed in NMP for 0.5 hour. The extract solution and the residue were then separated by centrifugation and filtration and the extraction yield based on

the weight of the residue was determined.

\subsubsection{Kinetics of Coal Swelling in the Solvents}

Raw coal was ground and dried at $100^{\circ} \mathrm{C}$ under vacuum over night. To minimize the effect of particle size on the swelling of coal, the particle size of 60 to $140 \mathrm{mesh}(\mathrm{e} . \mathrm{g}$., 106 to $250 \mu \mathrm{m}$ ) was selected. A flat-bottom glass tube with a $5 \mathrm{~mm}$ i.d. and $120 \mathrm{~mm}$ length sealed 
at one end was used for swelling. About $0.3 \mathrm{~g}$ ground coal was placed in the swelling tube and was centrifuged for 5 minutes at $2500 \mathrm{RPM}$. The initial height $\left(\mathrm{H}_{1}\right)$ of the coal-bed in the glass tube was measured. Approximately 2-3 ml solvent was added into the tube at time $\mathrm{t}=0$. Coal and solvent were thoroughly mixed using a thin metal rod. The sample was centrifuged at 2500 RPM for 5 minutes and the height of coal bed $\left(\mathrm{H}_{2}\right)$ was measured at appropriate time, $\mathrm{t}$, which is taking into account the time for centrifugation. The volumetric swelling ratio $(\mathrm{Q})$ is defined as, $\mathrm{Q}=\mathrm{H}_{2} / \mathrm{H}_{1}$.

\subsubsection{Characterization of Coal and Coal Extracts}

The raw coal, hydrogenated coal and coal extracts were characterized by elemental analysis and FTIR. The elemental analyzer is a CE Instruments EA1112 C, H, N, S analyzer. Sample weight range is about $2 \mathrm{mg}$. FTIR was carried out on a Nicolet 510P FTIR spectrometer. The diffuse reflection method was employed.

\subsection{Results and Discussion}

\subsubsection{Extraction of Raw Coal}

Three bituminous coals were selected for the solvent extraction. The extraction yield of these coals using NMP, DMAC and DMA is listed in Table 3.2. The values in the parentheses behind the extraction yield is the mass balance for the extraction, represented as wt $\%$ recovery.

NMP, DMF and DMAC have similar structure as shown below, but their extractability for the coal is different. The results indicate that NMP is more effective than the other two solvents. DMAC is better than DMF, but both their extraction yields for the three coals are very close. DMAC and DMF are stronger bases than NMP, but they do not show a better extractability. It is clear that basicity alone cannot explain the observed relative difference in extractability. The relation between solvent properties and coal extraction is not totally understood. Some researchers suggest that the viscosity of the solvent [1-2], the proportion of monomers of solvent molecules [2-4] and the ability to break aggregated coal molecules [5] affect the solvent extraction. Based on this research and the literature, molecules containing $\mathrm{N}$ and $\mathrm{O}$ would be a good extractant.

Table 3.2 Extraction yield of raw coal using various solvents*

\begin{tabular}{|l|c|c|c|}
\hline \multirow{2}{*}{ Coals } & \multicolumn{2}{|c|}{ Extraction yield, wt\%(based on daf coal) } \\
\cline { 2 - 4 } & NMP & DMF & DMAC \\
\hline WVGS13421 & $35.9(104.7)^{* *}$ & $6.7(100.3 \%)$ & $9.4(100.8 \%)$ \\
\hline PSOC3001 & 33.3 & $15.0(98.6 \%)$ & $20.0(101 \%)$ \\
\hline
\end{tabular}




\begin{tabular}{|l|c|c|c|}
\hline & & $16.9(99.3 \%)$ & $20.0(102 \%)$ \\
\hline WVSC001 & 26.2 & 17.1 & 20.3 \\
& 25.2 & 17.4 & 20.6 \\
& 24.0 & & \\
\hline
\end{tabular}

* Refluxed at boiling point of the solvent for $1 \mathrm{hr}$.

** The values in the parentheses are the recovery $w t \%(d b)$, i.e., $[($ residue + extract $)(\mathrm{g}) / \mathrm{coal}(\mathrm{g})] \times 100 \%$<smiles>CN1CCCC1=O</smiles>

NMP

b.p. $202^{\circ} \mathrm{C}$<smiles>CN(C)C=O</smiles>

DMF

$153^{\circ} \mathrm{C}$<smiles>CC(=O)N(C)C</smiles>

DMAC

$166^{\circ} \mathrm{C}$

In addition to the solvent structure, coal properties and structure also affect coal extraction. It has been reported that bituminous coal with carbon content around $80 \sim 86 \%$ shows better extraction yield in NMP, DMF and DMAC, etc. The three coals selected here are bituminous coal with similar elemental composition, but their extractability is different. At present, we are unable to make any conclusion about their different extractability based on the limited knowledge about coal and solvent.

\subsubsection{Extraction of Hydrogenated Coal}

Hydrogenation greatly changes the solubility of coal and the properties of coal extracts. The coal (WVGS13421) was hydrogenated in tetralin with an initial cold hydrogen pressure of 400psi and hydrogenated at $400^{\circ} \mathrm{C}$ for $1 \mathrm{hr}$, without a catalyst. This mild hydrogenation causes less than $1 \%$ hydrogen to be transferred to the coal [6]. It is not deep enough to convert coal to fuel oil, but it is enough to tailor the properties of hydrogenated coal extract, i.e., so called "coal-derived pitch". The comparison of extraction yield of raw coal and hydrogenated coal is shown in Table 3. 3. There is a significant increase of the extraction yield of hydrogenated coal over the raw coal, especially for extraction using DMAC and DMF. It is also noticed that the mass balance of hydrogenated coal is lower than $100 \%$. It 
may be caused by the loss of retained tetralin in the hydrogenated coal, which was not completely removed by vacuum evaporation during the preparation of hydrogenated coal, but further removed during the vacuum oven drying of the extract and residue.

Table 3.3 Effect of hydrogenation on the solubility of coal (WVGS13421) in the solvents. Extraction is at the boiling point of the solvent for $1 \mathrm{hr}$.

\begin{tabular}{|l|c|c|c|c|}
\hline \multirow{2}{*}{ Solvents } & \multicolumn{2}{|c|}{ Raw coal } & \multicolumn{2}{c|}{ Hydrogenated coal } \\
\cline { 2 - 5 } & $\begin{array}{c}\text { Extraction yield, } \\
\mathrm{wt} \%, \mathrm{daf}\end{array}$ & $\begin{array}{c}\text { Mass balance* } \\
\mathrm{wt} \%, \mathrm{db}\end{array}$ & $\begin{array}{c}\text { Extraction yield } \\
\mathrm{wt} \%, \mathrm{daf}\end{array}$ & $\begin{array}{c}\text { Mass balance* } \\
\mathrm{wt} \%, \mathrm{db}\end{array}$ \\
\hline NMP & 35.9 & 104.6 & 85.3 & 96.3 \\
\hline DMAC & 9.4 & 100.8 & 70.7 & 96.6 \\
\hline DMF & 6.7 & 100.3 & 51.8 & 96.4 \\
\hline
\end{tabular}

$*[($ extract + residue $)(\mathrm{g}) / \operatorname{coal}(\mathrm{g})] \times 100 \%$

Hydrogenated coal even shows very high extraction yield at room temperature. Table 3. 4 lists the comparison of the extraction yield of hydrogenated coal extracted at room temperature and at the solvent's boiling point. For hydrogenated coal, the NMP extraction yield is nearly identical at room temperature and at the solvent's boiling point and temperature has little effect on the NMP extraction. However, temperature greatly affects the extraction using the other three solvents, i.e., the extraction yield at the respective boiling point of DMAC, DMF and cyclohexanone is much higher than that at room temperature.

Table 3. 4 Comparison of the extraction yield of hydrogenated coal* using different solvents.

\begin{tabular}{|l|c|c|c|}
\hline & \multirow{2}{*}{$\begin{array}{c}\text { Boiling point of } \\
\text { Solvent, }{ }^{\circ} \mathrm{C}\end{array}$} & \multicolumn{2}{|c|}{$\begin{array}{c}\text { Extraction yield, wt\% } \\
\text { (based on daf coal) }\end{array}$} \\
\cline { 3 - 4 } & & Room temp. & Refluxing at b.p. \\
\hline NMP & 202 & 81.5 & 85.2 \\
\hline DMAC & 166 & 62.6 & 70.7
\end{tabular}




\begin{tabular}{|l|c|c|c|}
\hline DMF & 153 & 38.1 & 51.6 \\
\hline Cyclohexanone & 155 & 37.5 & 66.5 \\
\hline
\end{tabular}

*WVGS13421 hydrogenated coal, $400^{\circ} \mathrm{C}, 400$ psi of $\mathrm{H}_{2}$ (cold) for $1 \mathrm{hr}$ in tetralin.

The effect of hydrogenation on coal extraction is attributed to the breaking of covalent bond linkages and the loss of the oxygen compounds, which may play a key role in formation of the non-covalent coal molecular interactions. The loss of oxygen is confirmed by the elemental analysis results listed in Table 3. 5, which indicate that oxygen content is greatly reduced in the hydrogenated coal and its extract. Although $\mathrm{H} / \mathrm{C}$ atomic ratio is increased for the hydrogenated coal. It is not possible to compare between the raw coal extracts and the hydrogenated coal extracts because of the solvent retention, especially for the raw coal extracts.

Table 3.5 Elemental analysis of raw coal, hydrogenated coal and their NMP extracts

\begin{tabular}{|l|c|c|c|c|c|c|}
\hline \multirow{2}{*}{ Samples } & \multicolumn{5}{|c|}{ Elemental composition, wt\%(daf) } & \multirow{2}{*}{ H/C } \\
\cline { 2 - 6 } & $\mathrm{C}$ & $\mathrm{H}$ & $\mathrm{N}$ & $\mathrm{S}$ & O* & \\
\hline WVGS13421 raw coal & 84.91 & 4.87 & 1.75 & 1.34 & 7.13 & 0.689 \\
\hline WVGS13421 H-coal & 89.99 & 5.71 & 1.79 & 1.33 & 1.18 & 0.761 \\
\hline Raw coal extract & 82.53 & 5.27 & 2.58 & 1.16 & 8.46 & 0.766 \\
\hline H-coal extract & 89.05 & 5.58 & 2.02 & 1.74 & 1.61 & 0.752 \\
\hline
\end{tabular}

*by difference

\subsubsection{Synergistic Effect of $\mathrm{CS}_{2}$}

$\mathrm{NMP} / \mathrm{CS}_{2}(1: 1, \mathrm{v} / \mathrm{v})$ mixed solvent was reported to be a good extractant for some bituminous coal, especially for those with carbon content around 86\%, such as Upper Freeport coal (USA) and Zaozhuang coal (China) [1]. The extraction yields for these coals are over $60 \%$. The advantage of this mixed solvent is that the extraction can be carried out at room temperature. However, $\mathrm{CS}_{2}$ is dangerous to handle on an industrial scale. So, this mixed solvent is not recommended for industrial utilization.

Table 3.6 lists the extraction yield of NMP/CS 2 mixed solvent for a variety of WV and PA coals. WVGS13421 and PCOS3001 show the better extractability. 
Table 3. 6 Extraction yield of $\mathrm{NMP} / \mathrm{CS}_{2}$ mixed solvent for selected West Virginia and Pennsylvania bituminous coal. Extraction carried out at room temperature.

\begin{tabular}{|l|c|}
\hline Coals & Extraction yield, wt $\%$ (daf) \\
\hline WVGS13421 & 22.0 \\
\hline WVGS13425 & 17.4 \\
\hline WVGS13426 & 15.4 \\
\hline PSOC3001 & 22.6 \\
\hline WVSC001 & 14.5 \\
\hline
\end{tabular}

To compare the extraction behavior using this mixed solvent with the single solvent, raw coal and its hydrogenated coal were extracted by NMP, NMP/CS $\mathrm{CS}_{2} ; \mathrm{DMAC}, \mathrm{DMAC} / \mathrm{CS}_{2}$; $\mathrm{DMF}, \mathrm{DMF} / \mathrm{CS}_{2}$ and cyclohexanone, cyclohexanone/CS . The results are shown in Table 3.7. The $\mathrm{CS}_{2}$ mixed solvents show higher extraction yield, suggesting a significant synergistic effect of $\mathrm{CS}_{2}$. The reason for this effect is not clear yet, but it was reported to be related to the monomer of NMP molecules, i.e., $\mathrm{CS}_{2}$ is able to lower the aggregation state of the NMP and increase the proportion of NMP monomers [4].

\subsubsection{Effect of Ultrasonic Radiation on Extraction}

Ultrasonic radiation was thought to provide energy to break some weak molecular interactions in coal. [7-8]. The effect of ultrasonic radiation or mechanical stirring (magnetic stirring here) on extraction were compared in Table 3. 8. No significant difference was observed in this research.

\subsubsection{Effect of Additives}

\subsubsection{Effect of Additives on the Extraction Behavior}

It has been reported that trace amounts of additive can significantly increase the extraction yield of coal in the NMP/CS 2 (1:1, v/v) mixed solvent [9-12]. These additives include TCNE, TCNQ, Tetrabutylammonium acetate, $p$-PDA, etc. However, the $\mathrm{CS}_{2}$ solvent system is not industrially attractive. Therefore, in this project, the effect of these additives in NMP and other single solvent systems was investigated. 
Eight additives were investigated. Table 3.9 lists the effect of these additives on the extraction yield of NMP, DMF and DMAC. The results indicated that the anionic additive, such as the acetate and chloride salt, and EDA are effective. However, some additives are not effective, and even cause a negative effect. These additives include $p$-PDA, DDQ, Chlorine, etc.

Both our research and the literature data $[3,10]$ indicate that anionic additives are effective. The anionic enhancement of the extraction is probably because they may induce a polarization in the aromatic system in coal, leading to disruption of low energy interaction, such as $\pi-\pi$ and van der Waals forces $[3,10]$. The dissolution of coal could be considered as an equilibrium between the strong aggregated coal molecules (undissolved part) and the less aggregated coal molecules(dissolved part) in the solution. During the solvent extraction of coal in the presence of anionic additives, the anionic additives break the non-covalent interaction between coal molecules, while also preventing the coal molecules from aggregating back to a stronger state.

Two acetate salts, Tetrabutylammonium acetate and Tetramethylammonium acetate, are found to be effective. Although the additive will be retained in the extract, it can be burned out during the carbonization. So, the acetate salt seems to be an ideal additive. Ferric chloride is also effective, but it is not suitable for making the precursor of carbon products and clean fuel, since it will remain in the extract and increase the ash content of the extract. 
Table 3.7 Comparison of the extraction yield using pure solvent and $\mathrm{CS}_{2}$ mixed solvent. Extraction carried out at room temperature for $1 \mathrm{hr}$.

\begin{tabular}{|c|c|c|c|c|c|c|c|c|}
\hline \multirow[b]{2}{*}{ Coals } & \multicolumn{8}{|c|}{ Solvents } \\
\hline & NMP & $\begin{array}{l}\mathrm{NMP} / \mathrm{CS}_{2} \\
(1: 1, \mathrm{v} / \mathrm{v})\end{array}$ & DMAC & $\begin{array}{c}\mathrm{DMAC} \mathrm{CS}_{2} \\
(1: 1, \mathrm{v} / \mathrm{v})\end{array}$ & $\mathrm{DMF}$ & $\begin{array}{l}{\mathrm{DMF} / C S_{2}} \\
(1: 1, \mathrm{v} / \mathrm{v})\end{array}$ & Cyclohexanone & $\begin{array}{c}\text { Cyclohexanone/CS } \\
(1: 1, \mathrm{v} / \mathrm{v})\end{array}$ \\
\hline WVGS13421 & $\begin{array}{l}2.1 \\
1.8\end{array}$ & 22.0 & $\begin{array}{l}1.2 \\
1.4\end{array}$ & $\begin{array}{c}17.9(99.1)^{*} \\
17.3(99.0)\end{array}$ & $\begin{array}{l}1.4 \\
1.4\end{array}$ & $\begin{array}{l}15.8(97.5) \\
16.0(98.0)\end{array}$ & - & - \\
\hline H-WVGS13421 & $\begin{array}{l}80.6 \\
81.5\end{array}$ & $84.9(97.6)$ & 62.6 & $83.7(98.0)$ & 38.1 & $\begin{array}{c}81.4 \\
82.8(95.6)\end{array}$ & 37.5 & $\begin{array}{l}77.9(96.9) \\
79.0(95.6)\end{array}$ \\
\hline WVSC001 & 10.0 & 14.5 & 8.5 & 17.1 & 7.8 & 17.0 & & \\
\hline
\end{tabular}

*The values in the parentheses are the recovery $w t \%(d b)$, i.e., $[($ residue + extract $)(g) / \operatorname{coal}(g)] \times 100 \%$ 
Table 3.8 Comparison of the effect of ultrasonic radiation and magnetic stirring on the extraction yield. Coal, WVGS13421. Extraction at room temperature for $1 \mathrm{hr}$.

\begin{tabular}{|c|c|c|}
\hline \multirow{2}{*}{ Solvent } & \multicolumn{2}{|c|}{ Extraction yield, wt\% } \\
\cline { 2 - 3 } & Ultrasonic, 30min & Mechanic stirring, 30min \\
\hline $\mathrm{NMP} / \mathrm{CS}_{2,}(1: 1, \mathrm{v} / \mathrm{v})$ & 22.7 & 22.2 \\
\hline $\begin{array}{c}\mathrm{NMP} / \mathrm{CS}_{2},(1: 1, \mathrm{v} / \mathrm{v}) \text { with } 0.2 \mathrm{mmole} / \mathrm{g}-\mathrm{coal} \\
\text { Tetrabutylammonium acetate }\end{array}$ & 34.5 & 30.1 \\
\hline
\end{tabular}

EDA, a strong base, is a good solvent for brown coal. It reacts with brown coal by cleaving the ester bond [13]. It increases the NMP extraction yield of WVGS13421 up to $60 \%$ by addition of 1\% EDA in NMP, but NMP is found difficult to remove from the extract. The mass balance (presented as recovery, i.e., ([(extract + residue $)(\mathrm{g})] / \mathrm{coal}(\mathrm{g})] \times 100 \%)$ is $117 \%$, which is much higher than usual, indicating a strong NMP retention. The elemental analysis results indicate that nitrogen content is very high in the extract (see Table 3.10), suggesting strong EDA and NMP retention in the extract. Although the boiling point of EDA is only $117^{\circ} \mathrm{C}$, it is hard to remove even at $170^{\circ} \mathrm{C}$, under vacuum over night. It is not clear whether EDA reacts with these coals or not. Some researchers claimed that no chemical reaction occurred between bituminous coal and primary aliphatic amines [14-15], but more evidence seems to be required to clarify this issue.

Table 3.9 Effect of additives* on the extraction yield (wt\%) of WVGS13421 coal 
in various solvents(extraction at the boiling point of the solvent)

\begin{tabular}{|c|c|c|c|}
\hline \multirow[b]{2}{*}{ Additives } & \multicolumn{3}{|c|}{ Extraction yield, wt $\%$ (based on daf coal) } \\
\hline & NMP & DMAC & DMF \\
\hline without additive & $35.9(104.9)^{*}$ & $\begin{array}{r}13.0(100.0) \\
13.3(99.8)\end{array}$ & $\begin{array}{l}8.51(99.9) \\
8.45(100.1)\end{array}$ \\
\hline Tetrabutylammonium acetate & 50.0 & & \\
\hline Tetramethylammonium acetate & $\begin{array}{l}41.6(100.3) \\
41.3(100.4) \\
41.8(99.8)\end{array}$ & $\begin{array}{l}22.0(101.1) \\
18.0(101.7)\end{array}$ & $\begin{array}{l}13.3(99.7) \\
13.1(99.1)\end{array}$ \\
\hline Ferric Chloride & 51.3 & & \\
\hline Chlorine & 19.4 & & \\
\hline $\begin{array}{l}\text { 2,3-Dichloro-5,6-dicyano-1,4- } \\
\text { benzoquinone(DDQ) }\end{array}$ & 30.0 & & \\
\hline p-PDA & $23.3(104.0)$ & 13.2(101.4) & $\begin{array}{l}9.1(100.9) \\
8.7(101.7)\end{array}$ \\
\hline TCNQ & $31.6(101.8)$ & $9.8(103.3)$ & $7.5(103.1)$ \\
\hline $1 \% \mathrm{EDA}$ & $\begin{array}{c}60.6(117.5) \\
66.4\end{array}$ & & \\
\hline
\end{tabular}

*addition of $0.2 \mathrm{mmole} / \mathrm{g}$-coal

** The values in the parentheses are the recovery wt\%, i.e., $[($ residue + extract $)(\mathrm{g}) / \mathrm{coal}(\mathrm{g})] \times 100 \%$

TCNQ is very effective in NMP/CS 2 mixed solvent, but not in NMP alone. It has been reported that the effect of TCNQ is related to the TCNQ radical anion [16]. The UV spectroscopic investigation indicated that TCNQ generates less radical anions in NMP and pyridine in the absence of $\mathrm{CS}_{2}$ [16]. This may be the reason that TCNQ appears to be 
ineffective in NMP alone. DMAC and DMF have similar structure to NMP. It is not surprising that TCNQ does not promote their enhanced extractability for coal.

$p$-PDA, Chlorine, 2, 3-Dichloro-5, 6-dicyano-1, 4 benzoquinone (DDQ) are ineffective, and even show some negative effects. It is believed that they induce some stronger associations between coal molecules.

Tetrabutylammonium acetate(TBAA) is an effective additive for raw coal extraction. However, it did not seem to be effective for the hydrogenated coal NMP extraction, but it is still effective for DMAC extraction, see Table 3.11 for the details. This is probably because the high solubility of hydrogenation coal in NMP masks the effect of the additive. TBAA does not show any effect on the cyclohexanone extraction, even though its extraction yield for hydrogenated coal is only $34.4 \%$. The results may indicate that the additive needs to be matched with the solvent to achieve its effect; in other words, the effect of the additive needs the synergistic effect of the solvent. 
Table 3.10 Elemental analysis of coal and its extracts and residues from NMP extraction

\begin{tabular}{|l|c|c|c|c|c|}
\hline \multirow{2}{*}{ Samples } & \multicolumn{5}{|c|}{ Elemental composition, wt\%, daf } \\
\cline { 2 - 6 } & $\mathrm{N}$ & $\mathrm{C}$ & $\mathrm{H}$ & $\mathrm{S}$ & $\mathrm{O}^{*}$ \\
\hline WVGS13421 raw coal & 1.75 & 84.91 & 4.87 & 1.34 & 7.13 \\
\hline WVGS13421 NMP Extracts, & 2.58 & 82.53 & 5.27 & 1.16 & 8.46 \\
$202^{\circ} \mathrm{C}$ & & & & & \\
\hline WVGS13421 NMP Residues, & 2.31 & 81.23 & 4.19 & 1.53 & 10.74 \\
$202^{\circ} \mathrm{C}$ & & & & & \\
\hline WVGS13421 NMP/1\% EDA, & 5.88 & 76.00 & 5.16 & 0.976 & 11.98 \\
Extracts, 202 ${ }^{\circ} \mathrm{C}$ & & & & & \\
\hline WVGS13421 NMP/1\% EDA, & 2.44 & 87.09 & 4.78 & 1.51 & 4.19 \\
Residues, 202 ${ }^{\circ} \mathrm{C}$ & & & & & \\
\hline
\end{tabular}

*by difference

\subsubsection{Effect of Additives on Pitch Properties.}

Some additives do not increase, but decrease the extraction yield. The negative effect may be due to the stronger interactions induced by the additives. In this work, it is also found that these additives could be used to manipulate the softening point of the resulting coal-tar pitch extract.

Three quinone substitued compounds were investigated (see the structures shown below). The additive was first dissolved with conventional coal-tar pitch in NMP by refluxing, and then NMP was removed by evaporation. The coal-tar pitch containing the additive appears to exhibit a higher softening point than the original one. Table 3.12 lists the effect of the three additives on the coal-tar pitch softening point and coke yield. The results indicate that at even these very low concentration the additive has a powerful effect on the softening point of the pitch. However, very little change was observed in the coke yield.

Table 3.11 Effect of additive* on the solvent extraction yield of hydrogenated coal 
(WVGS13421). Extraction carried out at room temperature.

\begin{tabular}{|l|l|c|}
\hline Solvent & Additive & Extraction yield, wt \% \\
\hline NMP & None & 85.2 \\
\hline NMP & Tetrabutylammonium acetate(TBAA) & 85.1 \\
\hline DMAC & None & 62.6 \\
\hline DMAC & Tetrabutylammonium acetate(TBAA) & 77.4 \\
\hline DMAC & $p$-PDA & 62.0 \\
\hline Cyclohexanone & None & 37.5 \\
\hline Cyclohexanone & Tetrabutylammonium acetate(TBAA) & 34.4 \\
\hline
\end{tabular}

*addition of $0.2 \mathrm{mmole} / \mathrm{g}$-coal.<smiles>O=C1C=CC(=O)C=C1</smiles>

quinone<smiles>O=C1C(Cl)=C(Cl)C(=O)C(Cl)=C1Cl</smiles>

chlorine<smiles>CC1=C(N)C(=O)C(Cl)=C(Cl)C1=O</smiles>

DDQ

DDQ was reported to form a charge-transfer complex between aromatics [17]. The results in Table 3.12 indicate that $\mathrm{Cl}$ and $\mathrm{CN}$ substituted quinones affect more significantly the softening point, which indicates that they may induce stronger molecular interactions among the pitch molecules.

\subsubsection{Thermal Dissolution of Coal.}

In addition to the extraction of coal using alternative solvent, the thermal dissolution of coal in several solvents at elevated temperature was also investigated. Thermal dissolution of coal includes a heat treatment of coal at elevated temperature, followed by a solvent extraction. The medium used for heat treatment and solvent extraction could be the same or different.

We have examined NMP, creosote oil and coal-derived pitch (NMP extract of hydrogenated coal) as the medium for the thermal dissolution. The advantage of using NMP as the heat 
treatment medium and the extractant is that coal can be directly dissolved into the NMP. However, a high-pressure reactor is required to maintain the NMP as liquid when the operating temperature is up to $400^{\circ} \mathrm{C}$. Creosote oil has higher boiling point range (around $350 \sim 500^{\circ} \mathrm{C}$ ). Therefore, when the thermal treatment is carried out in creosote oil or in coalderived pitch at $300-400^{\circ} \mathrm{C}$, the pressure in the reactor will not be very much greater than atmospheric pressure. This feature can lower the process requirements and the cost for the equipment. The disadvantage of using creosote oil or coal derived pitch as a medium is, after the heat treatment, the treated coal needs a further extraction using NMP or similar solvent to obtain a mineral-free product.

The coal conversion yield (conversion to NMP solubles) for the three coals is listed in Table 3.13. The results indicate that the heat treatment temperature significantly affects the extent of coal conversion. $60 \sim 80 \%$ of coal was converted to a mineral-free carbon product precursor at temperatures between $350 \sim 400^{\circ} \mathrm{C}$.

PSOC3001 was heat treated in various media, e.g., NMP, creosote oil and coal-derived pitch, respectively. It is noted that using creosote oil or coal-derived pitch converts less coal to the NMP soluble product. NMP is unable to donate any hydrogen to coal. The creosote oil and coal-derivated pitch contain some hydro-aromatics rings. It was thought that they may have some hydrogen donation property by de-hydrogenation at high temperature. However, using creosote oil and coal-derived pitch as the heat-treatment medium does not show a higher conversion yield. Therefore, the effect of thermal dissolution may not be due to the hydrogen transfer from solvent. 
Table 3.12 Effect of some additives on coal tar pitch's properties

\begin{tabular}{|c|c|c|}
\hline Sample & Softening Point, & Coke yield, \\
& ${ }^{\circ} \mathrm{C}$ & $\mathrm{wt} \%$ \\
\hline Coal Tar Pitch(CTP) & 110.7 & 63.0 \\
\hline CTP +0.02 mmole Chloranil/g CTP & 123.6 & 62.3 \\
\hline+0.2 mmole Chloranil/g CTP & 174.7 & 64.5 \\
\hline CTP + 0.02 mmole DDQ/g CTP & 120.4 & 62.1 \\
\hline+0.2 mmole DDQ/g CTP & 143.0 & 64.6 \\
\hline CTP + 0.2 mmole Quinone/g CTP & 113.7 & 61.2 \\
\hline & & 61.2 \\
\hline
\end{tabular}

\section{Table 3.13 Coal conversion yield of thermal dissolution of coal, wt $\%$}




\begin{tabular}{|c|c|c|c|c|c|}
\hline \multirow[b]{2}{*}{ Coals } & \multirow[b]{2}{*}{$\begin{array}{l}\text { Heat treatment } \\
\text { medium }\end{array}$} & \multirow[b]{2}{*}{$\begin{array}{c}\text { Extraction } \\
\text { solvent }\end{array}$} & \multicolumn{3}{|c|}{$\begin{array}{l}\text { Coal conversion rate }(\mathrm{wt} \%) \text { at various } \\
\text { heat treatment temperature, }{ }^{\circ} \mathrm{C}\end{array}$} \\
\hline & & & $202^{\circ} \mathrm{C}$ & $350^{\circ} \mathrm{C}$ & $400^{\circ} \mathrm{C}$ \\
\hline WVGS14321 & NMP & NMP & 41.6 & 43.4 & 70.0 \\
\hline PSOC3001 & NMP & NMP & 33.3 & 71.7 & $\begin{array}{c}81.7 \\
(117.6)^{*}\end{array}$ \\
\hline PSOC3001 & Creosote oil & NMP & & 66.1 & \\
\hline PSOC3001 & Coal-derived pitch & NMP & & 62.9 & \\
\hline WVSC001 & NMP & NMP & $\begin{array}{c}21.2 \\
(103.9)\end{array}$ & $\begin{array}{c}68.1 \\
(104.7)\end{array}$ & $\begin{array}{c}78.1 \\
(114.7)\end{array}$ \\
\hline
\end{tabular}

* The values in the parentheses are the recovery $w t \%(d b)$, i.e., $[($ residue + extract $)(g) / \operatorname{coal}(g)] \times 100 \%$

Figure 3.1 presents the temperature dependence of WVSC001 coal heat-treated at different temperatures using NMP as both heat treatment and extraction solvent. Below the $300^{\circ} \mathrm{C}$, the conversion yield increases slowly, but it increases greatly above $300^{\circ} \mathrm{C}$.

At temperatures between $250 \sim 300^{\circ} \mathrm{C}$, thermal decomposition of the coal begins to occur, such as the decomposition of carboxylic groups. This may weaken some coal molecular interactions (such as hydrogen bonds) formed by carboxylic group, and increase the coal swelling ratio as well as extraction yield [18]. However, the carboxylic group is not a main oxygen group in bituminous coal. Therefore, the decomposition of the carboxylic group may not be a main contribution to the conversion yield increase. Other factors must be involved, such as diffusion. The diffusion of solvent into the coal pores and extract moving out from the coal is always enhanced as the temperature increased.

Most coals start to decompose around 300 350C [19]. Coal conversion yield also increases significantly above $300^{\circ} \mathrm{C}$. The decomposition generates smaller molecules which go into 
solution; at the same time, in the absence of hydrogen radical, larger coal molecules could also be formed, and this part will be harder to dissolve. Since $70 \sim 80 \%$ coal is converted to NMP solubles, coal decomposition to smaller molecules appears to be the dominant reaction. In addition to the chemical decomposition, better solubility of solvent at high temperature and rearrangement of coal structure to a less aggregated state may also be important contributions to the high conversion yield.

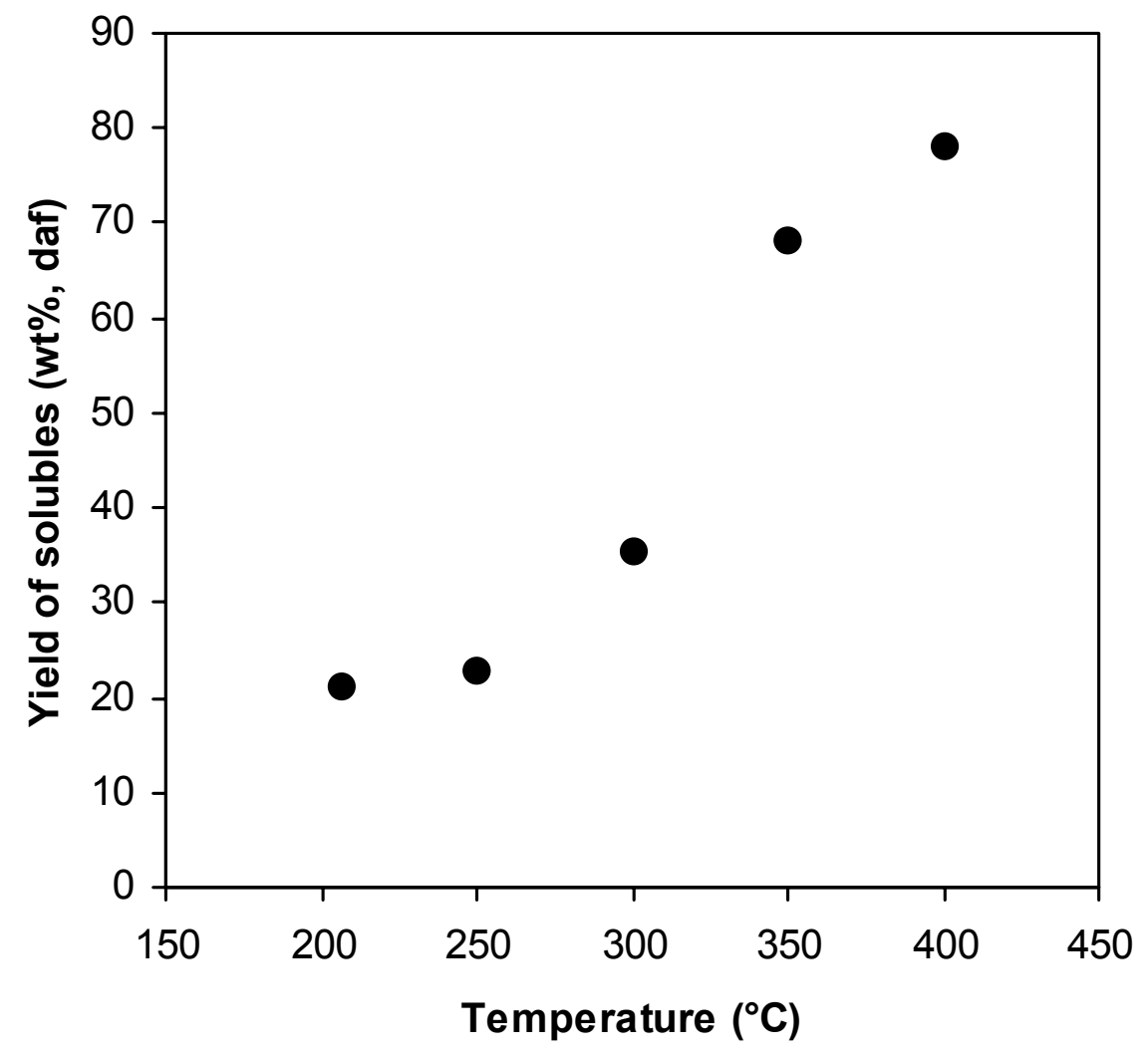

Figure 3.1 Change of coal conversion yield to NMP solubles as the heat treatment temperature increase. Heat treatment medium/extractant: NMP. Heat treatment/extraction carried out at same time for $1 \mathrm{hr}$. with agitation. Coal: WVCS001. 
The results in Table 3.13 show some stronger solvent retention, especially for the runs at $400^{\circ} \mathrm{C}$. Whether NMP reacts with coal at high temperature is still under investigation. However, the NMP retention can be diminished by means of water precipitation, i.e., the addition of excess water to the NMP extract solution to precipitate the extract. The FTIR spectra of the NMP coal extract obtained by vacuum evaporation of the NMP and water precipitation are compared in Figure 3. 2. The Carbonyl peak from NMP is greatly diminished in the extract precipitated by water. The mass balance is also decreased from $114.7 \%$ (using vacuum evaporation) to $102.7 \%$ (using water precipitation).

NMP retention will result in higher $\mathrm{N}$ and $\mathrm{O}$ content in the solubles as compared to the corresponding raw coal. The elemental analyses of raw coal and the solubles obtained at $400^{\circ} \mathrm{C}$ are listed in Table 3.14. For all the three coals, their NMP solubles have higher nitrogen content, which is due in part to the NMP retention. However, oxygen content in the solubles shows just a slight increase for PSOC3001 (increase from 6.10\% to 7.64\%), almost no increase for WVGS13421, and a decrease for WVSC0001(from 10.41\% to 7.25\%). This may be attributed to the loss of oxygen during the heat treatment at $400^{\circ} \mathrm{C}$. It is also observed that the higher oxygen content in the raw coal, the more oxygen is lost for its NMP solubles. The increased $\mathrm{H} / \mathrm{C}$ atomic ratio indicates the soluble fraction contains more light fractions, including solvent and the less aromatic species, etc.

Table 3.14 Comparison of the elemental composition of raw coal and the corresponding NMP solubles. [Thermal dissolution at $400^{\circ} \mathrm{C}$ using NMP as heat treatment medium and extraction solvent.]

\begin{tabular}{|l|c|c|c|c|c|c|}
\hline \multirow{2}{*}{ Samples } & \multicolumn{5}{|c|}{ Elemental composition, wt\%(daf) } & \multirow{2}{*}{ H/C } \\
\cline { 2 - 6 } & $\mathrm{C}$ & $\mathrm{H}$ & $\mathrm{N}$ & $\mathrm{S}$ & $\mathrm{O}$ & \\
by diff. & \\
\hline PSOC3001 raw coal & 86.69 & 4.92 & 1.52 & 0.766 & 6.10 & 0.681 \\
\hline $\begin{array}{l}\text { PSOC3001 } \\
400^{\circ} \mathrm{C} \text { NMP solubles }\end{array}$ & 80.99 & 5.61 & 4.68 & 0.973 & 7.75 & 0.830 \\
\hline WVGS13421 raw coal & 84.91 & 4.87 & 1.75 & 1.34 & 7.13 & 0.689 \\
\hline $\begin{array}{l}\text { WVGS13421, } \\
400^{\circ} \mathrm{C} \text { NMP soluble }\end{array}$ & 83.43 & 5.27 & 2.92 & 1.17 & 7.21 & 0.758 \\
\hline WVSC001 raw coal & 81.02 & 5.37 & 1.95 & 1.25 & 10.41 & 0.80 \\
\hline $\begin{array}{l}\text { WVGS001 } \\
400^{\circ} \mathrm{C} \text { NMP solubles }\end{array}$ & 82.73 & 5.85 & 2.89 & 1.28 & 7.25 & 0.85 \\
\hline
\end{tabular}




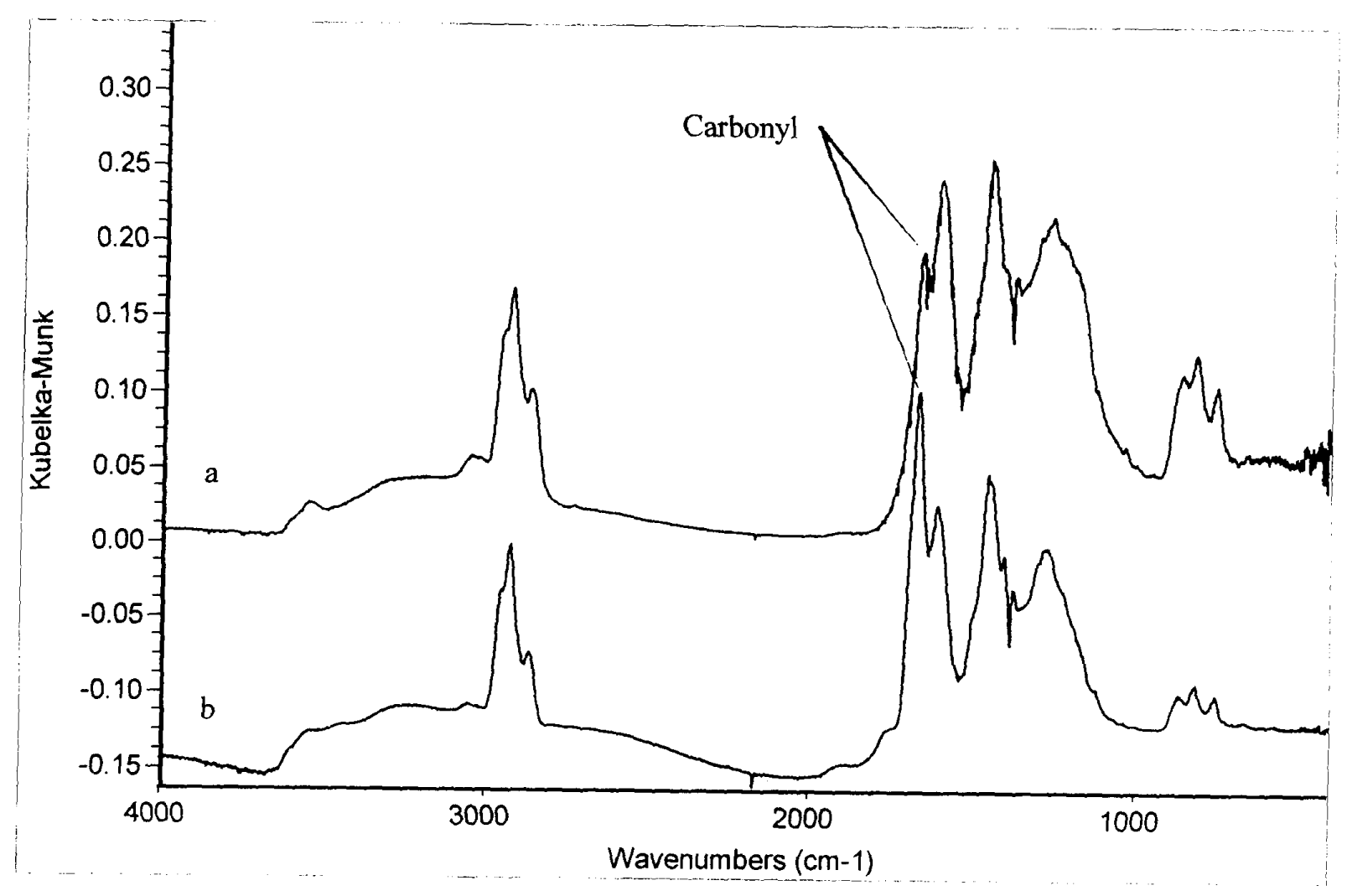

Figure 3. 2. FTIR spectra of NMP solubles from WVSC 001 coal heat treated at $400^{\circ} \mathrm{C}$ in NMP. a, solubles collected by water precipitation; $b$, solubles collected by vacuum evaporation.

\subsubsection{Kinetics of Coal Swelling in the Solvents}

The swelling of WVGS13421 and WVSC001 coal in NMP, DMF, DMAC and NMP/CS 2 mixed solvent was investigated. Their equilibrium swelling ratios at room temperature are indicated in Table 3.15.

Table 3.15 Swelling ratio of coal in solvents at room temperature

\begin{tabular}{|l|l|}
\hline Solvent & Swelling ratio, Q \\
&
\end{tabular}




\begin{tabular}{|l|c|c|}
\cline { 2 - 3 } \multicolumn{1}{l|}{} & WVGS-13421 & WVSC-001 \\
\hline DMF & 1.12 & 2.14 \\
\hline DMAC & 1.16 & 2.13 \\
\hline NMP & 1.24 & 2.25 \\
\hline NMP-CS & 1.65 & 2.46 \\
\hline
\end{tabular}

For the two coals, NMP gives higher swelling ratio than DMF and DMAC; NMP/CS 2 gives the highest swelling ratio. The results demonstrate that NMP and NMP/CS 2 are able to break more non-covalent interactions; therefore, they swell coal better. Swelling and extraction are strongly related, but are not simply correlated. Both coals have higher swelling ratios in $\mathrm{NMP}$ and NMP/CS . The swelling ratios for WVGS-13421 are much smaller than the corresponding swelling ratios for WVSC001. In order to obtain more insight into the swelling effects, WVSC001 coal was selected to investigate the swelling kinetics.

The swelling kinetics can be modeled by Equation 3.2 [20-21].

$$
\frac{M}{M^{*}}=\frac{Q-1}{Q^{*}-1}=k \times t^{n}
$$

where $\mathrm{M}=$ mass uptake of solvent by coal at a time $\mathrm{t}, \mathrm{g}$

$\mathrm{M}^{*}=$ equilibrium mass uptake of solvent by coal, $\mathrm{g}$

$\mathrm{k}=$ constant, whose units depend on the value of $\mathrm{n}$

$\mathrm{n}=$ exponent whose value indicates the nature of swelling process.

$\mathrm{t}=$ time, $\min$

$\mathrm{Q}=$ swelling ratio at time $\mathrm{t}$

$\mathrm{Q}^{*}=$ equilibrium swelling ratio

The value of $\mathrm{n}=0.43$ indicates Fickian diffusion of solvent while the value of $\mathrm{n}=0.85$ indicates case-II diffusion where the mechanism of diffusion of solvent is molecular relaxation of the coal network with the uptake of solvent. Equation 3. 2 is valid only for up to $60 \%$ uptake of the solvent relative to the equilibrium value [20-21].

The swelling curve and kinetics data are shown in Figures 3.3, 3.4 and 3.5. According to Equation 3.2, the plot of $\ln \left(\left(\mathrm{Q}^{-1)} /\left(\mathrm{Q}^{*}-1\right)\right)\right.$ vs. $\ln (\mathrm{t})$ is linear. From the data gathered here, 
it is clear that the diffusion of solvents DMF, DMAC and NMP follow the Fickian mechanism, since the value of $\mathrm{n}$ is close to 0.43 for all the three solvents.

It is observed that the higher the swelling ratio, the higher is the room temperature extraction yield. This is particularly observed when the swelling ratios and extraction yield of a coal are compared for NMP and NMP-CS 2 solvent system. However the relationship between them was not quantified at this time.

\subsection{Conclusions}

1. Of the various solvent tested herein; NMP is the best solvent for coal extraction. Its extraction yield for the selected bituminous coals ranges from 25 to $35 \%$ under reflux conditions.

2. When NMP, DMAC, DMF or cyclohexanone are mixed with $\mathrm{CS}_{2}(1: 1, \mathrm{v} / \mathrm{v})$, the mixed solvent shows much more extractability than the neat solvent.

3. Hydrogenation of coal greatly enhances its solubility in NMP, DMAC, DMF and cyclohexanone. Over $80 \mathrm{wt} \%$ hydrogenated coal can be dissolved by NMP even at room temperature.

4. Some additives are effective for the enhancement of the raw coal extraction yield. Tetrabutylammonium acetate and EDA increase the NMP extraction yield of WVGS 13421 coal from $35.9 \%$ to $50 \%$ and $60 \%$ respectively.

5. Thermal dissolution of raw coal at $400^{\circ} \mathrm{C}$ using NMP, creosote oil or coal-derived pitch as heat treatment media converts $70 \sim 80 \%$ of coal to NMP solubles.

6. The solvent swelling study indicates that the diffusion of NMP, DMAC and DMF in coal at room temperature is a Fickian process. 

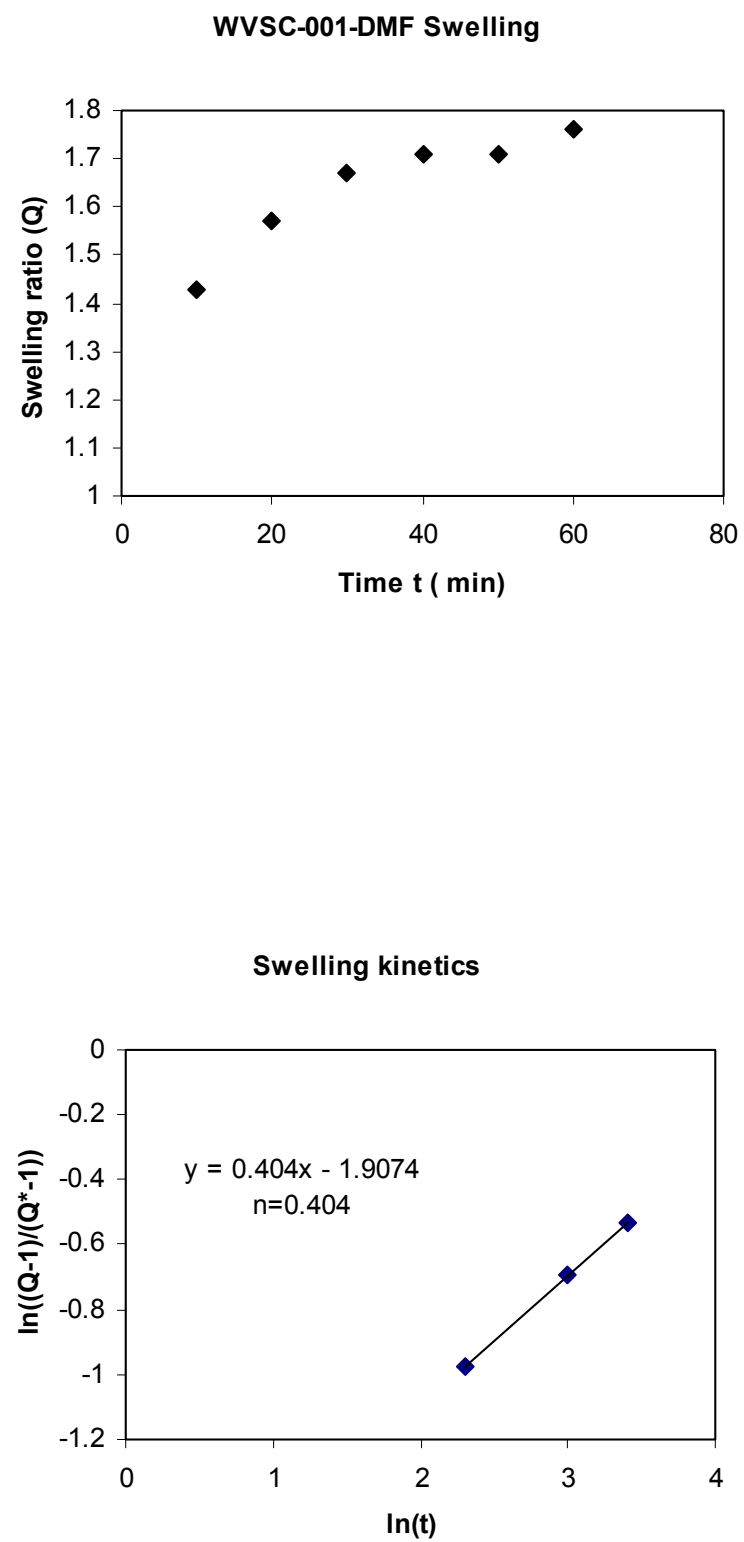

Figure 3.3 Swelling curve of WVSC-001 in DMF, top; and the kinetic curve from Equation 3.2, bottom. 

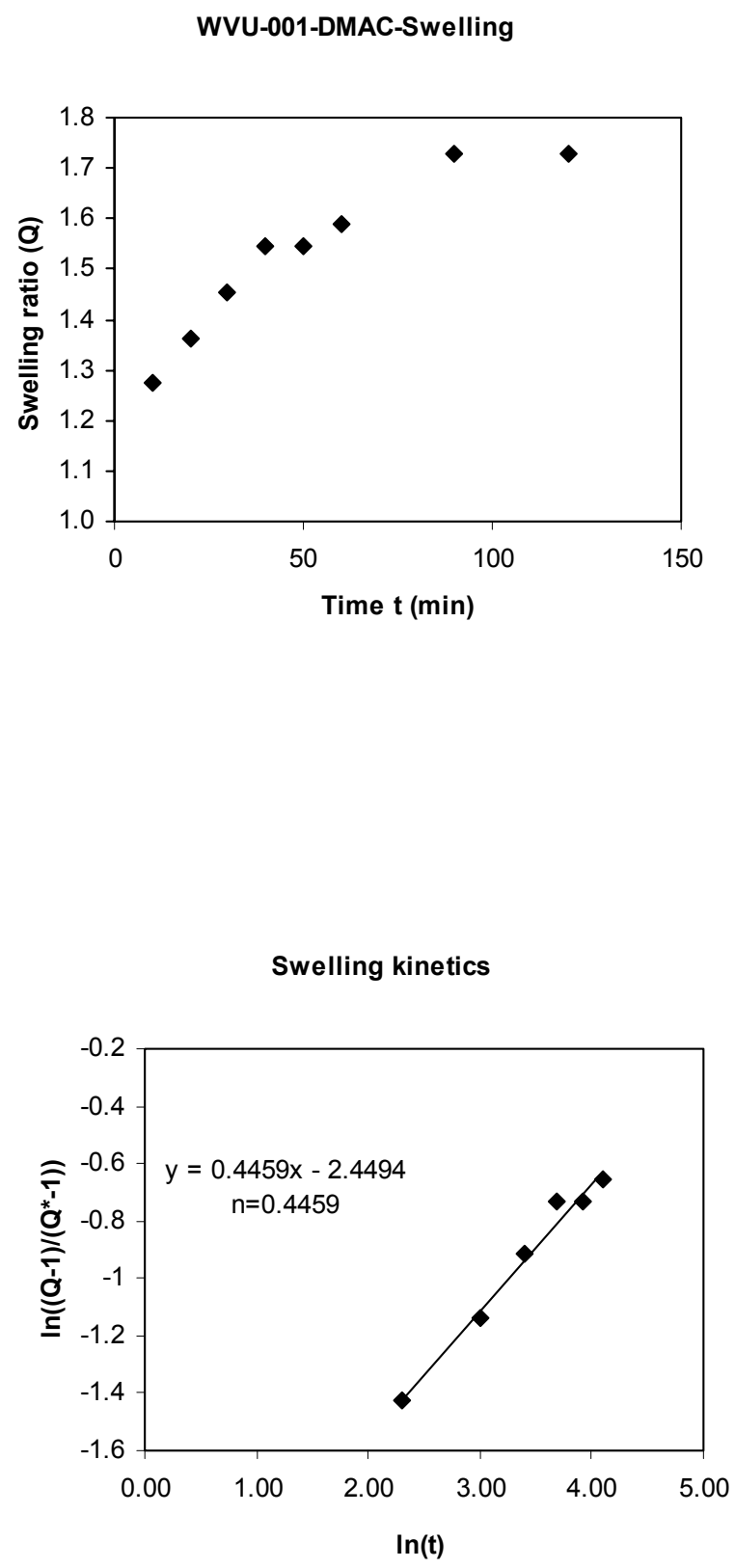

Figure 3.4 Swelling curve of WVSC-001 in DMAC, top; and the kinetic curve from Equation 3.2, bottom. 
WVSC-001-NMP Swelling

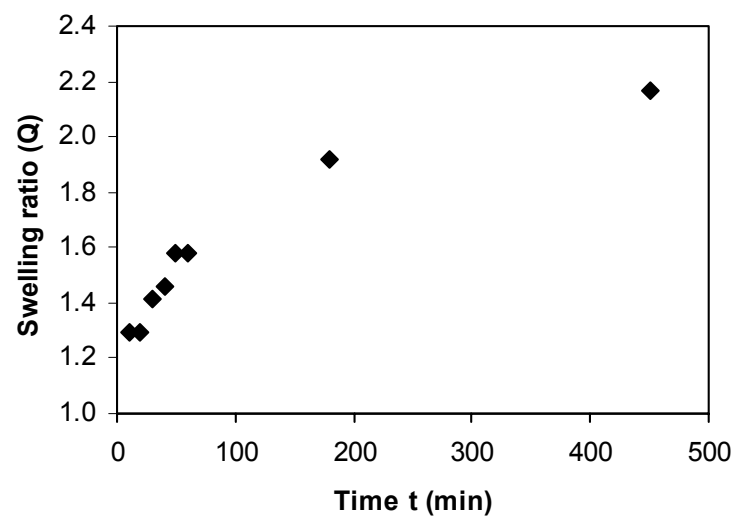

Swelling kinetics

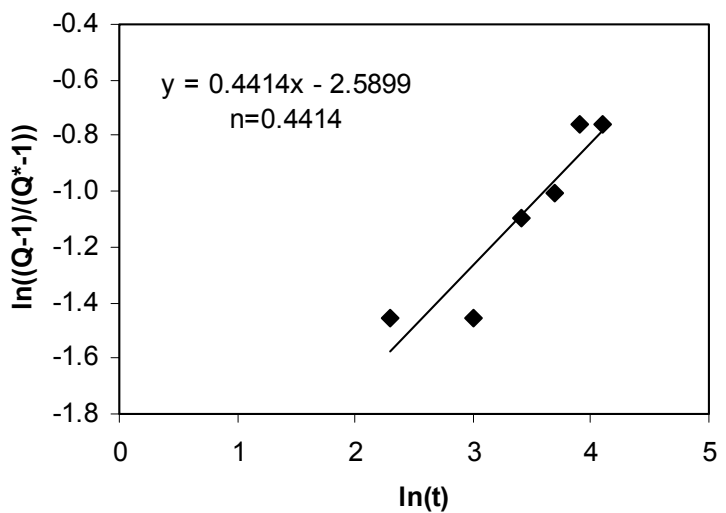

Figure 3.5 Swelling curve of WVSC-001 in NMP, top; and the kinetic curve from Equation 3.2, bottom. 


\subsection{References:}

1. Iino, M.; Takanihashi, T.; Ohsuga, H.; Toda, K. Fuel 1988, 67, 1639-1647

2. Dyrkacz, G. R. Energy Fuels Energy Fuels 2001, 15, 918-929

3. Dyrkacz, G. R.; Bloomquist, A. A. Energy Fuels 2001, 15, 1409-1413

4. Painter, P. C.; Sobkowiak, M.; Gamble, V. Prepr. Pap.-Am. Chem. Soc., Div. Fuel Chem. 1998, 43(4)(Aug 22-27), 913-916

5. Chen. C.; Iino, M. Fuel 2001, 80, 929-936

6. Coal based nuclear graphites for the new production gas cooled reactor, Final technical report, West Virginia University, Morgantown, WV., 1994

7. Matturro, M. G.; Liotta, R.; Reyholds, R. P. Energy Fuels 1990, 4(4), 346-51

8. Cooke, N. E.; Fuller, O. M.; Gaikwad, R. P. Fuel 1989, 68(10), 1227-33

9. Liu, H. T.; Ishizka, T.; Takanohashi, T.; Iino, M. Energy Fuels 1993, 7, 1108-1111

10. Chen, C.; Kurose, H.; Iino, M. Energy Fuels 1999, 13, 1180-1183

11. Giray, E. S. V.; Chen, C.; Takanohashi, T.; Iino, M. Fuel 2000, 79, 1533-1538

12. Chen, Iino, M. Prepr. Pap.-Am. Chem. Soc., Div. Fuel Chem. 2000, 45(4)(Aug 20-24), $865-869$

13. Van Bodegom, B. B.; van Veen, J. A. R.; van Kessel, G. M. M.; Sinnige-Nijssen, M. W. A.; Stuiver, H. C. M. Fuel 1984, 63, 364

14. Van Bodegom, B. B.; van Veen, J. A. R.; van Kessel, G. M. M.; Sinnige-Nijssen, M. W. A.; Stuiver, H. C. M. Fuel 1985, 64, 59

15. Pande, S.; Sharma, D. K. Energy Fuels 2002, 16, 194-204

16. Chen, C.; Iino, M. Energy Fuels 1999, 13, 1105-1106

17. Foster, R.; Horman, I. J.Chem. Soc. (B) 1966, 1049-52

18. Watanabe, I.; Sakanishi, K.; Mochida, I. Energy Fuels 2002, 16, 18-22 
19. van Krevelen, D. W. Coal; Elsevier: Netherlands, 1993.

20. Otake, Y.; Suuberg, E. M. Energy Fuels 1997, 11, 1155

21. Pande, S.; Sharma, D. K. Energy Fuels 2001, 15(5), 1063-68 